\title{
Guidelines for the Care and Welfare of Cephalopods in Research -A consensus based on an initiative by CephRes, FELASA and the Boyd Group
}

Laboratory Animals 2015, Vol. 49(S2) 1-90 (C) The Author(s) 2015 Reprints and permissions: sagepub.co.uk/ journalsPermissions.nav DOI: $10.1177 / 0023677215580006$ la.sagepub.com

(SAGE

\author{
Graziano Fiorito ${ }^{1,2}$, Andrea Affuso ${ }^{1,3}$, Jennifer Basil ${ }^{4}$, \\ Alison Cole ${ }^{2}$, Paolo de Girolamo, \\ Ludovic Dickel $^{7}$, Camino Gestal ${ }^{8}$, Frank Grasso ${ }^{9}$, Michael Kuba ${ }^{10}$, \\ Felix Mark ${ }^{11}$, Daniela Melillo ${ }^{1}$, Daniel Osorio ${ }^{12}$, Kerry Perkins ${ }^{12}$, \\ Giovanna Ponte ${ }^{2}$, Nadav Shashar ${ }^{13}$, David Smith ${ }^{14}$, Jane Smith ${ }^{15}$ \\ and Paul LR Andrews ${ }^{16,2}$
}

\begin{abstract}
This paper is the result of an international initiative and is a first attempt to develop guidelines for the care and welfare of cephalopods (i.e. nautilus, cuttlefish, squid and octopus) following the inclusion of this Class of $\sim 700$ known living invertebrate species in Directive 2010/63/EU. It aims to provide information for investigators, animal care committees, facility managers and animal care staff which will assist in improving both the care given to cephalopods, and the manner in which experimental procedures are carried out. Topics covered include: implications of the Directive for cephalopod research; project application requirements and the authorisation process; the application of the 3Rs principles; the need for harm-benefit assessment and severity classification. Guidelines and species-specific requirements are provided on: $i$. supply, capture and transport; ii. environmental characteristics and design of facilities le.g. water quality control, lighting requirements, vibration/noise sensitivity); iii. accommodation and care (including tank design), animal handling, feeding and environmental enrichment; $i v$. assessment of health and welfare le.g. monitoring biomarkers, physical and behavioural signs); $v$. approaches to severity assessment; vi. disease (causes, prevention and treatment); vii. scientific procedures, general anaesthesia and analgesia, methods of humane killing and confirmation of death. Sections covering risk assessment for operators and education and training requirements for carers, researchers and veterinarians are also included. Detailed aspects of care and welfare requirements for the main laboratory species currently used are summarised in Appendices. Knowledge gaps are highlighted to prompt research to enhance the evidence base for future revision of these guidelines.
\end{abstract}

\footnotetext{
${ }^{1}$ Stazione Zoologica Anton Dohrn, Villa Comunale, Napoli, Italy

${ }^{2}$ Association for Cephalopod Research 'CephRes', Italy

${ }^{3}$ Animal Model Facility-BIOGEM S.C.A.R.L., Ariano Irpino (AV), Italy

${ }^{4}$ Biology Department, Brooklyn College - CUNY Graduate Center, Brooklyn, NY, USA

${ }^{5}$ Department of Veterinary Medicine and Animal ProductionsUniversity of Naples Federico II, Napoli, Italy

${ }^{6}$ AISAL-Associazione Italiana per le Scienze degli Animali da Laboratorio, Milano, Italy

${ }^{7}$ Groupe mémoire et Plasticité comportementale, University of Caen Basse-Normandy, Caen, France

${ }^{8}$ Instituto de Investigaciones Marinas (IIM-CSIC), Vigo, Spain

${ }^{9}$ BioMimetic and Cognitive Robotics, Department of Psychology, Brooklyn College - CUNY, Brooklyn, NY, USA

${ }^{10}$ Max Planck Institute for Brain Research, Frankfurt, Germany
}

\footnotetext{
${ }^{11}$ Integrative Ecophysiology, Alfred Wegener Institute for Polar and Marine Research, Bremerhaven, Germany

${ }^{12}$ School of Life Sciences, University of Sussex, Sussex, UK

${ }^{13}$ Department of Life Sciences, Eilat Campus, Ben-Gurion University of the Negev, Beer, Sheva, Israel

${ }^{14}$ FELASA, Federation for Laboratory Animal Science Associations

${ }^{15}$ The Boyd Group, Hereford, UK

${ }^{16}$ Division of Biomedical Sciences, St George's University of London, London, UK

Corresponding author:

Graziano Fiorito, Research - CephRes, via dei Fiorentini 21, 80133 Napoli Italy.

Email: cephresacephalopodresearch.org
} 


\section{Keywords}

Cephalopods, Directive 2010/63/EU, animal welfare, 3Rs, invertebrates

\section{Contributors}

People listed here provided data, information and comments, and contributed to different extents during the preparation of this work.

The following list is arranged by country in alphabetical order; different contributors are merged by Institution.

\section{France}

Christelle Alves, Cecile Bellanger, Anne-Sophie Darmaillacq, Céline Gaudin

Groupe mémoire et Plasticité comportementale, EA4259, University of Caen Basse-Normandy, Caen, France

Joël Henry

Physiologie de la reproduction des Mollusques, University of Caen Basse-Normandy, Caen, France

\section{Germany}

Tamar Gutnick

Max Planck Institute for Brain Research, Frankfurt, Germany

\section{Italy}

Anna Di Cosmo

Department of Biology-University of Naples Federico II, Napoli, Italy

Carlo Di Cristo

Department of Biological and Environmental Sciences - University of Sannio, Benevento, Italy

Viola Galligioni

CIBio - Centre for Integrative Biology, Trento, Italy \& Association for Cephalopod Research 'CephRes', Italy

Anna Palumbo

Stazione Zoologica Anton Dohrn, Napoli, Italy

Perla Tedesco

Department of Biological and Environmental Science and Technologies-University of Salento, Lecce, Italy

Letizia Zullo

Istituto Italiano di Tecnologia, Department of Neuroscience and Brain Technologies, Genoa, Italy

\section{Portugal}

António Sykes

C. Mar - Centre of Marine Sciences, Universidade do Algarve, Faro, Portugal

\section{Spain}

Roger Villanueva López

Renewable Marine Resources Department-Institut de Ciències del Mar, Barcelona, Spain

\section{United Kingdom}

\section{Ngaire Dennison}

Home Office, Animals in Science Regulation Unit, Dundee, Scotland, UK

Penny Hawkins

RSPCA Research Animals Department, Southwater, West Sussex, UK

\section{United States of America}

Gregory J. Barord, Heike Neumeister, Janice Simmons, Roxanna Smallowitz

Biology Department, Brooklyn College-CUNY Graduate Center, Brooklyn, NY, USA

Jean Geary Boal

Biology Department, Millersville University, Millersville, PA, USA

Roger Hanlon, William Mebane Marine Resources Center, Marine Biological Laboratory, Woods Hole, MA, USA

Judit R Pungor Hopkins Marine Station of Stanford University, Pacific Grove, CA, USA

James B. Wood

Waikiki Aquarium, University of Hawaii-Manoa, Honolulu, HI, USA

\section{Introduction}

Cephalopods (i.e. nautilus, cuttlefish, squid and octopus) have been used for diverse scientific purposes across Europe for over 100 years. ${ }^{1,2}$ However, until recently, scientific procedures involving cephalopods have not been covered by EU regulations, with 
the exception of procedures using Octopus vulgaris in the United Kingdom (see discussion in Smith et al. ${ }^{3}$ ).

The inclusion of 'live cephalopods' (Article 1, 3b) in EU Directive 2010/63/EU on the 'protection of animals for scientific purposes' represents a landmark. It is the first time that an entire class of invertebrates, covering approximately 700 known species, ${ }^{4,5}$ has been included in laboratory animal legislation throughout the EU. The decision was largely based upon a review of the evidence for sentience and capacity to experience pain, suffering, distress and lasting harm (PSDLH) in cephalopods $^{6}$ (see also Table 1) which is now supported by more recent circumstantial (for reviews $\mathrm{see}^{7,8}$ ) and objective evidence $^{9-11}$ for the existence of nociceptors in cephalopods. Annexes III and IV to the EU Directive provide general guidance on care and accommodation requirements and methods of humane killing for all species covered by the Directive, but specific guidance is restricted to vertebrates, and there are no specific details for cephalopods.

Prompted by the need for guidelines on these and other matters covered by the Directive, members of the international cephalopod research community have met on several occasions over the past 3 years and have produced publications aimed at cephalopod researchers, on: $i$. requirements of the EU Directive, implementation, ethics and project review; ${ }^{3}$ ii. PSDLH, anaesthesia and humane killing; ${ }^{8}$ and iii. implications for neuroscience research and the Three Rs, i.e. Replacement, Reduction, Refinement. ${ }^{2}$

This work has led to the development of a set of consensus Guidelines for the Care and Welfare of Cephalopods in Research which aim to assist researchers in complying with the Directive, and are the subject of this paper. These guidelines have been developed as a joint initiative between CephRes (www.cephalopodresearch.org), FELASA (www.felasa.eu) and the Boyd Group UK (http://www.boyd-group.demon.co.uk/).

The Guidelines for the Care and Welfare of Cephalopods in Research, which should be regarded as a starting point for future developments, begin with a set of general principles of good practice, representing the present state of knowledge that may reasonably be applied to all cephalopods. These are followed by a tabulated set of specific guidelines (see Appendices) for typical cephalopod species, currently used in EU laboratories, which also reflect well-established principles.

\subsection{What is a cephalopod?}

For the purpose of these guidelines, cephalopods are defined as all living species that are members of the molluscan class Cephalopoda. ${ }^{4,5,12}$ The term 'live cephalopod' is not defined in the Directive, but guidance indicates that these animals are covered by the Directive from 'when they hatch'. 13,14

Cephalopods are characterised by bilateral body symmetry, a prominent head and a set of arms, including tentacles in Decapods, which are considered as muscular hydrostats and derived from the primitive molluscan foot. ${ }^{15-21}$ The class contains two, only distantly related, living subclasses: Nautiloidea (represented by Nautilus and Allonautilus) and Coleoidea, which includes cuttlefish, squid and octopuses. ${ }^{20,22}$ In the Nautiloidea, the external shell, common to the molluscan Bauplan, still exists, whereas in the Coleoidea it has been internalised or is absent. The variety of species that compose the taxon is reflected in the diversified habitats they have adapted to: oceans, benthic and pelagic zones, intertidal areas and deep sea, polar regions and the tropics. ${ }^{23-26}$

Understanding the requirements of a particular species in relation to its natural habitat is fundamental in maintaining healthy laboratory populations of cephalopods. Assumptions for housing, care and use of these animals based on fish, whilst appropriate in some circumstances, should be made with great caution as the evolutionary convergence between fish and cephalopods ${ }^{24,27}$ does not reflect the actual requirements of different species.

Generally, cephalopods have a high metabolic rate, grow rapidly and are short-lived. ${ }^{28,29}$ These animals are exothermic, highly adapted to the marine aquatic environment and are therefore unlikely to tolerate rapid or significant changes in the quality or temperature of the water they are housed in. They react rapidly to environmental changes/external stimuli with immediate physiological consequences that can be relatively long lasting. Such changes, as well as having potential welfare implications, will also impact upon experimental results.

Cephalopods are considered among the most 'advanced' invertebrates, having evolved many characteristic features such as relatively large, highly differentiated multi-lobular brains, a sophisticated set of sensory organs, fast jet-propelled locomotion, and complex and rich behavioural repertoires. ${ }^{25,30-37}$

\subsection{What the Directive 2010/63/EU means for cephalopod research}

The entry into force of the Directive 2010/63/EU (hereafter referred to as 'the Directive' $)^{38,39}$ means that, from 1st January 2013, scientific research and testing involving 'live cephalopods' is regulated by a legal framework at both EU and Member State levels, and as a consequence all scientific projects that cross the threshold set for regulation (i.e. involve procedures that may cause PSDLH equivalent to, or higher than that caused by the insertion of a hypodermic needle in line with good veterinary practice) will require authorisation by 


\begin{tabular}{|c|c|c|c|c|c|c|c|c|c|c|c|c|c|}
\hline & \multicolumn{2}{|c|}{ 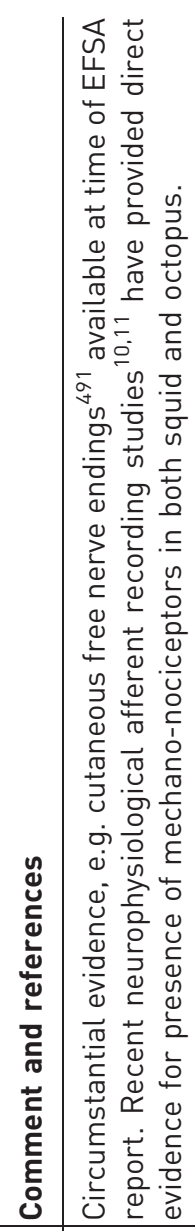 } & 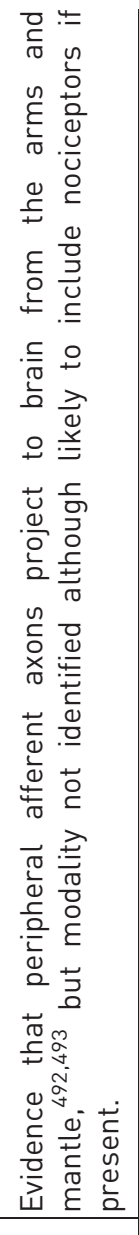 & 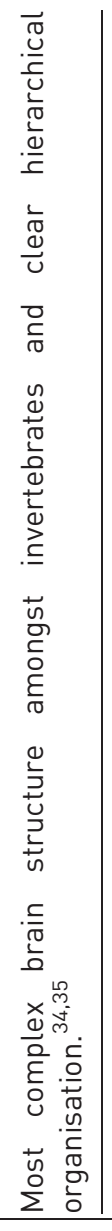 & 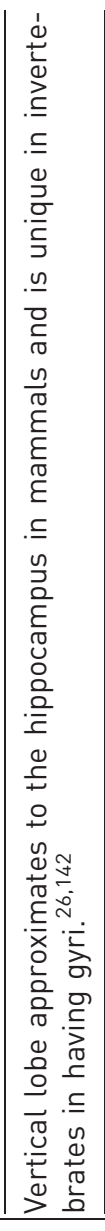 & 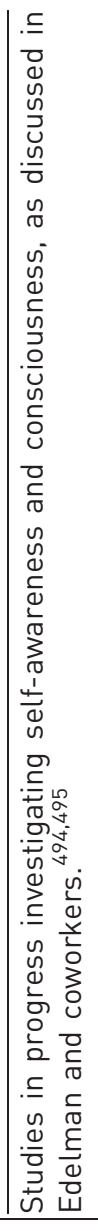 & 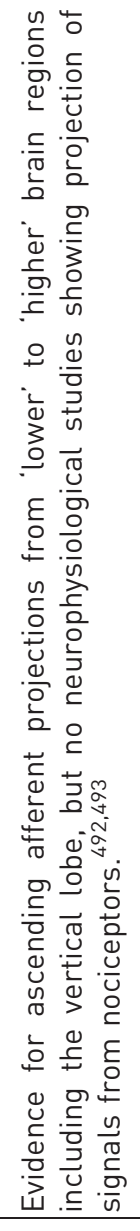 & 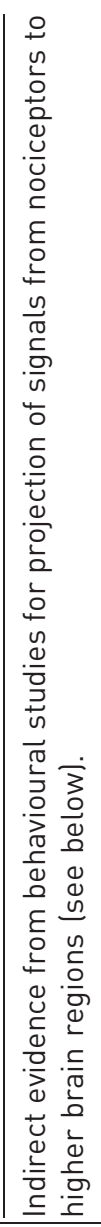 & 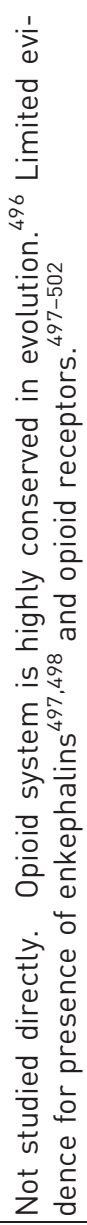 & 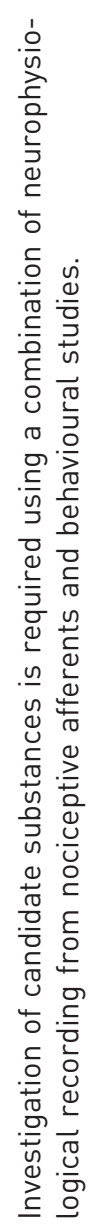 & 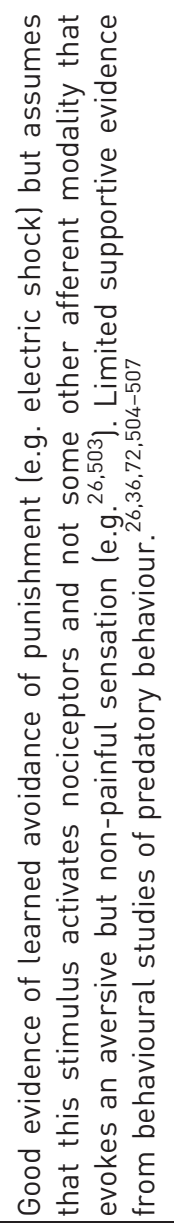 & \multicolumn{2}{|c|}{ 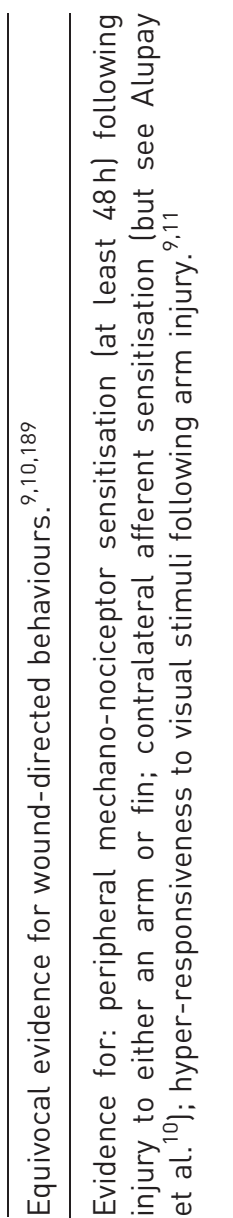 } \\
\hline & 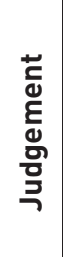 & $\underset{\varpi}{\sim}$ & 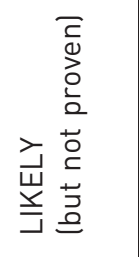 & $\underset{\varpi}{\breve{\varpi ~}}$ & & & 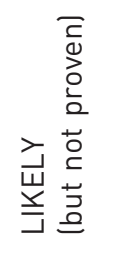 & & 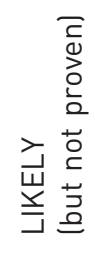 & 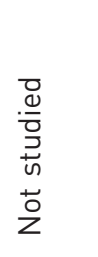 & 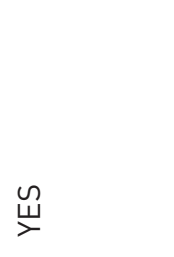 & & $\underset{\searrow}{\stackrel{\sim}{\Psi}}$ \\
\hline & 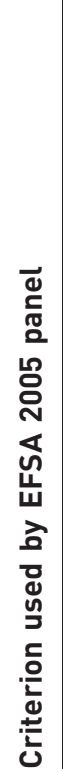 & 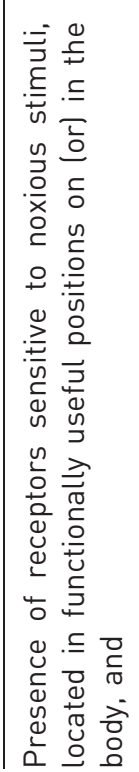 & 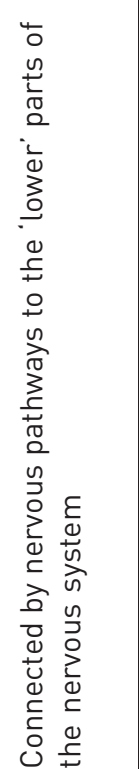 & 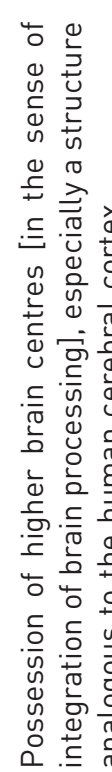 & & & 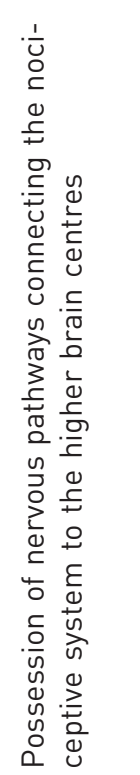 & & 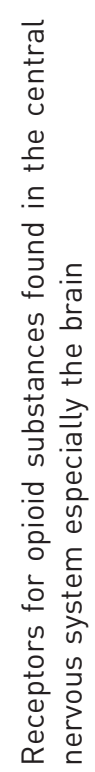 & 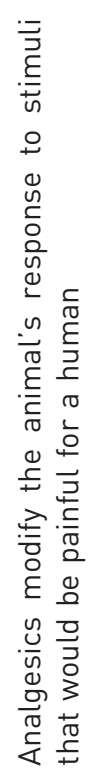 & 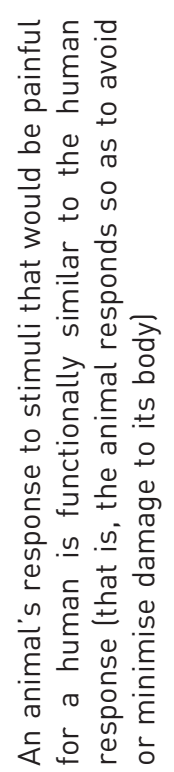 & & 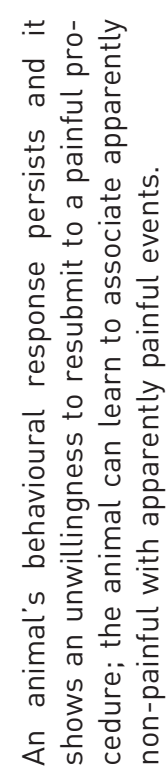 \\
\hline
\end{tabular}




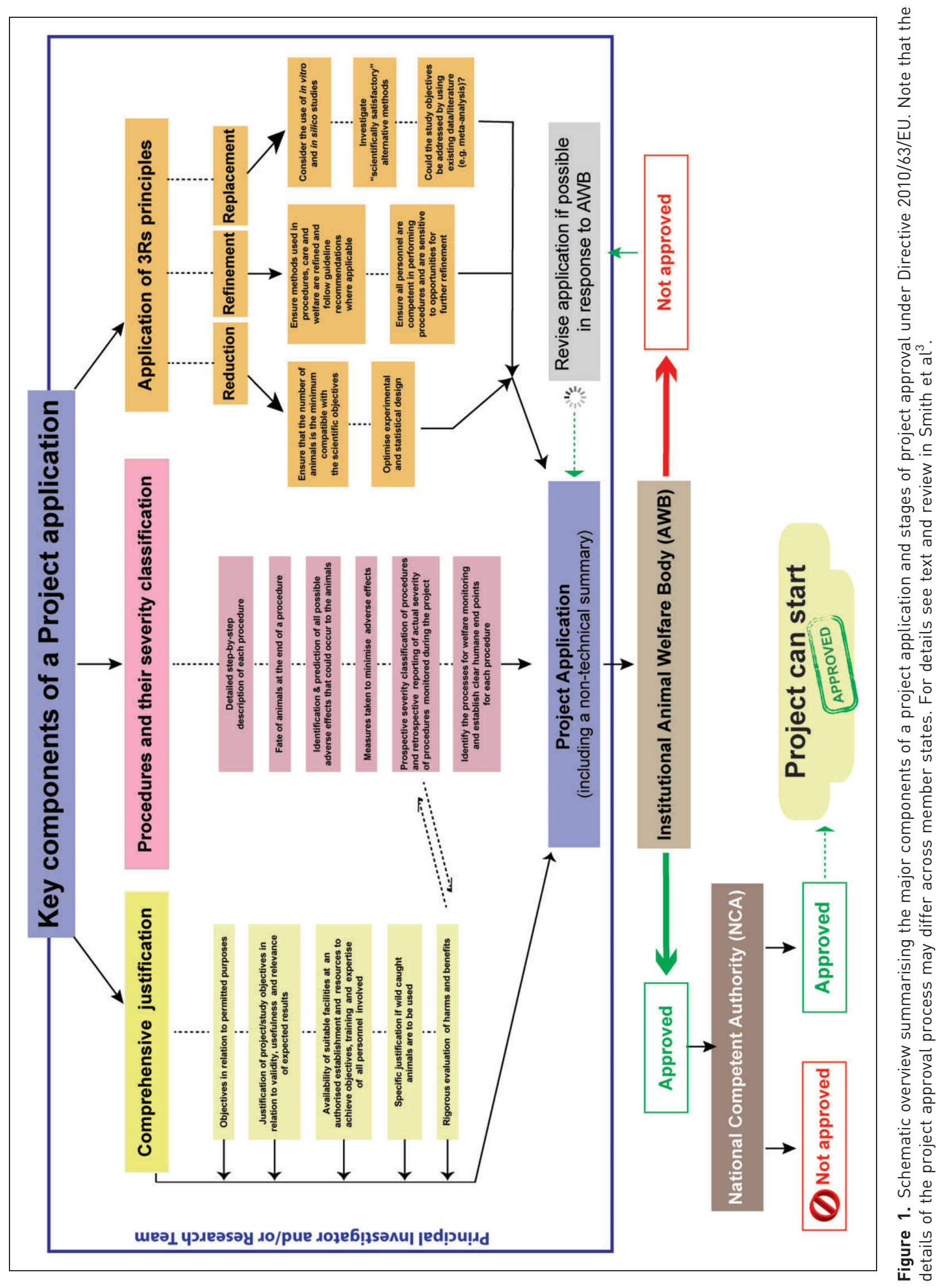


Text Box 1. Definition of key terms utilised in Directive 2010/63/EU.

A project is a programme of work with a defined scientific objective involving one or more procedures, which can run for a term of up to 5 years, after which authorisation must be renewed.

A procedure is any use of an animal covered by the Directive for experimental or other scientific or educational purposes, which 'may cause the animal pain, suffering, distress or lasting harm equivalent to or higher than that caused by the introduction of a needle in accordance with good veterinary practice'. This can include procedures that do not involve any 'invasive' technical acts such as administration of substances or surgery, but which cause psychological distress (such as anxiety) above the threshold level of suffering defined above. Unless specifically justified as part of the authorisation process, procedures may only be carried out at authorised user establishments.

Authorisation is limited to the procedures and purposes described in the application. If, during the life of the project, there is need for any amendments to the project plans that may have a negative impact on animal welfare, these must also be authorised.

A Competent Authority is a body responsible for implementing a specific task (or tasks), laid down by the Directive, within a Member State; for example, project evaluation and/or project authorisation. Member States must designate one or more competent authorities to fulfil these tasks.

Text Box 2. Definitions of the levels of severity of procedures according to Directive 2010/63/EU, Annex VIII; see also Lindl et al. ${ }^{490}$

Mild: Procedures on animals as a result of which the animals are likely to experience short-term mild pain, suffering or distress, as well as procedures with no significant impairment of the well-being or general condition of the animals shall be classified as 'mild'.

Moderate: Procedures on animals as a result of which the animals are likely to experience short-term moderate pain, suffering or distress, or long-lasting mild pain, suffering or distress as well as procedures that are likely to cause moderate impairment of the well-being or general condition of the animals shall be classified as 'moderate'.

Severe: Procedures on animals as a result of which the animals are likely to experience severe pain, suffering or distress, or long-lasting moderate pain, suffering or distress as well as procedures, that are likely to cause severe impairment of the well-being or general condition of the animals shall be classified as 'severe'. Note that the Competent Authority will require retrospective assessment of projects involving 'severe' procedures.

Non-recovery: Procedures which are performed entirely under general anaesthesia from which the animal shall not recover consciousness.

the National Competent Authority (see the list available at: http://ec.europa.eu/environment/chemicals/ lab_animals/ms_en.htm). ${ }^{3}$

Three key aspects of the project that will need to be considered by researchers and those responsible for animal care and welfare are outlined below (see also Figure 1). Specific topics for inclusion and consideration are listed in Appendix 1, and a more detailed overview of issues relating to implementation of the Directive for cephalopods can be found in Smith et al., ${ }^{3}$ which also includes some hypothetical worked examples of project review, particularly in relation to opportunities for implementing the 3 Rs (see 2.2.1 below).

\section{Project application requirements and authorisation process}

\subsection{Application requirements}

The key requirements of project authorisation are outlined here to provide a background to the technical sections, which aim to show how these requirements can be fulfilled specifically for cephalopods (Figure 1).

Before they can begin, all projects involving live cephalopods must be authorised by a competent authority appointed by the Member State in which the project is to take place (see Text Box 1 and Text Box 2 for definitions of key terms in the Directive). This will involve 'comprehensive project evaluation', 'taking into account ethical considerations' and 'implementation of principles of reduction, refinement and replacement' of the use of animals, the 3Rs (Recital 38 and 39).

An application for project authorisation must include, as a minimum, the project proposal and the items listed in Appendix 1. A non-technical project summary will be required unless waived by the National Competent Authority.

Applications must also include specific scientific justification for any requests for exemptions from certain requirements of the Directive (where permitted - see also Appendix 1). This will include requests for permission to: $i$. use an endangered cephalopod species 
where it falls within the criteria laid out in Article 7.1 of the Directive; $i i$. use cephalopods taken from the wild (Article 9, see also section 3.3 below); iii. carry out procedures in a place that is not an authorised users' establishment (Article 12); iv. re-use (in a different procedure) animals that have already undergone a procedure (Article 16, see also section 8.10 below); v. use drugs, such as neuromuscular blocking agents, that could stop or restrict an animal's ability to show pain, without an adequate level of anaesthesia or analgesia (Article 14\$3); vi. depart from any of the general standards of animal care and accommodation outlined in Section A of Annex III of the Directive (Article 33).

For species other than cephalopods, specific justification is also required for killing animals by a method not listed in Annex IV of the Directive, or for departing from species-specific standards of animal care and accommodation outlined in Section B of Directive Annex III. However, at present, cephalopods are not included in either of these Annexes.

Once a project is authorised and underway, it should continue to be critically evaluated by the Principal Investigator and all members of the project team, using the factors listed in Appendix 1, so as to ensure that ethical considerations and opportunities for implementing the $3 \mathrm{Rs}$ are identified and addressed in an ongoing process for the entire duration of the project, not only at the start.

\subsection{Factors to be evaluated in project authorisation and project operation}

The Directive sets out the factors that must be evaluated during project authorisation and throughout the lifetime of authorised projects. These factors are listed below as a series of action points and associated questions for consideration.

2.2.1 Implement the $3 R s$ to minimise the harms caused to the animals. The Three Rs (3Rs) principles were first described by Russell and Burch ${ }^{40}$ and are now internationally accepted as an essential requirement for the ethical and humane conduct of scientific studies involving animals (Recital 11; Articles 4 and 38 \$2b). In addition, it is widely recognised that implementation of the principles can enhance scientific quality. ${ }^{41-44,45}$

The following are examples of questions that will need to be addressed:

\section{Replacement}

- What on-going efforts will you make to identify 'scientifically satisfactory' alternative methods that could replace the use of some or all animals? (Article $4 \$ 1$ )
- Could you avoid the use of animals by asking different type of question, or making better use of existing data or literature to address the scientific objectives?

- Could in vitro studies or in silico-modelling be used to replace some or all of the animals?

\section{Reduction}

- How will you ensure that the number of animals used in the project, and in individual studies within the project, is 'reduced to a minimum without compromising the scientific objectives”? (Article 4\$2)

- Could any further reductions be made, e.g. by taking expert statistical advice to help optimise experimental and statistical design?

\section{Refinement}

- How have you refined the 'breeding, accommodation and care of the animals' and the 'methods used in procedures', so as to 'reduce to the minimum any possible pain, suffering, distress or lasting harm to the animals' throughout their lives? (Article 4\$3)

- Have you considered and implemented all the possibilities for refinement described elsewhere in these guidelines?

- How will you ensure that all relevant personnel working on the project are adequately educated and trained, and are supervised until they have demonstrated their competence in the procedures?

\subsubsection{Assess and assign the severity classification of} the procedures used in the project. Each procedure outlined in a project application must be classified according to the severity of its adverse effects on the animals (Article 38\$2c). This prospective severity classification sets an upper limit on the level of suffering that an individual animal undergoing the procedure is allowed to experience. ${ }^{46,47}$ The categories are: "nonrecovery' (for procedures carried out entirely under general anaesthesia from which the animal does not recover consciousness), 'mild', 'moderate' and 'severe' (Article $15 \$ 1$ and Annex VIII; see also section 8 below, and Text Box 2).

The following points must be considered for all projects, in order to fully address the 3 Rs and meet the requirements of the project evaluation process:

- Have you tried to identify and predict all possible adverse effects that could be caused to the animals in the project? Include any pain, distress, lasting harm and other forms of suffering, such as hunger, anxiety, boredom and osmotic or thermal stress caused to the animals, which may occur at any time during 
the animals' lifetime; for example, a result of capture and transport to the laboratory, routine handling, housing and husbandry, or method of killing, as well as the effects of the procedures themselves.

- Have you taken steps to minimise each of these adverse effects as far as possible, by: $i$. applying the 3 Rs in the design of procedures (see above), $i i$. using these guidelines, other relevant literature, advice from colleagues and from the institution's Animal Welfare Body to assist you?

- Based on the above, have you set prospective severity classifications (see section 8) and clear humane end-points for all procedures?

- How will you monitor the welfare of the animals used in the project, and when and how will you intervene to ensure that the animals do not suffer beyond the upper limit of severity needed to achieve the objectives of any particular procedure?

Member States must also collect retrospective information on the actual severity of procedures, after they have ended, and must make this publicly available on an annual basis (Article 54\$2). The assessment of actual severity is based on day-to-day observations of the animals, and the 'most severe' severity experienced by the animal is reported to the National Competent Authority. For example, if records of observations indicate that suffering is moderate at the beginning of a procedure and then mild for the remainder, actual severity for reporting purposes is 'moderate'.

Annex VIII of the Directive further explains the severity categories (see also section 8), and the European Commission has put together a series of examples* to illustrate the process of prospective severity classification, day-to-day observation and monitoring of animals and actual severity assessment.

2.2.3 Weigh the harms and benefits of the project and the individual studies within it. Taking into account all the points listed above:

- What is the basis for your overall assessment that 'the harm to the animals in terms of suffering, pain and distress is justified by the expected outcome, taking into account ethical considerations, and may ultimately benefit human beings, animals or the environment'? (Article 38\$2d)

- Have you explained this evaluation in your project application and, where relevant, your non-technical project summary (Article 43) - the latter using language suitable for the general public?

\footnotetext{
http://ec.europa.eu/environment/chemicals/lab_animals/pdf/
} examples.pdf
- How will you ensure that the "weighing of harms and benefits' is an on-going process throughout the project - i.e. part of the day-to-day practice of 'ethical science', and not just a one-off event at the time of authorisation?

A detailed examination of procedures for harm-benefit analysis in animal research and testing is available in a document from the Animal Procedures Committee in the UK. ${ }^{48}$

\section{Supply, capture and transport}

\subsection{Source of animals}

Article $9 \S 1$ of the Directive requires that animals must not be taken from the wild for use in procedures, unless an exemption has been granted by the relevant National Competent Authority, based on 'scientific justification to the effect that the purpose of the procedure cannot be achieved by the use of an animal that has been [purpose-] bred for use in procedures'.

This means that, in principle, cephalopods used for experimental or other scientific purposes should be bred and reared in captivity. However, there are significant difficulties in captive-breeding most cephalopod species (for exceptions, $\operatorname{se}^{2,49,50}$ ) and, therefore, this may not be feasible at the time of writing.

Development of more successful, standardised breeding procedures is urgently required. Article $38 \S 1 \mathrm{c}$ indicates that projects must be designed 'to enable procedures to be carried out in the most humane and environmentally sensitive manner possible'.

Where there is scientific justification for using animals taken from the wild, animals may be captured 'only by competent persons using methods which do not cause the animals avoidable pain, suffering, distress or lasting harm' (Article 9\$3). ${ }^{2}$ All those involved must observe a strict ethic of respectful treatment of animals, take into account their conservation status (section 3.2 below), and minimise the impact on the local ecosystem (section 3.3).

Care should be taken to prevent physical injury and stress to cephalopods at all stages in the supply chain, including capture (section 3.3), transportation (section 3.4), acclimatisation to laboratory conditions (3.5) and quarantine where required (3.6). It is also important to check local requirements for transport of animals in all countries along the route.

\subsection{Cephalopod species commonly used in research and conservation status}

An analysis of cephalopod species used in EU laboratories and the types of research undertaken can be found in Smith et al. ${ }^{3}$ Species from the main taxa of 
Table 2. Summary of major species of cephalopod used in research together with their source. Eggs may either wild caught or captive bred (e.g. for $S$. officinalis). See also reviews in Smith et al. $(2013)^{3}$ and Fiorito et al. (2014). ${ }^{2}$ The possibility of obtaining animals through a given source is indicated by ( $\mathrm{V}$, and $(\mathbf{X})$ indicates this source is considered possible, but not currently available. For details and comments on welfare issues see also text.

\begin{tabular}{llll}
\hline & Source & & \\
\cline { 2 - 4 } & Wild caught & Eggs & $\begin{array}{l}\text { Captive } \\
\text { bred animals }\end{array}$ \\
\hline Nautiloid & $V$ & & \\
Cuttlefish & $V$ & $V$ & X \\
Sepiolid & $V$ & X & X \\
Squid & $V$ & X & X \\
Octopus & $V$ & X & X \\
\hline
\end{tabular}

cephalopods used in research are shown in Table 2. Note that in some cases, e.g. Nautilus sp. or Idiosepius sp., animals are mainly available by importation and this requires permits and documentation from both exporting and importing countries ${ }^{\dagger}$.

At the time of writing, species of the Class Cephalopoda have not yet been assessed for possible inclusion in the IUCN Red List of Threatened Species $^{*}$ and hence none is listed as endangered. However, concerns are being raised for some rare species, based on local evidence and experience. Examples of locally protected species are: Euprymna scolopes in Hawaii; Octopus cyanea, Sepia elongata, Sepia pharaonis and Sepia prashadi in Israel (N. Shashar, pers. comm.); and some Mastigoteuthidae species in New Zealand. ${ }^{51}$ Indeed, assessments are now underway for cephalopods ${ }^{\S}$ under the Sampled Red List Index (SRLI) initiative**, which indicates the relative rate at which the conservation status of certain species groups changes over time, and aims to broaden the taxonomic coverage of the IUCN Red List.

\subsection{Capture methods}

Commonly used cephalopod species are listed in Table 2 together with information on currently available sources (e.g. wild, captive bred, eggs), and Table 3 indicates possible capture methods.

Several reviews describe commercial capture methods for cephalopod species. ${ }^{29,52}$ Current methods

\footnotetext{
†http://ec.europa.eu/environment/cites/info_permits_en.htm twww.iucnredlist.org

$\S_{\text {http://www.zsl.org/blogs/wild-science/animals-in-the-red }}$

${ }^{* *}$ http://www.zsl.org/science/indicators-and-assessments-unit/thesampled-red-list-index
}

include, but are not limited to, nets, traps and pots (see Table 3) ${ }^{53,54}$ Environmentally destructive methods (e.g. trawling) should be avoided wherever possible. Hand-jigging is considered the 'best' method for capturing squid, but may not be appropriate for all squid species. $^{51,55}$

Whichever method is used for capturing animals for research, it must not cause 'avoidable pain, suffering, distress or lasting harm' (Article 9). As noted above, animals may be captured only by competent persons. Moreover, researchers (and institutions) should only accept animals from suppliers who use appropriate capture and transportation methods; and the competence of third party providers should be evaluated based on the condition and survival of the animals supplied (see also section 3.4).

\subsection{Transport llocal, national and international)}

Transport of cephalopods should always be in welloxygenated seawater. ${ }^{56}$

Whenever possible and applicable to the research project, transport of eggs is the simplest, most successful, and, hence, preferable approach. Details of methods for egg transport are available for some cephalopod species. $^{56,57}$

When juvenile and adult cephalopods are transported, high survival rates should be achieved through careful selection of container type, maintenance of seawater quality in appropriate volumes and consideration of other measures to support animal welfare, such as food deprivation and cooling. The following discussion outlines general principles for transport of cephalopods, along with variations according to the duration of transport.

In the following sections, short-duration (i.e. short distance) transport is defined as requiring less than 2 hours; and long-duration (i.e. long distance) transport is for any longer period. These working definitions are based upon the consumption of available oxygen and detrimental changes in water chemistry (e.g. accumulation of ammonia and carbon dioxide and depletion of oxygen) as the duration of confinement for transport increases.

Since no specific systematic studies of transport methods for cephalopods are currently available, it is recommended that transport requirements should be based on the FAO guidelines for fish, ${ }^{58-60}$ paying particular attention to oxygen-sensitive species as they are considered to be comparable to cephalopods in their metabolic rate. Transport should also comply with the European Convention on the Protection of Animals during International Transport (ETS no 65, ETS no 193). 
Table 3. Schematic overview of the most common methods utilised for capture of cephalopod species for research purposes. See text for comments about welfare and environmental issues assocated with each method.

\begin{tabular}{|c|c|c|c|c|c|c|c|}
\hline & \multicolumn{7}{|l|}{ Capture methods } \\
\hline & Collection of eggs & Hand net & Traps & Pots & Hand jigging & SCUBA & Trawling \\
\hline Nautiloid & & & V & & & V & \\
\hline Cuttlefish & V & V & V & & & V & V \\
\hline Sepiolid & V & & & & & & \\
\hline Squid & V & V & V & & V & V & V \\
\hline Octopus & V & V & V & V & & V & V \\
\hline
\end{tabular}

3.4.1 Transport containers. Since seawater is crucial for the survival of the animals, steps must be taken to ensure that water will not leak at any time (e.g. by containing animals in double bags and placing bags within a sealed container). It should be noted that several cephalopod species (e.g. octopuses) are occasionally reported to cut and bite through thin plastic bags (M. Kuba, pers. comm.), hence stronger transport containers should be used for them. In any case, the external container should be able to contain the entire water volume even if all inside containers/ bags rupture.

Short-duration transport does not necessarily require animals to be placed into plastic bags, and other appropriate containers (large plastic bucket or box with a lid) may be preferred containing sufficient pre-oxygenated seawater to allow the animal to be completely immersed. Animals should be kept in dim or dark conditions, and movement and vibration of containers should be minimised.

For long-duration transport, similar to the protocol for transporting fishes ${ }^{58}$ and depending on body size, one animal should be placed with $1 / 3$ pre-oxygenated seawater and $2 / 3$ oxygen-enriched air in double common aquarium bags (see also description in $^{61-63}$ ). Once the bag is aerated, and the animal placed within it, it should be properly sealed (e.g. twisted at the top and folded over) and doubly secured (e.g. two rubber bands or cable grips). For transport periods over 12 hours, aeration and oxygenation may be necessary during transport, but care must be taken to use methods which do not add adverse conditions (i.e. bubbling) that may cause distress the animals.

Sealed holding bags (transparent to facilitate inspection if required) containing oxygenated seawater should be placed into insulated boxes (e.g. Styrofoam) to ensure that a temperature appropriate to the species is maintained during transport. The transport box should be darkened with a secure lid to keep the animals in darkness during transport, which reduces their stress.
Bags should be packed with cushioning material (e.g. paper, Styrofoam pellets) to ensure they do not move during transport.

The use of specialised aerating and insulated transport containers is not yet common for cephalopods. However, the development of specialised transport containers should be encouraged (for reviews on fish transport containers $\operatorname{see}^{58,60}$ ).

\subsubsection{Other factors for consideration. Food depriv-} ation before shipping may be desirable, where appropriate, dependent on $i$. shipping distance, $i i$. the species and iii. size of animal. It is common practice among cephalopod researchers to withdraw food before shipping. This is to help prevent fouling of the water and ammonia build-up during transport. The duration of food deprivation should be based upon a consideration of normal feeding frequency, oro-anal transit time and renal ammonium ion excretion. However, food deprivation may require authorisation by the National Competent Authority.

Food deprivation before transport, alongside lowered water temperature, can also help to avoid build-up of toxic ammonia and carbon dioxide. ${ }^{64,65}$

With the aim of lowering metabolic rate before shipping it has been suggested that pre-cooled seawater at $2-3^{\circ} \mathrm{C}$ above the thermal tolerance minimum of an individual species should be used, ensuring that the temperature stays at that level for the duration of transport (see e.g. for the giant octopus ${ }^{66}$ ). However, the impacts of this method on animal welfare are not yet known.

Sedation is not essential and is not recommended for transport of most cephalopods. However, sedation methods (i.e. cold water ${ }^{67}$ or 'chemical' methods ${ }^{68}$ ) has been used during transport in some instances with cephalopods.

It is interesting to note that in 1928 Grimpe suggested that very long duration transportation, i.e. requiring more than 2 days, should be achieved in 
steps allowing 'resting' periods in appropriate locations. ${ }^{56}$

\subsection{Acclimatisation after transport}

Transport inevitably causes animals stress. Therefore it is important to allow time for them to recover from transport-related effects, to acclimatise to the new conditions including possible differences in water quality, temperature, illumination, diet, and the shape and arrangement of the environment (i.e. the tank). Allowing time for acclimatisation is vital for both animal welfare and science, as stress can confound scientific results.

Almost all cephalopods are highly stenohaline and stenotherm, and care should be provided to avoid any difference in salinity and water temperature of the container utilised for the transport and the tank where the animals will be placed. In the case of significant difference in water temperature, an adjustment of the different 'media' after transport (e.g. placing the container inside the final tank for slow adaptation of the temperature to the final one) should be considered.

Cephalopods arriving in a facility should be examined for injuries or other health issues, and treated and/or quarantined (see section 3.6) or humanely killed (see section 8.11) where necessary. It is also recommended that $\mathrm{NH}_{4}$ and $\mathrm{CO}_{2}$ levels in the transport water are measured. Together, these observations can help in assessing the quality of transport methods and suppliers.

Based upon species-specific requirements (i.e. individual or group living; see also Appendix 2) animals should be placed in a holding tank until they are habituated to it. The requirements and duration of this practice are species-specific (generally from 1 to 5 days) and for experimental reasons may be reduced to a minimum (e.g. when studying individual preferences towards a prey item or a stimulus), because evidence is available for contextual learning to occur in most cephalopods. ${ }^{26,69-71}$

To facilitate the required 'habituation' to the captive situation, it is best if the holding tanks are designed according to the same principles as the maintenance/ experimental tanks for the species. Where food has been withdrawn during transport, a slow reintroduction is also recommended.

In the classic literature on cuttlefish and octopus, an adequate predatory performance is considered a sign of acclimatisation to a holding tank..$^{26,36,72}$ In addition, while excessive inking upon introduction to a new tank is a sign of stress, low swimming rates, reduced likelihood of inking in response to a small disturbance near a tank, can serve as indications of successful acclimatisation (N. Shashar, pers. comm.).

When moving animals from one tank to another within the laboratory a standardised, minimally stressful protocol should be applied. In these cases, only a brief acclimatisation time may be needed (in the order of minutes to few hours). However, the experimenter should be aware of potential handling and relocation stress and its physiological consequences that may impact on the research.

\subsection{Quarantine}

The purpose of quarantine after reception of animals is to isolate cephalopods from the main population accommodated in the facility to allow observation and testing until animals are assessed as healthy and free from potential infectious diseases. Individuals identified as ill should either be separated for treatment, if the cause can be identified and treatment is available (see section 7), or humanely killed (see section 8.11) and autopsied (see section 6.3).

Quarantine is also useful to isolate individuals that become sick while being maintained in the facility, allowing time for sanitary measures to be put in place and ensure appropriate containment of organisms and waters.

Currently, quarantine is not the general practice in the cephalopod research community. However, further studies are required based on recent research of cephalopod diseases and diffusion of parasites. ${ }^{73}$

The duration of quarantine should be sufficient to assure health of the individual animals. The needs of individual quarantined animals vary according to the biology and behaviour of the species (e.g. group holding maybe appropriate for gregarious species, but others may require individual accommodation; see also Appendix 2).

Quarantine should involve complete separation between animals to be quarantined and the current laboratory population; this should be achieved either by using separate rooms or equipping facilities with plastic screens to separate quarantine tanks from others. In addition, water supply should also be separate, to prevent diffusion of any potential harm to the water circulation and/or the environment. Similarly, equipment (e.g. nets) should not be shared between tanks.

During the quarantine period, animals should be monitored closely for unusual clinical signs or behaviours (see also sections 6 and 7), and detailed examinations (including autopsy; see section 6.3) made of any individuals who are considered to be 'abnormal'. In the cases of identification of diseased animals present in the laboratory holding facility, this should be regarded as a possible indicator of disease in the entire stock/holding group, and hence particular attention should be provided, and eventually they should all be treated or humanely killed. 


\section{Environment and its control}

\subsection{Seawater supply and quality}

Both natural and artificial seawater (see also below) are suitable for the maintenance of cephalopods. For fish, Annex III of Directive 2010/63/EU requires that 'an adequate water supply of suitable quality [is] provided at all times', and that 'at all times water quality parameters' are 'within the acceptable range that sustains normal activity and physiology for a given species and stage of development' and such requirements apply equally well to cephalopods.

4.1.1 Types of seawater circulation system. There are two principal seawater systems for keeping cephalopods: closed systems which recycle a reservoir of seawater, and open systems which either draw a continuous supply of water from the ocean (flow-through systems), or pump seawater into a reservoir and regularly replenish it with fresh seawater (semi-closed systems).

Closed systems have the advantage of enabling control of all parameters of the environment, but are more costly due to the need for additional environmental monitoring and control equipment.

Open systems rely on fresh seawater being drawn from the ocean. While this has some advantages (especially not needing expensive filters), it limits the facility to keeping animals that can live within the given water parameters. For example, non-native species cannot be kept in this type of system without considerable efforts purifying and sterilising the reflow. Naturally open systems are also limited to areas close to the shore.

Closed systems: Efficient and relatively easy-to-maintain closed aquarium systems have been developed for cephalopods. ${ }^{74-78}$ Commercially available artificial seawater preparations are considered adequate, provided they contain the necessary substances and trace elements to meet the physiological needs of the particular cephalopod species, and for this reason mixtures designed for marine invertebrates and corals should be preferred.

Water flow in recirculating systems or filtration within enclosures should be sufficient to remove suspended waste and to ensure that water quality parameters are maintained within acceptable levels. Filtering systems in recycling/recirculating seawater should be adequately planned and maintained. ${ }^{79,80}$ Appropriate processes for monitoring water parameters should be implemented, and alarms in place to ensure flow and seawater levels are adequate.

Open systems: seawater drawn from the ocean should be tested for contaminants and pathogens, and treated to remove them. The water supply should also be evaluated to ensure that there is sufficient capacity, including ability to cope with periods of maximum demand and emergency situations. To protect animals from potential contaminants, other measures, such as appropriate filtering or a reverse osmosis system, may be required. ${ }^{79,80}$

Tests to determine the chemical composition and presence of contaminants/toxins will determine the treatment necessary to make the water suitable for use. Seasonal factors such as phyto- or zoo-plankton blooms, tidal cycles, and seasonal seawater mass turnovers can have periodic effects (on a scale of hours, days or months) for seawater and these should be anticipated.

All systems: water flow should enable cephalopods to maintain normal locomotion and behaviour. Cephalopods can use rapid expulsion of water through the funnel to power jet propulsion, which results in swift movement. Cuttlefish and squid have fin-like structures on the mantle to assist in locomotion. Squid are in continuous motion due to their pelagic nature, thus water flow ${ }^{75,77}$ needs to be sufficient to ensure appropriate life-style requirements, and adequate water quality including quick removal of ink (if released).

4.1.2 Water quality. As for other aquatic species, water quality is the most important factor in maintaining the health and well-being of cephalopods. Insufficient water quality will cause stress and disease. Water-quality parameters should at all times be within the acceptable range that sustains normal activity and physiology for a given species and individual (see Appendix 2); and should remain stable, unless the life style of a given species requires changes (e.g. because of large vertical migrations during a day or seasonal changes). Optimum conditions vary between species (e.g. deepsea benthic octopuses are especially sensitive to changes), between life-stages (e.g. paralarvae, juveniles, and adults) and according to physiological status of the individual (e.g. females preparing to lay eggs).

Most cephalopods show little adaptability to changing water-quality conditions, and so when animals need to be moved between tanks or systems, it is important to ensure that water parameters are mirrored and maintained. If this is not possible, gradual acclimatisation will be needed (see section 3.5), as for other marine invertebrates and fishes.

Dissolved oxygen, $\mathrm{pH}$, carbon dioxide, nitrogenous compounds and salinity should be monitored and maintained according to the appropriate range for each species. Appendix 2 provides a list of water quality criteria for optimum health and welfare of cephalopods; for more detailed discussion of monitoring water quality see below.

\footnotetext{
"but this may be difficult to achieve in laboratory facilities.
} 


\subsection{Monitoring water quality $\mathrm{IO}_{2}, \mathrm{pH}, \mathrm{CO}_{2}$, nitrogenous material, salinity and metals)}

Seawater parameters should be monitored (continuously by specific electrodes or intermittently by chemical methods) and recorded at an appropriate frequency (at least daily), thus allowing proactive, rather than reactive, management of water quality. Parameters that need to be measured and the frequency of measurement vary (see also Appendix 2), depending on whether the system is open or closed. For example, while there may be no need to measure nitrites/nitrate in a high volume flow-through system (depending on the source of the water), such measurements are critical with recirculation systems.

At a minimum, environmental monitoring systems should provide information on water flow, oxygen saturation and water temperature. Parameters measured should also be relevant to the health and welfare of the particular species housed in the facility (see Appendix 2). In general, recirculation systems should be monitored for a larger number of parameters, including, but not restricted to, dissolved oxygen, $\mathrm{pH}$, nitrogenous material, salinity, total dissolved salts and temperature (see below). As a minimum, water quality analysis should be carried out at times of greatest demand on the system (usually after feeding) to identify potential problems.

Water and tanks should be kept clean particularly of faeces and uneaten food. In semi-open and closed systems, water should be treated to reduce potential pathologies, for example, using UV light or ozone. If ozone is used, measurements of ozone concentrations and/or redox potential of the reflow entering the system are necessary to avoid toxicity.

Alarm and notification values must be set and their significance as potential indicators of problems in the system explained to all relevant personnel. There must be an agreed, clear protocol for contacting those responsible for the facility when problems are identified outside of normal working hours.

The monitored parameters should be recorded and the information stored for at least 5 years. For all parameters considered below and for techniques of keeping animals information is also available through a recent compilation of research on the culture of cephalopods. ${ }^{50}$

4.2.1 Oxygen. Cephalopods have high metabolic rates, so oxygen concentration should generally be kept high (close to saturation); and where tank inflow is not sufficient, supplementary aeration of the water must be provided. However, supersaturating the water is not advisable as it may cause gas bubbles to become trapped in the mucus layer of the animals, thus limiting gas exchange at the gills (for octopus: G. Fiorito, pers. comm.; for several cephalopod species: J. Rundle, pers. comm.).

Information on oxygen consumption in some cephalopods is provided by Winterstein ${ }^{81}$ (see also $^{82,83,83-87}$ ) and reccomended requirements are summarised in Appendix 2.

It is important to note that monitoring oxygen levels is not informative of the oxygen available to the cephalopod unless it is combined with measurements of $\mathrm{pH}$ (see next section).

4.2.2 $\mathrm{pH}$. Due to the effects of $\mathrm{pH}$ on the carriage of oxygen by blood pigments, cephalopods tolerate low $\mathrm{pH}$ poorly. ${ }^{88-96}$ Regular measurement and careful maintenance of $\mathrm{pH}$ is therefore critical. Acceptable $\mathrm{pH}$ levels depend on many water quality factors, for example, carbon dioxide (see below), and calcium; as a consequence, control of soluble gases and water solutes is important. This may be a particular issue for establishments that use synthetic marine salts rather than natural seawater and closed filtration systems, especially where water changes are limited. Acceptable $\mathrm{pH}$ values for keeping cephalopods are summarised in Appendix 2.

4.2.3 Carbon dioxide. Carbon dioxide is produced during respiration and dissolves in water to form carbonic acid, thus lowering the $\mathrm{pH}$. Since stability of $\mathrm{pH}$ is very important, accumulation of carbon dioxide should be avoided. Situations that may increase carbon dioxide levels include high stocking density and poor aeration.

Care should be taken that water supply systems, particularly in the case of groundwater-based systems, do not introduce harmful quantities of carbon dioxide to the enclosures.

4.2.4 Nitrogenous material. As cephalopods are carnivores, hence requiring a high protein diet, the accumulation of potentially toxic nitrogenous compounds can be a problem, particularly in closed systems. It is also necessary to avoid accumulations of ink (especially when keeping cuttlefish, that ink in large volumes). Timely removal of uncomsumed food, use of adequate protein skimmers and suitable water flow rates, along with careful water filtering in closed systems, will help to reduce levels of organic waste, including ammonia, nitrites and nitrates. It is also beneficial if the flow rate in the tanks can be adjusted to different situations (e.g. increased after inking). Additional water changes can provide a supplementary means of removal of waste products and substances such as ink.

Build up of nitrogenous compounds may lead to behavioural changes and/or changes in skin 
colouration in cephalopods. For example, at nitrate levels $>80 \mathrm{mg} / 1$ cuttlefish become very agitated, will ink profusely and their skin tone may be dark; and larvae and hatchlings are more vulnerable to bacterial disease (A. Sykes, pers. comm.).

Levels of nitrogen compounds tolerable by different species are reviewed in Appendix 2 (see also Iglesias et al. ${ }^{50}$ ).

4.2.5 Salinity and metals in seawater. As cephalopods are marine organisms, maintaining an appropriate salt concentration is vital. The salinity should match the natural habitat of the animals.

Commercially available artificial seawater preparations and especially any mixture designed for marine invertebrates and corals are considered adequate as they contain all the necessary substances and trace elements to keep cephalopods in good health. However, in accordance with instructions for the different brands of salt, some trace elements, in particular strontium and calcium should be monitored and added if necessary.

Copper and its alloys are considered to affect the salinity and 'poison' the seawater ${ }^{56}$ and therefore should be avoided in any system holding cephalopods.

Cephalopods are reported to accumulate and be sensitive to heavy metals so care should be taken to ensure these are monitored and maintained within normal ambient ranges for the species, to limit potential damage (see above and also $^{2}$ for relevant literature).

\subsection{Lighting control}

Light influences, either directly or indirectly, almost all physiological and behavioural processes in cephalopods, including growth, development and reproduction. Lighting requirements vary between cephalopod species, and both wavelengths and intensity of lighting should 'satisfy the biological requirements of the animals', where these are known (Directive, Annex III, section 2.2a). The natural history of the species, in particular the normal living depth, can provide clues to help meet the species lightling preferences: for example there are many cephalopods that prefer very little light. There is limited specific knowledge on wavelength perception for almost all cephalopod species. ${ }^{97}$ However, it is estimated that simulated sun-light equivalent to that normally experienced at 3-8 $\mathrm{m}$ depth at sea should be acceptable for the majority of cephalopod species commonly used as laboratory animals (but see further information in Appendix 2).

Photoperiod should also be maintained according to the natural requirements of the species. ${ }^{98-103}$ However, there is evidence that some cephalopods may easily adapt to changes in day/night regime. ${ }^{26,104-106}$
Where task lighting is needed for people working in the room, it should be restricted in its dispersion, and/ or be placed below the level of the tank surface, to reduce disturbance to the animals. Use of automated dimmer controls that allow light intensity to be gradually increased is important and recommended (G. Fiorito, pers. comm.; see also ${ }^{107}$ ). For example, for decapods and nocturnal octopuses sudden changes in light level may cause escape reactions, and in some cases inking, thus a simulated dusk and dawn period is desirable. Care should also be taken to ensure that animals are not disturbed by night-time security lighting entering through windows in the holding facilities. The output of fluorescent lights can be diminished by using dummy bulbs to reduce light levels.

\subsection{Temperature control}

Water temperature should be controlled within the natural range for the species; and, where necessary, appropriate chilling/heating equipment must be used to ensure the optimal temperature range for the animals.

Cephalopod species vary in their sensitivity to changes in water temperature. In general, higher water temperatures create problems for animals from temperate climates like octopus and the cuttlefish. Transitions of temperature should not be sudden. ${ }^{56,108}$ Where water changes are performed on larger scales, temperature spikes, which may cause adverse effects, should be controlled and avoided.

\subsection{Noise and vibration control}

Background noise, and vibration from housing systems, such as pumps or ventilation units, should be minimised as they are likely to impact on cephalopod welfare.

Several studies suggest that cephalopods can detect sounds even at low frequencies, ${ }^{109-115}$ and other recent work shows that cephalopods are as likely as other marine organisms, to suffer from low-frequency noise traumas. ${ }^{116-118}$

In common with other aquatic species, cephalopods dislike vibrations, such as drilling or banging on tank sides, and some species, such as cuttlefish, may respond by inking. Therefore, the most important aspect of sound reduction is to minimise disruption and avoid sudden noises, which could startle the animals.

\subsection{Aquatic life support systems and emergencies}

Tanks should be built so that complete drainage is impossible when they are inhabited (although ability to drain tanks may be required for cleaning purposes). 
Two independent sources of water movement/ oxygen supply are also recommended, for example, pumps for water circulation plus extra air sources to provide additional aeration.

Electronic alarm systems help to ensure that problems in a system are detected promptly. All facilities must have an emergency plan in place should problems arise (including out-of-hours), with clear actions that are understood by all and effectively communicated to everyone. There must be a backup system to enable an appropriate response to the worst case scenario of a complete system failure, and so avoid circumstances in which animals would have to be humanely killed due to suffering from anoxia or a build up of organic waste.

\section{Accommodation and care}

\subsection{Background and requirements of the Directive}

About 50 species of cephalopod have been kept successfully in aquaria (M. Kuba, pers. comm.; see also ${ }^{108}$ ). These range from small species such as bobtail squid (E. scolopes) to larger pelagic squid (e.g. Loligo vulgaris, Doryteuthis pealeii), octopuses (e.g. O. vulgaris, Eledone cirrhosa) and cuttlefishes (Sepia officinalis), and the giant pacific octopus (Enteroctopus dofleini).

At the Stazione Zoologica di Napoli, considered to be the first large-scale facility for the maintenance of cephalopods $^{56}$ (mostly for cuttlefishes and octopuses, A. Droesher and G. Fiorito, pers. comm.), outdoor tanks were preferred to indoor rooms, to allow animals to be kept in natural light with seasonal daylength. However, shading was provided to reduce direct sunlight to the animals. In the following years, indoor tanks were installed and artificial lighting was introduced to supplement natural illumination (A. Droesher and G. Fiorito, pers. comm.).

The knowledge accumulated in various laboratories around the world, with a variety of cephalopod species, supplemented the original studies at Stazione Zoologica and facilitated the design of closed systems for maintenance of species which adapt less readily to laboratory housing such as squid (for review and methods see $^{75}$ ).

Annex III of Directive 2010/63/EU sets out requirements for care and accommodation of animals. Section A lists general requirements pertaining to all species and section B lists species-specific requirements, for all vertebrate classes, including brief guidance for fish (but with no distinction between the different classes of fish). Some of these Section B requirements for fish might also apply to cephalopods, ${ }^{2}$ but cephalopods are not specifically mentioned in Annex III.

\subsection{Holding facilities for cephalopods}

Planning design and maintenance of new accommodation facilities for cephalopods should take into consideration key points outlined by the Committee for the Update of the Guide for the Care and Use of Laboratory Animals. ${ }^{119}$

Access to the facility should be allowed only to people who have received relevant training and have a legitimate need for access. Movements of personnel inside the facility should also be monitored and controlled to minimise disturbance to the animals and ensure biosecurity, which may require measures such as physical barriers and access restriction/control.

Walls of holding rooms should generally be of dark neutral and continuous colours. However, very dark colours may make it difficult to identify dirty areas, so specific evaluation of the appropriate colour may be required.

Cephalopods require large volumes of seawater. All facilities should have an emergency contingency capacity, capable of maintaining aerated and filtered seawater should normal systems fail. Monitoring systems including remote alarm notification should be designed and used in cephalopod facilities.

Noise should be minimised to avoid disturbing animals in both housing and experimental rooms. When applying sound-attenuating material to the ceiling or walls, always consider that it has to be sanitisable. All vibration sources (e.g. mechanical equipment, electrical switches, through ground-borne transmission) should be identified and vibration isolation methods should be used to reduce noise (e.g. by placing equipment on rubber pads). Noise-producing support functions, such as tank and filter washing, should be separated from housing and experimental areas, wherever possible.

Fire and environmental-monitoring alarm systems should be selected and positioned to minimise potential disturbance to animals.

All procedures and other manipulations of living animals should be carried out inside the facility to minimise stress to the animals, unless there is scientific justification for doing otherwsise. Therefore, a typical cephalopod facility should have available: $i$. adult animal housing/ holding room(s) divided by species if possible, and breeding/'hatching' $\operatorname{room}(\mathrm{s})$; ii. quarantine $\operatorname{room}(\mathrm{s})$ (if needed); iii. an area for acclimatisation of animals; $i v$. procedure rooms separated from holding and breeding rooms, for experimental techniques, including regulated procedures (e.g. surgery, behavioural experiments, imaging, clinical treatment, humane killing, necropsy, etc.); $v$. separate 'service' rooms for storage of food, supplies, chemicals, etc., and for waste (including biological material) storage before incineration or removal.

Shared facilities, where cephalopods are kept in water systems and rooms hosting a range of other 
types of marine organisms, are not recommended. Additionally prey species should never be accommodated in the same tank as their predators.

In designing holding facilties for cephalopods and selecting the construction materials, it is recommended that guidelines developed for fish are followed (for review $\operatorname{see}^{79}$ ).

Materials used to build aquatic facilities should be non toxic. Any unavoidable use of material with the potential to be toxic should be reduced to the minimum, recorded and the information made available to staff, veterinarians and inspectors. In particular, materials that may release specific ions, chemicals or corrosion by-products from their surfaces should be avoided. The use of metals requires consideration of their interactions with seawater, and the potential effects of that interaction on the animals.

Special attention should also be given to the behavioural needs of the animals. For example, non-gregarious animals or animals that might show aggressive interactions (e.g. males during mating season) may require housing out of sight of others. Attention must also be paid to species-specific differences in terms of the level of disturbance that may be acceptable; for example, $O$. vulgaris appears to be quite resilient whereas cuttlefish or squid react more strongly to unfamiliar and sudden movements.

\subsection{Housing}

Cephalopods are strictly marine, and all require high-quality sea-water, but their varying habitats, social behaviour and especially nature and level of locomotion determine how they should be housed. Aquarium size and stocking density should be based on the physiological and behavioural needs of the individual species, and requirements for their health and welfare (see Appendix 2).

Section $3 \S 3 \mathrm{~b}$ of Annex III of the Directive indicates that all animals, including cephalopods, 'shall be provided with space of sufficient complexity to allow expression of a wide range of normal behaviour', including social behaviour, locomotion and feeding, and 'shall be given a degree of control and choice over their environment to reduce stress-induced behaviour'.

Stocking density will vary depending on the animals' natural history and behaviour, water flow, size, age and health. Water quality is critical (see section 4.1.2 above).

Most octopuses are solitary and should be kept in isolation. Nautilus are primarily solitary in the wild but may be housed together at low densities.

The social structures of many species, including the European cuttlefish ( $S$. officinalis) are not known, but in general social animals including many squid, are best kept in groups. However, social interactions should be monitored to check for adverse welfare effects; animals should be grouped according to age to avoid fighting and possible cannibalism, particularly in the breeding season or where there could be territorial antagonism. Such measures should not alter the overall welfare of the animal, and, in general, should be respectful of the behavioural needs of each individual species.

Depending on the species, individuals may require dens, shelters and other devices (mostly for bottomliving cephalopods).

Enriched environments must be provided, to allow the animals to express their normal behaviour (see further discussion of enrichment in section 5.11 below).

5.3.1 Tanks. Tank requirements and stocking density vary among cephalopod species and ages (see Appendix 2 for a summary of requirements). For example, for benthic species, like $O$. vulgaris and $S$. officinalis, the available bottom area is an important requirement, while for pelagic species this is represented by the volume of water; the depth of the tank should be considered for species with known diel (diurnal) vertical migration (e.g. Nautilus pompilius).

All cephalopods should be kept in opaque tanks of neutral colour. $O$. vulgaris and $S$. officinalis may also be kept exceptionally in transparent aquaria, as long as the floor of the tank is opaque (and/or covered by sand). Tanks can be of rectangular or of any other shape, but for decapod cephalopods they should have rounded corners to minimise potential injuries (see Appendix 2).

In general (and especially for Sepia and other decapods), sharp objects and rough surfaces that can cause skin damage must be avoided. Jetting can lead to collisions with the walls of the tank if animals are startled, or there is insufficient space for escape reactions.

Tanks can be made of PVC, fibreglass, glass or any non-toxic material capable of being adapted to achieve appropriate shapes and allowing a smooth internal surface, and which is easy to clean and sterilise or decontaminate as necessary. As potentially toxic materials might have been used during initial tank assembly (e.g. silicone-based adhesives and sealants), it is recommended to wash the tank thoroughly, leave it filled with water for at least 24 hours, and then rinse with seawater, before animals are introduced.

Tanks should be equipped with a covering (e.g. tank net or rigid transparent covering) that prevents animals escaping. Lids also serve as a barrier against the accidential introduction of any foreign objects, animals or chemicals. Tanks lids may be constructed of materials such as plexiglass or clear acrylic to allow visual inspection. The distance between the water surface and lid should be enough to minimise the risk of damage, for 
example, in the case of squid which are capable of leaving the water using their 'jet-propulsion'.

5.3.2 Tank labelling. Tanks must be carefully labelled, to identify and record the histories of individuals or groups of animals.

Labels should include detailed information for each individual, including origin, first dates in captivity and arrival in the laboratory, sex and morphometric measurements if possible (e.g initial body weight, dorsal mantle length), along with the number of animals in the tank (which may be an estimate depending on developmental stage).

For animals undergoing procedures, the label should identify: $i$. the procedures being performed (e.g. the label could refer to a detailed protocol filed for easy access by all relevant staff); $i$. the date when the procedures were started; iii. the person responsible for the animals (e.g. the Principal Investigator). Records of any adverse effects shown by the animals should be also be easily accessible 'tankside' and should be carefully maintained (see below for further discussion). Taken together, all these points should make it is easy for animal care staff and scientists to identify animals showing signs of welfare compromise, determine the likely cause of the adverse effects (e.g. whether procedures applied, such as recent anaesthesia) could explain the abnormalities, and take action to mitigate them.

\subsection{Cleaning of tanks}

Water quality should be monitored daily as a minimum (see also section 4). When water changes are necessary, the smallest possible amount should be removed.

Tanks should be free of organic waste (e.g. uneaten food or faeces), otherwise water quality, and thus animal health will be harmed.

Open systems: tanks should be regularly drained and cleaned to prevent fouling and reduced water exchange. There should be no risk of back-flushing, and consequent fouling of enclosure water. The sides and bottom of enclosures should be cleaned regularly to avoid the accumulation of detritus.

Closed systems: waste material should be removed as soon as possible after feeding. Total water replacement and whole tank cleaning should be avoided, as the biochemistry and flora that develop in a mature system are essential to well-being, as known in common practice for acquaria keeping. Depending on the size (i.e. number of tanks/system) care should be given to facilitate the most appropriate conditions at equilibrium. Where complete draining out of a system is required for decontamination reasons, the system must be allowed to remature after the addition of clean seawater, prior to adding animals.
When cleaning of tanks occupied by animals is necessary, the process should be designed to minimise disturbance and distress; in most cases animals will need to be removed from the tank during cleaning. Capture and transfer methods should conform to the principles outlined in these Guidelines, and the time spent in a holding tank should be minimised.

Disinfectants should be used with extreme caution and only in dry tanks, which are then rinsed with clean water. Detergents should be avoided and substitutes are preferred. ${ }^{120}$ Animals must not be exposed to any substance used for cleaning of tanks.

\subsection{Methods for individual identification and marking of cephalopods}

Depending on stocking density, it can be difficult to identify individual cephalopods. Marking or tagging, other than in species with external shells such as Nautilus, is difficult, owing to vulnerability to tissue damage. Individual cephalopods may have unique natural markings, and whenever possible these should be used for identification. ${ }^{121,122}$ Several marking methods have been successfully applied to different species of cephalopods (for examples see review in ${ }^{123}$ ). Methods used with success, but which require anaesthesia for their application - and hence scientific justification and approval from the National Competent Authority - have included implanted fluorescent elastomer tags in squid and octopus, ${ }^{124,125}$ subcutaneous dye injection into the arm of octopus, ${ }^{123,126}$ and external tagging of cuttlefish, octopus and other species. ${ }^{127-132}$

Careful consideration of harms, benefits and justification is therefore needed for invasive tagging, and development of minimally invasive individual marking methods for cephalopods is an important goal. ${ }^{31,133}$

\subsection{Food and feeding for adult cephalopods}

Most cephalopods are carnivorous and active predators, ${ }^{134-136}$ hunting their prey using a range of strategies (review in ${ }^{134}$ ). However, nautiloids are scavengers and some species of octopus will eat dead food items.

For many cephalopod species at different life-stages, live prey is the only known method of feeding. This prey may be fish or invertebrates, such as crustaceans, which need to be treated ethically and legally, ${ }^{137,138}$ and the feeding regime should suit the lifestyle, natural diet and developmental stage of the animals.

The duration of digestion (food intake to elimination) is $6-15$ hours in the common laboratory species of cephalopods and is slower at lower temperatures in a given species, ${ }^{139-141}$ so feeding frequency (and appetite) may alter with season (temperature) in open systems. 
Data on the richness of cephalopod diets in their natural habitats is limited, but known to include, amongst others, zooplankton, molluscs (including other cephalopods), polychaete worms, crustaceans, chaetognaths, sea urchins, fishes and jellyfish. ${ }^{26,142}$ An estimation of the relative breadth of diet has been attempted for some cephalopod species, including species most frequently used as laboratory animals, ${ }^{26}$ and shows that some species' 'natural' diets are restricted to certain prey items (i.e specialists), such as Spirula spir$u l a$, which feeds on detritus and zooplankton, ${ }^{143}$ whilst others are more opportunistic species (i.e. generalists) such as $S$. officinalis or $O$. vulgaris (for review see ${ }^{26}$ ). However, estimation of diet variety is substantially biased by research effort.

In laboratory conditions, animals usually adapt to prey on several different types of food. . $^{3,108}$

Nautilus requires food with a high level of calcium carbonate, such as shrimp with carapace, lobster moult shells or fish heads. Most cephalopods have a higher metabolic rate than fish, and their daily food intake which is rich in protein can be considerable: for example, $3-10 \%$ body weight per day. ${ }^{144}$ The feeding regime, palatability and method of food presentation should ensure that animals are adequately fed. Young and/or wild caught animals need particular attention. In some cases, enrichement with favoured foods and touching the animals' arms with food may trigger feeding.
Refusal to eat can be an early sign of illness (see also section 6.1.1).

Cuttlefish and squid are especially sensitive to inadequate nutrition; the most evident signs include: protruding eyes, poor body condition and floating (especially in juveniles). Consequently, in general over-feeding is preferred, as long as excess food is removed in an appropriate time-frame for the feeding habits of the species. ${ }^{145}$ However, ad libitum feeding of $S$. officinalis may cause buoyancy problems, so this is not advised (K. Perkins, unpublished data).

Artificial diets have been developed for cuttlefish and other species ${ }^{108}$ and are continuing to be explored in aquaculture research (Table 4; see also ${ }^{50}$ ). However, whilst an artificial diet may be ethically preferable and carries reduced risk of infection, studies to date indicate that growth and possibly welfare of the animals is reduced. ${ }^{146,147}$

The frequency of feeds is important and depends on the species and water temperature in the tank. The duration of digestion also depends on the species, and other factors including the animals' size, maturity and the type of food (for review see ${ }^{148}$ ). In O. vulgaris, gut transit times are quite rapid (about 12 hours at $18-19^{\circ} \mathrm{C}$ ) suggesting that crop capacity is not great and so daily feeding should be the norm. ${ }^{141,149,150}$ Daily feeding is also common practice for most coleoid cephalopods. However, other evidence from adult

Table 4. Use of alternatives and/or artificial/synthetic food to natural prey for rearing of some cephalopod species. The table is based on an overview of recent literature (most representative papers are included) mostly for aquaculture purposes (unless otherwise stated). For review see also Iglesias et al. ${ }^{50}$

\begin{tabular}{|c|c|c|c|}
\hline Species & Artificial food & Food item & References \\
\hline \multirow[t]{4}{*}{ Sepia officinalis } & Yes & $\begin{array}{l}\text { Pellet } \\
\text { Surimi }\end{array}$ & $508-510$ \\
\hline & No & Shrimps & e.g. ${ }^{511}$ \\
\hline & Yes & Lysine diet 4 & 151 \\
\hline & No & Natural frozen diet & 512 \\
\hline \multirow[t]{3}{*}{ Octopus maya } & Yes & $\mathrm{CPSP}^{\mathrm{a}}$ & 513 \\
\hline & Yes & Purina la $51 \%$ & 514 \\
\hline & Yes & Shrimp pellet + CPSP $^{a}$ & 515 \\
\hline \multirow[t]{4}{*}{ Octopus vulgaris } & Yes & $\begin{array}{l}\text { Moist pellets (fish and prawn mixed with alginate or } \\
\text { gelatin as binders) }\end{array}$ & $508,516-518$ \\
\hline & Yes & CPSP $P^{a, b}$ & 519 \\
\hline & Yes & $\begin{array}{l}\text { Diet S (50\% water, } 20 \% \text { gelatin, } 10 \% \text { egg yolk, } 5 \% \\
\text { S. aurita, } 15 \% \text { T. sagittatus) }\end{array}$ & 520 \\
\hline & No & Crustaceans; aquaculture by-products & $521-527$ \\
\hline
\end{tabular}

aCPSP: namely 'Concentrés de Protéines Solubles de Poisson' is a concentrated fish hydrolysate currently trademark of COPALIS (http:// www.copalis.fr/en/home/products-and-applications/animal-nutrition/aquaculture-breeding.html); see also Kristinsson and Rasco 528

${ }^{\mathrm{b}}$ This study is also aimed to the understanding of nutritional requirements of octopus 
cephalopods, particularly cuttlefish and octopus, suggests that they may not need to eat every day. ${ }^{146,147,151}$

\subsection{Food and feeding for larvae and hatchlings}

Evidence is provided that different dietary needs are required for cephalopod species during the early stages post-hatching. ${ }^{28,152,153}$ For example, hatchlings of $S$. officinalis often have a yolk sac which provides nutrition until they start feeding a few days later; in contrast, $O$. vulgaris paralarvae need to feed immediately in the water column. However, not all taxa of cephalopods have been successfully reared in laboratories, and so information on the dietary requirements of hatchlings is limited (for review see ${ }^{50}$ ). Evidence available for some species suggests that embryonic development often requires trace nutrients that are present in natural seawater, ${ }^{154,155}$ in which case development might be hindered in closed artificial systems. It has also been suggested that 'dissolved gases and nutrients may also contribute to metabolic and nutritional requirements via absorption through the epidermis'. ${ }^{144,156}$ In addition, a close relationship between the fatty acid profile of the dietary components and of the individual at early stages after hatching has been reported. ${ }^{157}$ This emphasises the importance of improving understanding the nutritional needs of juveniles, especially if artificial diet is being considered for rearing purposes.

\subsection{Handling and moving cephalopods in the laboratory}

Handling procedures should be carried out only by competent, trained personnel using techniques that minimise the potential for injury and reduce stress to the animals (see also section 10). It is recommended that laboratory coats and gloves should not be of white/pale colours, as the handler can be mistaken for a 'predator'.

The skin of cephalopods acts as an organ ${ }^{158}$ and is very delicate and so every effort should be made to minimise handling and removal of animals from the water. It is especially beneficial to standardise handling procedures, as anecdotal evidence indicates that cephalopods can habituate to handling.

It is preferable to move the animals in water using containers where they can be gently restricted before moving from one tank to another or any other location. Training animals to enter a container, possibly using small rewards, may reduce stress and habituate them to the transfer.

Cuttlefish and squid should be immersed at all times and a dark net should only be used to coax the animal into a container.
Nautilus is particularly sensitive to air, and repeated air exposure is anedoctally reported to have negative effects on the health of the animals (R. Smallowitz, unpublished data).

Octopuses can be moved using nets (suggested dark nylon 2-mm mesh) with a long sleeve to reduce the risk of escape; exposure to air should be minimised. A container method has been developed for O. maya ${ }^{159}$ and, although not currently in use, represents a useful approach indicative of methods that should be developed for animal transfer within a facility.

Nets and containers should be clean, disinfected and rinsed before use. Agitation during moving should be minimised, as all cephalopods have a sensitive statocyst system. ${ }^{160}$

Handling and other human interactions should be monitored and recorded, as the frequency and nature of the interactions can influence behavioural performance of individual animals.

\subsection{Environmental enrichment}

Environmental enrichment should not compromise the need for adequate levels of hygiene and the ability to observe the animals' health (section 6 below) without causing too much disruption. The impact on health and welfare of environmental enrichment should be evaluated objectively, ${ }^{161-163}$ particularly to avoid the application of 'environmental changes' which may be detrimental to the animal well-being, and to ensure health or water quality are not compromised.

Section $3 \$ 3 b$ of Annex III of the Directive states that 'Establishments shall have appropriate enrichment techniques in place, to extend the range of activities available to the animals and increase their coping activities including physical exercise, foraging, manipulative and cognitive activities, as appropriate to the species. Environmental enrichment in animal enclosures shall be adapted to the species and individual needs of the animals concerned.' The same section also states that 'the enrichment strategies in establishments shall be regularly reviewed and updated'.

These provisions require on-going consideration of the effects of laboratory housing on animal welfare and efforts to enhance well-being wherever possible. Exemptions from these, and other, requirements outlined in Annex III have to be approved by the National Competent Authority, and must be for scientific, animal welfare or animal health reasons.

Environmental enrichment aims to enhance the wellbeing of animals in captive conditions, by identifying and providing stimuli that enable animals to express as wide a range of their normal behaviours as possible. ${ }^{164,165}$ Enrichment is proven to be effective for 
many species, including fishes, ${ }^{166-168}$ cephalopods and other invertebrates. ${ }^{138,169-175}$

Enrichment may be accomplished through changes in the tank environment, for example, by varying factors, such as the shape of the tank, flow of water, variety of live prey items (if these are essential), conspecifics and environmental complexity; and also by providing opportunities for animals to engage in specific activities and exercise some choice.

Enrichment strategies should be tailored to the needs of the particular species concerned. For example, openwater species may require large but less complex environments. Social animals should be housed in groups. Benthic cephalopods are better kept in complex environments with suitable substrates (sand, gravel or pebbles) and dens.

Nautiluses should have access to vertical space for movements and attachment at a variety of levels, thus meeting their natural habit of daily vertical migrations. ${ }^{176,177}$ However, not too many vertical attachments should be added, as nautiluses naturally swim up and down whilst circling around the perimeter of tanks and require space to do so. Adding texture (artificial coral reef) to at least one wall of the tank may make it more attractive to the animal (and may promote egg laying; G. Barord, pers. comm.).

In octopuses, interaction with objects is a common form of enrichment and is recommended; providing a den as refuge is not considered to be enrichment, as it is a basic requirement for octopuses, and for all benthic species that use refuges in the wild. Artificial shelters can take the form of many different objects (e.g. bricks, ceramic pots, plastic jars), but dark and opaque dens are preferred over clear ones.

Suggestions for the type of objects (artificial and/or natural) to be added in tanks as enrichment for most common cephalopod species are provided by Grimpe. ${ }^{56}$ Recent systematic studies are missing and data available are mostly anedoctical.

Caution should be taken to avoid objects added to holding tanks that could harm or limit full expression of the behavioural repertoire of the animal. Mirrored surfaces should be avoided, since may create agonistic reactions expressed by some individuals towards the 'ghost' reflected image (G. Fiorito, pers. comm.). Accounts of tank design for coastal and reef squid species provide also information on environmental enrichment for these animals. ${ }^{76,178}$

\section{Assessment of health and welfare}

Annex III, Article 3.1 of the Directive requires that establishments have a strategy to ensure that the state of health of the animals safeguards animal welfare and meets scientific requirements. This should include regular health monitoring and plans for dealing with health breakdowns (see section 7). The starting point for fulfilling these requirements is objective monitoring and recording of the health and welfare of the animals and recognition of the factors likely to cause deviations from optimal status.

The primary factors that could cause a decline in health and welfare and which require monitoring are effects of:

- environmental and housing conditions (including: capture, transport, handling, stocking density, tank design); nutrition; variations in: water temperature, oxygen levels, $\mathrm{pH}$, salinity and water contaminants (sections 4 and 5);

- infectious disease (section 7); and

- experimental (regulated) procedures (section 8).

Irrespective of the cause, objective criteria for assessment of the overall health and welfare status of animals are required to:

(i) ensure that animals arriving in the laboratory are healthy;

(ii) ensure that housing and care are adequate for the maintenance of good health and welfare;

(iii) assess the impact of experimental procedures (section 7) in terms of severity and identification of pre-established humane end-points;

(iv) identify and implement measures to rectify health and welfare problems and enhance the well-being of animals, and refine procedures so that they cause less harm to the animals; and

(v) monitor the efficacy of any therapeutic interventions.

As for other animals, the key parameters used to assess the health and welfare of cephalopods are behaviour and appearance, supplemented in some cases by measurement of a number of physiological 'biomarkers'.

Animals should be inspected immediately on arrival in the laboratory and at least daily thereafter; a consistent method should be used for recording observations, evaluation and actions to be taken modified as required for each species. An example of the types of observation that could be made is shown in Table 5 and provides a starting point for the development of species specific observation sheets. Oestmann et al. ${ }^{145}$ recommend that general behaviour, indicative of well-being, in cephalopods is assessed 2-4 times per day; and G. Fiorito (pers. comm.) recommends at least twice a day.

For animals that are being used in an experimental (regulated) procedure (section 8), the observation 
듕 $\stackrel{0}{ \pm} \stackrel{0}{ \pm}$

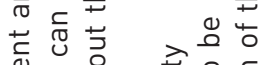

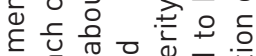

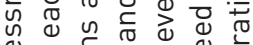

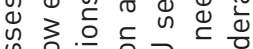

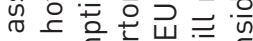

त्रे है

중 든

$\subseteq 0$ 응응

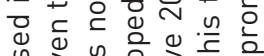

光긍

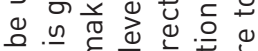

응

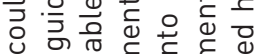

$+\pi+v_{0}$

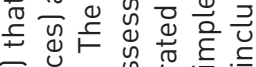

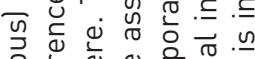

은

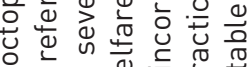

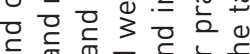

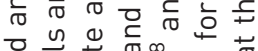

윽

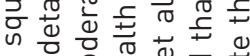

जٓ

包比

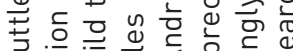

己

근

일

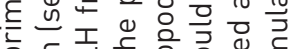

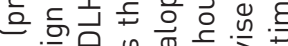

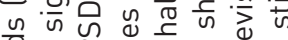

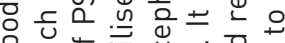

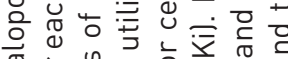

ฮั

눙

u之ं

$\subseteq$ 든

ब

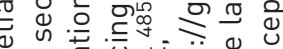

$30 \frac{\pi}{\pi}$

으웝

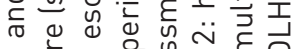

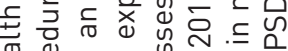

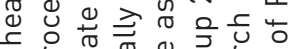

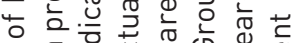

ज

잉

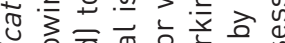

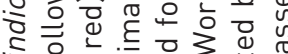

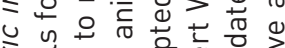

凶

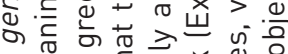

을

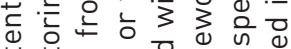

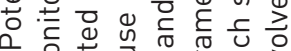

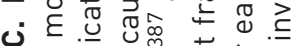

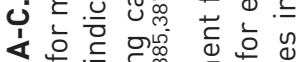

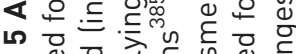

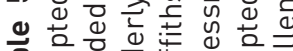

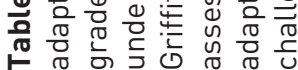

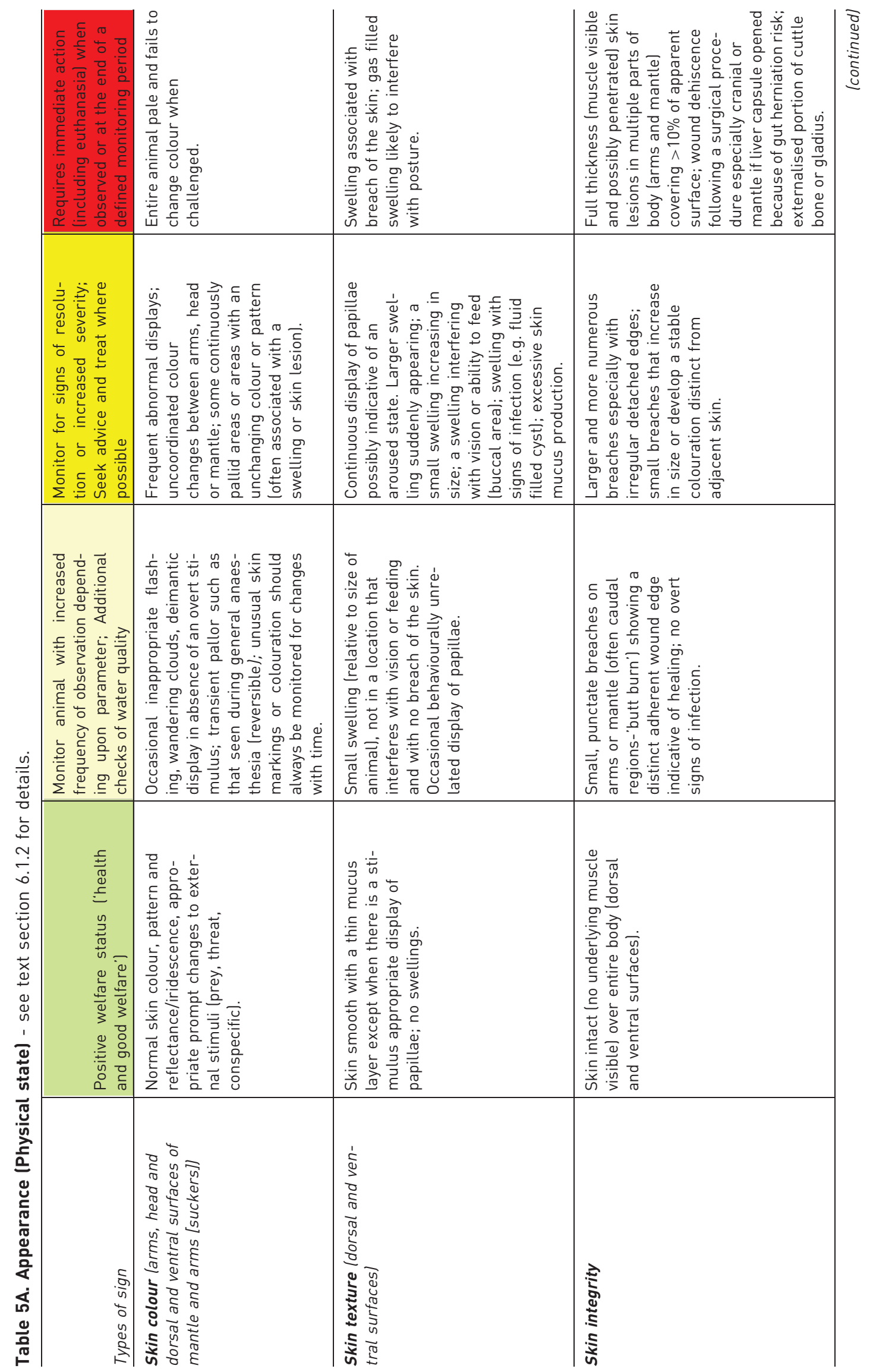




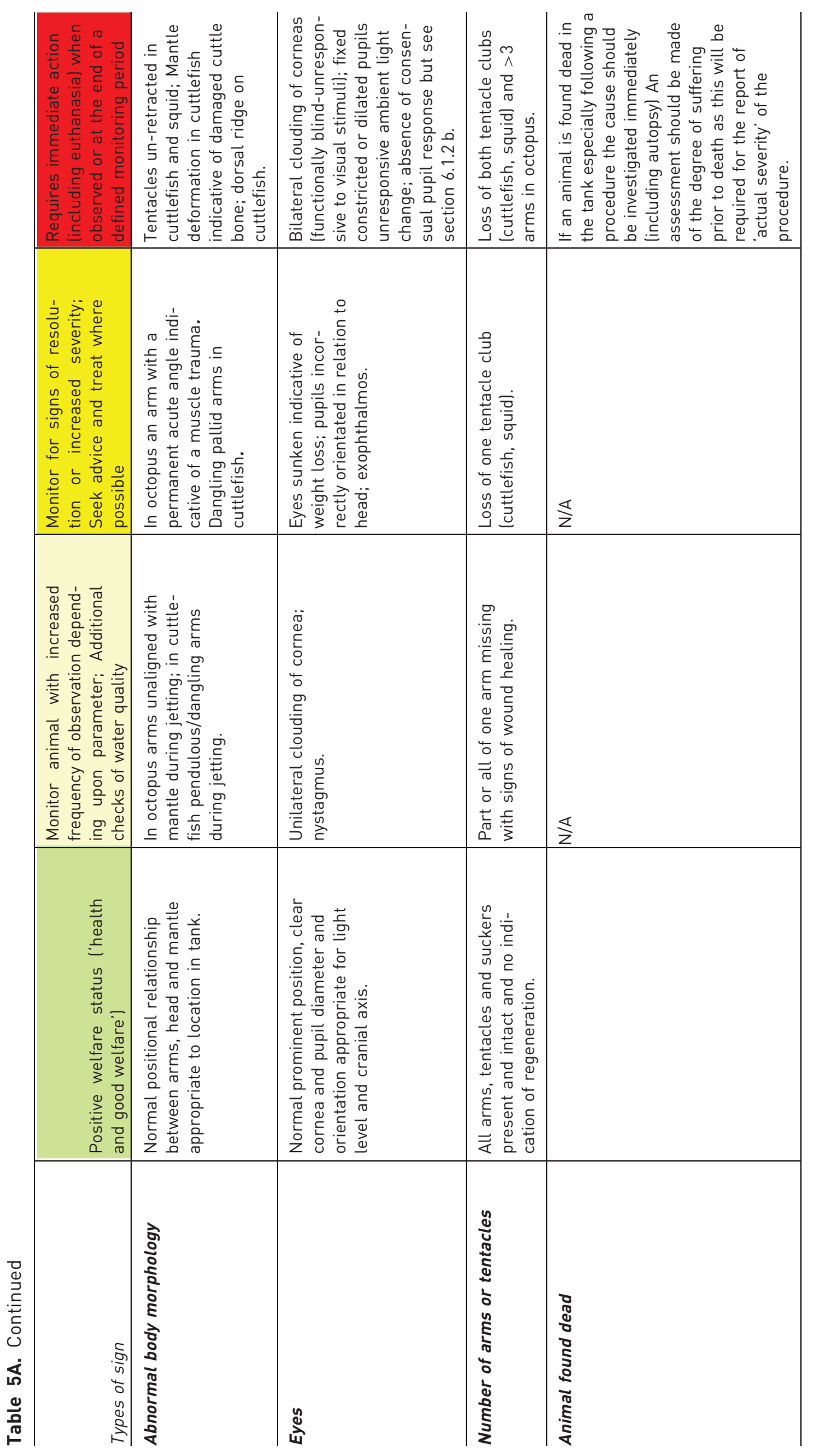




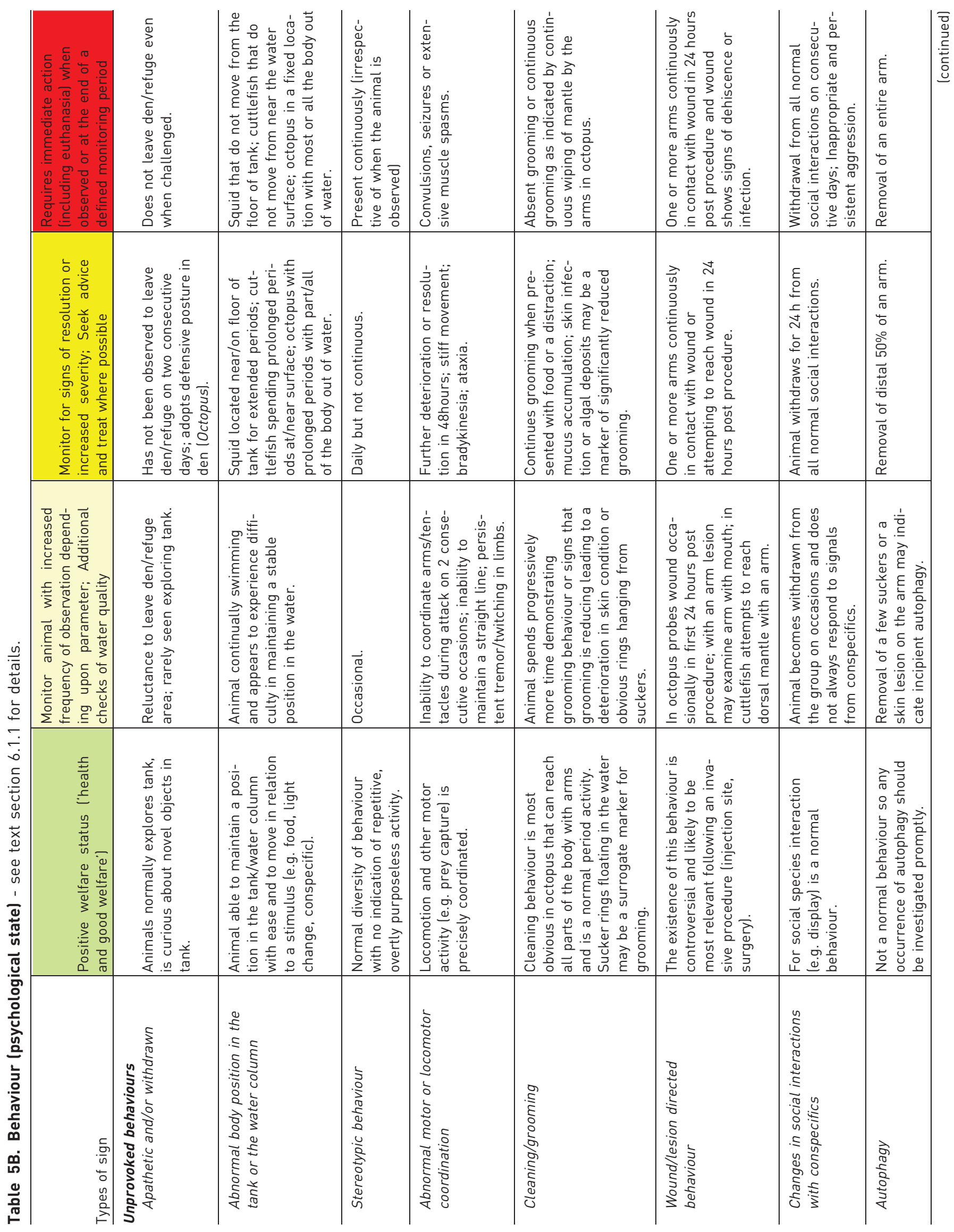




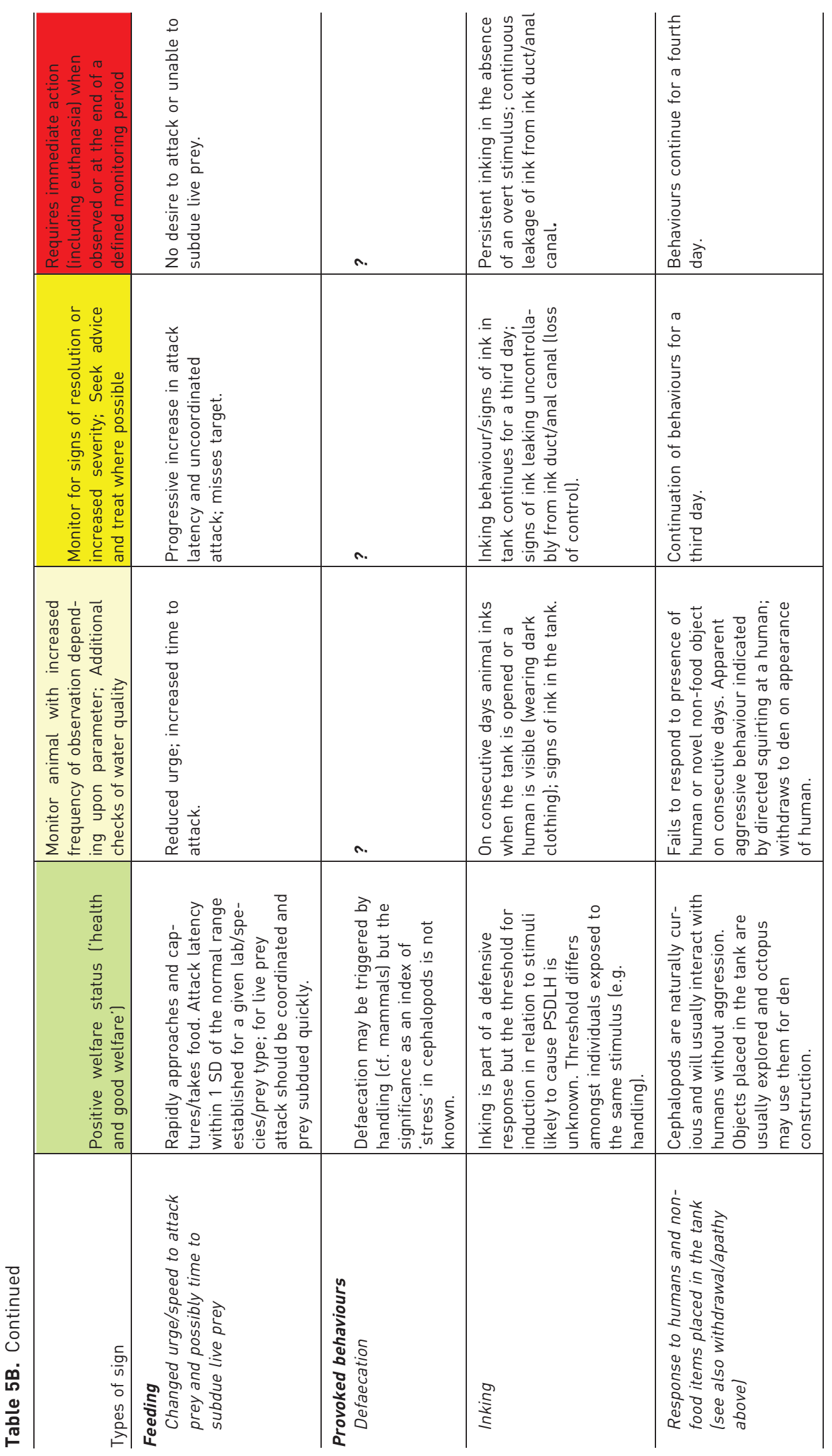




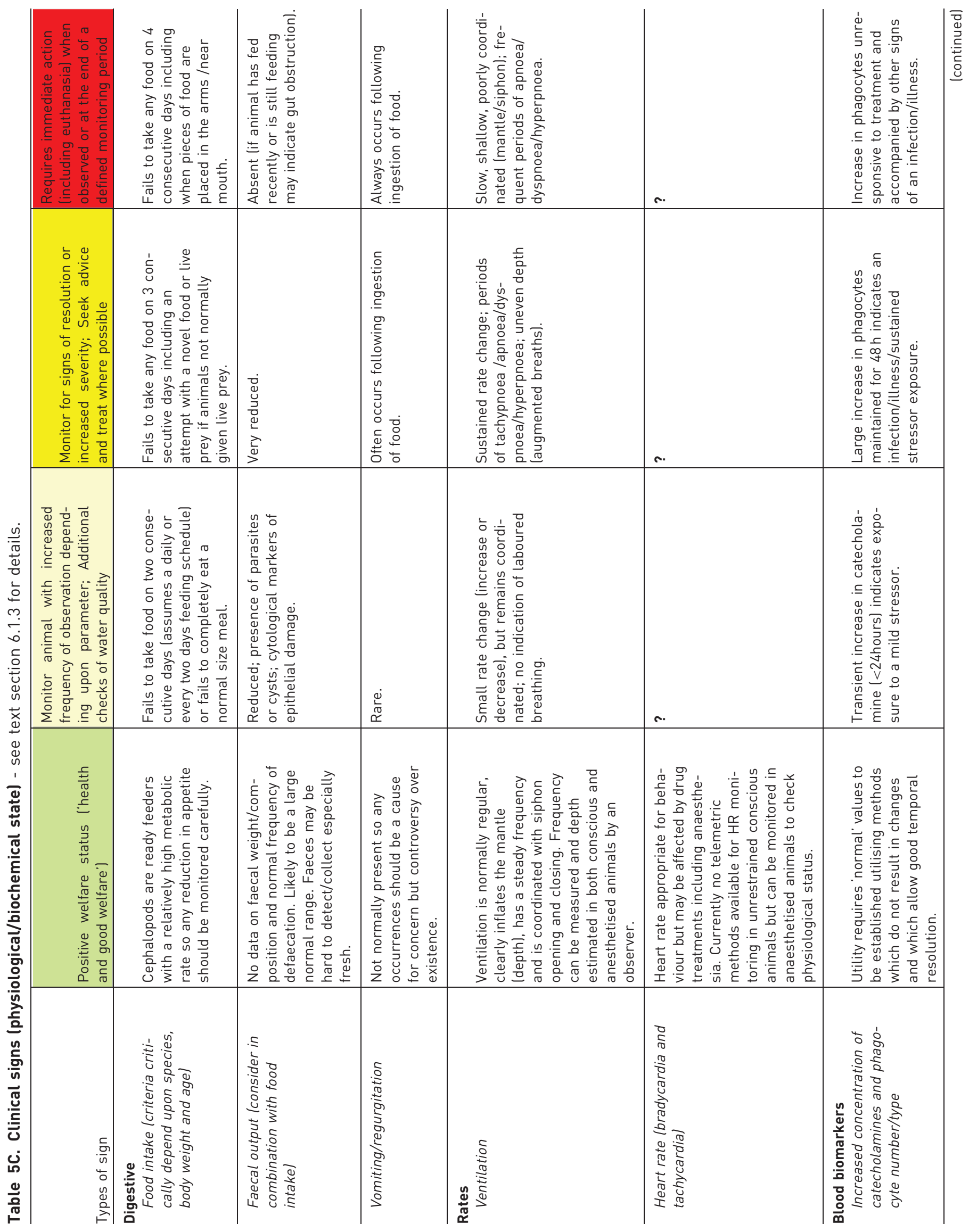




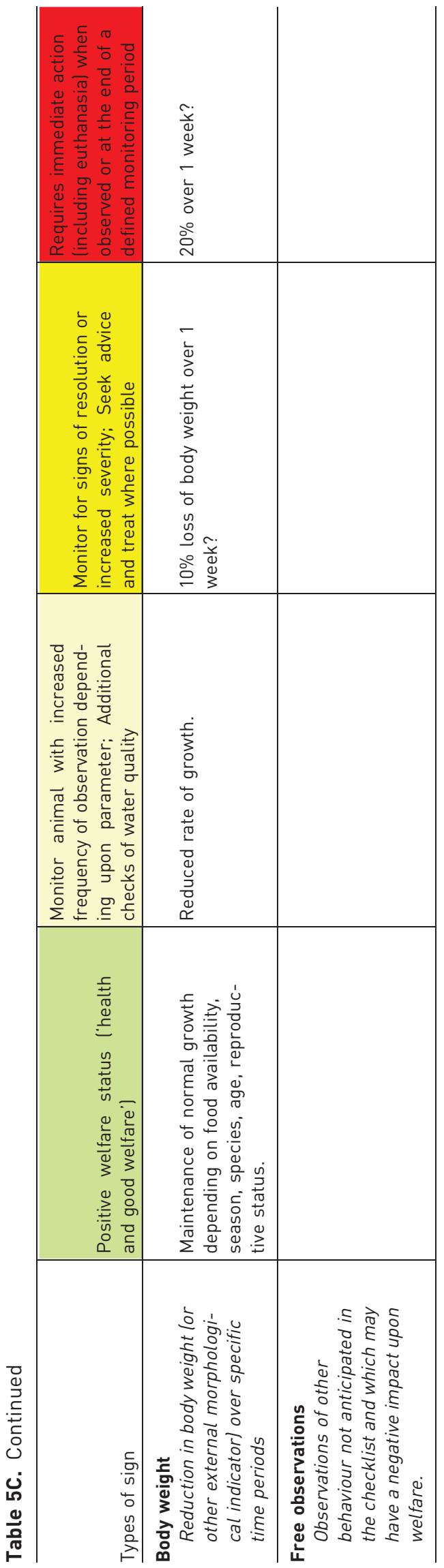

frequency may be increased depending upon the nature of the procedure and its anticipated impacts on animal welfare. Factors to be considered are described in detail below, but most require further validatation, including consideration of their severity (see section 2.2.3 and 8.2).

It should be noted that prompt identification of problems is essential so that $i$. action can be taken to reduce and preferably eliminate any suffering and $i i$. in the case of procedures, to ensure that humane end-points are promptly implemented where appropriate and that severity limits are not exceeded (sections 2.2.3 and 8).

\subsection{Objective assessment of health and welfare}

Proposed parameters are described in detail below and summarised in Table 5. Welfare assessments should be performed in the animals' home tank and without removal of the animal from the water wherever possible. Each element of the assessment will ideally require some form of quantification to enable recognition of points at which particular parameters reach a pre-set humane end-point and to enable actual severity of a procedure to be reported.

Observation and evaluation of the following criteria can help to determine whether 'something is wrong' with the animals, and, considering the overall pattern of observations, can help decide strategies for rectifying any health and welfare problems.

\subsubsection{Observation of spontaneous and provoked animal behaviour}

a) Feeding. The common laboratory species of cephalopod have voracious appetites and eat relatively large amounts of food in relation to body weight, reflecting a relatively high metabolic rate.

Therefore, altered feeding behaviour manifests as a reluctance to take food or an increase in the time to attack or subdue live prey, and is usually the first and most apparent behavioural indicator that there could be a health or welfare problem. For example, in octopuses fed on crabs or mussels, the tank should be checked for empty carapaces and shells to ensure the animals are ingesting prey and not just attacking them. In $O$. vulgaris the willingness to attack can be tested equally well with an artificial crab as with a live crab. ${ }^{179}$

b) Location in the tank and water column. Each species normally locates in a characteristic place in the tank and water column.

Nautiluses spend much of their time attached to the sides of tanks, and undergo daily vertical migrations 
(see above), so animals spending large amounts of time at the bottom of the tank other than when engaged in feeding are exhibiting abnormal behaviour.

Cuttlefish alternate between hovering/swimming in the water column and resting on the bottom, partially covered by the substrate, and an animal spending a considerable period of time at or near the surface of the tank should be inspected closely for signs of physical damage to the mantle. Such changes have been observed following a pharmacological treatment, as described by Agin et al., ${ }^{180}$ as a consequence of cycloheximide injections.

Squid rarely rest on the bottom of the tank, so their presence there for an extended period should be regarded as abnormal, as should extended periods spent at the surface.

For octopuses, a 'problem' in the tank may be indicated if the animals spend excessive time clinging to the lid of the tank with most of the body out of the water.

c) Swimming and locomotor activity and coordination. Each species has a characteristic method of moving in the tank: by walking, swimming or a combination of both. ${ }^{31}$ Repetitive locomotion in cephalopods, such as jetting backwards continuously, or in the case of octopuses performing swimming motions while attached to a tank wall, can be a sign of stress. Any abnormalities of coordination should be noted. For example, a defect in the statocyst leads to an inability to control orientation during swimming. This 'spinner' behaviour has been reported in species of cuttlefish, squid and octopus. ${ }^{181}$ Although there are likely to be minute-tominute changes in the level of locomotor activity (see e.g. Figure 1 in Boyle ${ }^{182}$ ), each species has its own overall daily pattern of activity cued by the photoperiod. ${ }^{98,100,130,183-186}$ Rest/sleep-like-activity cycles are documented in S. officinalis, ${ }^{102} \mathrm{O}$. vulgaris $^{101}$ and Octopus macropus $;{ }^{103}$ nocturnal vertical migration is known to occur in $N$. pompilius. ${ }^{29,177}$ Changes could be an indication that 'something is wrong'.

d) Use of arms and tentacles. The behavioural repertoire of arm movements in cephalopod species is reviewed and described in Borrelli et al. ${ }^{31}$ A taxonomy of arm movements for octopuses is provided by Mather. ${ }^{187}$ An animal spending an extended amount of time with the arms curled over the body (a defensive posture), either in the den or the corner of the tank, should be monitored for other indications of distress. In Nautilus withdrawal of all tentacles into the shell with the opening obstructed by the hood is a defensive behaviour. ${ }^{188}$ There are scattered reports of octopus using an arm to 'guard' an injured part of the body (I. Gleadall personal observation cited in; ${ }^{8}$ reports of animals 'guarding' the mantle or cranium post-surgery by G. Fiorito, unpublished data, and also ${ }^{189}$ ), but this behaviour has not been systematically investigated and could also be linked to facilitation of healing by secreted antimicrobial peptides. Wound-directed behaviour was not observed in a study of tentacle amputation in the squid Loligo pealeii, ${ }^{9}$ although in two species of cuttlefish (S. officinalis and S. pharaonis), the use of a partially (80-90\%) amputated arm for prey manipulation and body posturing was avoided for up to 3 days post lesion. ${ }^{190}$

In cuttlefish, reaching over the dorsal mantle ('scratching-like' behaviour) has been observed a few days after a transient rise in ammonia concentration leading to skin damage. ${ }^{145}$ Tentacles in cuttlefish and squid should be retracted except when the animal is engaged in an attack.

The arms are used for skin cleaning in octopus; excessive cleaning activity and/or frequent presence of sucker-rings in the tank could be indicative of abnormality. In all cephalopods, a loss of adhesion in the suckers should be a cause for concern.

e) Interactions with humans and conspecifics. While cephalopods, in general, are very responsive to any novel features introduced in their tank, octopuses are especially curious about their environment. Healthy octopuses acclimatised to laboratory housing will often leave their den when the tank is inspected and will interact with a hand placed below the surface of the water (for an historical account see $\left.{ }^{191}\right) .^{36,192}$ Reluctance to interact with humans should be a cause for concern. It should also be noted that there is some evidence that at least one species of octopus (E. dofleini) may recognise individual humans; ${ }^{193}$ therefore, care should be taken to ensure that staff who are involved in any procedure likely to be aversive should not be involved in routine feeding or inspection as there is a possibility of inducing a conditioned aversion/ avoidance.

If visually exposed to conspecifics, octopuses may alter their predatory response due to agonistic interactions, but habituation resulting in a resumption of normal behaviour has been observed under controlled laboratory conditions. ${ }^{194,195}$ Squid and cuttlefish are social species and changes in social interactions with conspecifics again may indicate a welfare/health problem.

f) Squirting, inking, defaecation and regurgitation.

Squirting: all cephalopods use expulsion of water from the mantle via the siphon in breathing and locomotion; and this is particularly noticeable in the jetting escape reaction. Squid and cuttlefish 
may direct jets of water at a person attempting to capture them, and this behaviour is particularly notable in $O$. vulgaris where jetting is also a component of the deimatic display. ${ }^{31,134}$ Water jets directed at an observer is indicative of a moderate aversive reaction; in some cases this can also be a sign of 'recognition'. ${ }^{195}$

Inking: is a defensive response in cephalopods (apart from nautiloids which do not have an ink sac), so inking should always be taken as an indication that the animal perceives a threat or is stressed. However, there is individual variability in the threshold for induction of inking as some $O$. vulgaris will ink profusely in response to handling (M.G. Valentino and P. Andrews, unpublished data) that does not evoke the same response in other individuals (G. Fiorito, pers. comm.). In addition, inking does not necessarily result from the animal receiving a presumed noxious stimulus, such as an electric shock (I. Gleadall personal observation cited in $;^{8}$ G. Fiorito, pers. comm.). Therefore, absence of inking should not be interpreted as an absence of anxiety or distress. A continuous trickle of ink from the animal should be investigated as it may indicate a problem with the neural control mechanism or with ink duct sphincter competence. Animals should not be allowed to remain in a closed tank in which inking has occurred, and care should be taken to ensure that ink does not enter other tanks as it is an alarm signal. ${ }^{196}$ Intramantle inking has been reported as a post-transport stress behaviour in Octopus bimaculoides. ${ }^{197}$

Defaecation: there is insufficient knowledge of defaecation patterns and their control in cephalopods to determing whether any changes may be linked to pain, anxiety or stress, as is the case in many vertebrates. Although faecal ropes may emerge in octopus exposed to general anaesthetics, this could be due to loss of anal sphincter control. The production of faecal ropes in octopus is a useful indicator of normal digestive tract functioning in a feeding animal, but it is not known whether disease can alter the faecal fluid content or defaecation frequency (constipation/diarrhoea). However, chemical and cytological examination of fresh faecal samples can provide important insights into the health of the animal and as collecting faecal samples is non-invasive, its utility in cephalopod health monitoring should be explored.

Regurgitation: there are two isolated observational reports of regurgitation/vomiting of upper digestive tract contents one in E. dofleini (I. Gleadall personal observation cited $\mathrm{in}^{8}$ ) and the other in Sepioteuthis sepioidea. ${ }^{198}$ The location of the beak within the crown of arms would make this behaviour very difficult to detect. However, if the ability to regurgitate/vomit upper digestive tract contents is confirmed then it should be added to the list of possible indicators of illness, as is the case in vertebrates. ${ }^{199}$

\subsubsection{Appearance}

a) Skin colour, pattern and texture. Skin colour and pattern are primarily regulated by motorneurones from the suboesophageal chromatophore lobes of the brain, with contributions from reflecting cells, depending upon the location on the body and the species. ${ }^{134,200-202}$

At the time of writing, there is no evidence to show that changes in the colour or pattern of the skin in any cephalopod species are specifically associated with changes in health or welfare of the animals. Oestmann and coworkers ${ }^{145}$ caution that normally functioning chromatophores and iridiocytes may mask underlying skin defects. In Nautilus discoloration of the mantle (with loss of buoyancy) is a sign of poor health. ${ }^{203}$ However, loss of ability to match substrate or background (see e.g. ${ }^{204-207}$ ) or sustained pallor with loss of normal patterning should be taken as an indication of a problem, as should excessive or inappropriate flashing in squid $^{208}$ and wandering clouds in coleoid species. ${ }^{209}$ Note should also be taken of colour changes in response to a provocative stimulus, such as the deimantic display often observed in response to a perceived threat. Skin texture in octopuses and cuttlefish can be changed by the formation of papillae, particularly prominent above the eyes and on the mantle and is indicative of an aroused or vigilant animal. ${ }^{31}$

b) Skin and external shell integrity. Any breach to the skin of a cephalopod is potentially problematic because of the possibility of bacterial infection (section 7.2.2) causing systemic sepsis, preventing healing, and local inflammation causing hyperalgesia. Bleeding from wounds may not be readily apparent as, although oxygenated haemolymph is pale blue (extracellular haemocyanin), it will be rapidly diluted in the tank and deoxygenated haemolymph is colourless.

Healing of small wounds in octopuses, such as transection of the distal $10 \%$ of the length of the arm, appears to be rapid, with the exposed area in some animals being almost completely covered by skin in about 24 hours (T. Shaw and P. Andrews, unpublished data), but larger wounds and particularly those to the mantle appear to take longer to heal even without infection. ${ }^{210}$ Damage to the skin most frequently occurs at the distal part of the mantle in 
animals (particularly squid and cuttlefish) that frequently impact the wall of the tank ('butt-burn': J. Rundle, pers. comm.), with four impacts per hour being recorded in a study investigating the longterm health of cultivated cuttlefish in soft-sided tanks. ${ }^{211}$ In cuttlefish and octopus, the ventral surface of the mantle contacting the substrate should also be examined, and anecdotal evidence that ill or senescent octopuses avoid rough substrates may indicate that the skin in this region is particularly sensitive to damage. Animals showing signs of healed skin damage should be inspected closely to ensure that it is healing, as death may ensue rapidly if the lesion increases in size and penetrates the underlying muscle. ${ }^{212}$

Breaks in the shell of nautiloids may compromise their buoyancy mechanism and so require treatment.

The cause of any breach in skin or shell occurring after an animal's arrival in the laboratory should be identified and action taken to prevent recurrence (e.g. carefully examine the tank and items in it for sharp edges, deep clean and disinfect the tanks, see section 5.6).

c) Eyes. The eyes should be inspected to ensure that the cornea and lens are transparent, as opacity is one of the signs of natural senescence (see below). Pupil diameter should decrease over a few seconds in response to a sudden increase in illumination (for Nautilus see; ${ }^{213}$ for cuttlefish and octopus; ${ }^{214}$ for squid $^{215}$ ), although there is some evidence ( $S$. officinalis and E. cirrhosa) that the response is not consensual. ${ }^{214}$ The classic contributions of Beer $^{216}$ and Magnus ${ }^{217}$ should be also considered in this framework. The slit-like pupil remains close to horizontal irrespective of the position of the body, and this is particularly noticeable in octopus. ${ }^{150}$ Both the pupil diameter and statocyst-ocular responses (nystagmus) are mediated by the brain and hence give an insight into central nervous system functionality. ${ }^{181,218-220}$

d) Body posture. Two aspects need to be considered: $i$. the relationship between the mantle, head and arms/ tentacles (i.e. the overall appearance of the animal), and $i$. the orientation of the animal in relation to the floor and sides of the tank.

In Nautilus the shell should be vertical; however, air bubbles can become trapped in the eyes and under the hood, leading to adverse health effects. ${ }^{221,222}$ Information on treatments related to poor health conditions is available in Barord et al. ${ }^{223}$ Trapped air can be released by slowly turning the animal, laterally from side to side.

All cephalopods have well-developed statocyst systems to maintain body posture and coordinate body position with eye orientation ${ }^{224}$ so an abnormal body posture may indicate a nervous system problem or a physical defect that the animal cannot compensate for (e.g. a broken cuttlebone, a fluid-filled chamber in Nautilus, gas trapped in the distal mantle of octopus). Damage to arms can also affect posture as Tressler et al. ${ }^{190}$ reported unbalanced swimming (body axis tilted to the lesioned side) lasting up to 3 days in cuttlefish in which 80 $90 \%$ of the length of third right arm was removed. The head in octopuses is particularly mobile and a raised head, particularly if moving from side to side or bobbing has been regarded as sign of 'agitation' in O. vulgaris by Boyle. ${ }^{182}$ However, head bobbing and similar behavioural patterns are indicative of increased arousal, as reviewed by Borrelli and coworkers. $^{31}$

\subsubsection{Biomarkers}

a) Body weight. The optimum frequency with which animals can be handled for routine weighing, taking into account that anaesthesia may be necessary, is not known. It has been suggested ${ }^{225}$ that frequent handling may impede growth, but this requires systematic investigation. Cephalopods, particularly when young, increase body weight daily (assuming sufficient food) so failure to increase weight or a loss of weight following an experimental procedure may be the earliest objective measurement of declining health or welfare, but the potential additional harms of frequent weighing will need to be assessed.

Dorsal mantle length (DML) is also frequently used as an index of body size in cephalopods although the relationship to body weight (TBW) is not linear. The $K$-Fulton condition index, which combines length and body weight measurement and is used in fish, has been adapted for cephalopods $\left(K=\left(\mathrm{TBW} / \mathrm{DML}^{3}\right) \times 100\right) .{ }^{226}$ In $O$. vulgaris infected with Aggregata octopiana, the $K$-Fulton condition index decreased as the sporocyst counts in the caecum increased. ${ }^{226}$ Consideration should be given to using this index as part of routine growth and health and welfare monitoring in cephalopods, as applied to many species of fish, ${ }^{227-233}$ and in other circumstances to other vertebrates (see, e.g. ${ }^{234,235}$ ).

In stock animals, weekly measurement of body weight may also be a useful index of health and welfare status, providing this can be done with minimal distress to the animal (e.g. in seawater), but the percentage loss of weight over time that is indicative of illness is not known. The digestive gland has a 
lipid reserve, ${ }^{236}$ Mangold and Bidder estimated as $9-13 \%$ of digestive gland weight in $S$. officinalis. ${ }^{148}$ However, the impact of food deprivation on this is not known.

Growth data based upon body weight are species and laboratory specific with the latter depending upon food type and feeding frequency, water temperature, stocking density, animal age, activity level (influenced by tank size and photoperiod) and parasite load. There are limited growth curve data for representative cephalopod species; for example for: Nautilus, ${ }^{237}$ cuttlefish and squid, ${ }^{238}$ and octopus. 225,239

b) Ventilation (breathing) frequency. Ventilation frequency can be monitored by an observer provided that the animal is not disturbed, but a video system may provide an alternative in the absence of other non-invasive methodology, as standardised by Borrelli. ${ }^{26}$ Although an increase in ventilation frequency may be an indication of physiological stress (e.g. particularly a fall in inspired $\mathrm{pO}_{2}{ }^{84,240,241}$ ), it is also indicative of arousal to innocuous stimuli and ventilation frequency also correlates positively with activity levels, as for $S$. officinalis and $O$. vulgaris. ${ }^{182,242}$ Observation of breathing should also note whether the pattern is even or is interspersed with periods of apnoea/tachypnoea.

Although frequency is relatively easy to monitor, ${ }^{26}$ some assessment should also be made of depth (mantle stroke volume) as again excessively deep, shallow or laboured breathing may also indicate a problem and Smith et al. comment that maintenance of oxygen uptake (in $O$. vulgaris) relies more on stroke volume than increasing ventilation frequency. ${ }^{240}$

Deep/forceful breathing may manifest as currents in the water or ripples in the surface if the animal is close. In the coleoids, the way in which the mantle distends during inspiration should be noted to ensure it is bilaterally symmetrical, that the entire mantle is involved, and inspiration and expiration are coordinated with the closing and opening of the siphon respectively. During general anaesthesia, ventilation frequency, depth and coordination all become suppressed. ${ }^{243}$ Therefore, similar changes in a non-anaesthetised animal are likely to reflect depression of brain drive and should be investigated immediately.

Stress state in Nautilus is usually expressed as a 'rocking behaviour' (which reflects hyperventilation), with the animal clearly rocking from front to back.

c) Cardiovascular parameters. Currently there is no established non-invasive method for routine measurement of heart rate or blood pressure in an unanaesthetised cephalopod (but $\mathrm{see}^{244,245}$ ). In addition, studies of animals with indwelling catheters show that both parameters are very labile ${ }^{150}$ suggesting that, even with appropriate methodology, they may not be helpful as indices of health or welfare. In particularly compliant individual $O$. vulgaris, it is possible to observe the beating of the systemic and branchial hearts in the mantle without anaesthesia, and to use Doppler ultrasound to investigate cardiac function and image the viscera (G. Ponte, pers. comm.). The resolution achieved is much improved from previous attempts with cephalopods. ${ }^{244,246-248}$ This potentially represents a revolution for future physiological studies with these animals (D. Fuchs and G. Ponte, unpublished data; Vevo 2100 Visualsonics, The Netherlands). Such methodology may be suitable for detailed investigation of animals showing signs of illness and could be useful for monitoring some physiological functions during general anaesthesia.

d) Other biomarkers: analysis of blood. There are no validated blood biomarkers indicative of the health or welfare status of a cephalopod, and the development of such markers is hampered by difficulty in obtaining blood samples using minimally invasive techniques comparable to those available for vertebrates (but see also Table 8). Descriptions of blood sampling in the literature employ some form of general anaesthesia as, for example, done in the bobtail squid E. scolopes. ${ }^{249}$ In animals sedated for investigation using a low concentration of a general anaesthetic (see section 8.5.5), blood sampling and analysis may be a helpful aid to diagnosis and treatment but the relative harms and benefits of undertaking this procedure solely for welfare assessment need to be considered.

The following parameters should be considered.

Haemocytes. Blood samples enable culture for bacteria and examination of smears by electron microscopy for viruses. The utility of haemocyte counts and morphology in general and phagocytes specifically as indicators of infection or stress is limited because sampling methods (especially repeated sampling involving anaesthesia) themselves seem to increase haemocyte concentration, ${ }^{250,251}$ although the concentration is increased further by bacterial infection the effect is transient (i.e. present at 4 but not 24 hours in E. cirrhosa). ${ }^{126}$ The increase in haemocyte counts in response to intramuscular injection of Escherichia coli lipopolysaccharides in $O$. vulgaris begins within 4 hours of injection and is returning to control levels by 24 hours. ${ }^{251}$ Vehicle injection (phosphate buffered saline) produced a smaller increase in 
haemocytes indicating that a rise in haemocytes may be a useful indicator of generalised stress as well as of infection.

Chemistry. Levels of the respiratory pigment haemocyanin can be measured by the haemolymph copper concentration. ${ }^{252}$ Routine measurements of the common inorganic ions (e.g. $\mathrm{Na}^{+}, \mathrm{K}^{+}, \mathrm{Ca}^{++}$, $\mathrm{Mg}^{++}, \mathrm{Cl}^{-}$and $\mathrm{SO}_{4}^{++}$) and protein ${ }^{253-257}$ would be helpful in establishing their utility as parameters for diagnosing disease. For example, in $O$. vulgaris a decrease in most inorganic elements and/or in haemocyte concentrations is observed when infection by the gastrointestinal parasite A. octopiana increases. ${ }^{258}$ Finally, measuring activity levels of respiratory enzymes and total protein concentration may provide additional information on the health status of an animal. ${ }^{259,260}$

Humoral agents. Plasma noradrenaline and dopamine increase transiently $(5 \mathrm{~min})$ in response to stress induced by air exposure in E. cirrhosa. ${ }^{255}$ However, it is difficult to envisage how such transient changes could be applied in routine health monitoring. Circulating levels of a number of hormones may give insights into health (e.g. steroids) and reproductive status, but normal ranges need to be established before these could be useful for health and welfare monitoring. Other molecules that may be of relevance for health monitoring include complement system molecules, anti-microbial peptides (AMPs) and other innate immunity-related proteins released by haemocytes, as they increase rapidly in infectious disease. ${ }^{261-266}$

e) Other biomarkers: analysis of faeces. One of the most promising methods to evaluate the physiological conditions of animals in laboratory settings is through the examination of faeces. Samples may be utilised to estimate various biomarkers including steroids (e.g. corticosteroids, estrogens, testosterone ${ }^{267}$ ), as well as to evaluate, for example, the digestibility of alternative diets as in the case of fish, ${ }^{267-270}$ or to identify possible parasite infections or cytological indicators of intestinal damage. ${ }^{271}$

We recommend the development of faecal analysis methods to assist in evaluation of health and welfare of cephalopods in laboratory settings.

\subsection{Health and welfare of ageing cephalopods: a special case?}

It is very difficult to determine the age of living cephalopods. Age is a parameter that is known for almost all other species used in research and should be included in the methods section of published papers (see ARRIVE Guidelines ${ }^{272}$ ), but which is rarely known in studies of cephalopods, unless they are laboratory reared.

Within a wild-caught population of a particular cephalopod species, in a circumscribed location and time of year, cephalopods of higher body weight are likely to be older, but the relationship between body weight and age is not linear, particularly in octopuses. ${ }^{238,273,274}$ The absence of precise age data complicates experimental design.

While there are variations due to the ecological niche of individual species, cephalopods generally live for about a year. With the exception of nautiloids, cephalopods undergo an exponential early growth phase during which they mature to adult size rapidly. However, this growth phase can be influenced by many factors, such as temperature, food availability and space, which makes body size a poor indicator of an animal's age. ${ }^{238,274-276}$ The age of sexual maturity is variable and also appears to depend on the ecological niche of the species. As reviewed by Rocha et al., some cephalopods (e.g. Loligo opalescens among squid and $O$. vulgaris among octopods) are semelparous (i.e. breed and then die soon after) while others (e.g. Nautilus sp., S. officinalis among cuttlefishes, L. vulgaris among squid and Octopus chierchiae among octopods) are iteroparous (i.e. breed multiple times, generally with longer lifespan). ${ }^{277}$ For a summary of reproductive strategies of some cephalopods species refer to Appendix 3.

In light of these considerations, ageing is relevant in the context of physical senescence (i.e. ageing changes in animals over time/after breeding, especially in females, once their eggs have hatched), but also when experimental procedures are applied to animals and age could influence the results.

Possible signs of cephalopods in senescence include reduced/absent drive to eat, poor skin quality, cloudy eyes, and changed activity pattern and behaviour. ${ }^{278-280}$ It may be difficult to distinguish this state from an animal that is showing similar signs due to disease. Good record keeping of time kept in the laboratory and age whenever possible, alongside general health records of individuals may help to differentiate the two situations.

It is unknown whether cephalopods experience any form of pain or suffering during senescence, but the precautionary principle should be applied when determining humane end-points (see section 8.3) for studies involving senescent cephalopods.

The senescent state makes animals more susceptible to a number of problems which, if they occurred in non-senescent animals, would be regarded as indicators of illness. ${ }^{145,278,280}$ These include: skin breaches 
including ulceration; primary and secondary cutaneous (e.g. Aeromonas sp., Vibrio sp. and Staphylococcus sp) and systemic (e.g. Flexobacter, Vibrio) bacterial or fungal (e.g. Labyrinthula sp., Cladosporium sp.) infections; increased parasite load. ${ }^{281,282}$

The senescent animals are not only more susceptible to infections, particularly of skin, ${ }^{283}$ but they also appear to have a reduced ability to recover once infected.

In general, animals showing signs of senescence should be humanely killed, unless there is sound scientific or animal welfare justification for keeping them alive.

A discussion of ethical aspects of both caring for and using senescent cephalopods in research is available in Smith et al. ${ }^{3}$

Careful routine monitoring of the physical condition of captive cephalopods at all life stages is essential for their proper care.

\subsection{Post-mortem evaluation}

Post-mortem evaluation of cephalopods is often a neglected aspect of health and welfare monitoring. It enables thorough inspection, revealing abnormalities not readily visible when the animal was living, the cause of death can be confirmed or ascertained, histological samples collected, and a database of findings can be gathered to support future post mortem evaluations.

The overall aim of such detailed evaluation is to facilitate better health and welfare assessments, and implementation of humane end-points, in future studies. Tissues such as the beak, statoliths and vestigial shells can also be collected, which may provide information on the age of the animal (for example for: O. vulgaris ${ }^{273,284}$ Sepioteuthis lessoniana, ${ }^{285,286}$ other cephalopod species ${ }^{287-300}$ ).

Cephalopod tissues are rich in protease enzymes, which cause rapid tissue autolysis post mortem. ${ }^{301,302}$ Autopsies should be performed immediately after humane killing an animal, for example, on welfare grounds when humane end-points have been reached, or at the end of a novel procedure and/or when the cause of welfare effects is uncertain; or as soon as an animal is found dead (see section 8.11), but only once death is confirmed (see section 8.12).

Cephalopods do not exhibit post mortem rigidity so rigor mortis cannot be used to confirm death, and other methods need to be employed (see section 8.12).

Autopsy findings should be reported in the first instance to the person responsible for overseeing the welfare and care of the animals, and any actions needed to safeguard animal welfare in future should be agreed, recorded and implemented.
Information on likely cause of death may be required for consideration by the local Animal Welfare Body or the National Competent Authority, especially if there is unexpected mortality following a procedure but note that mortality should never be used as an end-point for a procedure.

Steps to be considered for inclusion in post mortem evaluations include:

Haemolymph sampling: when animals are humanely killed for welfare reasons, it will be possible to collect haemolymph immediately surgical anaesthesia is achieved, but before death ensues (see section 8.5.5 for techniques). A bacterial septicaemia is suspected when the haemocytes have aggregated into visible clumps. Systemic bacterial infections should be confirmed by bacterial culture of the haemolymph.

Skin examination: external lesions should be blotted to remove excess mucus, then, aseptically, samples obtained using swabs and submitted for bacterial culture. Smears obtained from the swab or skin scrapings should be air dried and stained for bacteria or fungi.

Anatomical examination: descriptions of the gross internal anatomy of the main cephalopod species can be found in the following classic references: $N$. pompilius, ${ }^{303}$ S. officinalis; ${ }^{304}$ L. vulgaris; $;^{305}$ O. vulgaris; ${ }^{306} E$. cirrhosa. ${ }^{307}$

In brief, following a detailed external inspection including skin breaches, abnormal colouration, damage to appendages and deformities the mantle cavity is opened by an incision, following the anatomical approach that gives best accessibility in each species.

The viscera are examined visually and particular note taken of the state of the hearts, gills and the digestive gland (hepatopancreas), which is the largest and the main metabolic organ. Organs should be inspected for abnormal colour (particularly hepatopancreas), shape, size, texture (e.g. oedema, hard lump caused by a cyst or tumour), and presence of parasites (particularly intestine) or foreign bodies.

The presence, or not, of food (digested/ undigested) in the crop, stomach and caecum/intestine should be noted as well as faecal ropes in the rectum to assess gastrointestinal tract functionality. Digestive tract samples should be analysed for the presence of parasites.

The degree of filling of the ink sac should be noted as an empty one may indicate that the animal has inked profusely in the tank prior to death, which might not otherwise be apparent if the animal was found dead in a tank with circulating seawater.

Haemorrhage is impossible to detect as the blood is colourless when deoxygenated, and oedema may be hard to detect without histology.

Tissue samples can be fixed by immersion in neutralbuffered $10 \%$ formalin, and standard histo- 
pathological techniques applied although fixation in buffered glutaraldehyde will be required for ultrastructural studies and for identification of viruses.

Creation of a repository of data and/or reports on cephalopod pathology would provide an important resource in the effort to ensure good health and welfare in captive cephalopods used in laboratories. This is a current project of the non-profit Association for Cephalopod Research (see www.cephalopodre search.org/projects), which is also included as goal of the COST Action FA1301 (CephsInAction; http://www.cost.eu/COST_Actions/fa/Actions/

FA1301; www.cephsinaction.org).

\section{Disease: causes, prevention and treatment}

The major known risks to health and welfare in cephalopods are environmental parameters, and especially water quality issues, physical injury and infection (especially due to parasites), all of which may be interrelated. In addition, the psychological well-being of the animals should be considered (for a preliminary discussion see: ${ }^{308,309}$ ), as presented in section 6 .

\subsection{Introduction to the issues related to diseases of cephalopods}

7.1.1 Environmental influences on disease. Hostpathogen interactions can be strongly influenced by the environment in which an animal lives. In addition, stressful conditions deriving from inadequate physicochemical parameters (e.g. increased or decreased temperature, presence of undesirable chemicals, low oxygen saturation) may result in impaired defense responses against pathogens (review in ${ }^{310}$ ), thus increasing the probability of a disease outbreak.

Maintenance of water quality is essential for minimising infectious diseases and tank design (including enrichment) is important for avoiding self-induced physical trauma (e.g. 'butt-burn', see 6.1.2b above) and reducing general 'stress'.

Close attention to these factors should make animals more resistant to infection as well as improving psychological well-being.

7.1.2 Effects of physical trauma. Injuries may be inflicted by some methods of capture (e.g. tentacle loss by squid jig; ${ }^{311}$ see also ${ }^{312}$ ) and handling without appropriate care (e.g. skin damage by net reported $\mathrm{in}^{313}$ ), or during transport as, for example, for $O$. vulgaris that may fight if transported together and eventually bite or cannibalise each other (see description of fighting in $^{31}$ ).
Skin damage makes the animal susceptible to secondary infections (particularly bacterial) which can be fatal if untreated. ${ }^{314}$

7.1.3 Disease caused by feeding live food. Although feeding with live food may be preferred to an artificial diet (see section 4.2), it is important to avoid the use of species that are recognised for their role as hosts of important pathogenetic parasites. For example, coccidian Aggregata sp. ${ }^{258,315,316}$ (for review see ${ }^{73}$ ) or viruses ${ }^{317-319}$ may infect cephalopods through food items such as crustaceans. In the case of the use of crabs as a prey item, special attention should be given to distinguish between those carrying parasites and to remove them from cephalopod facilities.

It is also noteworthy that penetrative injury to the brain has been reported to occur in O. vulgaris ${ }^{320}$ due to the setae of ingested polychaete scaleworms (Herminone hystrix) so their presence in laboratory tanks should also be avoided.

See also section 7.2 below for discussion on infectious diseases of cephalopods.

\subsubsection{Action points when disease is} suspected. Animals showing signs of illness should be placed in quarantine (see section 3.6) to reduce the potential spread of the causal agent, and any animals found dead removed and autopsied (see section 6.3). Water from tanks of quarantined animals should not contaminate water supplies to other animals or the environment, and personnel handling potentially infectious animals should wear protective clothing (see section 9). Seriously ill animals not responding to treatment (where treatment is possible) should be humanely killed (see section 8.11) and autopsied immediately (see section 6.3).

Investigation of disease outbreaks should not only include identification of the immediate cause (e.g. infectious agent), but also underlying origin such as adverse water quality, contamination of food or effects caused by other animals. Failure to correct such factors will often result in further outbreak of disease.

Careful records must be kept of all occurrences of illness or mortality irrespective of identification of cause, so that patterns can be identified over time (e.g. there might be higher mortality/infection rates from certain suppliers).

\subsection{Infectious agents in cephalopods}

Immunity in cephalopods differs from vertebrates due to the absence of an adaptive immune response. ${ }^{321,322}$ However, these animals do have an innate (nonspecific) immune response, mediated by both humoral (e.g. haemagglutinin) and cellular (haemocyte) mechanisms. ${ }^{73,252,323-325}$ 
As in other molluscs, circulating haemocytes are responsible for infiltration, aggregation, encapsulation, cytotoxic reactions and phagocytosis of foreign particles. Cowden and Curtis estimated that the phagocytic capacity of octopus haemocytes was low; ${ }^{326}$ while high phagocytosis of carbon particles has been described in E. cirrhosa. ${ }^{327}$ Phagocytic capacities of the haemocytes of the common octopus, O. vulgaris, challenged in vitro using zymosan as a test particle, ${ }^{325}$ and those of the haemocytes of $E$. dofleini (see citations in ${ }^{328}$ ) have been reported. Recently, an extensive analysis of octopus haemocytes at morphological, flow cytometry and functional level (including phagocytic capability as well as reactive oxygen species (ROS) and nitric oxide production) after challenging with different stimuli has has been carried out by C. Gestal and coworkers. ${ }^{73,329}$ In addition, several biologically active molecules likely to be involved in responses to infection and injury are known to be present in the haemolymph of cephalopods, such as lectins, proteinases, including antiprotease and lysozyme activities. ${ }^{126,266,330-332}$

The immunobiological system in cephalopods is quite effective, as reflected by the scarce reporting of illness in captivity for this class over many years, but this low incidence could also reflect under-diagnosis, particularly of systemic disease that may not have an external manifestation, or under-reporting.

7.2.1 Viruses. Viruses are the most abundant component of aquatic microbial communities (for review refer to $^{333-335}$ ). However, there are few records of virus-inducing pathologies in cephalopods as reviewed in Hanlon and Forsythe. ${ }^{336}$ The first known evidence of viral infections in cephalopods was provided in specimens of $O$. vulgaris and in the cuttlefish S. officinalis. ${ }^{337,338}$ In the octopus, oedematous, modular tumors embedded in arm musculature and tissue degeneration were observed in animals showing anorexia, apathy and often autophagy. ${ }^{337,339}$ The lesions were linked to the presence of viral particles suggested to belong to the group of iridovirus, according to their size, morphology and location. ${ }^{340}$ In $S$. officinalis, virus-like particles were identified in the gastric epithelium and described as similar to reoviruses of vertebrates, but details on the symptoms induced are not provided. ${ }^{338}$ Virus-like particles have been also reported in the epithelial cells of the tubules of the digestive gland of Loligo pealei, and in the renal appendages of several octopod species. ${ }^{336}$

More recently, Gregory and coworkers ${ }^{341}$ reported another possible infection of iridovirus in cephalopods (i.e. Nautilus sp.). Intracytoplasmic inclusion bodies were observed in tissues from an animal found dead in aquarium without premonitory signs of disease.

Furthermore, Todarodes pacificus (utilised as a food item in aquaculture) have been reported to be positive for Betanodavirus, ${ }^{342}$ which is the aetiologic agent of a serious viral disease known as VER (viral encephalopathy and retinopathy) that has been detected in a wide range of vertebrate and invertebrate hosts worldwide and caused severe mass mortalities in both farmed and wild marine organisms. ${ }^{343}$ Betanodavirus was also identified in skin lesions, in the eye and in the branchial heart of $O$. vulgaris. ${ }^{344}$ Squid have been suggested also as possible vectors of zoonotic viral agents such as Norovirus. ${ }^{345-347}$

Infections from viruses may sometimes be asymptomatic (see examples from fishes: ${ }^{348,349}$ ) but pathogenicity may be higher if temperature increase, as in the case of global environmental changes. ${ }^{350} \mathrm{In}$ fishes, symptoms of viral infection may include clouding of the eye, anorexia, changes in body colour and uncoordinated swimming.

7.2.2 Bacteria. In cephalopods, pathogenic bacterial infections are caused by several microbes; for an overview see Table 6 . These include various species of Gram-negative Vibrio (review in ${ }^{336,351}$ ). However, Vibrio bacteria can also be symbiotic, as for the case of the Hawaiian bobtail squid (E. scolopes) where Vibrio fischeri is a mutualist in the light organ, ${ }^{352,353}$ as well as Psedomonas sp. and other bacteria that are symbionts in Nautilus sp. ${ }^{354}$

Secondary bacterial infections in skin lesions have been reported in squid, ${ }^{355}$ cuttlefish $^{356}$ and octopus, ${ }^{357}$ and skin lesions are considered to be the most common conditions in which infections occur. ${ }^{351}$ Bacterial infections may spread to conspecifics sharing the tank. ${ }^{77}$ In addition, bacteria may cause skin ulcers on mantle, head and arms, hyperplasia of the epidermis and increased mucus production (e.g. in Lolliguncula brevis $;{ }^{355}$ in $O$. joubini and O. briareus ${ }^{357}$ for review $\left.\sec ^{336,358}\right)$.

While infections occurring on the skin are most commonly reported, they are not the only tissues susceptible to bacterial infection, since Rickettsiales-like organisms have been found in the gills of laboratory reared $O$. vulgaris, observed as basophilic intracytoplasmatic microcolonies within epithelial cells, on which they cause hypertrophia and occasionally necrosis. No significant harm has been observed in the host, but under conditions of stress or intensive husbandry, it has been suggested that these bacteria may have a detrimental effect on the host's respiratory gaseous exchange although this has not been shown experimentally. ${ }^{359}$

Gram-negative bacteria Vibrio lentus have been also identified in the branchial heart of wild $O$. vulgaris and reported to induce mortality in $50 \%$ of octopuses in the first six hours, with lesions showing a typical round pattern on the arms or head. ${ }^{360}$ 


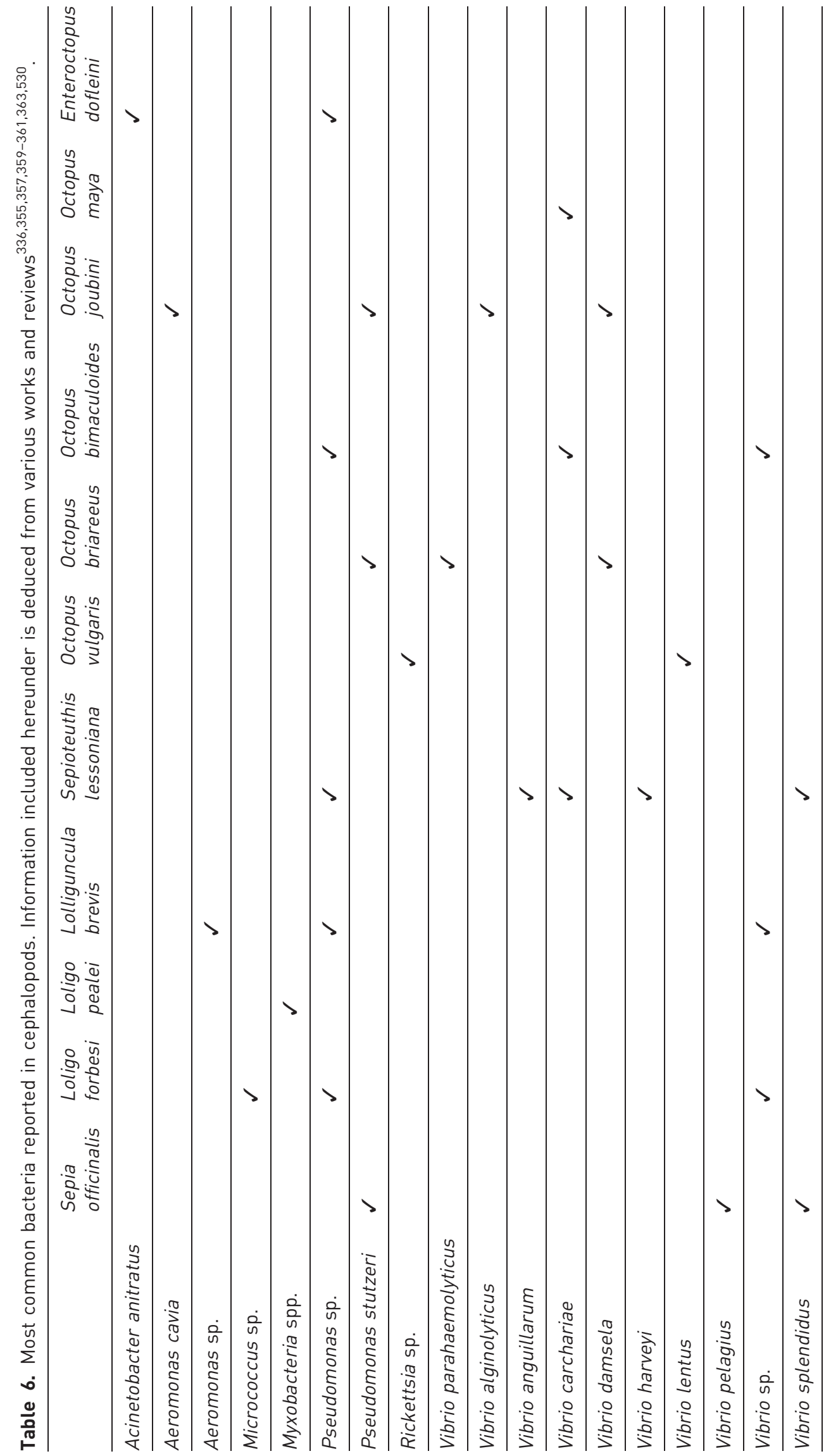


Finally, cloudy-to-opaque corneal tissue as well as opaque lenses in Loligo forbesi and S. lessoniana have been reported due to infection with Gram-positive bacteria (Microccocus sp.) found in the vitreous-induced swelling of the infected eye and causing opacity of the cornea. ${ }^{361}$

7.2.3 Fungi. Reports of fungal infections in cephalopods are scarce and mostly relate to eggs and embryos. Hanlon and Forsythe ${ }^{336}$ refer to infection by Labyrinthula sp. in adult $O$. vulgaris; in these animals grey patches of inactivated chromatophores appeared followed by progressively larger and whiter patches in which the entire epidermis and dermis was missing. Thraustochytrid and labyrinthulid fungi have also been isolated from skin lesions in E. cirrhosa, but it is not clear whether these organisms are causal agents or secondary infections. ${ }^{362}$

Harms et al. ${ }^{282}$ reported a case of mycotic infection in adult captive cuttlefish showing skin lesions in the dorsal mantle. Cytology revealed hemocyte granulomas surrounding fungal hyphae, and culture yelded Cladosporium sp. Infection from the same organism was also reported by Scimeca and Oestmann (1985, cited $\mathrm{in}^{351}$ ) in octopus, while Fusarium sp. has been found infecting the chambered nautilus, $N$. pompilius. ${ }^{351}$

7.2.4 Parasites. Most wild cephalopods host parasites include protozoans, dicyemids and metazoans. Generally, these are found in skin, gills, digestive tract, digestive gland and kidneys. ${ }^{73,363-370}$

Among the protozoans, one of the main parasites infecting both wild and cultured cephalopods is the gastrointestinal coccidian of the genus Aggregata, which produces severe disease in cuttlefishes and octopuses, by causing a malabsorption syndrome, diminishing nutrient absorption and reducing the immune response capability. ${ }^{226,258}$ In addition, the parasite may produce behavioural alterations in the infected host inducing excitation, impaired ability to camouflage and aggressive behaviour. ${ }^{316}$ Mortality has been attributed to the infection, and it has been reported that a few days before dying an infected octopus became inactive in the shelters and unresponsive to stimuli. ${ }^{316}$

Dicyemids are endosymbionts that inhabit the renal sacs of cephalopods including cuttlefish, loliginid squid and octopuses. ${ }^{370-372}$ No host damage has been reported due to dicyemids, but a possible contribution to ammonium ion elimination from the host urine has been proposed ${ }^{370}$. However, dicyemids could be a problem if the parasite load is elevated enough to cause physical obstruction of the renal sac.

Cephalopods are intermediate or parathenic hosts for a variety of metazoan parasites, namely trematodes, digenea, cestodes and nematodes transmitted via the food chain. ${ }^{370,373}$
Amongst these, one of the most abundant and frequent parasites are anisakid nematodes, which have been reported to cause important pathological effects in several cephalopod host species. ${ }^{374-376}$ Larvae of Anisakis simplex are pathogenic to humans when raw, under-cooked or lightly marinated fish or squid are ingested. ${ }^{377}$ Crustaceans, such as copepods and isopods, also parasitise the gills and mantle cavity of cephalopods, affecting the body condition of the host. ${ }^{378}$

Apart from the potentially pathogenic organisms (e.g. Vibrio, Aeromonas, Pseudomonas and Flavobacterium sp.; ciliates and dicyemids), none of these diverse organisms is known to cause severe health problems in captive cephalopods. However, as some of the mechanisms exploited by parasites to produce changes in host's behaviour would seem to be highly conserved throughout the evolution of both vertebrates and invertebrates, ${ }^{379-382}$ it would be unwise to exclude such interactions in cephalopods without specific research.

\subsection{Antibiotic treatment of infectious diseases}

Antibiotics have been utilised in some instances to treat cephalopods in laboratory experiments as reviewed in Table 7. Several routes of administration have been used (i.e. oral, parenteral or tank/bath immersion); in addition Berk and coworkers ${ }^{383}$ have suggested a technique for gavage in squid that could be adapted to octopus and cuttlefish. Intramuscular injections of antibiotics have been given at the base of the arms taking special care to avoid the axial nerve cord. ${ }^{358}$ Sherrill et al. ${ }^{356}$ have suggested the use of oral, parenteral, or tank/bath immersion prophylactic antibiotics as reasonable for captive cuttlefish subjected to physiological stress, since this treatment may delay disease progression and improve longevity. This method should be avoided unless there are exceptional, scientifically justified circumstances, as it is clearly preferable to identify and remove the source of the stress.

Despite published evidence, caution should be applied when using oral or parental routes of administration since these are stressful for animals and may be difficult to perform safely.

In any case, prophylactic use of antibiotics is not recommended because of the risk of promoting bacterial and fungal resistance, masking infection and allowing secondary infection. It should not be used to 'prop up' poor tank hygiene.

Cephalopods used for scientific purposes should be maintained free from infections and contact with infection sources avoided. To this end, the use of high-performing filtration systems (i.e. combining mechanical, biological and physical filtrations) is highly recommended in combination with careful 
Table 7. Substances given to some cephalopods to treat infection, but not recommended for routine use in laboratory facilities; see text for details. The table summarises for each species the treatment (i.e. dosage, route and duration) and substances tested. All drugs included in this table belong to the class of pharmaceuticals utilised as antibacterial agents, unless otherwise stated. The studies cited refer to treatment of infections, with the exception of Gore et al. ${ }^{530}$ who

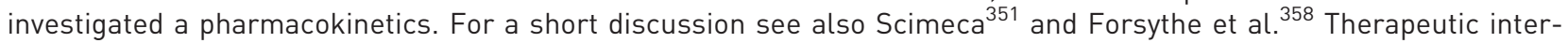
ventions should be discussed with a veterinarian.

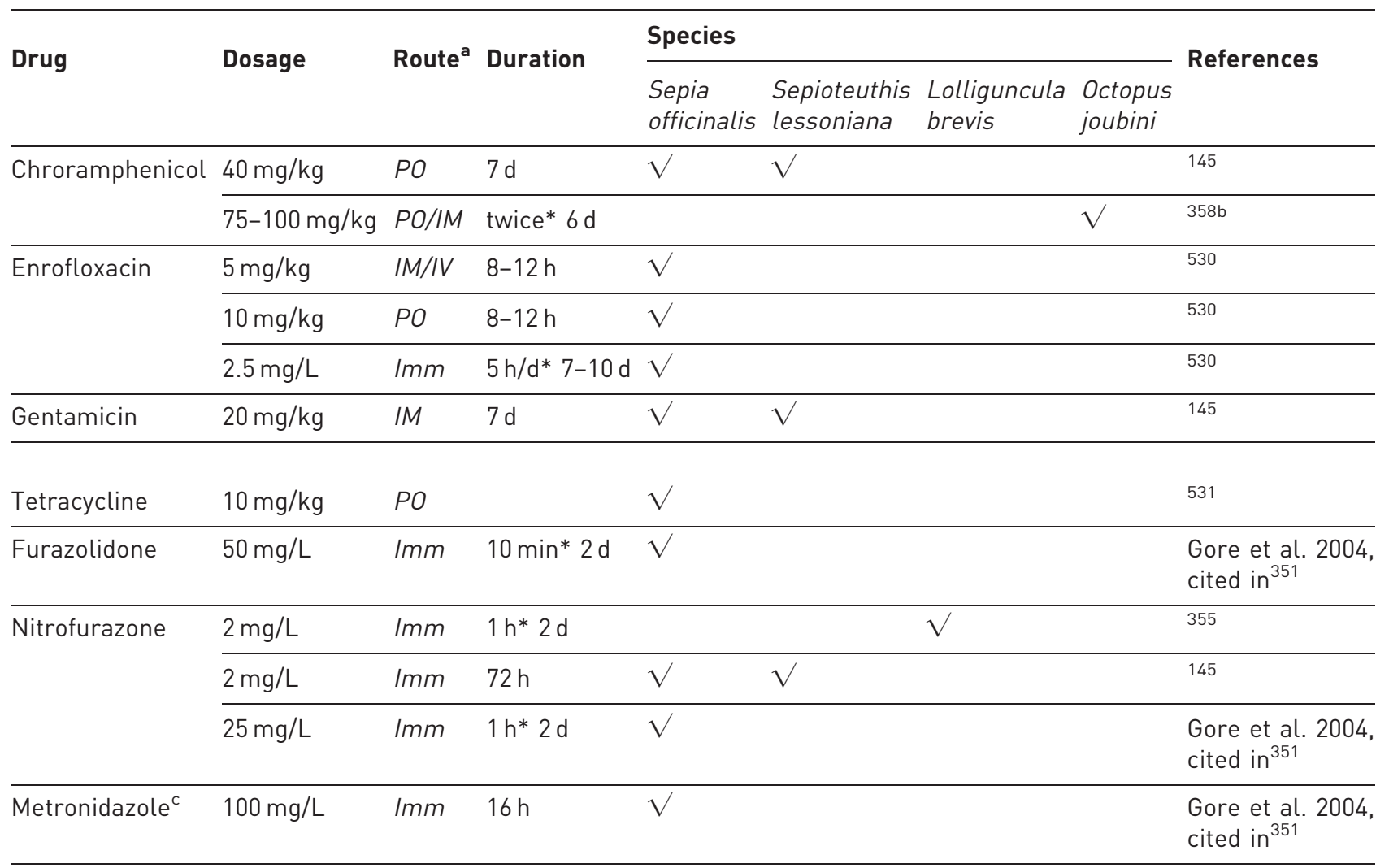

${ }^{a}$ Administration route: $P O$, per os (i.e. provided through food items); $I m m$, immersion in a solution; $I M$, intramuscular injection; IV, intravenous injection.

${ }^{\mathrm{b}}$ But see also Table 1-3 in Hanlon and Forsythe. ${ }^{336}$

${ }^{c}$ Antiprotozoal agent.

screening of animals entering the facility and efficient quarantine procedures. The importance of tank design in minimising the potential for skin damage and subsequent increased probability of infection should not be overlooked.

\section{Scientific procedures, severity and harm-benefit assessment, anaesthesia and humane killing}

\subsection{Definition of a 'procedure'}

Directive 2010/63/EU defines a regulated 'procedure' as, Any use, invasive or non-invasive, of an animal [e.g. living cephalopod] for experimental or other scientific purposes, with known or unknown outcome, or education purposes, which may cause the animal a level of pain, suffering or lasting harm equivalent to, or higher than, that caused by the introduction of a needle in accordance with good veterinary practice'.

It should be noted that this definition is not confined to procedures that induce pain, but also includes procedures that cause other forms of suffering, such as anxiety, fear, stress and distress. Table 8 illustrates this point by listing some studies which include procedures that are likely to be subject to regulation under the Directive.

\subsection{Identifying and reducing the adverse effects of procedures}

All adverse effects that could be caused to animals by particular scientific procedures must be identified and predicted at the project planning stage (prospective assessment), then adequately monitored throughout 
Table 8. Selected examples of research in cephalopods which involves an intervention that it is considered would come within the definition of a procedure (see also section 8 of this work) within the Directive. Studies published recently have been selected where possible to show that the Directive will impact on current research areas. It should be noted that non-surgical interventions that may induce PSDLH fall within the definition of a procedure. The fact that a particular technique has been used in a previously published study does not guarantee that the same technique would now be permitted by the national competent authority under the Directive.

\begin{tabular}{|c|c|}
\hline Research topic or technique and species studied & References \\
\hline $\begin{array}{l}\text { Amputation of a portion of an arm under general anaesthesia followed by recovery } \\
\text { Octopus vulgaris, Sepia officinalis, S.pharaonis, Doryteuthis pealeii }\end{array}$ & $10,190,431$ \\
\hline $\begin{array}{l}\text { Deprivation of 'sleep' for } 48 \mathrm{~h} \text { by continuous visual stimulation } \\
\text { Sepia officinalis }\end{array}$ & 102 \\
\hline $\begin{array}{l}\text { Administration of } E \text {. coli lipopolysaccharide by intramuscular injection into the arm under } \\
\text { general anaesthesia followed by recovery and subsequent repeated sampling of haemo- } \\
\text { lymph } \\
\text { Octopus vulgaris }\end{array}$ & 251 \\
\hline $\begin{array}{l}\text { Investigation of the efficacy of different general anaesthetic substances techniques and } \\
\text { mechanisms of anaesthesia } \\
\text { Sepia officinalis }\end{array}$ & 440 \\
\hline $\begin{array}{l}\text { Production of hatchlings with deleterious phenotypes/genotypes by exposure of the eggs } \\
\text { to: a harmful environment, or mutagen, or genetic manipulation } \\
\text { Loligo vulgaris }\end{array}$ & 532 \\
\hline $\begin{array}{l}\text { Implantation of electrodes for either recording or stimulation into the brain under anaes- } \\
\text { thesia followed by investigation of the effects in the conscious/sedated animal } \\
\text { Octopus vulgaris, Doryteuthis pealeii }\end{array}$ & 208,533 \\
\hline $\begin{array}{l}\text { Removal of samples of haemoloymph from the dorsal aorta under anaesthesia with recov- } \\
\text { ery } \\
\text { Euprymna scolopes }\end{array}$ & 249 \\
\hline $\begin{array}{l}\text { Administration of drugs to modify nervous system functionality } \\
\text { Doryteuthis pealeii, Sepia officinalis }\end{array}$ & 396,402 \\
\hline $\begin{array}{l}\text { Implantation of temperature and depth-logging archival tags under general anaesthesia } \\
\text { with recovery and monitoring for up to } 5 \text { months } \\
\text { Sepia officinalis }\end{array}$ & 130,132 \\
\hline $\begin{array}{l}\text { Non-invasive measurement of brain and arm morphology under anaesthesia with recovery } \\
\text { Octopus vulgaris }\end{array}$ & 246,247 \\
\hline $\begin{array}{l}\text { Immobilisation of animal and exposure to light stimuli to investigate the pupillary reflex } \\
\text { Lolliguncula brevis }\end{array}$ & 215 \\
\hline $\begin{array}{l}\text { Food deprivation for } 7 \text { or more days } \\
\text { Sepia officinalis }\end{array}$ & 534,535 \\
\hline $\begin{array}{l}\text { Aversive training paradigms to test acquisition, consolidation and memory recall } \\
\text { Sepia officinalis, Octopus vulgaris }\end{array}$ & $26,420,504,506,507$ \\
\hline
\end{tabular}

the procedure. Steps must be taken to: i. refine each procedure, so as to minimise and preferably eliminate its adverse effects, and ii. alleviate any animal suffering that occurs during the conduct of procedures or whilst animals are recovering.

This is a legal, as well as ethical, requirement under Directive 2010/63/EU, which requires implementation of replacement, reduction and refinement (3RS) strategies (section 2) wherever possible, with the aim of 'eliminating or reducing to a minimum any possible pain, suffering, distress or lasting harm' [PSDLH] caused to the animals. Note that $3 \mathrm{Rs}$ strategies should be implemented whenever feasible, at all times from birth to death of the animals including: sourcing, transport, housing and care, handling, and fate of animals, as well as the procedures themselves. 


\subsection{Monitoring animals undergoing procedures and setting humane end-points}

Assessment of the severity of adverse effects must be carried out before, during and after procedures (see also details in section 2.2 above, which describes requirements for setting prospective 'severity limits' and retrospective reporting of the severity of procedures, along with questions for consideration).

Schemes for monitoring adverse effects during the procedure should cover the criteria outlined in section 6 and Table 5 for routine daily assessment of animal welfare; these should be supplemented with any other, specific, adverse effects that might be caused by the particular procedure(s). Criteria for assessment, and frequency and timing of observations, should be agreed before studies commence; then regularly reviewed as studies progress, and, wherever necessary, added to or amended.

A humane end-point must also be defined for each procedure, to describe (in terms of indicators of the nature and degree of suffering) the earliest point at which a specific intervention must be made to end an animal's suffering, e.g. by: $i$. removing the animal from the study, $i i$. providing analgesia, iii. humanely killing the animal and/or terminating the study. The use of 'score sheets' for monitoring can be particularly helpful in determining when humane end-points have been reached, and when severity limits are being approached. ${ }^{384}$ To be effective, this monitoring requires a team approach, with good planning, and appropriate training for all involved. Methods for the observation and assessment of adverse effects are relatively well developed for many vertebrate species including fish. ${ }^{384-387}$

There is a need for further development of objective criteria for assessing severity that can be used by the entire EU cephalopod community to ensure consistency. This is a current project of the non-profit Association for Cephalopod Research (see www.cepha lopodresearch.org/projects).

Working on a consensus view for severity assessment of procedures is also a goal of the FA1301 COST Action (CephsInAction; http://www.cost.eu/COST_Actions/fa/ Actions/FA1301; www.cephsinaction.org). An equivalent initiative has been set up for describing characteristics of laboratory mice (http://www.mousewelfareterms.org/ doku.php), ${ }^{8}$ and and for severity classification classification of scientific procedures involving fish. ${ }^{384}$

\subsection{Harm-benefit assessment}

Of course, one way of eliminating animal suffering is not to carry out the procedure at all. In this context, it must be remembered that procedures may only be carried out within the framework of a [authorised] project' (Directive, Article 12.2), which is subject to a harm-benefit analysis, 'to assess whether the harm to the animals in terms of suffering, pain and distress is justified by the expected outcome taking into account ethical considerations, and may ultimately benefit human beings, animals or the environment'.

Hence, for legal as well as moral reasons, investigators should carry out a harm-benefit analysis, as described above, prior to conducting any procedure.

In the following discussion, we cite published studies that provide evidence about possible harms caused by common procedures. These are included as examples that will help to predict and identify harms in future projects, but we are not suggesting that these studies would necessarily be considered justified according to the harm-benefit analysis conducted under the new EU Directive.

\subsection{Some common procedures in cephalopod research}

The following sections summarise current knowledge of the regulatory status, adverse effects and possibilities for refinement of some common procedures. It is evident that this knowledge is patchy and there is need for further work, especially to help refine procedures. Nevertheless, where possible, provisional recommendations for good practice are made.

8.5.1 Behavioural studies. Cephalopods have been used extensively for a variety of behavioural studies, as reviewed in several works. $2,25,36,175,192,195,388$

Classical behavioural studies have used a variety of aversive stimuli (e.g. electric shock; ${ }^{72}$ acid solutions; ${ }^{389}$ bitter, ${ }^{390}$ mechanical ${ }^{391}$ ) as part of training protocols. Because of their potential to cause distress and possible suffering, such studies would certainly fall within the definition of a regulated procedure under the Directive and should be avoided wherever possible. For example, electric shock or application of acid solutions should be unacceptable, and these and other harmful stimuli should be replaced with reward-based conditioning and, at the least, stimuli that do not cause pain.

Exposure of an animal to a stimulus known to evoke an escape response (i.e. inking, jetting locomotion, dymantic display) could also be argued to cause distress, especially if the exposure is repeated, and hence is likely to be a regulated procedure. Deprivation of food for prolonged periods, deliberate exposure to elevated noise or adverse change in water temperature, $\mathrm{pH}$ or chemistry, and direct exposure to a predator would also be likely to fall within the definition of a procedure, depending on the severity/degree of change. 


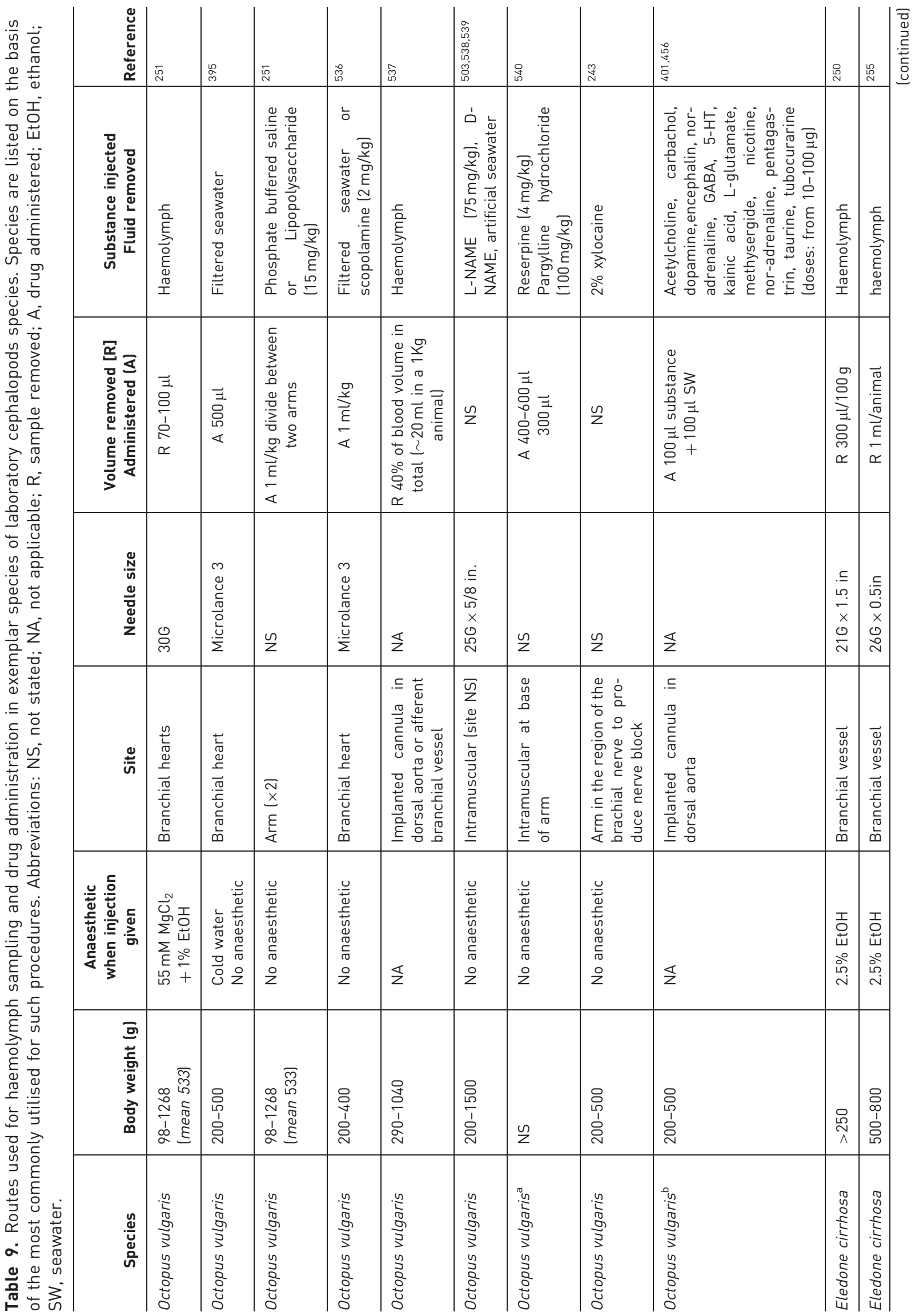




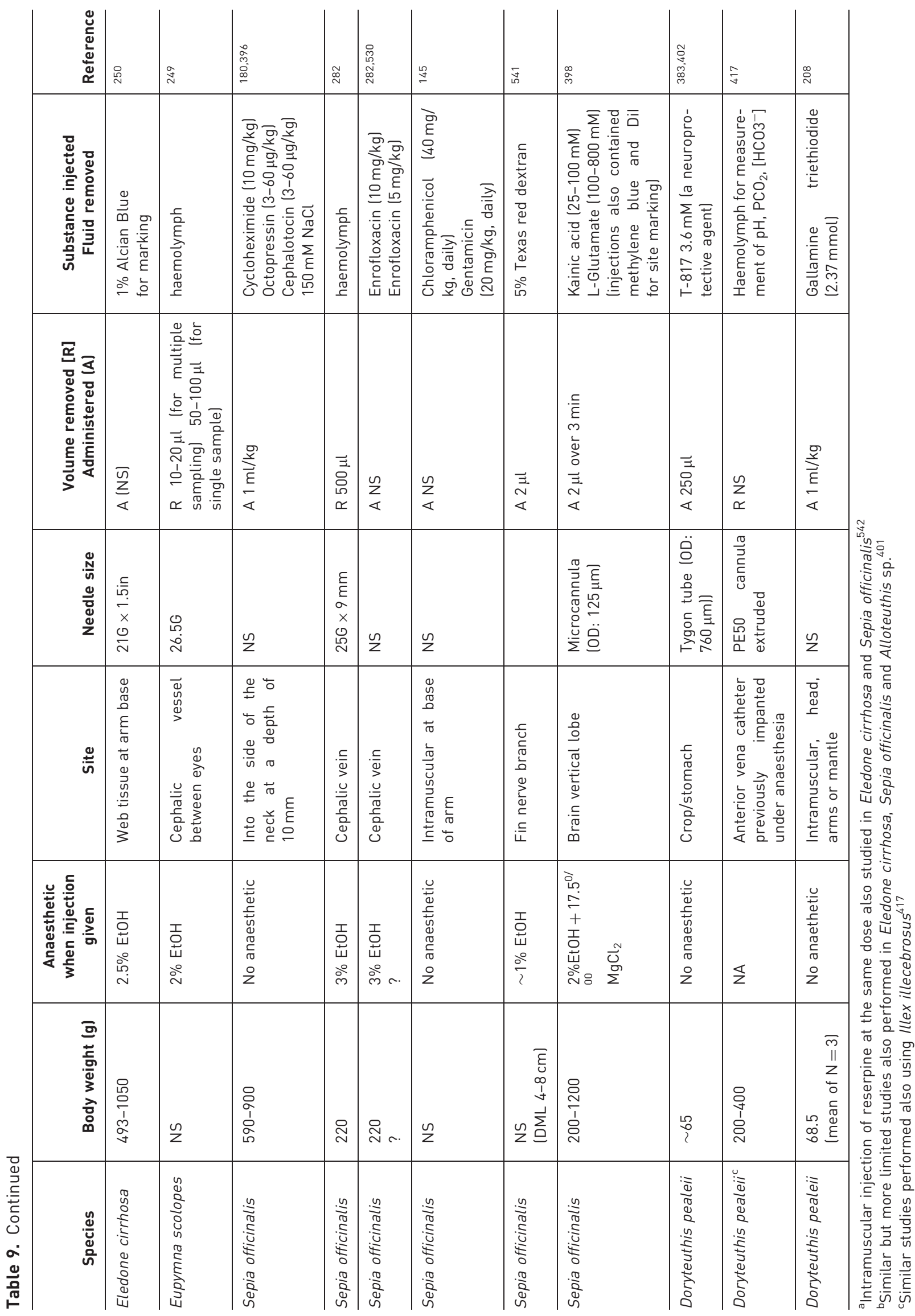


Protocols using positive reinforcement (for example see: ${ }^{104,392-394}$ ) should be used wherever possible. ${ }^{2}$

8.5.2 Administration of substances. Administration of substances can cause harms to animals as a result of the route of administration and/or the substance itself. Table 9 lists commonly-used routes of administration, but does not recommend a particular technique since, despite the number of examples included, there have been no systematic studies investigating the optimal size of needles or injection sites and volumes in cephalopods. In addition, although adverse effects of injections have not been reported, in view of the limited literature this needs further investigation from a welfare perspective. $^{251,395}$

Cephalopods do not have readily accessible large superficial blood vessels; therefore, in general experimental agents and therapeutic drugs are given to unanaesthetised animals by bath application (assuming absorption via skin and gills), or injection via subcutaneous and intramuscular routes.

The efficacy of subcutaneous injections is not known, but drug absorption may be slow because of a low capillary density. However, this is not based on pharmacokinetic studies and only a limited number of substances have been studied. ${ }^{123,395}$

Locations for intramuscular injections include the arms (particularly proximal parts in octopus) and 'neck' (in cuttlefish) reported to have a high vascular density. ${ }^{123,180,208,251,396}$ Care should be taken not to damage arm nerve cords and ganglia by injection in the arms (especially in octopus), nor the cuttlebone or gladius in cuttlefish and squid respectively. The use of ultrasound may be helpful in directing injections to avoid vulnerable structures. ${ }^{246,247}$

Substances have been injected into the branchial hearts of $O$. vulgaris, but this requires eversion of the mantle. The animals recover rapidly from this procedure and no adverse events have been reported; however, although behaviour rapidly $(<1$ hour) returned to normal, ${ }^{395}$ a systematic study of welfare of the animal has not been fully carried out.

8.5.3 Other methods. It should be noted that the welfare effects of the following methods has not been assessed. Intravenous (including vena cava) and intradorsal aortic routes have been used for substance administration by direct injection.e.g. ${ }^{282}$ Direct administration into the brain has been successfully attempted in a number of studies. e.g. ${ }^{397,398}$. Finally, surgical implantation of vascular catheters has been used as a method of drug administration and for pressure recording. ${ }^{399-401}$

A method for gavage administration of drugs to cephalopods has been described by Berk et al. ${ }^{383}$ and used to investigate the efficacy of a putative neuroprotective agent in blocking the effects of human tau protein on transmission in the squid giant synapse. ${ }^{402}$ This method requires restraint of the animal and may require removal from the water and sedation. As the brain in cephalopods encircles the oesophagus care should be taken to use the narrowest gauge tube as possible, and it should also have a round end to minimise the chances of puncturing the gut particularly at the oesophageal/crop junction. Substances administered by this route will reach the crop and stomach directly.

Drugs and other substances such as nanoparticles have also been administered per os (i.e. by inclusion in the food, ${ }^{403}$ G. Ponte, unpublished data). Further experiments may help in better refine this approach.

8.5.4 Administration of drugs as investigational agents. When undertaking studies using pharmacological agents, the wider effects of the drug must also be considered in assessing the overall impact of a treatment on the welfare of the animal, and any unexpected side-effects should be recorded and minimised/avoided where possible. For example, Agin et al. ${ }^{180}$ used cycloheximide, a protein synthesis inhibitor, to investigate the machinery involved in long-term memory in the cuttlefish. However, it induced positive buoyancy that interfered with animals' ability to catch prey.

Relatively little is known about the pharmacology of central and peripheral neurotransmitter systems. ${ }^{1,404,405}$ In addition, very limited is the knowledge on the pharmacological characteristics of drug receptors in cephalopods. Therefore, caution should always be exercised when drugs, whose pharmacological properties have been defined in mammalian systems, are used as investigational agents in cephalopods.

If such studies are undertaken in group housed species, it will be necessary to identify individual animals (see section 4.1.2) so that individual variations in drug response can be identified. Additionally in grouphoused species, special attention should be paid to agents that may increase aggression (e.g. by alteration of brain neurotransmitters) or that may impair the ability of an individual to escape or defend itself and action taken to reduce or eliminate these adverse effects.

8.5.5 Haemolymph (blood) collection. It should be noted that cephalopod blood does not clot, but haemocytes aggregate and vessels constrict to prevent blood loss. ${ }^{150,406}$ However, as haemolymph is pale blue (oxygenated) or colourless (deoxygenated), haemorrhage may be very difficult to detect.

A summary of routes used in several studies for haemolymph sampling is presented in Table 9. There are no systematic data on the welfare impacts of blood sampling methods in cephalopods, but in several 
studies behaviour was reported to return to normal following the sampling. ${ }^{249,251,395}$

In large cephalopods (i.e. E. dofleini), cuttlefish and E. scolopes, small blood samples have been obtained under anaesthesia via a needle inserted into the cephalic vein dorsal to the funnel. ${ }^{249,282,407,408}$ This method has also been successfully applied for haemolymph sampling in O. vulgaris (G. Ponte and G. Fiorito, pers comm.). In addition, small single samples of haemolymph can be taken directly from the branchial hearts in $O$. vulgaris $(\sim 250 \mu \mathrm{l})$ and E. cirrhosa $(<300 \mu \mathrm{l} /$ $100 \mathrm{~g}$ body weight; $1 \mathrm{ml} /$ animal in animals of $500-800 \mathrm{~g}$, as shown $\mathrm{in}^{250}$ ) using a hypodermic needle, although this requires general anaesthesia to permit manipulation of the mantle to expose the hearts. ${ }^{250,251,255,403} \mathrm{In}$ the bobtail squid (E. scolopes) single haemolymph samples of $50-100 \mu \mathrm{l}$ can be withdrawn from the dorsal aorta by direct needle puncture under anaesthesia. ${ }^{249}$ Where frequent sampling of blood is necessary, it is likely that this will require implantation of catheters. ${ }^{240,407,408}$ Again, the welfare impact of this procedure has not been fully assessed.

The maximum volume of blood which can be collected at a single sampling in particular cephalopods has not been assessed from a welfare perspective. In mammals, it is recommended that no more than $10 \%$ of the blood volume is removed at any one time. ${ }^{409}$ For fish the Canadian Council on Animal Care (CCAC) recommends a maximum withdrawal of $1 \mathrm{ml}$ blood $/ \mathrm{kg}$ weight.

Blood volume in octopus is estimated at $5-6 \%$ of body weight, ${ }^{410,411}$ hence using the mammalian values as a guide, a maximum of $5-6 \mathrm{ml} \mathrm{blood} / \mathrm{kg}$ weight would be advised, but, as noted above, only $1 \mathrm{ml} / \mathrm{kg}$ according to CCAC guidelines for fish.

However, in publications where multiple sampling has been used, authors either collected a reduced volume on each occasion (e.g. 10-20 $\mu$ for multiple sampling vs 50-100 $\mu$ for single samples in bobtail squid) as done by Collins and Nyholm ${ }^{249}$ or have set a time for recovery between samples (e.g. 4 hours in E. cirrhosa). ${ }^{126}$

\subsection{Surgery}

Cephalopods have been subjected to a broadly similar range of surgical procedures as have been performed in vertebrates, but in general surgical techniques are not described in detail in publications; this is no longer considered acceptable and we strongly recommend following ARRIVE Guidelines on reporting. ${ }^{272}$

Surgical approaches and techniques, along with understanding of their welfare effects, are not as

\footnotetext{
see: http://www.ccac.ca/Documents/Education/DFO/4_Blood_ Sampling_of_Finfish.pdf
}

advanced in cephalopods when compared with vertebrates, particularly in relation to: $i$. intra-operative monitoring and maintenance of physiological parameters (e.g. blood pressure, $\mathrm{PO}_{2}, \mathrm{PCO}_{2}, \mathrm{pH}$, temperature; see also section 8.8.4); ii. identification and control of haemorrhage; iii. controlled general anaesthesia and analgesia (see section 8.8); iv. optimal techniques for the repair of muscle and skin tissue and wound closure (see section 8.6.2) and healing in general; v. post-operative monitoring and special care that may be needed if, for example, feeding is transiently impaired; vi. infection risk (intra- and post-operatively) and requirement for prophylactic antibiotic cover (see section 7.3).

Although it will be some time before there is welfare guidance on all the above topics, anyone contemplating undertaking a surgical procedure in cephalopods will need to consider all of the factors, and submit relevant information, as part of the project evaluation and authorisation process, and during the conduct of any authorised surgical procedures. This will include:

(i) ethical 'justification' for the procedures, in terms of harm vs. benefit of the outcomes;

(ii) identification of potential adverse effects and the steps taken to refine the procedures, so as to minimise the adverse effects;

(iii) processes for intra- and post-operative monitoring of the animals;

(iv) timely application of methods to minimise postoperative suffering, e.g. analgesia and other specific care; and

(v) clearly defined humane end-points (section 8.3) to set an upper limit to the suffering that an animal experiences.

Some general principles for performing surgery on aquatic animals can be found in the fish literature, and these should be adapted where possible to cephalopods. ${ }^{412-414}$

As for all other procedures, anyone attempting any surgery in cephalopods must be 'adequately educated and trained' in the principles and practical techniques of surgery, and must be 'supervised in the performance of their tasks, until they have demonstrated the requisite competence' (Directive Article 23\$2), for example, to the designated veterinarian or other competent person (see section 10 for further discussion).

A full description and evaluation of surgical techniques in cephalopods is outside the scope of this document but the following four specific aspects are highlighted, along with a detailed discussion of anaesthesia in section 8.8 below. 
8.6.1 The operating room and environment. Surgical procedures should be performed in a dedicated room located close to the animals' home tanks to minimise animal transport, and induction of anaesthesia should take place in the surgical procedure room. Good surgical lighting is essential, but care should be taken that this does not cause localised heating of the animal.

Although a sterile operating environment is highly desirable to prevent cross-contamination from human to cephalopod (and potentially vice versa, see section 9), and to ensure a consistent scientific baseline, maintenance of sterility is not practical unless: sterile seawater and anaesthetic solutions are used throughout the operation, and the surgeon and any assistants are fully trained in sterile technique and are wearing appropriate clothing. General information on asceptic surgical techniques can be found in a LASA 2010 report. ${ }^{415}$

As a minimum, it is recommended that:

$i$. all apparatus or surgical instruments contacting the animal during surgery should be sterilised or at a minimum cleaned thoroughly with anti-bacterial and viricidal agents (e.g. diluted Halamid ${ }^{\S \S}$ or Virkon $^{* * *}$, F. Mark, pers. comm.);

ii. all material contacting the wound site such as surgical swabs and sutures should be sterile; and

iii. all personnel contacting the animal in the surgical environment should have cleaned their hands in accordance with aseptic technique and/or use sterile surgical gloves (blue nitrile gloves are recommended for handling animals, to avoid pale colours but these are not sterile and so should not be used for surgery);

$i v$. the surgical site should be cleaned; but at present there is no information on the effect of commonly used skin cleaning preparations on cephalopod skin integrity and healing;

$v$. the surgical site should be isolated as far as possible from any source of contamination (e.g. seawater/ anaesthetic solution).

8.6.2 Maintaining physiological function. Water used to irrigate cephalopod gills during surgery should be circulated, treated and monitored to maintain appropriate anaesthetic levels, oxygen, $\mathrm{pH}$, temperature and salinity, and to remove particulates. Pörtner et al. note that the sensitivity to hypoxia is greater in squid than either cuttlefish or octopus. ${ }^{417}$

Consideration should be given to using a cooled operating table (temperature monitored) in

\footnotetext{
${ }^{\S} \mathrm{N}$-chloro tosylamide, sodium salt; see: http://www.halamid.com/ Aquaculture-desinfection.htm, last visited August 2014.

"Peroxygenic acid; see Hernndez et al. ${ }^{416}$ and http://www2.dupont.com/Virkon_S/en_GB/, last visited August 2014.
}

combination with mantle perfusion with a chilled anaesthetic solution, as cooling can act as an adjuvant to anaesthesia, ${ }^{243,418}$ but should not be used as an anaesthetic alone. Cooling will also reduce metabolic rate, which may be advantageous when cardiorespiratory function is impaired as a result of anaesthesia.

The skin must be kept moist and this can be done with a sterile surgical drape moistened with sterile seawater. Care should be taken to minimise the potential for seawater to enter the wound site. Although anaesthesia to a depth sufficient to markedly suppress or stop ventilation may not be desirable from a physiological perspective, surgery involving incision of the mantle muscle and suturing may be very difficult in the absence of such marked suppression.

Whilst the efficacy of mantle perfusion with gassed anaesthetic/seawater solution for maintenance of blood gases may be questioned when there is severe suppression of cardiac function by the anaesthetic/anaesthesia, such perfusion may still be advisable to reduce anaesthetic wash out from the blood/tissues, to prevent desiccation of visceral structures and prevent/reduce hypoxic damage to critical tissues such as branchial hearts and gills. This may not be necessary for short duration $(<10 \mathrm{~min})$ minor surgical procedures, provided that full surgical anaesthesia can be assured.

8.6.3 Wound closure and healing. There are no studies assessing optimal materials to use for repair/closure of skin, muscle, connective tissue (e.g. hepatopancreas capsule), blood vessels or cranial cartilage in cephalopods, and publications do not often detail the materials used. The inclusion of such information in future publications is essential.

Closure techniques should follow normal practice for surgery in vertebrates, using round-bodied needles to reduce tissue trauma. Braided non-absorbable silk ${ }^{123,243}$, polyglyconate sutures ${ }^{282}$ and cyanoacrylate adhesives ${ }^{132,401,419-422}$ have been utilised. Several authors report that cephalopods will try to remove sutures with their arms (F. Mark, unpublished data) ${ }^{240}$ although this is not a universal finding and may reflect differences in surgical technique. Sutures sealed with cyanoacrylate have been used, but in some instances they may cause skin irritation (possibly due to being inflexible) and may not maintain integrity for longer periods of time in seawater.

An evaluation of five commonly used suture materials including braided silk, monofilament nylon and poliglecaprone has been undertaken in the mollusc Aplysia californica. ${ }^{423}$ All materials induced similar adverse skin and subcutaneous tissue reactions, but the authors recommended the use of braided silk because it induced a less intense granuloma reaction. This result was unexpected as in studies of aquatic vertebrates monofilament sutures are less reactive than braided silk. ${ }^{423}$ 
We would encourage anyone undertaking surgery in cephalopods to undertake histology on the wound site at the end of study, and include findings in any publications, with the aim of generating a database of good surgical practice. In addition to suture material, the most appropriate suture patterns (e.g. discontinuous, continuous) and needle size for skin closure in cephalopods has yet to be established, and this is knowledge especially important as the skin is particularly delicate in cephalopods.

Studies involving chronic implantation of devices such as archival ('data logging') tags ${ }^{185,417,424-426}$ need to consider possible chronic reaction of the tissues to the device and the impact that this may have on the normal behaviour of the animal. Mounts implanted in cuttlefish to support archival depth and temperature tags were well tolerated over a period of up to 5 months although post-mortem tissue thickening (possibly fibrosis) was observed around the implant and this increased in thickness with the duration of implantation. ${ }^{130,132}$ Chronic tissue reactions are also a potential issue for implanted identification tags (see section 5.7).

Descriptions of the natural course of wound healing following skin damage to the dorsal mantle in E. cirrhosa can be found in Polglase et al. ${ }^{189}$ Authors also found that bacterial infection of a mantle wound impairs healing. ${ }^{210}$ Healing and regeneration of the fin dermis in S. officinalis is described by Yacob et al. ${ }^{427}$ and regeneration of the mantle connective in several species of octopus by Sereni and Young. ${ }^{428}$

The arms in cephalopods have a remarkable capacity to heal and regenerate when a segment is removed by transection with the histological changes described in detail in O. vulgaris, E. cirrhosa, S. officinalis and $S$. pharaonis ${ }^{190,429-431}$ In $O$. vulgaris following transection of the distal $10 \%$ of the length of the arm as part of study to investigate regeneration, the exposed area was almost completely covered by skin in about 24 hours in some animals (T. Shaw and P. Andrews, unpublished data).

Cephalopods frequently injure arms, tentacles and fins in the wild; $;^{311,312,432-435}$ therefore a well-developed healing and regeneration mechanism is perhaps expected. This suggests that, provided sufficient time is allowed, surgical wounds, appropriately repaired, should heal successfully provided they do not become infected.

In addition to general anaesthesia surgery also requires the use of analgesics and this is discussed in section 8.9 below.

\subsection{Tissue biopsy}

Tissue biopsies for DNA isolation and PCR analysis for genotyping cephalopods have been obtained by taking small samples from the tip of an arm or tentacle with a sharp, sterile blade. ${ }^{436,437}$
The harms vs benefits of performing this procedure under general anaesthesia have not been assessed. We propose that irrespective of the use of general anaesthesia the arm tip should be treated with a local anaesthetic (in the absence of systemic analgesics). Only the absolute minimum amount of tissue should be taken (taking into account animal size) especially in cuttlefish as removal of large amounts of arm tissue transiently interferes with food manipulation and results in abnormal body posture. ${ }^{190}$ In squid and cuttlefish, the fins may provide an alternative site for biopsy as they also appear to heal rapidly and regrow. ${ }^{427}$ Although arms, fins and other tissues will regenerate, attention should be provided to avoid unnecessary sampling.

\subsection{General anaesthesia}

The Directive requires that 'procedures are carried out under general or local anaesthesia' unless anaesthesia is judged to be 'more traumatic to the animal than the procedure itself' and/or 'is incompatible with the purpose of the procedure' (Article 14\$2). 'Analgesia or another appropriate method' must also be 'used to ensure that pain, suffering and distress are kept to a minimum' (Article 14\$1), for example, peri- and postoperatively (see section 8.9 for further discussion). Furthermore, drugs that stop or restrict the ability of an animal to show pain (e.g. neuromuscular blocking agents) must not be used without an adequate level of anaesthesia or analgesia. When such agents are used, 'a scientific justification must be provided, including details on the anaesthetic/analgesic regime' (Article $14 \S 3)$. After completion of the procedure requiring anaesthesia 'appropriate action must be taken to minimise the suffering of the animal', for example, by use of analgesia and special nursing care (Article 14\$5).

During and after general anaesthesia and surgery, animals should be carefully and regularly monitored using a welfare monitoring scheme, and a record kept of observations and interventions to reduce or alleviate adverse effects. Of particular relevance is wound-directed behaviour but other potential welfare indicators are listed in Table 5. It is currently believed that operated animals should be housed individually at least until full recovery from general anaesthesia is assured, but this will clearly need particular consideration in gregarious species.

Many scientific procedures in cephalopods will require the use of general anaesthesia but despite more than 60 years of literature detailing studies involving anaesthesia, covering at least 17 agents, information is lacking on which agents in which species provide the most effective, humane general anaesthesia (i.e. which definitely blocks nociception and pain perception, 
generates no aversion, enables rapid induction and allows animals to recover quickly without adverse effects).

Recent studies have reviewed the use of agents claimed to have anaesthetic properties in cephalopods and criteria for evaluation of anaesthesia. ${ }^{8,61,131,418,438-440}$ In a recent contribution, isofluorane has been utilised to induce 'deep anesthesia' in $O$. vulgaris. ${ }^{441}$ However, more detailed studies are required to assess the application of this agent to induce anaesthesia in cephalopods.

In some cases, a range of behavioural responses is reported to occur during exposure of the animals to anaesthetic agents (i.e. inking, jetting, escape reactions, increased ventilation); ${ }^{208,243,438}$ however, it should be noted that most studies report immersing the animal directly in the anaesthetic at the final anaesthetic concentration, and this may not be the best technique to minimise trauma (see below). It should be also noted that criteria for general anaesthesia are not consistent between studies and that behaviours observed during induction and to assess depth may differ between species. ${ }^{439}$

Moreover, studies of general anaesthesia in cephalopods have investigated single agents and the familiar concept of 'balanced anaesthesia' involving more than one agent, used in mammalian studies (for reference see ${ }^{409,442}$ ) has not been explored in cephalopods (but see exceptions in Packard ${ }^{200,443}$ ).

8.8.1 General anaesthetic agents. Detailed descriptions of the commonly used anaesthetic agents (magnesium chloride, ethyl alcohol and clove oil) and their advantages and disadvantages in the common laboratory species can be found in recent reviews, ${ }^{8,439,440}$ and key features are described in Table 10.

Although a large number of agents have been investigated for anaesthetic efficacy in cephalopods, some are now considered unacceptable on either welfare or safety grounds (e.g. urethane), ${ }^{444}$ and so are not considered here. Similarly, we are not considering those utilised in a single experiment, and pending more evidence they are not considered herein.

Magnesium chloride $\left(\mathrm{MgCl}_{2}\right)$ is the most extensively studied and used agent, probably because it appears to be the least aversive. However, it disturbs haemolymph $\mathrm{Mg}^{2+}$ levels, and so may not always be appropriate, for example, when blood samples are required to investigate normal magnesium ion levels.

Furthermore, there has been a recurrent concern that $\mathrm{MgCl}_{2}$ may be acting at least in part as a neuromuscular blocking agent; ${ }^{41,445}$ but see comments in Graindorge et al. ${ }^{398}$ However to date there is no direct experimental evidence to support this concern in cephalopods, as reviewed in Andrews et al. ${ }^{8}$ The supposition may have arisen from the original use of magnesium chloride to relax small invertebrates prior to fixation. ${ }^{446}$

Messenger et al. ${ }^{447}$ concluded that $\mathrm{MgCl}_{2}$ exerts an effect on the central nervous system, and subsequent studies (G. Ponte, M.G. Valentino and P.L.R. Andrews, unpub. obs.) showed that electrical stimulation of efferent nerves in 'anaesthetised' $O$. vulgaris (as per criteria below) with $\mathrm{MgCl}_{2}(3.5 \%)$ evoked chromatophore contraction (arm and mantle), arm extension and mantle contraction showing a lack of effect at peripheral sites of motor control.

However, more recently, Crook et al. have shown that local subcutaneous and intramuscular injection of isotonic $\mathrm{MgCl}_{2}$ suppressed the afferent nerve activity in nociceptors activated by crushing a fin. ${ }^{11}$ This demonstrates that should such high local concentrations occur in animals immersed in $\mathrm{MgCl}_{2}$, it may have analgesic/local anaesthetic effects. Further studies of its mechanism of action are urgently required.

Ethanol is widely used as an anaesthetic agent in cephalopods, but it is not clear whether it blocks pain perception and/or is aversive. Some variability is reported in the response of animals exposed to it (see Table 10A), but this could reflect impurities in the different sources of ethanol used ${ }^{439}$ and other factors such as temperature.

For example, ethanol is considered effective in $O$. vulgaris, but has been reported to produce inking and escape reactions, ${ }^{243}$ suggesting that it is aversive. However, these reactions are not noted if the temperature is below $12^{\circ} \mathrm{C}^{448}$ Moreover, ethanol has been reported to be ineffective in cold water octopus species, as cited in Lewbart and Mosley following a personal communication from I.G. Gleadall. ${ }^{418}$ However, this observation seems contradicted by other personal experience on Antarctic octopods (F. Mark, pers. comm.).

Clove oil has been the subject of limited study so it is difficult to make a judgment about its use especially as there appear to be marked species differences in the response (see Table 10D). Further studies with clove oil, and its active constituent eugenol, are required to fully assess its utility as an anaesthetic for cephalopods.

Both $\mathrm{MgCl}_{2}$ and ethanol have been reported to induce general anaesthesia as defined in section 8.8.2 below to a level sufficient to perform relatively short duration ( $\sim 30 \mathrm{~min}$; see Table 10A,B) surgical or invasive procedures (see also Tables 8 and 9). However, it must again be emphasised that the analgesic, aversive and amnesic effects of these agents have not been studied in any detail and the molecular mechanism of their general anaesthetic action in cephalopods has not been elucidated.

In addition to further research on the agents themselves, it is also apparent from the limited data in Table 10 that there is emerging evidence of 
¿.

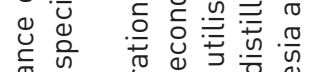

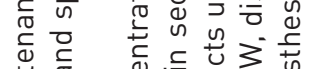

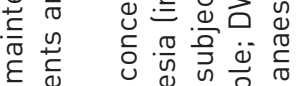

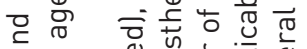

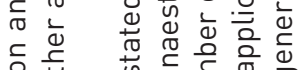

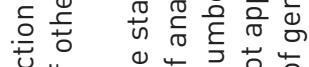

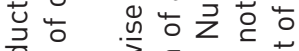

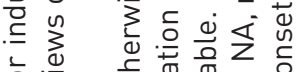

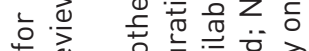

न

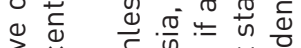

은

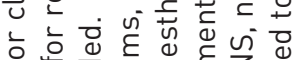

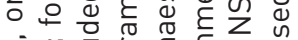

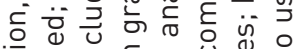

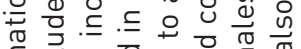

들

है.

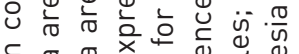

$\subseteq \frac{\pi}{U} \stackrel{x}{\Theta} .000$

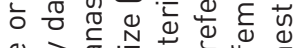

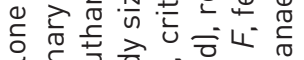

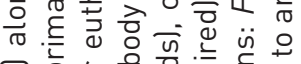

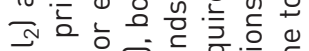

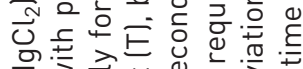

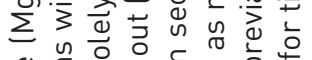

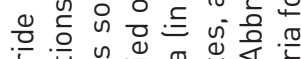

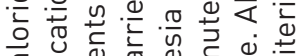

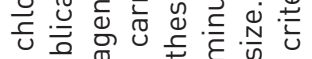

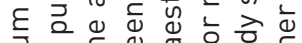

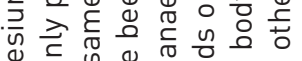

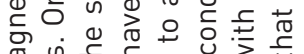

है

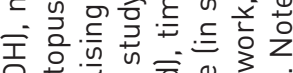

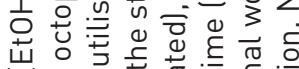

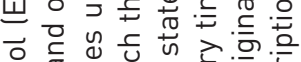

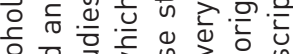

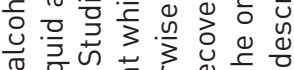

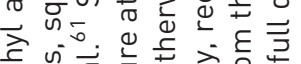

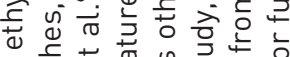

은 눙

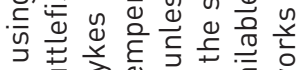

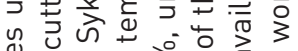

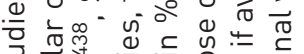

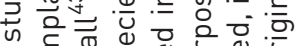

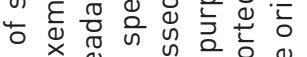

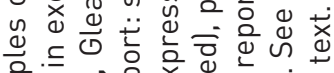

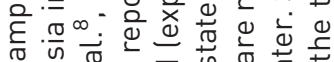

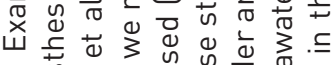

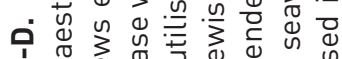

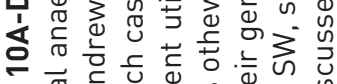

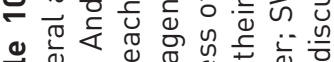

品 $\ddot{0}$

\begin{tabular}{|c|c|c|c|c|c|c|c|c|c|}
\hline 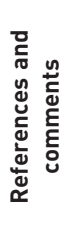 & 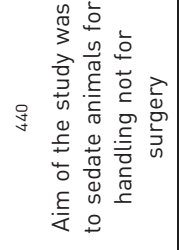 & 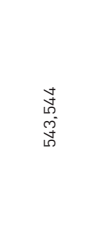 & 需 & $\stackrel{\infty}{\sim}$ & 号 & $\stackrel{\circ}{q}$ & \multicolumn{2}{|c|}{$\stackrel{\circ}{\circ}$} & \&े \\
\hline 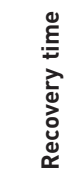 & 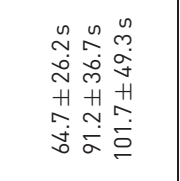 & $\tilde{z}$ & $\tilde{n}$ & 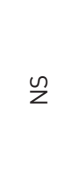 & $\stackrel{n}{z}$ & 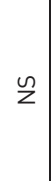 & \multicolumn{2}{|c|}{$\begin{array}{l}0 \\
0 \\
\vdots \\
\vdots \\
0\end{array}$} & $\begin{array}{l}\cdot \frac{c}{\bar{\varepsilon}} \\
\stackrel{\tilde{p}}{v}\end{array}$ \\
\hline 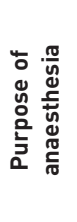 & 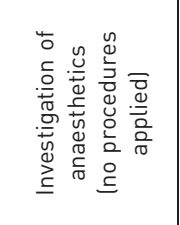 & 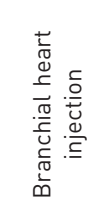 & 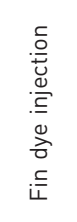 & 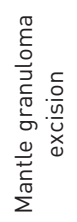 & 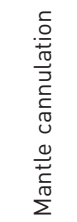 & 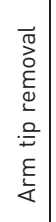 & \multicolumn{2}{|c|}{ 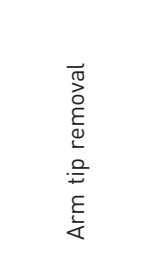 } & 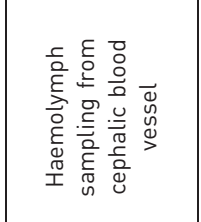 \\
\hline 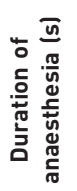 & $\stackrel{\infty}{\infty}$ & $\underline{n}$ & $\tilde{n}$ & $\begin{array}{l}\stackrel{c}{\varepsilon} \\
\frac{\checkmark}{f}\end{array}$ & n & 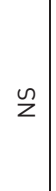 & \multicolumn{2}{|c|}{ n } & n \\
\hline 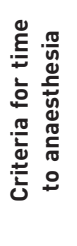 & 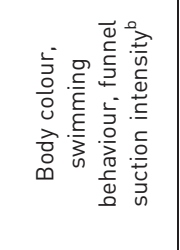 & 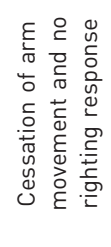 & $\tilde{n}$ & n & 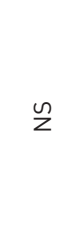 & 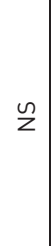 & \multicolumn{2}{|c|}{ 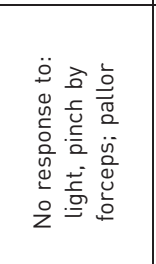 } & 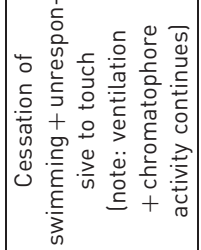 \\
\hline 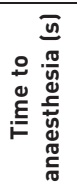 & 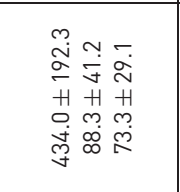 & n & $\stackrel{n}{z}$ & n & n & 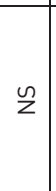 & \multicolumn{2}{|c|}{ n } & 웅 \\
\hline 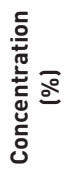 & $\stackrel{\circ}{-} \stackrel{\circ}{\sim} \stackrel{\circ}{m_{m}}$ & $\stackrel{\text { i }}{\mathrm{i}}$ & 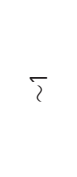 & 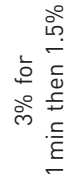 & $\stackrel{\llcorner}{\longrightarrow}$ & $\sim$ & \multicolumn{2}{|c|}{ 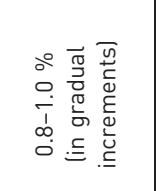 } & $\sim$ \\
\hline 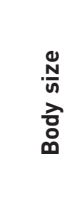 & 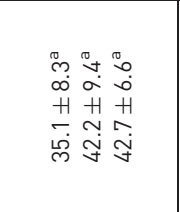 & 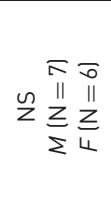 & 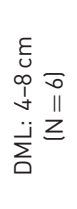 & స్సి & 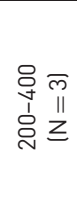 & 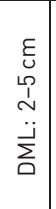 & 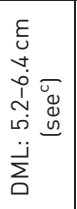 & 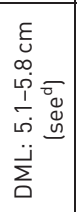 & $\frac{\vec{J}}{\frac{\vec{z}}{\sigma}}$ \\
\hline$\frac{\overline{0}}{\square}$ & 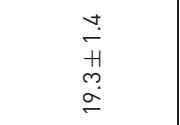 & $\tilde{n}$ & $\bar{N}$ & & $\begin{array}{l}\underset{\hat{N}}{\sim} \\
\text { }\end{array}$ & 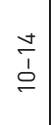 & \multicolumn{2}{|c|}{ 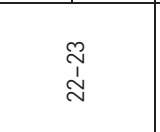 } & 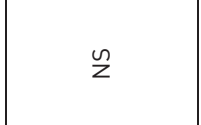 \\
\hline 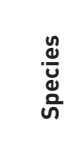 & 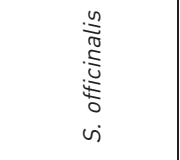 & 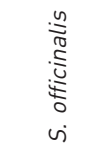 & 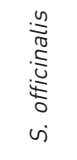 & 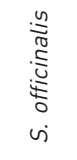 & 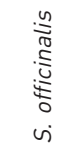 & 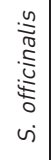 & 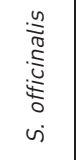 & $\begin{array}{l}\frac{n}{2} \\
\frac{0}{0} \\
\frac{0}{\pi} \\
\frac{\partial}{2} \\
v\end{array}$ & 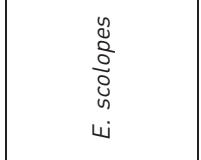 \\
\hline
\end{tabular}




\begin{tabular}{|c|c|c|c|c|c|c|c|c|c|}
\hline 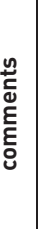 & 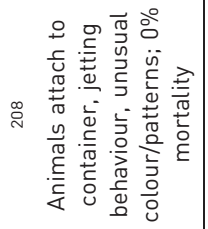 & 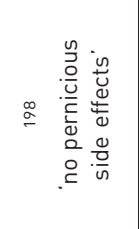 & 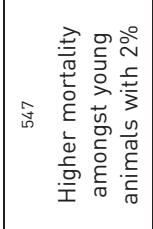 & $\stackrel{\bar{g}}{ }$ & $\stackrel{\stackrel{\circ}{g}}{2}$ & 号 & 总 & $\stackrel{\mathscr{్}}{~}$ & 号 \\
\hline 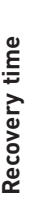 & n & 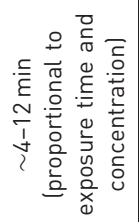 & $\begin{array}{l}\stackrel{n}{0} \\
\stackrel{\sim}{\sim} \\
\stackrel{n}{n}\end{array}$ & ñ & $\tilde{z}$ & n & n & n & $\begin{array}{l}\stackrel{n}{m} \\
\stackrel{+}{N}\end{array}$ \\
\hline & 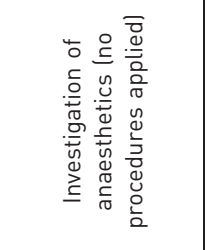 & 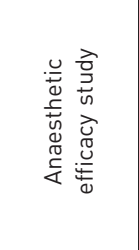 & 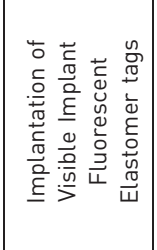 & 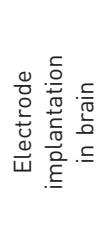 & 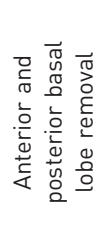 & 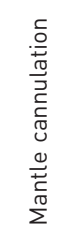 & 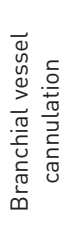 & 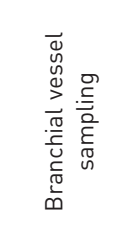 & 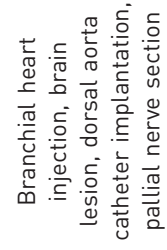 \\
\hline & $\frac{\frac{c}{\varepsilon}}{\frac{\vec{g}}{g}}$ & 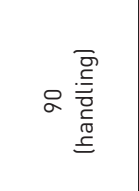 & 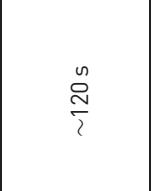 & n & n & n & n & n & 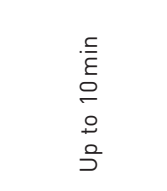 \\
\hline 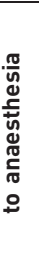 & 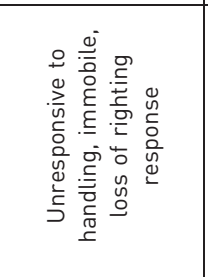 & 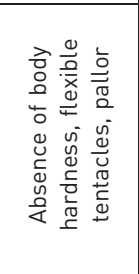 & 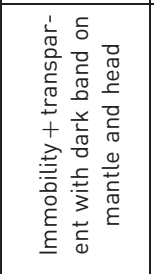 & n & $\tilde{z}$ & $\tilde{z}$ & n & 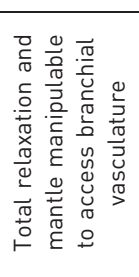 & 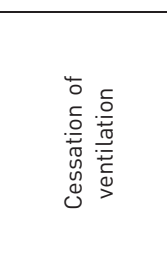 \\
\hline & 웛 & $\stackrel{\tilde{N}}{\mathrm{~N}}$ & $\stackrel{n}{n}$ & n & $\tilde{z}$ & $\underline{n}$ & n & n & $\begin{array}{l}\stackrel{\infty}{\oplus} \\
\stackrel{H}{+} \\
\stackrel{N}{N}\end{array}$ \\
\hline ळ & $-m$ & $\underline{p}$ & 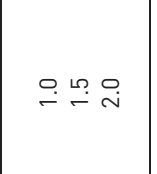 & $\begin{array}{l}\stackrel{\circ}{i} \\
\stackrel{i}{r}\end{array}$ & $\stackrel{\sim}{\sim}$ & $\sim$ & $\stackrel{\llcorner}{i}$ & 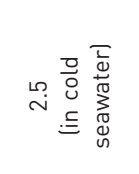 & $\stackrel{\circ}{i}$ \\
\hline 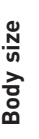 & 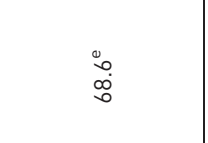 & 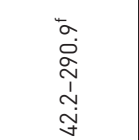 & 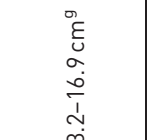 & 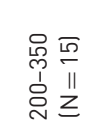 & 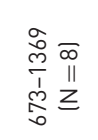 & 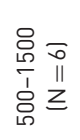 & 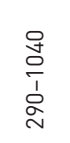 & 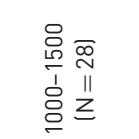 & 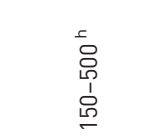 \\
\hline$\frac{\overline{0}}{\square}$ & \pm & n & n & n & 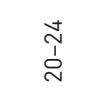 & $\begin{array}{l}\underset{\sim}{\Delta} \\
\grave{\sim}\end{array}$ & & $\stackrel{2}{\stackrel{2}{2}}$ & $\stackrel{\stackrel{\sim}{N}}{\underset{\sim}{\sim}}$ \\
\hline $\begin{array}{l}\frac{\mathscr{\Xi}}{\tilde{u}} \\
\text { nँ }\end{array}$ & 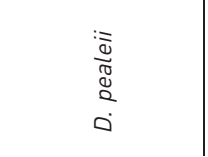 & 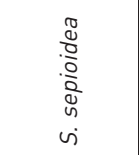 & 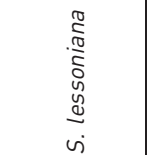 & $\begin{array}{l}\frac{n}{2} \\
\frac{n}{5} \\
3 \\
3 \\
0\end{array}$ & $\begin{array}{l}\frac{n}{2} \\
0 \\
3 \\
3 \\
0\end{array}$ & $\begin{array}{l}\frac{n}{2} \\
0 \\
3 \\
3 \\
0\end{array}$ & $\begin{array}{l}\frac{2}{2} \\
0 \\
0 \\
3 \\
0\end{array}$ & $\begin{array}{l}\frac{n}{2} \\
\frac{n}{0} \\
\frac{3}{3} \\
0 \\
0\end{array}$ & $\begin{array}{l}\frac{n}{5} \\
0 \\
3 \\
3 \\
0\end{array}$ \\
\hline
\end{tabular}




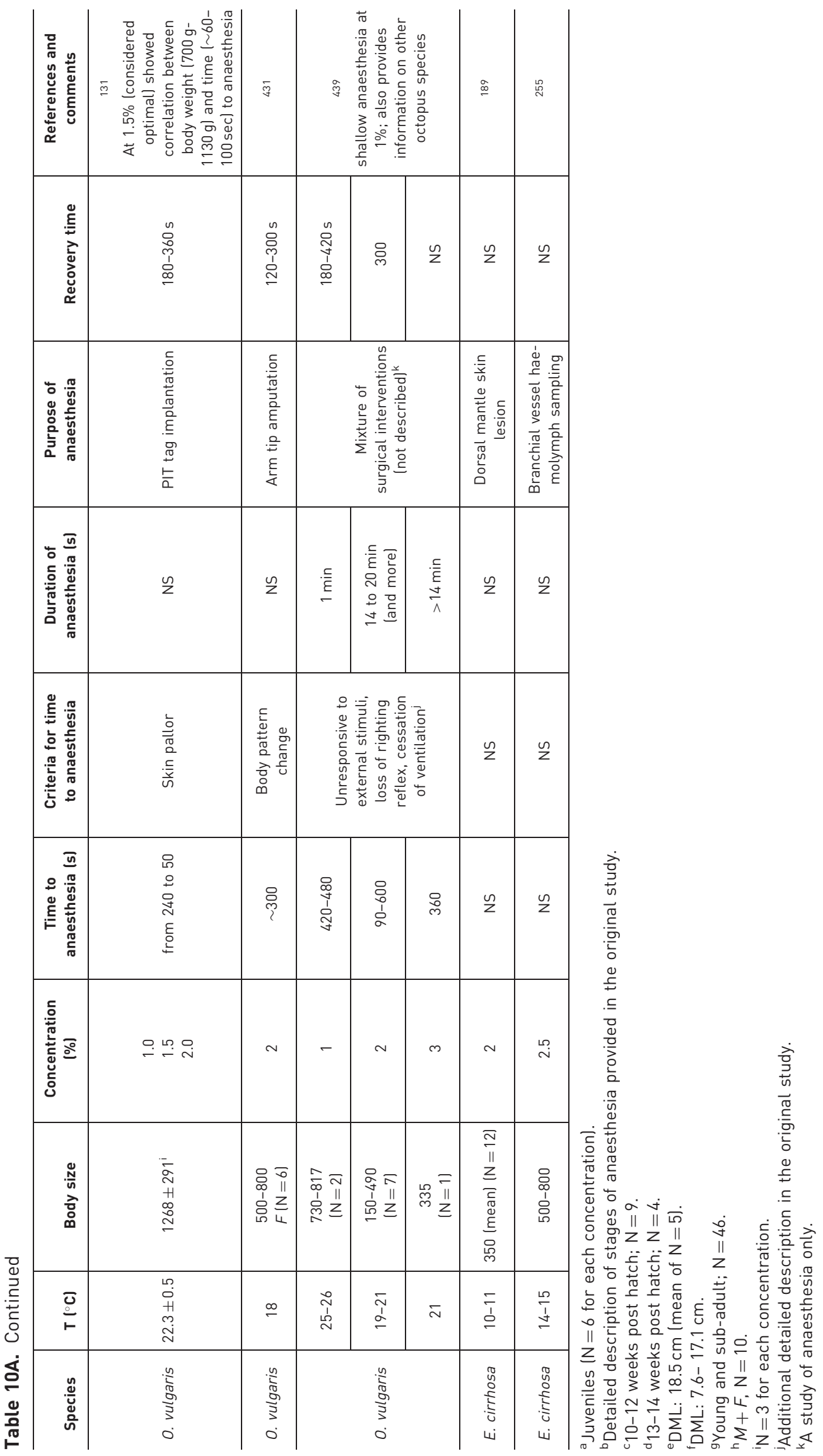




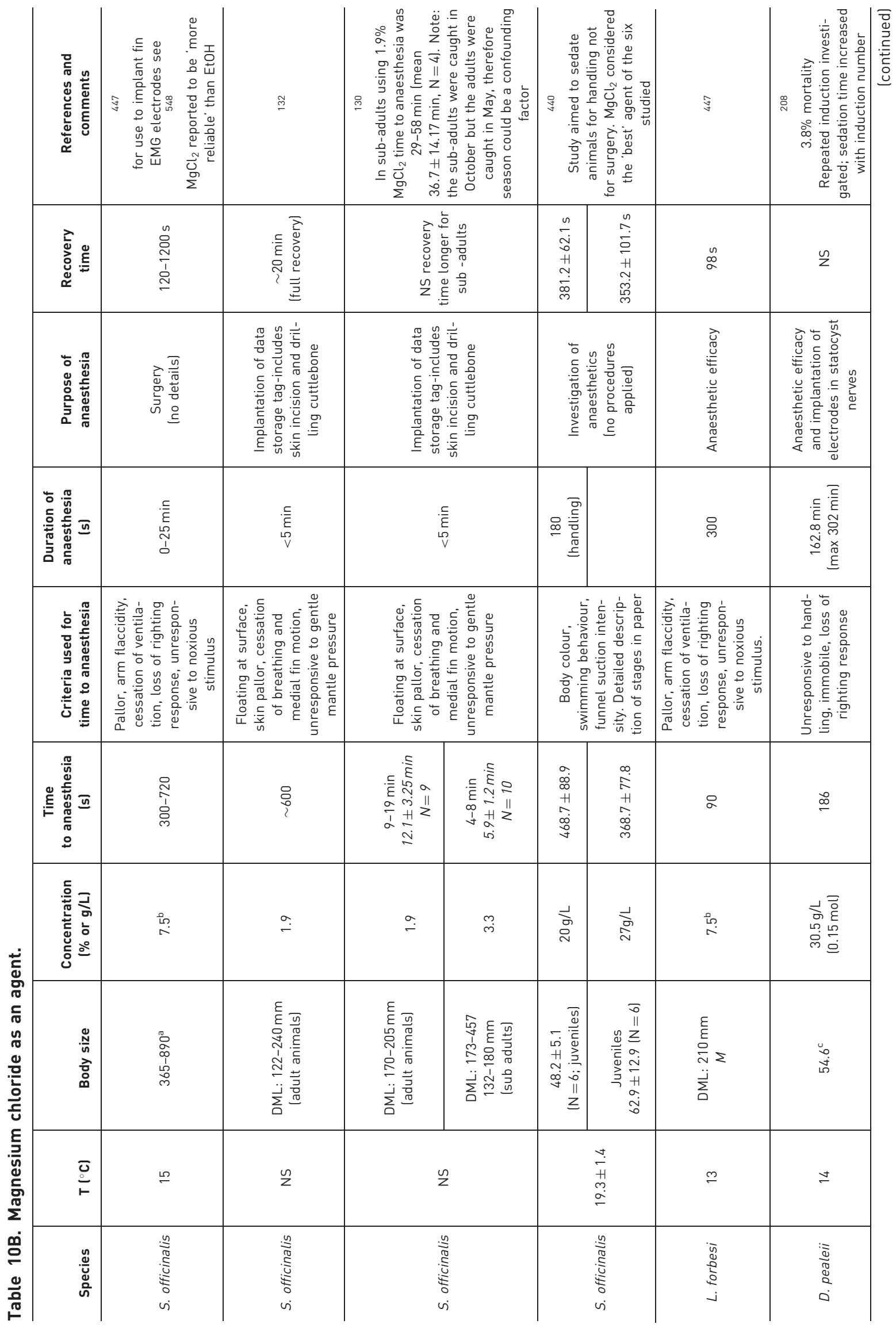




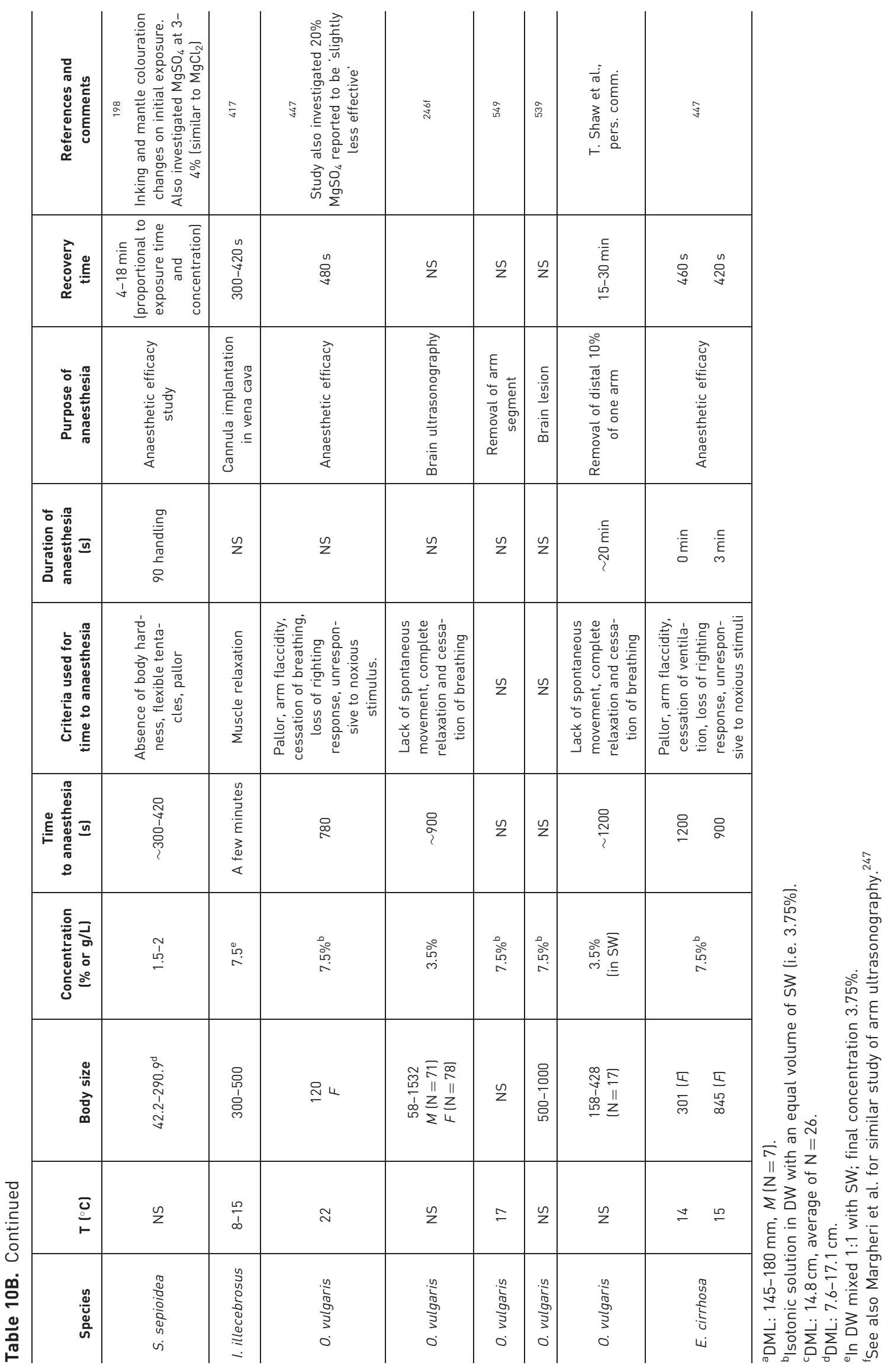




\begin{tabular}{|c|c|c|c|}
\hline 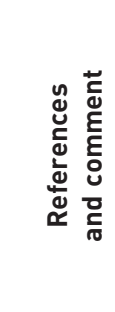 & 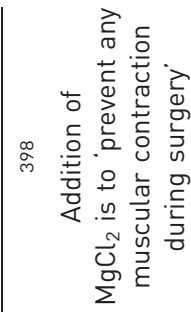 & $\stackrel{\text { న }}{\sim}$ & 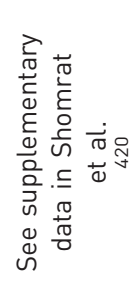 \\
\hline 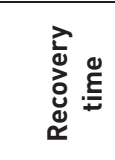 & $\Omega$ & $\tilde{z}$ & 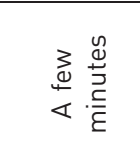 \\
\hline 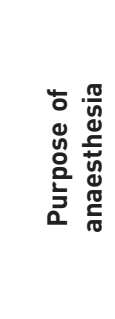 & 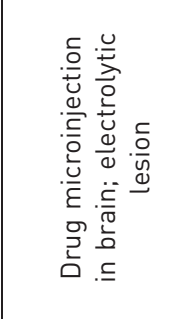 & 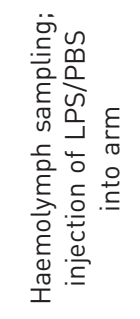 & 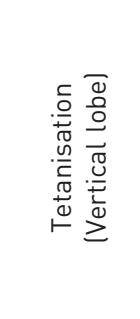 \\
\hline 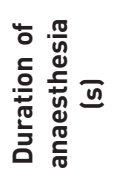 & $\stackrel{N}{z}$ & $\underline{n}$ & $\underline{n}$ \\
\hline 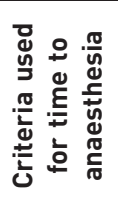 & $\stackrel{\Omega}{z}$ & $\tilde{Z}$ & $\stackrel{\Omega}{z}$ \\
\hline 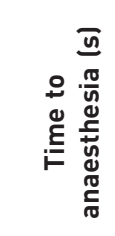 & $\begin{array}{l}\text { व } \\
\vdots \\
\vdots \\
\infty\end{array}$ & $\begin{array}{l}\text { ○ } \\
\text { ? }\end{array}$ & 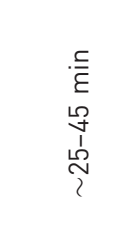 \\
\hline 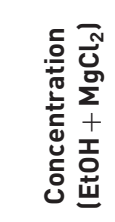 & $\begin{array}{l}\stackrel{8}{\circ} \\
\stackrel{2}{\infty} \\
\stackrel{+}{+} \\
\stackrel{0}{N}\end{array}$ & $\begin{array}{l}\sum_{\varepsilon} \\
\llcorner \\
\llcorner \\
+ \\
\stackrel{0}{-}\end{array}$ & $\begin{array}{l}\sum_{\varepsilon} \\
\llcorner \\
\llcorner \\
+ \\
+ \\
\stackrel{\circ}{\circ}\end{array}$ \\
\hline $\begin{array}{l}\frac{N}{n} \\
\frac{N}{0} \\
\text { वे } \\
0\end{array}$ & $\frac{\stackrel{O}{0}}{1}$ & 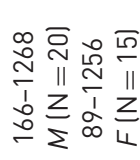 & 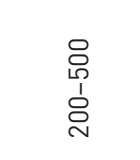 \\
\hline$\frac{\overline{0}}{\circ}$ & $\stackrel{\Omega}{z}$ & 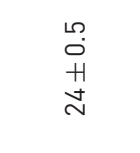 & $\Sigma$ \\
\hline $\begin{array}{l}\frac{\mathscr{E}}{\tilde{\Xi}} \\
\text { nू }\end{array}$ & 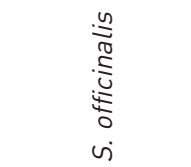 & $\begin{array}{l}\frac{n}{2} \\
0 \\
0 \\
3 \\
0 \\
0\end{array}$ & $\begin{array}{l}\frac{2}{2} \\
\frac{2}{0} \\
3 \\
3 \\
0\end{array}$ \\
\hline
\end{tabular}




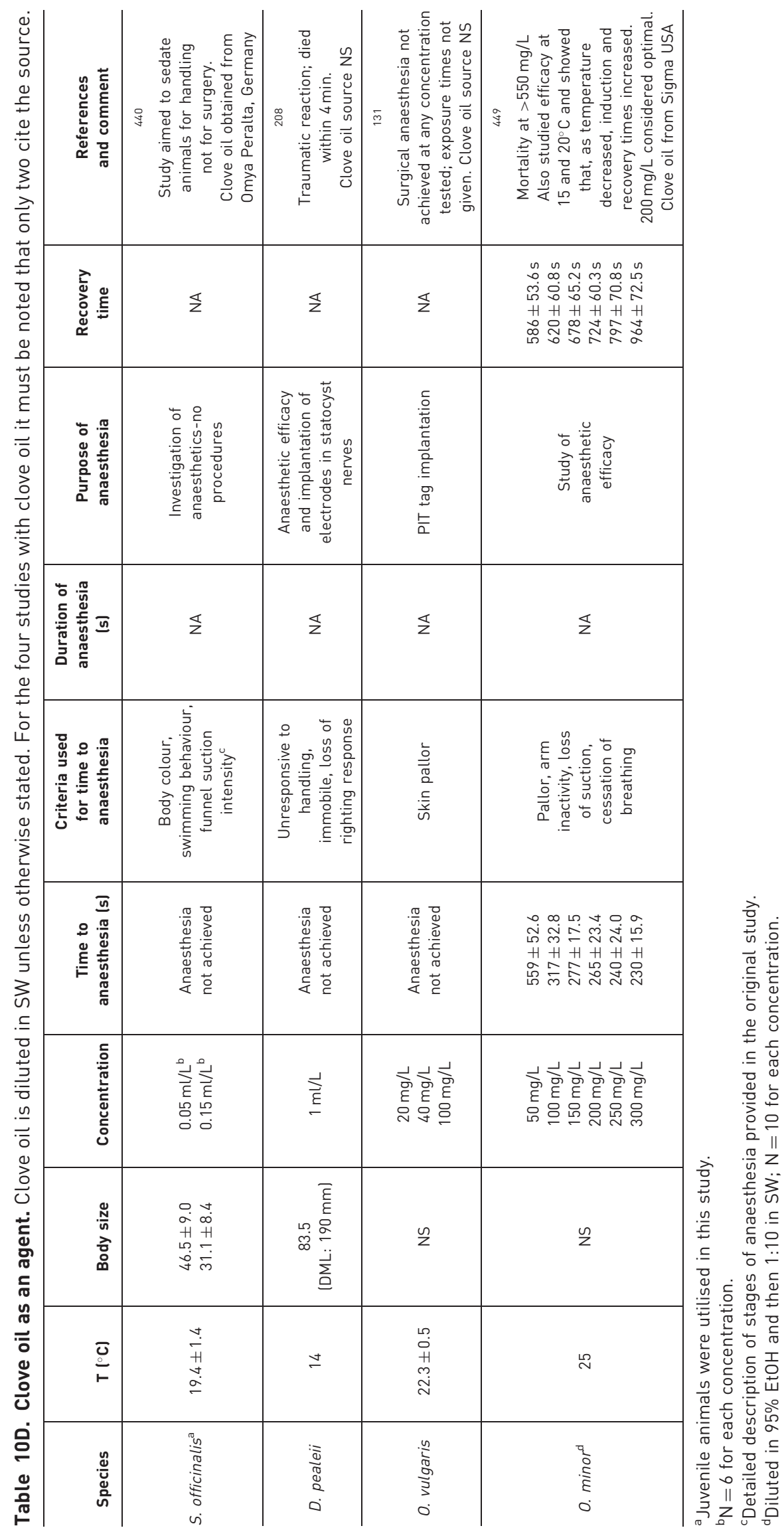


differences in 'efficacy' of these agents according to age $^{102}$ and body weight ${ }^{131}$ of animals; and temperature of the solution. ${ }^{449}$

It should also be noted that the majority of systematic studies of anaesthesia in cephalopods involve the commonly used laboratory species, and particular care should be taken when attempting to anaesthetise other species, as responses to the same agent may differ markedly. For example, benzocaine (ethyl p-amino benzoate) produces a violent reaction followed by death in the squid $D$. pealeii, ${ }^{208}$ but violent reactions are not reported in the octopus E. dofleini where it has been used for euthanasia. ${ }^{450}$

8.8.2 Criteria for general anaesthesia. A detailed discussion of criteria for general anaesthesia in cephalopods, based on a review of recent studies on cuttlefishes, squid and octopuses is now available. ${ }^{8,440}$ They will only be outlined here, and further work is needed to define species-specific criteria for general anaesthesia.

Quantification of the physical (externally visible) parameters listed below might enable assessment of putative 'stages' or 'planes' of anaesthesia, and studies in cuttlefish ${ }^{440}$ and octopuses ${ }^{131,243,449}$ illustrate this approach. In particular, ventilation depth and frequency and chromatophore activity are the parameters most amenable to continuous real time quantification to monitor the onset of 'anaesthesia'. Currently there are no studies of electroencephalogram (EEG) activity in cephalopods under general anaesthesia, and all the parameters illustrated below rely on the assumption that the agents used to induce anaesthesia do not act only as neuromuscular blocking agents.

(i) Decreased or absent response to a noxious stimulus. This is the most important test that the animal is sufficiently anaesthetised for surgery or other procedures to commence. Without blocking pain perception general anaesthesia cannot be considered to have been achieved. Studies have used a mechanical stimulus (e.g. a pinch) applied to the arm, mantle or supraorbital skin as a test of insensibility to a noxious stimulus. ${ }^{132,243,447}$ The selection of this type of challenge appears appropriate, as it is now known that cephalopods possess mechano-sensitive peripheral nociceptors, ${ }^{10,11}$ and in future this knowledge will enable the identification of betterdefined stimuli to test for insensibility. Assuming that there is evidence to suggest that the animal is insensible, the following criteria should also be evaluated.

(ii) Depression of ventilation. The initial reaction to exposure to the anaesthetic may be an increase in depth and frequency of mantle contractions with attempts to eject the solution via the siphon. Subsequently the frequency decreases progressively with time after exposure, and the coordination between the mantle and siphon become uncoordinated. With prolonged exposure, mantle and siphon contractions cease.

(iii) Decreased chromatophore tone. Although animals become pale overall with increasing time of exposure to the anaesthetic, flashing colour changes have been reported on initial exposure to anaesthetic agents (e.g. in D. pealeii ${ }^{208}$ or in $S$. officinalis ${ }^{440}$ ). The overall paling of the animal is indicative of a decrease in the central nervous system drive to the chromatophore motorneurones. ${ }^{201}$

(iv) Decreased locomotor activity, arm/tentacle tone and sucker adhesiveness. The initial reaction to anaesthetic exposure may be an increase in activity (i.e. agitation, for review see Gleadall ${ }^{439}$ ), but activity gradually decreases, including swimming activity and fin movement, as for example in cuttlefish and squid. ${ }^{132,451}$ Octopuses will tend to settle on the bottom of the tank as the arms and suckers begin to lose tone and adhesion, but anaesthetised cuttlefish may float near the surface of the tank. ${ }^{132}$ The arms should be flaccid and readily manipulated. ${ }^{449}$

(v) Loss of normal posture and righting response. As animals (e.g. squid and cuttlefish) become deeply 'anaesthetised' they lose the ability to maintain a normal position in the water column or adopt an abnormal position with the arms, head and mantle at angles not normally seen in conscious animals. For example, in squid and cuttlefish the arms and head may appear unsupported by the mantle collar muscles; octopuses adopt a flattened appearance on the floor of the tank rather than the usual posture with the head raised. In addition, animals placed on their dorsal surface make no attempt to right themselves. The righting response returns after ventilation and chromatophore activity return, ${ }^{243}$ and this is probably a good indicator of overall recovery from anaesthesia, as both effects require complex coordination of neuromuscular activity.

(vi) Absence of a response to light. The absence of a reaction to a strong light has been used as one sign of general anaesthesia (e.g. in Messenger et al. ${ }^{447}$ ), but this is poorly characterised.

8.8.3 Induction. A common practice for induction is to immerse the animal in the anaesthetic solution (made up in seawater) at its final concentration. However, to 
minimise trauma it is preferable to expose the animal to a rising concentration of the agent (as for example in Yacob et al. ${ }^{427}$ ), which will also allow any adverse reaction to be quickly identified.

The animal should remain completely immersed in the anaesthetic solution for rapid effect. In addition, the use of a specialised closed anaesthetic chamber should be considered. The chamber could also be used as a transport box from the home tank to the operating room, and it may be possible to habituate at least some species (e.g. octopus and cuttlefish) to the box and train them to enter. This will reduce stress to the animals, and a closed chamber will prevent octopus escaping. As a general rule, animals should always be transported in seawater and movement should be minimised.

Anaesthetic solutions should always be freshly made, using filtered seawater, which is gassed (preferably with oxygen rather than air) and equilibrated to home tank temperature before immersing the animal. It is not good practice to anaesthetise an animal in a solution that has been used to anaesthetise another animal, as the water may contain chemical alarm signals. Animals must not be left immersed in an anaesthetic solution in which they have inked.

Although limited in scope, the pre-anaesthetic sedation technique applied by Packard ${ }^{200}$ has not been followed by any other systematic study of methods to minimise the stress of general anaesthesia in cephalopods, as has been done for fishes. ${ }^{452}$

Moreover, there is no general agreement about whether cephalopods should be deprived of food prior to anaesthesia. Some studies remove food for 24 hours ${ }^{440}$ while others do not. ${ }^{208}$

We are aware of only one report of food regurgitation by $S$. sepiodea during 'anaesthesia' in magnesium sulphate, ${ }^{198}$ although defaecation is relatively common.

In view of the above, the anaesthetic protocol for cephalopods requires careful planning, including considering whether or not to withdraw food and, if so, for how long.

8.8.4 Maintenance and monitoring. Once the animal is fully anaesthetised (see above for criteria), it will usually be necessary to remove it from the anaesthetic chamber to perform a procedure. Anaesthesia must be maintained for the entire duration of the procedure, and physiological functions supported. ${ }^{282,453,454}$

Several authors describe apparatus (adapted from fish anaesthetic apparatus) for maintenance of anaesthesia during surgery (e.g. for cuttlefish: ${ }^{132,418,454}$ for squid: ${ }^{417,451}$ ), and these could also be better tested, and also adapted for octopus.

Marked suppression or cessation of ventilation (indicated by mantle/siphon contraction) is a common feature of general anaesthesia in cephalopods, so it is essential that the mantle is perfused with oxygenated seawater/anaesthetic. Such perfusion will only be effective if the branchial and systemic hearts continue to function, but little is known of cardiac function under anaesthesia. However, it has been observed that heart rate is very low in O. vulgaris anaesthetised with $\mathrm{MgCl}_{2}$ (M.G. Valentino and P.L.R. Andrews, unpublished observations) and in animals immersed in cold water. ${ }^{243}$

Monitoring of physiological function under anaesthesia and during surgery is clearly an area requiring research so that the extent of hypoxia/hypercapnia is known and its impact on post-operative recovery and procedures can be assessed. However, O. vulgaris appears to be able to recover rapidly from protracted periods of apnea ${ }^{150}$ and Pörtner et al. ${ }^{417}$ comment that cuttlefish and octopus are less sensitive to hypoxia than squid (as might be expected from their different lifestyles). Doppler ultrasound (e.g. as in D. Fuchs and G. Ponte, unpublished observations; Vevo 2100 Visualsonics, The Netherlands) offers the best technique for monitoring cardiovascular function, but non-invasive methods for real time monitoring of blood gases (such as oximetry) and metabolic status (e.g. NMR spectroscopy as in Melzner et al. ${ }^{455}$ ) need further development.

8.8.5 Recovery. The mantle should be flushed of residual anaesthetic solution and the animal then placed in clean aerated/oxygenated seawater. Ventilation can often start without intervention (depending upon the duration of anaesthesia), but gentle massage of the mantle is frequently used in cuttlefish, squid and octopods until spontaneous ventilation, as indicated by mantle and siphon movements, restarts. Other functions (sucker adhesion, chromatophore tone, righting) recover after ventilation recommences, usually in the reverse order to which they were lost.

Many studies monitor the time at which various functions return, but the time taken for full recovery of normal function from particular anaesthetic protocols is not known and, as most studies of anaesthesia do not involve surgery, the impact of surgery upon recovery is not known, but see Shomrat et al ${ }^{420}$ As currently assessed, recovery appears rapid (less than 15 mins, e.g. in $^{243,420,440}$ ) and dependent on the procedure(s) performed, animals will usually take food quickly after 'anaesthesia' when returned to their home tank. ${ }^{395,420}$ However, further monitoring criteria are needed to ensure that animals have fully recovered from the anaesthesia and any surgical procedure. If recovery from anaesthesia is as rapid as appears to be, and the anaesthetic agents used do not have residual analgesic properties, it is vital that suitable analgesics are administered - which at this time of writing may best be done 
by infiltrating surgical sites with local anaesthetic (see next section below).

\subsection{Analgesia and local anaesthesia}

Directive 2010/63/EU, Article 14\$4, requires 'an animal which may suffer pain once general anaesthesia has worn off, shall be treated with pre-emptive and post-operative analgesics or other appropriate pain-relieving methods, provided that it is compatible with the procedure'.

At the time of writing, there is no information on the efficacy of any analgesics in cephalopods, although both ketoprofen and butorphanol have been recommended. ${ }^{445}$

As nociceptors have been recently studied in cephalopods, ${ }^{10,11}$ it should be possible to investigate the efficacy of systemically administered substances for potential analgesic activity. In addition to identification of mechano-nociceptors, Crook et al. also showed that injury to a fin in squid induced spontaneous activity and sensitisation at sites distant from the lesion including the contralateral body; ${ }^{11}$ similar sensitisation of both the wound site and at distant sites has been reported in octopus. ${ }^{10}$ This implies that potentially surgery at any surface site (but possibly anywhere including the viscera) could evoke a more general sensitisation of nociceptors. If this is the case, it is essential that suitable analgesic agents are quickly identified.

In the absence of the availability of systemic analgesics, it is recommended that local anaesthetics are used to produce localised analgesia either by infiltration into a wound site or local nerve block. Xylocaine $(2 \%)$ and mepivacaine $(3 \%)$ have both been shown to be effective in producing a block of transmission in the arm nerve cord lasting at least 1 hour (G. Ponte, M.G. Valentino and P.L.R. Andrews, unpub. obs.). ${ }^{149,456} \mathrm{It}$ should be noted that local anaesthetics acting on the fast tetrodotoxin (TTX) sensitive voltage gated sodium channels may not be effective in species such as the blueringed octopus which possesses endogenous TTX. ${ }^{457}$

Selective nerve block with infiltration of a local anaesthetic should also provide an interim means of preventing more generalised nociceptor sensitisation (see above), as it has been demonstrated that afferent nerve block by injected isotonic $\mathrm{MgCl}_{2}$ prevented both local and distant sensitisation. ${ }^{11}$

\subsection{Fate of animals at the end of a procedure}

At the end of a procedure as defined in section 8.1 above, a decision about the fate of the animal is required. The Directive identifies three possibilities:

Humane killing: Article 17 requires that 'at the end of a procedure' an animal must 'be killed when it is likely to remain in moderate or severe pain, suffering, distress or lasting harm'. Other animals should also be humanely killed at the end of procedures, unless 'a decision to keep an animal alive' has been taken 'by a veterinarian or another competent person', when the possibilities described below should be considered. Methods of humanely killing cephalopods are discussed in section 8.11 below.

Release or re-homing: An animal 'may be... returned to a suitable habitat or husbandry system appropriate to the species', provided that: 'its state of health allows it; there is no danger to public health, animal health or the natural environment; and appropriate measures have been taken to safeguard [its] well-being' (Article 19). This could include release to the animals' natural environment, transfer to public aquaria, educational or other competent holding facilities. However, in general, cephalopods should not be returned to the wild, except in studies where, following a procedures, animals are released immediately at the exact location where they were captured, during a relevant season for migratory species, having been certified fit by a veterinarian or other competent person (Articles $17 \S 2$ and 19). Any requirements of other national and international legislation regarding the release of animals to the wild must also be met.

Re-use: Animals may also be considered for re-use, provided certain conditions are met.

Article 16 defines re-use as 'use of an animal already used in one or more procedures, when a different animal on which no procedure has previously been carried out could also be used. By definition, re-use applies to situations in which the objectives of the first and second procedures are unrelated. ${ }^{\dagger \dagger}$

Reuse is only permitted when:

- 'the actual severity of the previous procedure(s) was 'mild' or 'moderate';

- the animal's 'health and wellbeing has been fully restored;

- the further procedure is classified as 'mild', 'moderate' or 'non-recovery' (see section 8.1); and

- the proposed re-use 'is in accordance with veterinary advice, taking into account the lifetime experience of the animal'.

\footnotetext{
${ }^{1}$ This distinguishes reuse from situations where the scientific objective can only be achieved by using the same animal in more than one procedure (this is known as continuing use, although the term is not used in the Directive).

THFor further discussion and examples, see pp. 8-10 in EU National Competent Authorities endorsed document (2011), available at: http://ec.europa.eu/environment/chemicals/lab_animals/ interpretation_en.htm
} 


\subsection{Methods of humane killing}

The ultimate fate of cephalopods in the majority of studies will be humane killing. All personnel involved in humane killing should be trained and be familiar with the principles of good practice, such as those set out by Demers et al. ${ }^{458}$

Article 6 requires that, whenever animals are killed:

- it should done with minimum pain, suffering and distress by a competent person; and

- one of the methods of killing listed in Annex IV (section 3) of the Directive should be used, followed by confirmation of death, using one of the methods listed in Annex IV section 2.

However, whilst methods listed for fish might be applied to cephalopods, Annex IV offers no specific guidance on methods for humanely killing cephalopods. Although the $\mathrm{CCAC}^{459}$ take the view that the priority is a rapid loss of consciousness, these guidelines concur with the view of Hawkins et al. from a discussion of $\mathrm{CO}_{2}$ killing in vertebrates that it is more important to avoid or minimise pain and distress than it is to ensure rapid loss of consciousness' (p. 2). ${ }^{460}$

We have described some possible methods below, but the efficacy, and level and nature of any suffering caused, have not been comprehensively evaluated for all of these techniques, and further research is needed. Animals should not be killed in the rooms used to house other animals nor within sight of conspecifics. It must also be ensured that blood or chemical alarm signals cannot be detected by other animals (e.g. by entering the water system). Whichever method is used in a given species, the potential impacts of factors such as body weight, age, sex, season and water temperature on the efficacy of the method must be considered.

8.11.1 Chemical methods. Chemical methods for killing cephalopods are based on an overdose of anaesthetic agents, by using either a higher concentration and/ or longer exposure time than that needed for anaesthesia. As also outlined above, these methods all have the potential to cause adverse effects prior to unconsciousness, such as skin or eye irritation, or sensation of asphyxia. To reduce and avoid any such suffering, animals should be exposed to a gradually rising concentration of the anaesthetic, and not directly immersed in a solution at the full concentration needed to cause death. In tropical and temperate species, cooling may be used as an adjuvant to the anaesthetic for humane reasons, and could also reduce post-mortem tissue damage if tissue is required for in vitro studies.

A previous review of this topic proposed the following protocol as suitable for S. officinalis, D. pealei, O. vulgaris and E. cirrhosa: 'At least 15 minutes immersion in $\mathrm{MgCl}_{2}$, with a rising concentration [optimal rates to be determined], ending with a final concentration of at least $3.5 \%$ in the chamber used for humane killing; possibly enhanced by using chilled solutions or with the clove oil's active ingredient eugenol, followed by immediate mechanical destruction of the brain' (Andrews et al., p. 61). ${ }^{8}$

It was further proposed that if the brain was needed, the immersion period should be extended to more than 30 minutes to ensure unconsciousness as required in Directive 2010/63/EU (Annex IV, 1(a)) prior to removal of the brain. If the brain is not removed for study, confirmation of permanent cessation of circulation (see 8.12 below) is also considered as a possible method for completing killing according to Directive 2010/63/EU (Annex IV, 2(a)).

8.11.2 Mechanical methods. When carried out by highly skilled operators, death by mechanical destruction of the brain takes only a few seconds, but the nature and degree of any suffering is unknown. For this reason we take the view that cephalopods should not be killed by this, or any other, mechanical method without prior sedation/anaesthesia. However, it may be possible to utilise a 'mechanical method' if it can be justified and is authorised by the National Competent Authority as a specific regulated procedure within a project application.

Electrical methods such as 'Crustastun' used for humanely killing crustacea, such as lobsters and crabs, ${ }^{461}$ might also be considered and evaluated for their suitability in terms of animal welfare. It will be particularly challenging to develop humane methods for use when the brain is required intact, but where the use of anaesthesia may be a confounding factor.

\subsection{Confirmation of death}

Use of a method for confirming death following humane killing is mandatory, and options are listed in Annex IV $\$ 2$ of the Directive. Two of the methods listed, i.e. 'dislocation of the neck' and 'confirmation of onset of rigor mortis' are impossible for cephalopods - the latter because it does not occur in cephalopods.

This leaves three possible methods: $i$. confirmation of permanent cessation of the circulation; $i$. destruction of the brain; or iii. exsanguination.

Confirmation of permanent cessation of the circulation and exsanguination. Octopuses, cuttlefish and squid have two branchial hearts that move blood through the capillaries of the gills. ${ }^{462}$ A single systemic heart (the only one in nautiloids) then pumps the oxygenated blood through the rest of the body. The heart(s) may continue beating for some time after permanent cessation of breathing, so transection of the dorsal aorta/vena cava may be used. Transection of the dorsal aorta/vena cava will be effective in inducing exsanguination if the systemic heart is able to 
pump effectively (i.e. the anaesthetic used does not supress cardiac function); also note that the systemic heart is distension sensitive. ${ }^{463}$ Finally, the possibility of transection of the branchial aorta afferent to the heart, at the level of the auricle, should be further explored considering the easy access to them through the mantle cavity nearby the gills (G. Ponte and G. Fiorito, pers. comm.).

The effectiveness of exsanguination as a method of killing is not known.

Freezing (below $-18^{\circ} \mathrm{C}$ for several hours) after killing may be a further means of confirming cessation of circulation and hence death that does not necessarily entail destruction of the body.

Destruction of the brain may be difficult to ensure in some species because of the location and relatively small size (e.g. Nautilus sp.) although this can be overcome by training and a detailed knowledge of the cranial anatomy of the relevant species.

The methods used for humane killing and confirmation of death should always be included in publications.

Article 18 of the Directive requires member states 'to facilitate the sharing of organs and tissues of animals killed' where appropriate. Researchers should be encouraged to use tissue from animals killed in other projects for in vitro research (e.g. tissue bath pharmacology), rather than killing an animal specifically/only to obtain tissues; and consideration should be given to setting up banks of frozen and fixed tissue to optimise animal use.

\section{Risk assessment for operators}

This section will focus on the potential risks from the direct handling of cephalopods in a laboratory setting, but will not cover the more generic risks associated with working in either a laboratory (e.g. tissue fixatives, reagents) or a marine aquarium environment (e.g. tank cleaning agents, slipping, electricity in a wet environment). However, all personnel involved in research should be appropriately educated, trained and competent to perform any task relevant to the research. Personnel should be actively involved in risk assessment and management, and incident reporting encouraged.

\subsection{Personnel to consider}

The following personnel should be considered and their risks assessed:

a. Fishermen, divers or others responsible for the capture of cephalopods in the wild. Although such people may not be employees of the institution/facility, as far as possible, the institution/facility should be assured that safe practices are being employed. The Directive specifies that, in cases where justification is provided, to obtain animals from the wild 'competent persons' (Article 9) should be involved. As part of competency assessment issues related to health and safety practices of cephalopod capture should be explored.

b. Animal carers and technicians. This includes anyone involved in cleaning and feeding animals; cleaning animal rooms and equipment (e.g. tanks, filters).

c. Animal technologists or research laboratory technicians. These are personnel who may be involved in manipulating animals during experiments, sampling biological fluids, euthanasia and necropsies.

d. Principal and other Investigators: All personnel involved in performing research experiments including in vivo regulated procedures and in vitro handling of live tissue.

e. Designated veterinarian (or other suitably qualified expert).

f. Personnel responsible for the disposal of animal remains.

\subsection{Risk identification, prevention and protection}

9.2.1 Physical risk. Bites of cephalopods are produced by the hard beak-like jaws associated with powerful musculature of the buccal mass located at the center of the arm crown. Such bites do not always penetrate the skin of human beings (e.g. see p. 68 in Wells). ${ }^{150}$ The effects of penetrating bites are exacerbated by enzymes, venoms, other bioactive substances and microorganisms in the saliva. The possibility of injury should not be overlooked even when animals are transported in a plastic bag. ${ }^{464}$

The arms of all cephalopods are relatively strong, and this is especially the case in larger octopuses where the grip is enhanced by the numerous suckers. Areas of erythema may be induced if attempts are made to detach an animal by pulling in air, rather than allowing it to leave the arm naturally under water. Special precautions should be used if handling members of the teuthoid family as their arms/tentacles have hook-like appendages. Staff should be trained in how to remove animals that have become attached to their arms using the minimum of force and without inducing the animal to bite.

The use of Personal Protective Equipment (PPE), such as gloves or gauntlets resistant to penetration, may be suggested, but care should be taken to ensure that the gloves do not harm the delicate skin of cephalopods and that safe handling is not impaired by wearing gloves. In addition, the typical behaviour of the animals should be well recognised and mostly for signs of imminent aggression, escape attempt and other putative abnormal behaviours. 


\subsubsection{Chemical risk}

Direct contact with mucus, faeces and biological fluids. Biological materials can represent a risk of allergy, intolerance and/or toxicity particularly with repeated exposure. Exposure to mucus, faeces and ink may occur during routine cleaning and handling and other fluids during autopsy. Faeces and blood (haemolymph) are also potential routes of infection. We are not aware of reports of reactions to these biological fluids, but in case of doubt the use of waterproof gloves is recommended; in addition, in case of inadvertent contact with any biological substances, hands should be properly washed immediately.

Handlers and those undertaking autopsies should also be aware of any experimental procedures previously undertaken involving the administration of potentially hazardous substances (e.g. infectious agents, radioactive material, drugs, nanoparticles) to the animals so that assessment of potential risks can be undertaken.

Venom, enzymes and other pharmacologically active substances. The secretion from the posterior salivary glands of coleoid cephalopods (see Table 4.1 in Wells ${ }^{150}$ ) is injected into prey via the salivary papilla to immobilise and digest it with a mixture of venoms (i.e. cephalotoxin ${ }^{465}$ ), enzymes (e.g. chitinase, carboxypeptidase, hyaluronidase, phospholipase A2) and other biologically active substances (e.g. 5-hydroxytryptamine, dopamine, substance P) as reviewed by Ruder et al. ${ }^{466}$

A localised or systemic response could be induced by one or more of these substances particularly in sensitive individuals, but documented examples of systemic reactions to bites are rare except in the case of the blueringed octopus.

In fact, the venom of blue-ringed octopuses (Hapalochlaena spp.) can be fatal ${ }^{467}$ unless there is prompt medical attention. ${ }^{467,468}$ The toxin involved is the potent sodium channel blocker TTX $^{469}$ that is found in the posterior salivary glands, skin, branchial hearts, gills and Needham's sac, ${ }^{470}$ so care should also be taken with handling these animals post mortem. Recently, TTX has been found in the eggs with the levels increasing after laying: ${ }^{471}$ therefore, the risk with this species does not only come from adults. Other data show that the venom is produced by symbiotic bacteria (Aeromonas, Bacillus, Pseudomonas and Vibrio) found in the salivary glands. ${ }^{472}$ Clinically, the bite of the blueringed octopus is most often painless but freely bleeding. Erythema and edema at the bite site usually occurs, but the most important effects are those that are systemic. Most severe envenomations are characterised by generalised weakness, slurred speech, circumoral paraesthesias, respiratory difficulty and dysphagia. Such symptoms may last for $12-24$ hours. There is no antivenom, therefore treatment includes pressure immobilisation and immediate transport to a medical facility while monitoring respiratory and neurologic status. ${ }^{473}$

No reactions specifically attributable to the venoms from other species of cephalopod have been described, but there are several reports of localized reactions to bites from cephalopods although these seem rare. Wells reports having been bitten himself 10 or 20 times without event, but also describes a reaction to an $O$. vulgaris bite on the forearm in a student who had previously never been exposed to cephalopods. ${ }^{150}$ The response included swelling of the forearm (comparable to a bee sting) and overnight pain, both of which resolved the next day. A local skin reaction probably related to proteolytic activity has been described after red octopus $\left(O\right.$. rubescens) bite. ${ }^{474}$ Haemolytic activity against mammalian red cells has been reported in vitro with low concentrations of saliva from E. cirrhosa, ${ }^{475}$ but we are not aware of any evidence for haemolysis in vivo, although caution should be exerted.

Reactions to a bite will depend upon the sensitivity of the individual and this may be a particular issue with atopic individuals or those who have become sensitised by repeated exposure. The reaction to a bite may not be immediate as indicated by a case report of giant cellrich granulomatous dermatitis/panniculitis one month after a bite from an octopus (species not given) on the wrist. ${ }^{476}$ Bites are also a source of infection (see below) and again the reaction may be delayed.

Precautions should be the same as for the protection against bites (see above). All occurrences of bites by cephalopods should be recorded in the laboratory safety book, medical/paramedical advice sought particularly in cases where the skin is broken and the clinical outcome monitored. Anderson et al. reported anecdotally that immediate hot water treatment was effective in neutralising the localised effects of the bite of $O$. rubescens. ${ }^{474}$

Seawater and ink. Cephalopods in general and octopuses in particular can forcibly squirt seawater and/or ink directly at handlers. In addition to melanin, ink contains an array of bioactive substances including enzymes (e.g. tyrosinase, tyrosine hydroxylase) and other chemicals (e.g. dopamine, 5,6-dihydroxyindole). ${ }^{477,478}$ There is a theoretical risk that the seawater may be contaminated by pollutants or infectious agents in open systems but seawater or ink in the eye could cause irritation and also distract the handler from their task increasing the risk of a bite or animal escape. Wearing eye or face protection should be considered when handling cephalopods. 


\subsubsection{Biohazards}

The animals. Animals, especially those taken from the wild, can transmit infectious agents (zoonoses) to humans and as cephalopods host a number of bacteria $(\mathrm{Gram}+$ and -$)$, viruses and parasites; this is a potential risk for anyone in contact with cephalopods. The major risk of transmission is via accidental ingestion or a bite.

This section will not cover the potential pathogenicity of different agents, however, mention should be given to, for example, Anisakis and Aggregata that are known to be the cause of the zoonotic disease, as reviewed by Yang et al. ${ }^{479}$

Most information regarding infection with Anisakis comes from human consumption of uncooked cephalopods, but poor hand hygiene and laboratory practice especially when undertaking autopsies of fresh animals means that hand-to-mouth transmission is a possibility. An additional potential source of parasites (and possibly bacteria, viruses and toxins) is the fish, crustacea and molluscs used as food for cephalopods so wearing gloves and hand hygiene should also be considered.

The main documented risk is bacterial infection from bites although the bacteria may originate from contaminated water as well as the animal itself. ${ }^{480-482}$

Particular attention should be paid when handling cephalopods with skin lesions as these wounds are often infected (see section 4.4).

Seawater. Seawater itself is a potential reservoir and transmission vehicle for infectious organisms, chemical pollutants and toxins (e.g. from algae) particularly in open systems. In seawater, many pathogens can be responsible for infections, e.g. Staphylococcus aureus, Streptococcus pyogenes, Mycobacterium marinum, Vibrio vulnificus, Erysipelothrix rhusiopathiae, Aeromonas hydrophila, Pseudomonas aeruginosa, Prototheca wickerhamii. ${ }^{473}$

These represent a potential hazard for both animals and humans who have contact with the seawater but the risk should be minimal if the water quality is monitored and maintained within strict limits. Additional safety precautions may need to be put in place if there is a breakdown in water quality management especially for those who may need to decontaminate and clean the tanks.

Allergens. Repeated exposure to animals, chemicals (e.g. some disinfectants) and some disposables can result in the appearance of several clinical forms of allergic reaction (e.g. contact dermatitis or urticaria). To minimise the allergy risk operators should have a medical assessment before starting work and at regular intervals. Cephalopod mucus can be an irritant and has been occasionally described as an allergen (A. Affuso, pers. comm.). Since there are cases of an acquired allergy to cephalopod eggs, it is recommended that they are handled wearing gloves. Arginine kinase from Octopus fangsiao has been shown to react with $\operatorname{IgE}$ in the serum of octopus-allergenic subjects, ${ }^{483}$ emphasising the importance of identifying individuals who may be especially sensitive to cephalopods before they begin work so that the risk can be assessed and managed.

\subsection{A summary of practical advice}

9.3.1 Assess and manage the potential risks. Each person involved with cephalopods should be assessed, and monitored for potential risks, notes taken of the species involved and the work to be undertaken before any work assigned. For example, the risks in moving a potentially lethal blue-ringed octopus or a $30 \mathrm{~kg} \mathrm{E}$. dofleini between tanks are different from moving a $200 \mathrm{~g}$ cuttlefish. Protocols of good laboratory practice (GLP) - Standard Operating Procedures (SOPs), should be developed in conjunction with the Health and Safety officer or other person, incorporated into training programmes (see section 9) and common protocols (e.g. handling, cleaning, humane killing) displayed or kept in a file in the facility and in the room dedicated to procedures. Risk assessment will also need to comply with local 'out of hours' policies. All working areas should be equipped with required PPE (overalls, hypoallergenic gloves, safety glasses, eye wash) and have a telephone with an emergency number displayed.

9.3.2 Operator health. Most staff undergo some form of health assessment at the beginning of employment. Anyone working with cephalopods should be asked if they are allergic to these animals or if they are atopic so that risks can be managed. All staff with regular exposure to cephalopods should be monitored regularly for signs of sensitisation.

9.3.3 Recording incidents. All incidents (including 'near misses') should be recorded partly to facilitate development of improved protocols. For example, skin-penetrating bites must be recorded and reported immediately to the relevant person so that appropriate action can be taken and any delayed reactions (e.g. infections) documented.

The 'incident book' should be reviewed regularly and protocols and policies modified as required. Lessons for the wider cephalopod community should be posted on the CephRes web site (www.cephalopo dresearch.org) and if possible published.

9.3.4 Dealing with incidents. Staff should be familiar from training and laboratory SOPs/GLP with action to be taken in particular circumstances. The most likely incident requiring immediate action is a bite in which the skin is broken and the wound infiltrated with 
secretions from the posterior salivary glands and/or seawater. A protocol for this and other eventualities should be drawn up in consultation with the trained on-site first responder and a medical practitioner.

\section{Education and training: carers, researchers and veterinarians}

The Directive requires that all staff involved in regulated research are adequately educated and trained for tasks they are required to perform (Article 23) including: $i$. carrying out procedures on animals; $i$. designing procedures and projects; iii. taking care of animals; or $i v$. humane killing of animals.

Education and training therefore includes all staff involved in daily care (including veterinarians; since courses on marine invertebrate medicine are rare), researchers performing procedure and principal investigators designing studies. Anyone having direct contact with the animals will need to be able to demonstrate that they are practically competent in performing tasks or regulated procedures and have this competence assessed periodically. There may also be an argument for providing limited theoretical training for members of an institutional project/ethical review committee about novel species.

Trained individuals should understand and be able to demonstrate the importance of the animal welfare regulations and guidelines for housing, care and use, assessment of animal welfare including signs of illness, PSDLH and their palliation or treatment.

Developing training programmes to meet these requirements is a particular challenge for cephalopods as although knowledge of general care and welfare is relatively well developed (as reviewed in Fiorito et al. ${ }^{2}$ ), knowledge of PSDLH and their application to assessment of animal welfare aspects of a project are less well established. ${ }^{3,8}$ In addition, there are multiple species of cephalopod with a variety of care (see also Appendix 2) and welfare requirements. Many aspects of training will need to be delivered by animal technologists, researchers and veterinarians who have gained experience prior to the regulation of research involving cephalopods, but it would be desirable to involve some trainers with expertise in working with aquatic vertebrates (including expertise in ethical review).

Courses will need to align with specific national requirements, and will need to be recognised by the National Competent Authority (http://ec.europa.eu/ environment/chemicals/lab_animals/ms_en.htm) of Member States as fulfilling the requirements of the Directive 2010/63/EU for all persons involved in the use, care and breeding of cephalopods for scientific procedures. Recognition at EU level will facilitate movement of personnel between member states. The content and delivery of the modules should be validated by a university or other competent awarding body, and linked to Continuing Professional Development (CPD) programmes of professional bodies.

Training modules should be designed taking into account of the EU Commission working document of a development of a common education and training framework to fulfil the requirements under the Directive.

Ideally courses should be offered at EU level but as many aspects of training are 'hands-on' courses will need to be based in facilities with aquaria and access to several species. It is proposed that a training course should be structured into three modules; basic training, a speciesspecific module, procedures, PSDLH assessment and management module. These modules cover the key welfare assessment competencies and welfare training topics outlined in Tables 12 and 13 of Hawkins et al. ${ }^{44}$

Delivery and assessment of modules are not discussed here.

\subsection{Indicative content of modules}

10.1.1 Basic module: an introduction to cephalopods in research. This should cover: $i$. national and EU legislation on protection of animals used for scientific purposes; ii. a brief introduction to cephalopod biology; iii. why cephalopods were included in the Directive; $i v$. the philosophy of the institution regarding animal care and use; v. the requirement to comply with all national regulations and institutional guidelines; $v i$. the key differences between undertaking research in a legally regulated and an unregulated environment; vii. record keeping; viii. the requirement to respond immediately to any PSDLH issues; $i x$. reporting animal care and use concerns at institutional and national levels; $x$. health, safety, risk assessment and security; $x i$. roles of the institution and project ('ethical') review committee, veterinarian (or other competent expert), animal care, and research staff in the animal care and use programme; xii. public engagement.

10.1.2 Species-specific module. This focuses on the species utilised for research in a particular institution and should include the following species-specific topics: $i$. biology and behaviour; ii. supply, capture and transportation (including any additional permits and regulations); iii. environment (tanks, water, enrichment) and control; $i v$. signs of health, welfare and disease; $v$. assessing when an animal should be killed humanely; $v i$. specialised techniques for identification of individuals; vii. anaesthesia and humane killing; viii. tagging and marking; ix. genotyping; $x$. analgesia/anaesthesia/euthanasia/confirmation of death.

10.1.3 Procedures, PSDLH assessment and management module. This module has three main elements: 
a) Project/licence and ethical committee applications. This will cover: $i$. experimental design from a 3Rs perspective (see section 2.2.2; see also Smith et al. ${ }^{3}$ ); ii. principles of harm-benefit analysis (e.g. Bateson cube); iii. principles of severity assessment (prospective, actual and retrospective); $i v$. setting humane endpoints; $v$. writing a lay summary of the project/ethical application; vi. public engagement.

b) Recognising PSDLH and their management. This component deals with these aspects in depth as this module is intended for those designing projects or performing procedures some of which may never have been performed in a cephalopod previously. The following topics will be covered: $i$. evidence for the capacity of cephalopods to experience PSDLH (see review and discussion in Andrews et $a .^{8}$ ); ii. recognising PSDLH and techniques to minimise and treat them in the context of a regulated procedure; iii. special considerations regarding senescent cephalopods.

c) Procedures. This covers: $i$. what is a procedure within the meaning of the Directive?; $i i$. an introduction to basic surgical techniques and post-operative monitoring and care; iii. non-surgical procedures, techniques and assessing their impact on the animal.

d) Reporting studies under the Directive. Annual statistical return (Article 54) and an introduction to the ARRIVE Guidelines (www.nc3rs.uk/ ARRIVE; ${ }^{272}$ ) and the Gold Standard Publication Checklist. ${ }^{485,486}$

Education and training of all personnel involved in the research programme at whatever level is essential to ensure the optimal care and welfare of animals and for the standards to improve with time by identification and dissemination of examples of good practice. These Guidelines and recent publications ${ }^{1,2,487}$ establish the core material required for the delivery of the modules outlined here as a basis for education and training of personnel involved in research now regulated by the Directive. The next step will be to develop the above outlines into a document that can be used for accreditation of a course compliant with FELASA recommendations for laboratory animal science education and training, as outlined by Nevalainen et al. and recently updated. ${ }^{488,489}$

\section{Concluding comment}

This paper represents the first attempt by members of the international cephalopod community to develop guidelines for Care and Welfare of cephalopods utilised in scientific research. Although the guidelines primarily address the requirements of Directive 2010/63/EU, we anticipate they will also be utilised by the wider cephalopod research community outside the EU. It is recognised that in contrast to equivalent guidelines for vertebrates the evidence base for some aspects of these guidelines is not strong.

It is hoped that this paper will prompt research directed specifically at the Care and Welfare of cephalopods in the laboratory to provide a solid evidence base for future revisions of these guidelines.

\section{Disclaimer}

These Guidelines represent a consensus view, but inclusion in the authorship or contributor list does not necessarily imply agreement with all statements.

\section{Funding}

CephRes, AISAL and FELASA support this work. This publication is also supported by COST (European Cooperation in Science and Technology).

\section{Acknowledgements}

This work originates from a joint effort to develop the first Guidelines for the Care and Welfare of Cephalopods in Research according to Directive 2010/63/EU made by the Association for Cephalopod Research - CephRes, The Boyd Group (UK), and the Federation for Laboratory Animal Science Associations - FELASA. This has been initiated and subsequently developed following April 2012 CephRes Meeting in Vico Equense (Italy). We would like to thank Drs John Messenger, Andrew Packard and Sigurd von Boletsky for their contributions to the initial meeting where ideas for Guidelines for the Care and Welfare of Cephalopods were discussed. We are also grateful for comments and contributions by Piero Amodio (CephRes, Italy), and by Ariane Dröscher (University of Bologna, Italy) for historical data.

PA and GF conceived and edited this work as well as a making a major contribution to the authorship. GF and GP organised the meeting; GP and AC provided assistance to the leading authors on behalf of CephRes.

This manuscript provides the basis for the activities of the FA1301 COST Action 'A network for improvement of cephalopod welfare and husbandry in research, aquaculture and fisheries (CephsInAction; http://www.cost.eu/COST_Actions /fa/Actions/FA1301; www.cephsinaction.org)' aimed to facilitate and support the cephalopod community in meeting the challenges resulting from the inclusion of cephalopod molluscs in Directive 2010/63/EU.

We are grateful to Professor Torsten Wiesel for his enthusiasm and genuine interest for cephalopod biology research.

\section{References}

1. Ponte G, Dröscher A and Fiorito G. Fostering cephalopod biology research: past and current trends and topics. Invert Neurosci 2013; 13: 1-9. 
2. Fiorito G, Affuso A, Anderson DB, Basil J, Bonnaud L, Botta G, Cole A, D'Angelo L, De Girolamo P, Dennison N, Dickel L, Di Cosmo A, Di Cristo C, Gestal C, Fonseca R, Grasso F, Kristiansen T, Kuba M, Maffucci F, Manciocco A, Mark FK, Melillo D, Osorio D, Palumbo A, Perkins K, Ponte G, Raspa M, Shashar N, Smith J, Smith D, Sykes A, Villanueva R, Tublitz N, Zullo L and Andrews PLR. Cephalopods in neuroscience: Regulations, Research and the 3Rs. Invert Neurosci 2014; 14: 13-36.

3. Smith JA, Andrews PLR, Hawkins P, Louhimies S, Ponte $\mathrm{G}$ and Dickel L. Cephalopod research and EU Directive 2010/63/EU: Requirements, impacts and ethical review. J Exp Mar Biol Ecol 2013; 447: 31-45.

4. Jereb P, Roper CFE and Vecchione M. Introduction. Cephalopods of the World. An annotated and illustrated catalogue of species known to date. Volume 1. Chambered nautiluses and sepioids (Nautilidae, Sepiidae, Sepiolidae, Sepiadariidae, Idiosepiidae and Spirulidae). FAO Species Catalogue for Fishery Purposes. No. 4. Vol.1. Rome: FAO Fisheries Synopsis, 2005, pp. 1-13.

5. Roper CFE, Sweeney MJ and Nauen CE. FAO Species Catalogue, vol. 3. Cephalopods of the World. An Annotated and Illustrated Catalogue of Species of Interest to Fisheries. FAO Fisheries Synopsis, 1984, p. 277.

6. EFSA Panel on Animal Health and Welfare. Opinion of the Scientific Panel on Animal Health and Welfare (AHAW) on a request from the Commission related to the 'Aspects of the biology and welfare of animals used for experimental and other scientific purposes'. EFSA J 2005; 292: 1-136.

7. Crook RJ and Walters ET. Nociceptive behavior and physiology of molluscs: animal welfare implications. ILAR J 2011; 52: 185-195.

8. Andrews PLR, Darmaillacq AS, Dennison N, Gleadall IG, Hawkins P, Messenger JB, Osorio D, Smith VJ and Smith JA. The identification and management of pain, suffering and distress in cephalopods, including anesthesia, analgesia and humane killing. J Exp Mar Biol Ecol 2013; 447: 46-64.

9. Crook RJ, Lewis T, Hanlon RT and Walters ET. Peripheral injury induces long-term sensitization of defensive responses to visual and tactile stimuli in the squid Loligo pealeii, Lesueur 1821. J Exp Biol 2011; 214: 3173-3185.

10. Alupay JS, Hadjisolomou SP and Crook RJ. Arm injury produces long-term behavioral and neural hypersensitivity in octopus. Neurosci Lett 2013; 558: 137-142.

11. Crook RJ, Hanlon RT and Walters ET. Squid have nociceptors that display widespread Long-term sensitization and spontaneous activity after bodily injury. $J$ Neurosci 2013; 33: 10,021-10,026.

12. Young RE, Vecchione $M$ and Mangold KMM. Cephalopoda Cuvier 1797. Octopods, squids, nautiluses, etc. The Tree of Life Web Project 2012; http://tolweb.org/ Cephalopoda: Version 10 (2012, accessed 10 November 2014).

13. Home Office. Guidance on the Operation of the Animals (Scientific Procedures) Act 1986. https://www.gov.uk/ government/uploads/system/uploads/attachment_data/ file/291350/Guidance_on_the_Operation_of_ASPA.pdf London: TSO, 2014, p. 164.
14. European Commission. Implementation, interpretation and terminology of Directive 2010/63/EU-Q\&A document. Brussels: European Commission, 2014, p.49.

15. Shigeno S, Parnaik R and Ragsdale CW. Octopus Brains: The molecular architecture of functionally identified neural systems (Mollusca). J Morphol 2008; 269: 1466-1467.

16. Teichert C. Main features of cephalopod evolution. In: Clarke MR and Trueman ER (eds) The Mollusca. Paleontology and Neontology of Cephalopods. San Diego: Academic Press, Inc. 1988, pp.11-79.

17. Donovan DT. Cephalopod phylogeny and classification. Biol Rev 1964; 39: 259-287.

18. Young JZ. Evolution of the cephalopod brain. In: Clarke MR and Trueman ER (eds) The Mollusca. Vol. 12: Paleontology and neontology of cephalopods. San Diego, CA: Academic Press, 1988, pp.215-228.

19. Boletzky Sv. Cephalopod development and evolutionary concepts. In: Clarke MR and Trueman ER (eds) The Mollusca. Vol. 12: Paleontology and neontology of cephalopods. San Diego, CA: Academic Press, 1988, pp.185-202.

20. Clarke MR. Evolution of recent cephalopods-A brief review. In: Clarke MR and Trueman ER (eds) The Mollusca. Vol. 12: Paleontology and neontology of cephalopods. San Diego: Academic Press, Inc. 1988, pp.331-340.

21. Shigeno S, Sasaki T, Moritaki T, Kasugai T, Vecchione $\mathrm{M}$ and Agata K. Evolution of the cephalopod head complex by assembly of multiple molluscan body parts: Evidence from Nautilus embryonic development. J Morphol 2008; 269: 1-17.

22. Kroger B, Vinther $\mathbf{J}$ and Fuchs D. Cephalopod origin and evolution: A congruent picture emerging from fossils, development and molecules. Bioessays 2011; 33: 602-613.

23. Grasso FW and Basil JA. The evolution of flexible behavioral repertoires in cephalopod molluscs. Brain Behav Evol 2009; 74: 231-245.

24. Packard A. Cephalopods and fish: the limits of convergence. Biol Rev 1972; 47: 241-307.

25. Huffard CL. Cephalopod neurobiology: an introduction for biologists working in other model systems. Invert Neurosci 2013; 13: 11-18.

26. Borrelli L. Testing the contribution of relative brain size and learning capabilities on the evolution of Octopus vulgaris and other cephalopods. PhD Thesis, Stazione Zoologica Anton Dohrn, Italy \& Open University, UK, 2007.

27. Aronson RB. Ecology, paleobiology and evolutionary constraint in the octopus. Bull Mar Sci 1991; 49: 245-255.

28. Boyle PR. Cephalopod Life Cycles. London, UK: Academic Press, 1983, p.475.

29. Boyle PR and Rodhouse P. Cephalopods. Ecology and Fisheries. Oxford: Blackwell Science, 2005, p.452.

30. Trueman ER and Packard A. Motor performances of some cephalopods. J Exp Biol 1968; 49: 495-507.

31. Borrelli L, Gherardi F and Fiorito G. A Catalogue of Body Patterning in Cephalopoda. Napoli, Italy: Stazione Zoologica A. Dohrn; Firenze University Press, 2006, p.626. 
32. Packard A and Sanders G. What the octopus shows to the world. Endeavour 1969; 28: 92-99.

33. Hochner B. Octopuses. Curr Biol 2008; 18: R897-R898.

34. Zullo L and Hochner B. A new perspective on the organization of an invertebrate brain. Commun Integr Biol 2011; 4: 26-29.

35. Hochner B. An embodied view of octopus neurobiology. Curr Biol 2012; 22: R887-R892.

36. Borrelli L and Fiorito G. Behavioral analysis of learning and memory in cephalopods. In: Byrne JJ (ed.) Learning and Memory: A comprehensive reference. Oxford: Academic Press, 2008, pp.605-627.

37. Brown ER and Piscopo S. Synaptic plasticity in cephalopods; more than just learning and memory? Invert Neurosci 2013; 13: 35-44.

38. European Parliament and Council of the European Union. Directive 2010/63/EU of the European Parliament and of the Council of 22 September 2010 on the protection of animals used for scientific purposes. Strasbourg: Council of Europe, 2010.

39. Louhimies S. Revised EU Legislation on the Protection of Animals Used for Scientific Purposes Directive 2010/ 63/EU. J Shellfish Res 2011; 30: 1011.

40. Russell WMS and Burch RL. The Principles of Humane Experimental Technique. London: Methuen and Co. Ltd. 1959, p.238.

41. Anderson JA, Eijkholt M and Illes J. Ethical reproducibility: towards transparent reporting in biomedical research. Nature Methods 2013; 10: 843-845.

42. Blakemore C, MacArthur Clark J, Nevalainen T, Oberdorfer $M$ and Sussman A. Implementing the 3Rs in neuroscience research: A reasoned approach. Neuron 2012; 75: 948-950.

43. Hawkins D, Gallacher E and Gammell M. Statistical power, effect size and animal welfare: recommendations for good practice. Animal Welfare 2013; 22: 339-344.

44. van Luijk J, Cuijpers Y, van der Vaart L, Leenaars M and Ritskes-Hoitinga M. Assessing the search for information on Three Rs methods, and their subsequent implementation: A national survey among scientists in The Netherlands. Atla-Alternatives to Laboratory Animals 2011; 39: 429-447.

45. Bronstad A and Berg AGT. The role of organizational culture in compliance with the principles of the 3Rs. Lab Animal 2011; 40: 22-26.

46. European Commission and National Competent Authorities for the Implementation of Directive 2010/ 63/EU. Working document on a severity assessment framework. Brussels: European Commission, 2012, p.18.

47. European Commission. Examples to illustrate the process of severity classification, day-to-day assessment and actual severity assessment. Brussels: European Commission, 2013, p.52.

48. Animal Procedures Committee-APC. Review of CostBenefit Assessment in the Use of Animals in Research. London, UK: https://www.gov.uk/government/publica tions/review-of-cost-benefit-assessment-in-the-use-of-ani mals-in-research (accessed 10 November 2014), 2003.

49. Albertin CB, Bonnaud L, Brown CT, Crookes-Goodson WJ, da Fonseca RR, Di Cristo C, Dilkes BP, Edsinger-
Gonzales E, Freeman RM Jr., Hanlon RT, Koenig KM, Lindgren AR, Martindale MQ, Minx P, Moroz LL, Nodl MT, Nyholm SV, Ogura A, Pungor JR, Rosenthal JJ, Schwarz EM, Shigeno S, Strugnell JM, Wollesen T, Zhang $\mathrm{G}$ and Ragsdale CW. Cephalopod genomics: A plan of strategies and organization. Standards in Genomic Sciences 2012; 7: 175-188.

50. Iglesias J, Fuentes L and Villanueva R. Cephalopod Culture. Dordrecht: Springer Netherlands, 2014, p.494.

51. Braid HE. Systematics and ecology of the New Zealand Mastigoteuthidae (Cephalopoda, Oegopsida). MSc Thesis, AUT - Auckland University of Technology, New Zealand, 2013.

52. Rathjen WF. Cephalopod capture methods: an overview. Bull Mar Sci 1991; 49: 494-505.

53. Lane FW. Kingdom of the Octopus; the Life History of the Cephalopoda. New York: Sheridan House, 1960, p. 300 .

54. Lo Bianco S. Notizie biologiche riguardanti specialmente il periodo di maturità sessuale degli animali del Golfo di Napoli. Mitt Zool Stat Neapel 1909; 19: 513-763.

55. Chotiyaputta C. Cephalopod resources of Thailand. In: Okutani T and O'Dor RK (eds) Recent Advances in Cephalopod Fishery Biology. Tokyo: Tokai University Press, 1993, pp.71-80.

56. Grimpe G. Pflege, Behandlung und Zucht der Cephalopoden fur zoologische und physiologische Zwecke. In: Äberhalden E (ed.) Handbuch der biologischen Arbeitsmethoden. Berlin, Wien: Verlag Urban \& Schwarzenberg, 1928, pp.331-402.

57. Jones NJE, Ridgway ID and Richardson CA. Transport of cuttlefish, Sepia officinalis, eggs under dry and damp conditions. J Mollusc Stud 2009; 75: 192-194.

58. Berka R. The Transport of Live Fish: A Review. Rome: European Inland Fisheries Advisory Commission (EIFAC); FAO-Food and Agriculture Organization of the United Nations, 1986, p. 79.

59. Harmon TS. Methods for reducing stressors and maintaining water quality associated with live fish transport in tanks: a review of the basics. Rev Aquaculture 2009; 1: 58-66.

60. Lekang O-I. Transport of live fish. In: Lekang O-I (ed.) Aquaculture Engineering. Chichester, UK: John Wiley \& Sons, 2013, pp.328-338.

61. Sykes AV, Baptista FD, Gonçalves RA and Andrade JP. Directive $2010 / 63 /$ EU on animal welfare: a review on the existing scientific knowledge and implications in cephalopod aquaculture research. Rev Aquaculture 2012; 4: $142-162$.

62. Gutfreund Y, Flash T, Yarom Y, Fiorito G, Segev I and Hochner B. Organization of octopus arm movements: a model system for studying the control of flexible arms. J Neurosci 1996; 16: 7297-7307.

63. Gutfreund Y, Flash T, Fiorito $G$ and Hochner B. Patterns of arm muscle activation involved in octopus reaching movements. J Neurosci 1998; 18: 5976-5987.

64. Johnson SK. Transport of fish and crustaceans in sealed containers. Inland Aquaculture Handbook ed. College Station, TX, USA: Texas Aquaculture Association, 1988, p. A1504-A1509. 
65. Swann L. Transportation of Fish in Bags. University of Missoury, MU Extension: http://extension.missouri.edu/ p/MX392 (accessed 10 November 2014), 1993.

66. Slater $\mathrm{M}$ and Buttling O. Giant Pacific Octopus Husbandry Manual; Enteroctopus dofleini in Public aquaria. London, UK: BIAZA-The British and Irish Association for Zoos and Aquariums, 2011, p.31.

67. Bower JR, Sakurai Y, Yamamoto J and Ishii H. Transport of the ommastrephid squid Todarodes pacificus under cold-water anesthesia. Aquaculture 1999; 170: 127-130.

68. Gleadall IG. Low dosage of magnesium sulphate as a long-term sedative during transport of firefly squid, Watasenia scintillans. J Exp Mar Biol Ecol 2013; 447: 138-139.

69. Sanders GD. The cephalopods. In: Corning WC, Dyal JA and Willows AOD (eds) Invertebrate Learning. Vol. 3, Cephalopods and Echinoderms. New York, N.Y.: Plenum Press, 1975, pp.1-101.

70. Darmaillacq A-S, Chichery R, Poirier R and Dickel L. Effect of early feeding experience on subsequent prey preference by cuttlefish, Sepia officinalis. Dev Psychobiol 2004; 45: 239-244.

71. Maldonado H. The positive learning process in Octopus vulgaris. Z Vgl Physiol 1963; 47: 191-214.

72. Boycott BB. Learning in Octopus vulgaris and other cephalopods. Pubbl Staz Zool Napoli 1954; 25: 67-93.

73. Castellanos-Martinez S and Gestal C. Pathogens and immune response of cephalopods. J Exp Mar Biol Ecol 2013; 447: 14-22.

74. Toonen R. Aquarium invertebrates: Housing an octopus. Advanced Aquarist (online) 2003; 2.

75. Hanlon RT. Aspects of the biology of the squid, Loligo (Doryteuthis) plei in captivity. PhD, University of Miami, USA, 1978.

76. Yang WT, Hanlon RT, Lee PG and Turk PE. Design and function of closed seawater systems for culturing loliginid squids. Aquac Eng 1989; 8: 47-65.

77. Hanlon RT, Hixon RF and Hulet WH. Survival, growth, and behavior of the loliginid squids Loligo plei, Loligo pealei, and Lolliguncula brevis (Mollusca, Cephalopoda) in Closed Sea-Water Systems. Biol Bull 1983; 165: 637-685.

78. Gutnick T, Richter J, Hochner B and Kuba MJ. Inland closed system laboratories chances and challenges. J Shellfish Res 2011; 30: 1005-1006.

79. Huguenin JE and Colt J. Design and Operating Guide for Aquaculture Seawater Systems, 2nd ed. Amsterdam: Elsevier Science, 2002, p.328.

80. Lekang O-I. Aquaculture Engineering, 2nd ed. Oxford: Wiley-Blackwell, 2013, p.432.

81. Winterstein $H$. Über die chemische Regulierung der Atmung bei den Cephalopoden. Z Vgl Physiol 1924; 2: 315-328.

82. Borer KT and Lane CE. Oxygen requirements of Octopus briareus Robson at different temperatures and oxygen concentrations. J Exp Mar Biol Ecol 1971; 7: 263-269.

83. Wells MJ and Wells J. The control of ventilatory and cardiac responses to changes in ambient oxygen tension and oxygen demand in octopus. J Exp Biol 1995; 198: $1717-1727$.

84. Katsanevakis S, Stephanopoulou S, Miliou H, MoraitouApostolopoulou M and Verriopoulos G. Oxygen consumption and ammonia excretion of Octopus vulgaris (Cephalopoda) in relation to body mass and temperature. Mar Biol 2005; 146: 725-732.

85. Houlihan DF, Duthie G, Smith PJ, Wells MJ and Wells $\mathrm{J}$. Ventilation and circulation during exercise in Octopus vulgaris. J Comp Physiol 1986; 156: 683-689.

86. Wells MJ and Smith PJS. The performance of the Octopus circulatory system: A triumph of engineering over design. Experientia 1987; 43: 487-499.

87. Melzner F, Mark FC and Portner HO. Role of bloodoxygen transport in thermal tolerance of the cuttlefish, Sepia officinalis. Integr Comp Biol 2007; 47: 645-655.

88. Gutowska MA, Portner HO and Melzner F. Growth and calcification in the cephalopod Sepia officinalis under elevated seawater $\mathrm{pCO}_{2}$. Mar Ecol Progr Ser 2008; 373: 303-309.

89. Repolho T, Baptista M, Pimentel MS, Dionisio G, Truebenbach K, Lopes VM, Lopes AR, Calado R, Diniz $\mathrm{M}$ and Rosa R. Developmental and physiological challenges of octopus (Octopus vulgaris) early life stages under ocean warming. J Comp Physiol B 2014; 184: 55-64.

90. Kaplan MB, Mooney TA, McCorkle DC and Cohen AL. Adverse effects of ocean acidification on early development of squid (Doryteuthis pealeii). PLOS ONE 2013; 8: e63714.

91. Rosa R, Trübenbach K, Pimentel MS, Boavida-Portugal J, Faleiro F, Baptista M, Dionísio G, Calado R, Põrtner HO and Repolho T. Differential impacts of ocean acidification and warming on winter and summer progeny of a coastal squid (Loligo vulgaris). J Exp Biol 2014; 217 : $518-525$.

92. Gutowska MA and Melzner F. Abiotic conditions in cephalopod (Sepia officinalis) eggs: embryonic development at low $\mathrm{pH}$ and high $\mathrm{pCO}_{2}$. Mar Biol 2009; 156: 515-519.

93. Gutowska MA, Melzner F, Langenbuch M, Bock C, Claireaux G and Portner HO. Acid-base regulatory ability of the cephalopod (Sepia officinalis) in response to environmental hypercapnia. J Comp Physiol B: Biochem System Environ Physiol 2010; 180: 323-335.

94. Gutowska MA, Melzner F, Portner HO and Meier S. Cuttlebone calcification increases during exposure to elevated seawater $\mathrm{pCO}(2)$ in the cephalopod Sepia officinalis. Mar Biol 2010; 157: 1653-1663.

95. Hu MY, Tseng YC, Stumpp M, Gutowska MA, Kiko R, Lucassen $\mathrm{M}$ and Melzner F. Elevated seawater PCO2 differentially affects branchial acid-base transporters over the course of development in the cephalopod Sepia officinalis. Am J Physiol Regul Integr Comp Physiol 2011; 300: R1100-R1114.

96. Hu MY, Tseng YC, Stumpp M, Gutowska MA, Kiko R, Lucassen $M$ and Melzner F. Elevated seawater PCO2 differentially affects branchial acid-base transporters over the course of development in the cephalopod Sepia 
officinalis (Correction; vol 300, pg R1100, 2011). Am J Physiol Regul Integr Comp Physiol 2011; 301: R559.

97. Lange GD and Hartline PH. Retinal responses in squid and octopus. J Comp Physiol 1974; 93: 19-36.

98. Houck BA. Temporal spacing in the activity patterns of three Hawaiian shallow-water octopus. Nautilus 1982; 96: 152-156.

99. Hormiga JA, Almansa E, Sykes AV and Torres NV. Model based optimization of feeding regimens in aquaculture: Application to the improvement of Octopus vulgaris viability in captivity. $J$ Biotechnol 2010; 149: 209-214.

100. Meisel DV, Byrne RA, Kuba M, Griebel U and Mather JA. Circadian rhythms in Octopus vulgaris. Berliner Paläobiol Abh 2003; 3: 171-177.

101. Brown ER, Piscopo S, De Stefano R and Giuditta A. Brain and behavioural evidence for rest-activity cycles in Octopus vulgaris. Behav Brain Res 2006; 172: 355-359.

102. Frank MG, Waldrop RH, Dumoulin M, Aton S and Boal JG. A preliminary analysis of sleep-like states in the cuttlefish Sepia officinalis. PLoS ONE 2012; 7: 6: e38125.

103. Meisel DV, Byrne RA, Kuba M, Mather J, Ploberger W and Reschenhofer E. Contrasting activity patterns of two related octopus species, Octopus macropus and Octopus vulgaris. J Comp Psychol 2006; 120: 191-197.

104. Fiorito G, von Planta C and Scotto P. Problem solving ability of Octopus vulgaris Lamarck (Mollusca, Cephalopoda). Behav Neural Biol 1990; 53: 217-230.

105. Sykes A, Domingues PM, Márquez L and Andrade JP. The effects of tank colours on the growth and survival of cuttlefish (Sepia officinalis, Linnaeus 1758) hatchlings and juveniles. Aquac Res 2011; 42: 441-449.

106. Sykes A, Pereira D, Rodriguez C, Lorenzo A and Andrade JP. Effects of increased tank bottom areas on cuttlefish (Sepia officinalis, L.) reproduction performance. Aquac Res 2013; 44: 1017-1028.

107. DeTolla LJ, Srinivas S, Whitaker BR, Andrews C, Hecker B, Kane AS and Reimschuessel R. Guidelines for the care and use of fish in research. ILAR $J 1995 ; 37$ : 159-173.

108. Boletzky Sv and Hanlon RT. A review of the laboratory maintenance, rearing and culture of cephalopod molluscs. Mem Natl Mus Victoria 1983; 44: 147-187.

109. Karlsen HE, Packard A and Sand O. Cephalopods are definitely not deaf. J Physiol Lond 1989; 415: 75P.

110. Komak S, Boal JG, Dickel L and Budelmann BU. Behavioural responses of juvenile cuttlefish (Sepia officinalis) to local water movements. Mar Freshwater Behav Physiol 2005; 38: 117-125.

111. Mooney TA, Hanlon RT, Christensen-Dalsgaard J, Madsen PT, Ketten DR and Nachtigall PE. Sound detection by the longfin squid (Loligo pealeii) studied with auditory evoked potentials: sensitivity to low-frequency particle motion and not pressure. J Exp Biol 2010; 213: 3748-3759.

112. Samson JE, Mooney TA, Gussekloo SWS and Hanlon RT. Behavioral responses to sound stimuli in cuttlefish (Sepia officinalis). Integr Comp Biol 2013; 53: E188.
113. Mooney TA, Hanlon R, Madsen P, ChristensenDalsgaard J, Ketten D and Nachtigall P. Potential for sound sensitivity in cephalopods. In: Popper AN and Hawkins A (eds) The Effects of Noise on Aquatic Life. Advances in Experimental Medicine and Biology (v 730). New York: Springer, 2012, pp.125-128.

114. Kaifu K, Akamatsu T and Segawa S. Underwater sound detection by cephalopod statocyst. Fish Sci 2008; 74: 781-786.

115. Packard A, Karlsen HE and Sand O. Low-frequency hearing in cephalopods. J Comp Physiol A 1990; 166: 501-505.

116. André M, Solé M, Lenoir M, Durfort M, Quero C, Mas A, Lombarte A, van der Schaar M, López-Bejar M, Morell M, Zaugg S and Houégnigan L. Low-frequency sounds induce acoustic trauma in cephalopods. Front Ecol Environ 2011; 9: 489-493.

117. Fewtrell JL and McCauley RD. Impact of air gun noise on the behaviour of marine fish and squid. Marine Poll Bull 2012; 64: 984-993.

118. Guerra A, Martinell X, lez AF, Vecchione M, Gracia J and Martinell J. A new noise detected in the ocean. J Mar Biol Ass UK 2007; 87: 1255-1256.

119. Committee for the Update of the Guide for the Care and Use of Laboratory Animals. Guide for the Care and use of Laboratory Animals. Eighth Edition. Washington, DC: The National Academy Press, 2011, p.248.

120. Strathmann MF. Reproduction and Development of Marine Invertebrates of the Northern Pacific Coast. Data and Methods for the Study of Eggs, Embryos and Larvae. Seattle, WA: University of Washington Press, 1987, p.682.

121. Huffard CL, Caldwell RL, DeLoach N, Gentry DW, Humann P, MacDonald B, Moore B, Ross R, Uno T and Wong $\mathrm{S}$. Individually unique body color patterns in octopus (Wunderpus photogenicus) allow for photoidentification. PLoS ONE 2008; 3.

122. Byrne RA, Wood JB, Anderson RC, Griebel U and Mather JA. Non-invasive methods of identifying and tracking wild squid. Ferrantia 2010; 59: 22-31.

123. Petrella V. Identificazione e marcatura di Octopus vulgaris (Mollusca, Cephalopoda): stato delle conoscenze e studio di fattibilità. Università degli Studi di Napoli 'Federico II'. Facoltà di Scienze Matematiche, Fisiche e Naturali, 2003.

124. Zeeh KM and Wood JB. Impact of visible implant elastomer tags on the growth rate of captive Caribbean reef squid Sepioteuthis sepioidea. Fish Res 2009; 95: 362-364.

125. Brewer RS and Norcross BL. Long-term retention of internal elastomer tags in a wild population of North Pacific giant octopus (Enteroctopus dofleini). Fish Res 2012; 134-136: 17-20.

126. Malham SK, Runham RW and Secombes CJ. Lysozyme and antiprotease activity in the lesser octopus Eledone cirrhosa (Lam.) (Cephalopoda). Dev Comp Immunol 1998; 22: 27-37.

127. Replinger SE and Wood JB. A preliminary investigation of the use of subcutaneous tagging in Caribbean reef squid Sepioteuthis sepioidea (Cephalopoda: Loliginidae). Fish Res 2007; 84: 308-313. 
128. Fuentes L, Otero JJ, Sánchez FJ and Iglesias J. Application of different external tagging methods to Octopus vulgaris Cuvier, 1797, with special reference to T-bar anchor tags and Petersen disks. Bol Inst Esp Oceanogr 2006; 22: 3-11.

129. Sims DW, Humphries NE, McHugh M and Wearmouth VJ. Electronic tagging of cuttlefish Sepia officinalis and octopus Eledone cirrhosa to study commonalities in behaviour patterns and comparisons with marine fish. J Shellfish Res 2011; 30: 1020-1021.

130. Bloor ISM, Wearmouth VJ, Cotterell SP, Mchugh MJ, Humphries NE, Jackson EL, Attrill MJ and Sims DW. Movements and behaviour of European common cuttlefish Sepia officinalis in English Channel inshore waters: First results from acoustic telemetry. J Exp Mar Biol Ecol 2013; 448: 19-27.

131. Estefanell J, Socorro J, Afonso JM, Roo J, FernandezPalacios $\mathrm{H}$ and Izquierdo MS. Evaluation of two anaesthetic agents and the passive integrated transponder tagging system in Octopus vulgaris (Cuvier 1797). Aquac Res 2011; 42: 399-406.

132. Wearmouth VJ, Durkin OC, Bloor I, McHugh M, Rundle $\mathrm{J}$ and Sims DW. A method for long-term electronic tagging and tracking of juvenile and adult European common cuttlefish Sepia officinalis. J Exp Mar Biol Ecol 2013; 447: 149-155.

133. Byrne RA, Griebel U, Wood JB and Mather JA. Squid say it with skin: a graphic model for skin displays in Caribbean reef squid (Sepioteuthis sepioidea). Berliner Paläobiol Abh 2003; 3: 29-35.

134. Hanlon RT and Messenger JB. Cephalopod Behaviour. Cambridge: Cambridge University Press, 1996, p.232.

135. Budelmann BU. Active marine predators: the sensory world of Cephalopods. Mar Freshwater Behav Physiol 1996; 27: 59-75.

136. Rodhouse PG and Nigmatullin CM. Role as consumers. Philos Trans $R$ Soc Lond B 1996; 351: 1003-1022.

137. Hastein T, Scarfe AD and Lund VL. Science-based assessment of welfare: aquatic animals. Rev Sci Tech Off Int Epiz 2005; 24: 529-547.

138. Mather JA. Enrichment and cephalopods. Enrichment Record 2012; Summer: 24-28.

139. Westermann B, Ruth P, Litzlbauer HD, Beck I, Beuerlein K, Schmidtberg H, Kaleta EF and Schipp R. The digestive tract of Nautilus pompilius (Cephalopoda, Tetrabranchiata): an X-ray analytical and computational tomography study on the living animal. J Exp Biol 2002; 205: 1617-1624.

140. O’Dor RK, Mangold K, Boucherrodoni R, Wells MJ and Wells J. Nutrient absorption, storage and remobilization in Octopus vulgaris. Mar Behav Physiol 1984; 11: 239-258.

141. Boucher-Rodoni R, Boucaud-Camou E and Mangold K. Feeding and digestion. Cephalopod Life Cycles. London: Academic Press London, 1987, pp.85-108.

142. Nixon $M$ and Young JZ. The Brains and Lives of Cephalopods. Oxford: Oxford University Press, 2003, p.392.

143. Ohkouchi N, Tsuda R, Chikaraishi Y and Tanabe K. A preliminary estimate of the trophic position of the deep- water ram's horn squid Spirula spirula based on the nitrogen isotopic composition of amino acids. Mar Biol 2013; 160: 773-779.

144. Lee PG. Nutrition of cephalopods: Fueling the system. Mar Freshwater Behav Physiol 1995; 25: 35-51.

145. Oestmann DJ, Scimeca JM, Forsythe J, Hanlon R and Lee P. Special considerations for keeping cephalopods in laboratory facilities. Contemp Top Lab Anim Sci 1997; 36: 89-93.

146. Domingues P, Sykes A, Sommerfield A, Almansa E, Lorenzo A and Andrade JP. Growth and survival of cuttlefish (Sepia officinalis) of different ages fed crustaceans and fish. Effects of frozen and live prey. Aquaculture 2004; 229: 239-254.

147. Ferreira A, Marquez L, Almansa E, Andrade JP, Lorenzo A and Domingues PM. The use of alternative diets to culture juvenile cuttlefish, Sepia officinalis: effects on growth and lipid composition. Aquac Nutr 2010; 16: 262-275.

148. Mangold K and Bidder AM. L'appareil digestif et la digestion. In: Grassé PP and Mangold K (eds) Cephalopodes. Traite de Zoologie. Paris: Masson, 1989, pp.321-373.

149. Andrews PLR and Tansey EM. The Digestive Tract of Octopus vulgaris. The anatomy, physiology and pharmacology of the upper tract. J Mar Biol Ass UK 1983; 63: 109-134.

150. Wells MJ. Octopus: Physiology and Behaviour of an Advanced Invertebrate. London: Chapman and Hall, 1978, p.417.

151. Domingues PM, DiMarco FP, Andrade JP and Lee PG. Effect of artificial diets on growth, survival and condition of adult cuttlefish, Sepia officinalis Linnaeus, 1758. Aquacult Int 2005; 13: 423-440.

152. Nixon M and Mangold K. The early life of Sepia officinalis, and the contrast with that of Octopus vulgaris (Cephalopoda). J Zool 1998; 245: 407-421.

153. Boletzky Sv. Biology of early life stages in cephalopod molluscs. Adv Mar Biol 2003; 44: 143-203.

154. Hanlon RT, Bidwell JP and Tait R. Strontium is required for statolith development and thus normal swimming behavior of hatchling cephalopods. J Exp Biol 1989; 141: 187-195.

155. Villanueva R and Bustamante P. Composition in essential and non-essential elements of early stages of cephalopods and dietary effects on the elemental profiles of Octopus vulgaris paralarvae. Aquaculture 2006; 261: 225-240.

156. Madan JJ and Wells MJ. Cutaneous respiration in Octopus vulgaris. J Exp Biol 1996; 199: 2477-2483.

157. Vaz-Pires P, Seixas P and Barbosa A. Aquaculture potential of the common octopus (Octopus vulgaris Cuvier, 1797): a review. Aquaculture 2004; 238: 221-238.

158. Packard A. The skin of Cephalopods (Coleoids): general and special adaptations. In: Trueman ER and Clarke MR (eds) The Mollusca, Vol. 11. Form and Function. New York: Academic Press, 1988, pp.37-67.

159. Walker JJ, Longo $\mathrm{N}$ and Bitterman ME. Octopus in laboratory-handling, maintenance, training. Behav Res Meth Instrum 1970; 2: 15-18. 
160. Williamson R. A sensory basis for orientation in cephalopods. J Mar Biol Ass UK 1995; 75: 83-92.

161. Griffin G. Evaluating environmental enrichment is essential. Enrichment Record 2012; Summer: 29-33.

162. Maple TL and Perdue BM. Environmental enrichment. Zoo Animal Welfare. 14 ed. Springer Berlin Heidelberg, 2013, pp. 95-117.

163. Bayne K and Würbel H. The impact of environmental enrichment on the outcome variability and scientific validity of laboratory animal studies. Rev sci tech Off int Epiz 2014; 33: 273-280.

164. Shepherdson DJ, Mellen JD and Hutchins M. Second Nature: Environmental enrichment for captive animals. Washington: Smithsonian Institution Press, 1998, p.350.

165. Baumans V and Van Loo PLP. How to improve housing conditions of laboratory animals: The possibilities of environmental refinement. Vet $J$ 2013; 195: 24-32.

166. Conceicao LEC, Yufera M, Makridis P, Morais S and Dinis MT. Live feeds for early stages of fish rearing. Aquac Res 2010; 41: 613-640.

167. Kistler C, Hegglin D, Wurbel $\mathrm{H}$ and Konig B. Preference for structured environment in zebrafish (Danio rerio) and checker barbs (Puntius oligolepis). Appl Anim Behav Sci 2011; 135: 318-327.

168. Williams TD, Readman GD and Owen SF. Key issues concerning environmental enrichment for laboratoryheld fish species. Lab Anim 2009; 43: 107-120.

169. Wood JB and Wood DA. Enrichment for an advanced invertebrate. The Shape of Enrichment 1999; 8: 1-5.

170. Cenni F, Parisi G and Gherardi F. Effects of habitat complexity on the aggressive behaviour of the American lobster (Homarus americanus) in captivity. Appl Anim Behav Sci 2010; 122: 63-70.

171. Yasumuro $\mathrm{H}$ and Ikeda $\mathrm{Y}$. Effects of environmental enrichment on the behavior of the tropical octopus Callistoctopus aspilosomatis. Mar Freshwater Behav Physiol 2011; 44: 143-157.

172. Poirier R, Chichery R and Dickel L. Effects of rearing conditions on sand digging efficiency in juvenile cuttlefish. Behav Proc 2004; 67: 273-279.

173. Dickel L, Darmaillacq AS, Poirier R, Agin V, Bellanger $\mathrm{C}$ and Chichery R. Behavioural and neural maturation in the cuttlefish Sepia officinalis. Vie Milieu 2006; 56: 89-95.

174. Anderson RC and Wood JB. Enrichment for giant Pacific octopuses: Happy as a clam? J Appl Anim Welfare Sci 2001; 4: 157-168.

175. Boal JG. Behavioral research methods for octopuses and cuttlefishes. Vie et Milieu-Life and Environment 2011; 61: 203-210.

176. Dunstan AJ, Ward PD and Marshall NJ. Vertical distribution and migration patterns of Nautilus pompilius. PLOS ONE 2011; 6 .

177. Crook RJ and Basil JA. Flexible spatial orientation and navigational strategies in chambered nautilus. Ethology 2013; 119: 77-85.

178. Delbeek JC. Husbandry of the big-fin reef squid (Sepioteuthis lessoniana) at the Waikiki Aquarium. Drum and Croaker 2002; 33: 35-37.
179. Amodio P, Andrews PLR, Salemme M, Ponte G and Fiorito $\mathrm{G}$. The use of artificial crabs for testing predatory behavior and health in the octopus. AltexAlternatives to Animal Experimentation 2014; doi: http://dx.doi.org/10.14573/altex.1401282: 1-12.

180. Agin V, Chichery R, Maubert E and Chichery MP. Time-dependent effects of cycloheximide on long-term memory in the cuttlefish. Pharmacol Biochem Behav 2003; 75: 141-146.

181. Colmers WF, Hixon RF, Hanlon RT, Forsythe JW, Ackerson MV, Wiederhold ML and Hulet WH. Spinner cephalopods-defects of statocyst suprastructures in an invertebrate analog of the vestibular apparatus. Cell Tissue Res 1984; 236: 505-515.

182. Boyle PR. Ventilation rate and arousal in the octopus. $J$ Exp Mar Biol Ecol 1983; 69: 129-136.

183. Wells MJ, O'Dor RK, Mangold K and Wells J. Diurnal changes in activity and metabolic rate in Octopus vulgaris. Mar Behav Physiol 1983; 9: 275-287.

184. Mather JA. Daytime activity of juvenile Octopus vulgaris in Bermuda. Malacologia 1988; 29: 69-76.

185. Aitken JP, O'Dor RK and Jackson GD. The secret life of the giant Australian cuttlefish Sepia apama (Cephalopoda): Behaviour and energetics in nature revealed through radio acoustic positioning and telemetry (RAPT). J Exp Mar Biol Ecol 2005; 320: 77-91.

186. Mather JA, Resler S and Cosgrove J. Activity and movement patterns of Octopus dofleini. Mar Behav Physiol 1985; 11: 301-314.

187. Mather JA. How do octopuses use their arms? J Comp Psychol 1998; 112: 306-316.

188. Saunders WB and Landman NH. Nautilus. The Biology and Paleobiology of a Living Fossil. New York: Plenum Press, 1987, p.632.

189. Polglase JL, Bullock AM and Roberts RJ. Wound-healing and the hemocyte response in the skin of the lesser octopus Eledone cirrhosa (Mollusca, Cephalopoda). J Zool 1983; 201: 185-204.

190. Tressler J, Maddox F, Goodwin E, Zhang Z and Tublitz N. Arm regeneration in two species of cuttlefish Sepia officinalis and Sepia pharaonis. Inv Neurosci 2013; 14: $37-49$.

191. Aristotle. Historia Animalium, English translation by D'Arcy Wenthworth Thompson. Volume IV. The works of Aristotle translated into English under the editorship of J.A. Smith and W.D. Ross Oxford: Clarendon Press, 1910.

192. Hochner B, Shomrat T and Fiorito G. The octopus: a model for a comparative analysis of the evolution of learning and memory mechanisms. Biol Bull 2006; 210: 308-317.

193. Anderson RC, Mather JA, Monette MQ and Zimsen SRM. Octopuses (Enteroctopus dofleini) recognize individual humans. J Appl Anim Welfare Sci 2010; 13: 261-272.

194. Tricarico E, Borrelli L, Gherardi F and Fiorito G. I know my neighbour: individual recognition in Octopus vulgaris. PLoS ONE 2011; 6.

195. Tricarico E, Amodio P, Ponte G and Fiorito G. Cognition and recognition in the cephalopod mollusc Octopus vulgaris: Coordinating interaction with 
environment and conspecifics. In: Witzany G (ed.) Biocommunication of Animals. Dordrecht: Springer Science + Business Media, 2014, pp.337-349.

196. Wood JB, Pennoyer KE and Derby CD. Ink is a conspecific alarm cue in the Caribbean reef squid, Sepioteuthis sepioidea. J Exp Mar Biol Ecol 2008; 367: 11-16.

197. Bennett H and Toll RB. Intramantle inking: A stress behavior in Octopus bimaculoides (Mollusca: Cephalopoda). JAALAS 2011; 50: 943-945.

198. Garcia-Franco M. Anaesthetics for the squid Sepioteuthis sepioidea (Mollusca: Cephalopoda). Comp Biochem Physiol C Comp Pharmacol 1992; 103: 121-123.

199. Stern RM, Koch KL and Andrews PLR. NauseaMechanisms and Management. Oxford: Oxford University Press, 2011, p.480.

200. Packard A. Through the looking-glass of cephalopod colour patterns. In: Alleva E, Fasolo A, Lipp H-P, Nadel L and Ricceri L (eds) Behavioural Brain Research in Naturalistic and Semi-Naturalistic Settings. Dordrecht: Kluwer Academic, 1995, pp.105-130.

201. Messenger JB. Cephalopod chromatophores: neurobiology and natural history. Biol Rev 2001; 76: 473-528.

202. Packard A. Organization of cephalopod chromatophore systems: a neuromuscular image-generator. In: Abbott NJ, Williamson R and Maddock L (eds) Cephalopod Neurobiology. Oxford: Oxford University Press, 1995, pp.331-367.

203. Moltschaniwskyj N, Hall K, Marian J, Nishiguchi M, Sakai M, Shulman D, Sinclair B, Sinn D, Staudinger M, Van Gelderen R, Villanueva R and Warnke K. Ethical and welfare considerations when using cephalopods as experimental animals. Rev Fish Biol Fisheries 2007; 17: 455-476.

204. Josef N, Amodio P, Fiorito G and Shashar N. Camouflaging in a complex environment. Octopuses use specific features of their surroundings for background matching. PLoS ONE 2012; 7: e37579.

205. Mäthger LM and Hanlon RT. Malleable skin coloration in cephalopods: selective reflectance, transmission and absorbance of light by chromatophores and iridophores. Cell Tissue Res 2007; 329: 179-186.

206. Mäthger LM, Denton EJ, Marshall NJ and Hanlon RT. Mechanisms and behavioural functions of structural coloration in cephalopods. J R Soc Interface 2009; 6: S149-S163.

207. Mäthger LM, Roberts SB and Hanlon RT. Evidence for distributed light sensing in the skin of cuttlefish, Sepia officinalis. Biol Letters 2010; 6: 600-603.

208. Mooney TA, Lee WJ and Hanlon RT. Long-duration anesthetization of squid (Doryteuthis pealeii). Mar Freshwater Behav Physiol 2010; 43: 297-303.

209. Packard A and Hochberg FG. Skin patterning in Octopus and Other Genera. Symp Zool Soc Lond 1977; 38: 191-231.

210. Bullock AM, Polglase JL and Phillips SE. The wound healing and haemocyte response in the skin of the lesser octopus Eledone cirrhosa (Mollusca: Cephalopoda) in the presence of Vibrio tubiashii. J Zool 1987; 211: 373-385.

211. Hanley JS, Shashar N, Smolowitz R, Mebane W and Hanlon RT. Soft-sided tanks improve long-term health of cultured cuttlefish. Biol Bull 1999; 197: 237-238.

212. Boyle PR. Methods for the aquarium maintenance of the common octopus of British Waters, Eledone cirrhosa. Lab Anim 1981; 15: 327-331.

213. Hurley AC, Lange GD and Hartline PH. Adjustable pinhole camera eye of Nautilus. J Exp Zool 1978; 205: $37-43$.

214. Douglas RH, Williamson R and Wagner HJ. The pupillary response of cephalopods. J Exp Biol 2005; 208: 261-265.

215. McCormick LR and Cohen JH. Pupil light reflex in the Atlantic brief squid, Lolliguncula brevis. J Exp Biol 2012; 215: 2677-2683.

216. Beer T. Die Akkomodation des Kephalopodenauge. Arch f d gesamte Physiol 1897; 67: 541-586.

217. Magnus R. Die Pupillarreaktion von Oktopoden. Arch $f$ d gesamte Physiol 1902; 92: 623-643.

218. Messenger JB. Optomotor responses and nystagmus in intact, blinded and statocystless cuttlefish (Sepia officinalis L.). J Exp Biol 1970; 53: 789-796.

219. Budelmann BU, Schipp R and Boletzky Sv. Cephalopoda. In: Harrison FW and Kohn AJ (eds) Microscopic Anatomy of Invertebrates. New York: Wiley-Liss, Inc, 1997, pp.119-414.

220. Williamson R. Efferent activity in the octopus statocyst nerves. J Comp Physiol A 1986; 158: 125-132.

221. Carlson B. Collection and aquarium maintenance of Nautilus. In: Saunders WB and Landman NH (eds) Nautilus: The biology and paleobiology of a living fossil. New York: Plenum Press, 1987, pp.563-577.

222. Neumeister H and Budelmann BU. Structure and function of the Nautilus statocyst. Philos Trans R Soc Lond B 1997; 352: 1565-1588.

223. Barord GJ, Ju CH and Basil JA. First report of a successful treatment of a mucodegenerative disease in the chambered nautilus (Nautilus pompilius). J Zoo Wildl Med 2012; 43: 636-639.

224. Budelmann BU and Young JZ. The statocyst-oculomotor system of Octopus vulgaris: Extraocular eye muscles, eye muscle nerves, statocyst nerves and the oculomotor centre in the central nervous system. Philos Trans $R$ Soc Lond B 1984; 306: 159-189.

225. Nixon M. Changes in body weight and intake of food by Octopus vulgaris. J Zool 1966; 150: 1-9.

226. Gestal C, de la Cadena MP and Pascual S. Malabsorption syndrome observed in the common octopus Octopus vulgaris infected with Aggregata octopiana (Protista: Apicomplexa). Dis Aquat Org 2002; 51: 61-65.

227. Caldarone EM, MacLean SA and Sharack B. Evaluation of bioelectrical impedance analysis and Fulton's condition factor as nonlethal techniques for estimating short-term responses in postsmolt Atlantic salmon (Salmo solar) to food availability. Fish Bull 2012; 110: 257-270.

228. Engelhard GH, Blanchard JL, Pinnegar JK, van der Kooij J, Bell ED, Mackinson S and Righton DA. 
Body condition of predatory fishes linked to the availability of sandeels. Mar Biol 2013; 160: 299-308.

229. Kotrschal A, Fischer B and Taborsky B. A noninvasive method to determine fat content in small fish based on swim bladder size estimation. J Exp Zool 2011; 315: 408-415.

230. Ranney SH, Fincel MJ, Wuellner MR, VanDeHey JA and Brown ML. Assessing length-related bias and the need for data standardization in the development of standard weight equations. N Am J Fish Manag 2010; 30: $655-664$.

231. Rennie MD and Verdon R. Development and evaluation of condition indices for the lake whitefish. $N \mathrm{Am} \mathrm{J}$ Fish Manag 2008; 28: 1270-1293.

232. Cade BS, Terrell JW and Porath MT. Estimating fish body condition with quantile regression. $N$ Am J Fish Manag 2008; 28: 349-359.

233. Bentley KT and Schindler DE. Body condition correlates with instantaneous growth in stream-dwelling Rainbow Trout and Arctic Grayling. Trans Am Fisher Soc 2013; 142: 747-755.

234. Green AJ. Mass/length residuals: Measures of body condition or generators of spurious results? Ecology 2001; 82: 1473-1483.

235. Labocha MK and Hayes JP. Morphometric indices of body condition in birds: a review. J Ornithol 2012; 153: $1-22$.

236. Bidder AM. Evidence for an absorptive function of the 'liver' of Octopus vulgaris Lam. Pubbl Staz Zool Napoli 1957; 29: 139-150.

237. Saunders WB. Natural rates of growth and longevity of Nautilus belauensis. Paleobiology 1983; 9: 280-288.

238. Forsythe JW and Van Heukelem WF. Growth. In: Boyle PR (ed.) Cephalopod Life Cycles. Comparative Reviews. London: Academic Press, 1987, pp.135-156.

239. Joll LM. Growth and food-intake of Octopus tetricus (Mollusca-Cephalopoda) in Aquaria. Aust J Mar Fresh Res 1977; 28: 45-56.

240. Smith PJS, Duthie GG, Wells MJ and Houlihan DF. Continuous recording of arterial blood $\mathrm{pO}_{2}$ in Octopus vulgaris during hypoxia and movement. J Exp Biol 1985; 117: 475-479.

241. Wells MJ, Hanlon RT, Lee PG and DiMarco FP. Respiratory and cardiac performance in Lolliguncula brevis (Cephalopoda, Myopsida): the effects of activity, temperature and hypoxia. J Exp Biol 1988; 138: 17-36.

242. Boal JG and Ni JN. Ventilation rate of cuttlefish, Sepia officinalis, in response to visual stimuli. Veliger 1996; 39 : 342-347.

243. Andrews PLR and Tansey EM. The effects of some anesthetic agents in Octopus vulgaris. Comp Biochem Physiol 1981; 70C: 241-247.

244. King AJ, Henderson SM, Schmidt MH, Cole AG and Adamo SA. Using ultrasound to understand vascular and mantle contributions to venous return in the cephalopod Sepia officinalis L. J Exp Biol 2005; 208: 2071-2082.

245. King AJ and Adamo SA. The ventilatory, cardiac and behavioural responses of resting cuttlefish (Sepia officinalis L.) to sudden visual stimuli. $J$ Exp Biol 2006; 209: 1101-1111.

246. Grimaldi AM, Agnisola C and Fiorito G. Using ultrasound to estimate brain size in the cephalopod Octopus vulgaris Cuvier in vivo. Brain Res 2007; 1183: 66-73.

247. Margheri L, Ponte G, Mazzolai B, Laschi C and Fiorito G. Non-invasive study of Octopus vulgaris arm morphology using ultrasound. $J$ Exp Biol 2011; 214: 3727-3731.

248. Tateno S. Non-invasive analysis of mantle movements in Octopus vulgaris. In: Okutani T, O'Dor RK and Kubodera $\mathrm{T}$ (eds) Recent Advances in Fisheries Biology. Tokyo: Tokai University Press, 1993, pp.559-569.

249. Collins AJ and Nyholm SV. Obtaining hemocytes from the Hawaiian Bobtail Squid Euprymna scolopes and observing their adherence to symbiotic and non-symbiotic bacteria. JoVE 2010; e1714.

250. Malham SK, Coulson CL and Runham NW. Effects of repeated sampling on the haemocytes and haemolymph of Eledone cirrhosa (Lam.). Comp Biochem Physiol Part A 1998; 121: 431-440.

251. Locatello L, Fiorito G, Finos L and Rasotto MB. Behavioural and immunological responses to an immune challenge in Octopus vulgaris. Physiol Behav 2013; 122: 93-99.

252. Malham SK and Runham NW. A brief review of the immunology of Eledone cirrhosa. S Afr J Mar Sci 1998; 20: $385-391$.

253. Rocca E and Ghiretti F. Ricerche sulle emocianine, VII: Sulla capacità dell'emocianina di Octopus vulgaris di legare il biossido di carbonio. Boll Soc It Biol Sper 1963; 39: 2075-2077.

254. Henze M. Zur Kenntnis des Hämocyanins. Z physiol Chem 1901; 33: 370-384.

255. Malham SK, Lacoste A, Gelebart F, Cueff A and Poulet SA. A first insight into stress-induced neuroendocrine and immune changes in the octopus Eledone cirrhosa. Aquat Living Resour 2002; 15: 187-192.

256. Henze M. Zur Kenntnis des Hämocyanins. 2. Mitteilung. Z physiol Chem 1904; 43: 290-298.

257. Henze M. Chemische Untersuchungen an Octopoden. $Z$ physiol Chem 1908; 55: 433-444.

258. Gestal C, Guerra A and Pascual S. Aggregata octopiana (Protista: Apicomplexa): a dangerous pathogen during commercial Octopus vulgaris ongrowing. ICES J Mar Sci 2007; 64: 1743-1748.

259. Yin F, Sun P, Peng S, Tang B, Zhang D, Wang C, Mu C and Shi $\mathrm{Z}$. The respiration, excretion and biochemical response of the juvenile common Chinese cuttlefish, Sepiella maindroni at different temperatures. Aquaculture 2013; 402-403: 127-132.

260. Long SM, Ryder KJ and Holdway DA. The use of respiratory enzymes as biomarkers of petroleum hydrocarbon exposure in Mytilus edulis planulatus. Ecotoxicol Environ Saf 2003; 55: 261-270.

261. Ramasamy MS and Murugan A. Potential antimicrobial activity of marine molluscs from Tuticorin, Southeast Coast of India against 40 biofilm bacteria. $J$ Shellfish Res 2005; 24: 243-251. 
262. Castillo MG, Goodson MS and McFall-Ngai MJ. Identification and molecular characterization of a complement C3 molecule in a lophotrochozoan, the Hawaiian bobtail squid Euprymna scolopes. Dev Comp Immunol 2009; 33: 69-76.

263. Alpuche J, Pereyra A, Mendoza-Hernandez G, Agundis C, Rosas C and Zenteno E. Purification and partial characterization of an agglutinin from Octopus maya serum. Comp Biochem Physiol Part B Biochem Mol Biol 2010; 156: 1-5.

264. Ramasamy P, Subhapradha N, Srinivasan A, Shanmugam V, Krishnamoorthy J and Shanmugam A. In vitro evaluation of antimicrobial activity of methanolic extract from selected species of cephalopods on clinical isolates. Afr J Microbiol Res 2011; 5: 3884-3889.

265. Collins AJ, LaBarre BA, Won BSW, Shah MV, Heng S, Choudhury MH, Haydar SA, Santiago J and Nyholm SV. Diversity and partitioning of bacterial populations within the accessory nidamental gland of the squid Euprymna scolopes. Appl Environ Microb 2012; 78: 4200-4208.

266. Nithya M, Ambikapathy V and Panneerselvam A. Effect of pharaoh's cuttlefish ink against bacterial pathogens. Asian J Plant Sci Res 2011; 1: 49-55.

267. Larson SE and Anderson RC. Fecal hormones measured within giant pacific octopuses Enteroctopus dofleini. J Aquat Anim Health 2010; 22: 152-157.

268. Storebakken T, Kvien IS, Shearer KD, GrisdaleHelland B, Helland SJ and Berge GM. The apparent digestibility of diets containing fish meal, soybean meal or bacterial meal fed to Atlantic salmon (Salmo salar): evaluation of different faecal collection methods. Aquaculture 1998; 169: 195-210.

269. Refstie S, Storebakken T and Roem AJ. Feed consumption and conversion in Atlantic salmon (Salmo salar) fed diets with fish meal, extracted soybean meal or soybean meal with reduced content of oligosaccharides, trypsin inhibitors, lectins and soya antigens. Aquaculture 1998; 162: 301-312.

270. Corse E, Costedoat C, Chappaz R, Pech N, Martin JF and Gilles A. A PCR-based method for diet analysis in freshwater organisms using $18 \mathrm{~S}$ rDNA barcoding on faeces. Mol Ecol Resour 2010; 10: 96-108.

271. Dobell C. The life-history and chromosome cycle of Aggregata eberthi (Protozoa: Sporozoa: Coccidia). Parasitology 1925; 17: 1-136.

272. Kilkenny C, Browne WJ, Cuthill IC, Emerson M and Altman DG. Improving Bioscience Research Reporting: The ARRIVE Guidelines for Reporting Animal Research. PLoS Biology 2010; 8: e1000412.

273. Canali E, Ponte G, Belcari P, Rocha F and Fiorito G. Evaluating age in Octopus vulgaris: estimation, validation and seasonal differences. Mar Ecol Progr Ser 2011; 441: 141-149.

274. Forsythe JW. A working hypothesis on how seasonal temperature change may impact the field growth of young cephalopods. In: Okutani T, O'Dor RK and Kubodera $\mathrm{T}$ (eds) Recent Advances in Cephalopod Fisheries Biology. Tokyo: Tokai University Press, 1993, pp.133-143.
275. Jackson GD. Cephalopod growth: historical context and future directions. Mar Freshwater Res 2004; 55: 327-329.

276. Moltschaniwskyj NA. Understanding the process of growth in cephalopods. Mar Freshwater Res 2004; 55: 379-386.

277. Rocha F, Guerra A and Gonzalez AF. A review of reproductive strategies in cephalopods. Biol Rev 2001; 76: 291-304.

278. Wodinsky J. Hormonal inhibition of feeding and death in octopus - control by optic gland secretion. Science 1977; 198: 948-951.

279. Meisel DV, Byrne RA, Mather JA and Kuba M. Behavioral sleep in Octopus vulgaris. Vie Milieu 2011; 61: 185-190.

280. Anderson RC, Wood JB and Byrne RA. Octopus senescence: the beginning of the end. J Appl Anim Welfare Sci 2002; 5: 275-283.

281. Reimschuessel R and Stoskopf MK. Octopus automutilation syndrome. J Invert Pathol 1990; 55: 394-400.

282. Harms CA, Lewbart GA, McAlarney R, Christian LS, Geissler K and Lemons C. Surgical excision of mycotic (Cladosporium sp.) granulomas from the mantle of a cuttlefish (Sepia officinalis). J Zoo Wildl Med 2006; 37: 524-530.

283. van Heukelem WF. Laboratory maintenance, breeding, rearing, and biomedical-research potential of Yucatan octopus (Octopus maya). Lab Anim Sci 1977; 27: 852-859.

284. Hermosilla CA, Rocha F, Fiorito G, Gonzalez AF and Guerra A. Age validation in common octopus Octopus vulgaris using stylet increment analysis. ICES J Mar Sci 2010; 67: 1458-1463.

285. Jackson GD. Age and growth of the tropical nearshore loliginid squid Sepioteuthis lessoniana determined from statolith growth-ring analysis. Fish Bull 1990; 88: 113-118.

286. Jackson GD and Moltschaniwskyj NA. The influence of ration level on growth and statolith increment width of the tropical squid Sepioteuthis lessoniana (Cephalopoda: Loliginidae): an experimental approach. Mar Biol 2001; 138: 819-825.

287. Jackson GD. Advances in defining the life histories of myopsid squid. Mar Freshwater Res 2004; 55: 357-365.

288. Arkhipkin AI. Statoliths as 'black boxes' (life recorders) in squid. Mar Freshwater Res 2005; 56: 573-583.

289. Arkhipkin AI and Shcherbich ZN. Thirty years' progress in age determination of squid using statoliths. J Mar Biol Ass UK 2012; 92: 1389-1398.

290. Bettencourt V, Coelho L, Andrade JP and Guerra A. Age and growth of the squid Loligo vulgaris off the south coast of Portugal, using statolith analysis. J Mollusc Stud 1996; 62: 359-366.

291. Bettencourt V and Guerra A. Age studies based on daily growth increments in statoliths and growth lamellae in cuttlebone of cultured Sepia officinalis. Mar Biol 2001; 139: 327-334.

292. Ceriola L and Jackson GD. Growth, hatch size and maturation in a southern population of the loliginid 
squid Loliolus noctiluca. J Mar Biol Ass UK 2010; 90: 755-767.

293. Challier L, Royer J and Robin JP. Variability in age-atrecruitment and early growth in English Channel Sepia officinalis described with statolith analysis. Aquat Living Resour 2002; 15: 303-311.

294. Choe S. Daily age markings on the shell of cuttlefishes. Nature 1963; 197: 306-307.

295. Jackson GD and Moltschaniwskyj NA. Analysis of precision in statolith derived age estimates of the tropical squid Photololigo (Cephalopoda: Loliginidae). ICES J Mar Sci 1999; 56: 221-227.

296. Jackson GD, Wotherspoon S and McGrath-Steer BL. Temporal population dynamics in arrow squid Nototodarus gouldi in southern Australian waters. Mar Biol 2005; 146: 975-983.

297. Martínez P, Bettencourt V, Guerra A and Moltschaniwskyj NA. How temperature influences muscle and cuttlebone growth in juvenile cuttlefish (Sepia elliptica) (Mollusca: Cephalopoda) under conditions of food stress. Can J Zool 2000; 78: 1855-1861.

298. Perez JAA, Odor RK, Beck $P$ and Dawe EG. Evaluation of gladius dorsal surface structure for age and growth studies of the short-finned squid, Illex illecebrosus (Teuthoidea: Ommastrephidae). Can J Fish Aquat Sci 1996; 53: 2837-2846.

299. Perez JAA, de Aguiar DC and dos Santos JAT. Gladius and statolith as tools for age and growth studies of the squid Loligo plei (Teuthida: Loliginidae) off southern Brazil. Braz Arch Biol Technol 2006; 49: 747-755.

300. Raya CP, Fernandez-Nunez M, Balguerias E and Hernandez-Gonzalez CL. Progress towards aging cuttlefish Sepia hierredda from the Northwestern African Coast using statoliths. Mar Ecol Progr Ser 1994; 114: 139-147.

301. Sakai J and Matsumoto JJ. Proteolytic enzymes of squid mantle muscle. Comp Biochem Physiol B 1981; 68: 389-395.

302. Hurtado JL, Borderías J, Montero P and Haejung AN. Characterization of Proteolytic Activity in octopus (Octopus vulgaris) arm muscle. J Food Biochem 1999; 23: 469-483.

303. Owen R. Memoir on the Pearly Nautilus (Nautilus Pompilius, Linn.). London: Richard Taylor, 1832, p.68.

304. Tompsett DH. Sepia. Liverpool: The University Press of Liverpool, 1939, p.184.

305. Williams LW. The Anatomy of the Common Squid Loligo pealii, Lesueur. Leiden, Holland: E.J. Brill, 1909, p.92.

306. Chapko MK, Grossbeck ML, Hansen RL, Maher TD, Middleton RS and Simpson RW. Devilfish. A Practical Guide to the Dissection of Octopus. Wayne Senior High School, 1962, p. 65.

307. Isgrove A. Eledone. London: Williams and Norgate, 1909.

308. Mather JA and Anderson RC. Ethics and invertebrates: a cephalopod perspective. Dis Aquat Org 2007; 75: 119-129.

309. Mather JA. Cephalopod consciousness: Behavioural evidence. Conscious Cogn 2008; 17: 37-48.
310. Mydlarz LD, Jones LE and Harvell CD. Innate immunity environmental drivers and disease ecology of marine and freshwater invertebrates. Ann Rev Ecol Evol Syst 2006; 37: 251-288.

311. Murata M, Ishi M and Osako M. On the regeneration of tentacle of the oceanic squid, Ommastrephes bartrami (Lesueur). Bull Hokkaido Reg Fish Res Lab 1981; 46: $1-14$.

312. Bush SL. Economy of arm autotomy in the mesopelagic squid Octopoteuthis deletron. Marine Ecology Progress Series 2012; 458: 133-140.

313. Wells MJ. Brain and Behaviour in Cephalopods. London: Heinemann, 1962, p.171.

314. Boyle PR. The UFAW Handbook on the Care and Management of Cephalopods in the Laboratory. Potters Bar, Herts, UK: Universities Federation for Animal Welfare, 1991, p.63.

315. Gestal C, Nigmatullin CM, Hochberg FG, Guerra A and Pascual S. Aggregata andresi n. sp (Apicomplexa: Aggregatidae) from the ommastrephid squid Martialia hyadesi in the SW Atlantic Ocean and some general remarks on Aggregata spp. in cephalopod hosts. Syst Parasitol 2005; 60: 65-73.

316. Mladineo I and Jozic M. Aggregata infection in the common octopus, Octopus vulgaris (Linnaeus 1758), Cephalopoda: Octopodidae, reared in a flow-through system. Acta Adriat 2005; 46: 193-199.

317. Longshaw M, Feist SW and Bateman KS. Parasites and pathogens of the endosymbiotic pea crab (Pinnotheres pisum) from blue mussels (Mytilus edulis) in England. J Invert Pathol 2012; 109: 235-242.

318. Shields JD. Diseases of spiny lobsters: A review. J Invert Pathol 2011; 106: 79-91.

319. Bonami JR and Zhang SY. Viral diseases in commercially exploited crabs: A review. J Invert Pathol 2011; 106: 6-17.

320. Nixon M and Budelmann BU. Scale worms: Occasional food of octopus. J Mollusc Stud 1984; 50: 39-42.

321. Loker ES, Adema CM, Zhang SM and Kepler TB. Invertebrate immune systems - not homogeneous, not simple, not well understood. Immunol Rev 2004; 198: $10-24$.

322. Litman GW and Dishaw LJ. Histocompatibility: Clarifying fusion confusion. Curr Biol 2013; 23: R934-R935.

323. Ford LA. Host defense mechanisms of cephalopods. Annu Rev Fish Dis 1992; 2: 25-41.

324. Novoa B, Tafalla C, Guerra A and Figueras A. Cellular immunological parameters of the octopus, Octopus vulgaris. J Shellfish Res 2002; 21: 243-248.

325. Rodriguez-Dominguez H, Soto-Bua M, Iglesias-Blanco R, Crespo-Gonzalez C, Axias-Fernandez C and GarciaEstevez J. Preliminary study on the phagocytic ability of Octopus vulgaris Cuvier, 1797 (Mollusca: Cephalopoda) haemocytes in vitro. Aquaculture 2006; 254: 563-570.

326. Cowden RR and Curtis SK. Cephalopods. In: Ratcliffe NA and Rowley AF (eds) Invertebrate Blood Cells. Vol. 1: General aspects, animals without true circulatory systems to cephalopods. London, UK: Academic Press, 1981, pp.301-323. 
327. Stuart AE. The reticulo-endothelial apparatus of the lesser octopus, Eledone cirrosa. J Pathol Bacteriol 1968; 96: 401-412.

328. Dales RP. Defence of invertebrates against bacterial infection. J R Soc Med 1979; 72: 688-696.

329. Castellanos-Martinez S, Prado-Alvarez M, Lobo-daCunha A, Azevedo C and Gestal C. Morphologic, cytometric and functional characterization of the common octopus (Octopus vulgaris) hemocytes. Dev Comp Immunol 2014; 44: 50-58.

330. Rogener W, Renwrantz L and Uhlenbruck G. Isolation and characterization of a lectin from the hemolymph of the cephalopod Octopus vulgaris (Lam) inhibited by alpha-D-lactose and N-acetyl-lactosamine. Dev Comp Immunol 1985; 9: 605-616.

331. Fisher WS and Dinuzzo AR. Agglutination of bacteria and erythrocytes by serum from six species of marine mollusks. J Invert Pathol 1991; 57: 380-394.

332. Thogersen IB, Salvesen G, Brucato FH, Pizzo SV and Enghild JJ. Purification and characterization of an alpha-macroglobulin proteinase-inhibitor from the mollusk Octopus vulgaris. Biochem J 1992; 285: 521-527.

333. Wommack KE and Colwell RR. Virioplankton: Viruses in aquatic ecosystems. Microbiol Mol Biol Rev 2000; 64: 69-114.

334. Rohwer F and Thurber RV. Viruses manipulate the marine environment. Nature 2009; 459: 207-212.

335. Breitbart M. Marine viruses: Truth or dare. Ann Rev Mar Sci 2012; 4: 425-448.

336. Hanlon RT and Forsythe JW. Diseases of Mollusca: Cephalopoda. Diseases caused by microorganisms. In: Kinne O (ed.) Diseases of Marine Animals. Volume III-Introduction, Cephalopoda, Annelida, Crustacea, Chaetognatha, Echinodermata, Urochordata. Hamburg, Germany: Biologische Anstalt, Helgoland, 1990, pp. $23-46$.

337. Rungger D, Rastelli M, Braendle E and Malsberg Rg. Viruslike particle associated with lesions in muscles of Octopus vulgaris. J Invert Pathol 1971; 17: 72-80.

338. Devauchelle G and Vago C. Particules d'allure virale dans les cellules de l'estomac de la seiche, Sepia officinalis L. (Mollusques, Cephalopodes). C R Hebd Seances Acad Sci 1971; 272: 894-896.

339. Budelmann BU. Autophagy in octopus. S Afr J Mar Sci 1998; 20: 101-108.

340. Farley CA. Viruses and viruslike lesions in marine mollusks. Mar Fish Rev 1978; 40: 18-20.

341. Gregory CR, Latimer KS, Pennick KE, Benson K and Moore T. Novel iridovirus in a nautilus (Nautilus spp.). $J$ Vet Diagn Invest 2006; 18: 208-211.

342. Gomez DK, Mori K, Okinaka Y, Nakai T and Park SC. Trash fish can be a source of betanodaviruses for cultured marine fish. Aquaculture 2010; 302: 158-163.

343. Vendramin N, Patarnello P, Toffan A, Panzarin V, Cappellozza E, Tedesco P, Terlizzi A, Terregino C and Cattoli G. Viral encephalopathy and retinopathy in groupers (Epinephelus spp.) in southern Italy: a threat for wild endangered species? BMC Vet Res 2013; 9: doi:10.1186/1746-6148-9-20.
344. Vanni A, Fichi G, Cardeti G, Cersini A, Perrucci S, Lenzi F, DeWolf T, Fronte B, Ricci E, Campeis F and Susini F. Potenziali patogeni in popolazione naturale e in soggetti stabulati di Octopus vulgaris. Atti Società Italiana di Patologia Ittica, XIX Convegno Nazionale 2013; 80 .

345. Morioka S, Sakata T, Tamaki A, Shioji T, Funaki A, Yamamoto $Y$, Naka $H$, Terasoma $F$, Imai $K$ and Matsuo K. A food-borne norovirus outbreak at a primary school in Wakayama prefecture. Jpn J Infect Dis 2006; 59: 205-207.

346. Karagiannis I, Detsis M, Gkolfinopoulou K, Pervanidou D, Panagiotopoulos T and Bonovas S. An outbreak of gastroenteritis linked to seafood consumption in a remote Northern Aegean island, FebruaryMarch 2010. Rural and Remote Health 2010; 10: 1507.

347. McCarthy KS, Guntapong R, Thattiyaphong A, Wangroongsarb P, Hall AJ, Olsen SJ and Holtz TH. Outbreak of norovirus gastroenteritis infection, Thailand. Southeast Asian J Trop Med Public Health 2013; 44: 409-416.

348. Gomez DK, Baeck GW, Kim JH, Choresca $\mathrm{CH}$ and Park SC. Genetic analysis of betanodaviruses in subclinically infected aquarium fish and invertebrates. Curr Microbiol 2008; 56: 499-504.

349. Gomez DK, Baeck GW, Kim JH, Choresca CH and Park SC. Molecular detection of betanodaviruses from apparently healthy wild marine invertebrates. J Invert Pathol 2008; 97: 197-202.

350. Tanaka S, Aoki H and Nakai T. Pathogenicity of the nodavirus detected from diseased sevenband grouper Epinephelus septemfasciatus. Fish Pathol 1998; 33: 31-36.

351. Scimeca JM. Cephalopods. In: Lewbart GA (ed.) Invertebrate Medicine. Ames, Iowa: Blackwell Publishing, 2006, pp.79-89.

352. McFall-Ngai MJ. Animal-bacterial interactions in the early life history of marine invertebrates: The Euprymna scolopes-Vibrio fischeri Symbiosis. Am Zool 1994; 34: 554-561.

353. Nyholm SV and McFall-Ngai MJ. The winnowing: establishing the squid-vibrio symbiosis. Nature Rev Microbiol 2004; 2: 632-642.

354. Pernice M, Wetzel S, Gros O, Boucher-Rodoni R and Dubilier N. Enigmatic dual symbiosis in the excretory organ of Nautilus macromphalus (Cephalopoda: Nautiloidea). Proc $R$ Soc Lond $B$ 2007; 274: 1143-1152.

355. Ford LA, Alexander SK, Cooper KM and Hanlon RT. Bacterial populations of normal and ulcerated mantle tissue of the squid, Lolliguncula brevis. J Invert Pathol 1986; 48: 13-26.

356. Sherrill J, Spelman LH, Reidel CL and Montali RJ. Common cuttlefish (Sepia officinalis) mortality at the National Zoological Park: Implications for clinical management. J Zoo Wildl Med 2000; 31: 523-531.

357. Hanlon RT, Forsythe JW, Cooper KM, Dinuzzo AR, Folse DS and Kelly MT. Fatal penetrating skin ulcers in laboratory-reared octopuses. J Invert Pathol 1984; 44: 67-83. 
358. Forsythe JW, Hanlon RT and Lee PG. A formulary for treating cephalopod mollusc diseases. In: Cheng TC and Perkins FO (eds) Pathology in Marine Aquaculture. New York: Academic Press, 1990, pp.51-63.

359. Gestal C, Abollo E and Pascual S. Rickettsiales-like organisms in the gills of reared Octopus vulgaris Mollusca, Cephalopoda. Bull Eur Assoc Fish Pathol 1998; 181: 13-14.

360. Farto R, Armada SP, Montes M, Guisande JA, Perez MJ and Nieto TP. Vibrio lentus associated with diseased wild octopus (Octopus vulgaris). J Invert Pathol 2003; 83: 149-156.

361. Hanlon RT, Yang WT, Turk PE, Lee PG and Hixon RF. Laboratory culture and estimated life span of the Eastern Atlantic squid, Loligo forbesi Steenstrup, 1856 (Mollusca: Cephalopoda). Aquac Res 1989; 20: 15-34.

362. Polglase JL. A preliminary report on the Thraustochytrid(s) and Labyrinthulid(s) associated with a pathological condition in the lesser octopus Eledone cirrhosa. Bot Mar 1980; 23: 699-706.

363. Gestal C, Belcari P, Abollo E and Pascual S. Parasites of cephalopods in the northern Tyrrhenian Sea (western Mediterranean): new host records and host specificity. Sci Mar 1999; 63: 39-43.

364. Pascual S and Hochberg FG. Marine parasites as biological tags of cephalopod hosts. Parasitol Today 1996; 12: 324-327.

365. Pascual S, Gestal C, Estevez JM, Rodriguez H, Soto M, Abollo E and Arias C. Parasites in commerciallyexploited cephalopods (Mollusca, Cephalopoda) in Spain: An updated perspective. Aquaculture 1996; 142: $1-10$.

366. Pascual S and Guerra A. Vexing question on fisheries research: the study of cephalopods and their parasites. Iberus 2001; 19: 87-95.

367. González AF, Pascual S, Gestal C, Abollo E and Guerra A. What makes a cephalopod a suitable host for parasite? The case of Galician waters. Fish Res 2003; 60: 177-183.

368. Pascual S, Gonzalez A and Guerra A. Parasites and cephalopod fisheries uncertainty: towards a waterfall understanding. Rev Fish Biol Fisheries 2007; 17: 635.

369. Overstreet RM and Hochberg FG. Digenetic trematodes in cephalopods. J Mar Biol Ass UK 1975; 55: 893-910.

370. Hochberg FG. Diseases of Mollusca: Cephalopoda. Diseases caused by Protistans and Metazoans. In: Kinne O (ed.) Diseases of Marine Animals. Volume III-Introduction, Cephalopoda, Annelida, Crustacea, Chaetognatha, Echinodermata, Urochordata. Hamburg, Germany: Biologische Anstalt, Helgoland, 1990, pp.47-202.

371. Furuya H, Ota M, Kimura R and Tsuneki K. Renal organs of cephalopods: A habitat for dicyemids and chromidinids. J Morphol 2004; 262: 629-643.

372. Furuya H, Hochberg FG and Tsuneki K. Cell number and cellular composition in infusoriform larvae of dicyemid mesozoans (Phylum Dicyemida). Zool Sci 2004; 21: $877-889$.
373. Abollo E, Gestal C, Lopez A, González AF, Guerra A and Pascual S. Squid as trophic bridges for parasite flow within marine ecosystems: The case of Anisakis simplex (Nematoda: Anisakidae), or when the wrong way can be right. S Afr J Mar Sci 1998; 20: 223-232.

374. Lehnert K, Seibel H, Hasselmeier I, Wohlsein P, Iversen M, Nielsen NH, Heide-Jörgensen MP, PrengerBerninghoff $\mathrm{E}$ and Siebert $\mathrm{U}$. Increase in parasite burden and associated pathology in harbour porpoises (Phocoena phocoena) in West Greenland. Polar Biol 2013; 1-11.

375. Santoro M, Mattiucci S, Work T, Cimmaruta R, Nardi V, Cipriani P, Bellisario B and Nascetti G. Parasitic infection by larval helminths in Antarctic fishes: pathological changes and impact on the host body condition index. Dis Aquat Org 2013; 105: 139-148.

376. Bahlool QZM, Skovgaard A, Kania PW and Buchmann K. Effects of excretory/secretory products from Anisakis simplex (Nematoda) on immune gene expression in rainbow trout (Oncorhynchus mykiss). Fish Shellfish Immunol 2013; 35: 734-739.

377. Audicana MT, Ansotegui IJ, de Corres LF and Kennedy MW. Anisakis simplex: dangerous-dead and alive? Trends Parasitol 2002; 18: 20-25.

378. Pascual S, Vega MA, Rocha FJ and Guerra A. First report of an endoparasitic epicaridean isopod infecting cephalopods. J Wildl Dis 2002; 38: 473-477.

379. Lafferty KD and Shaw JC. Comparing mechanisms of host manipulation across host and parasite taxa. $J$ Exp Biol 2013; 216: 56-66.

380. Poulin R. Parasite manipulation of host personality and behavioural syndromes. J Exp Biol 2013; 216: 18-26.

381. Cézilly F, Favrat A and Perrot-Minnot MJ. Multidimensionality in parasite-induced phenotypic alterations: ultimate versus proximate aspects. $J$ Exp Biol 2013; 216: 27-35.

382. Perrot-Minnot MJ and Cézilly F. Investigating candidate neuromodulatory systems underlying parasitic manipulation: concepts, limitations and prospects. J Exp Biol 2013; 216: 134-141.

383. Berk W, Teperman J, Walton KD, Hirata K, Sugimori $\mathrm{M}$ and Llinas RR. Oral administration of pharmacologically active substances to squid: a methodological d escription. Biol Bull 2009; 216: 1-6.

384. Hawkins P, Dennison N, Goodman G, Hetherington S, Llywelyn-Jones S, Ryder K and Smith AJ. Guidance on the severity classification of scientific procedures involving fish: report of a Working Group appointed by the Norwegian Consensus-Platform for the Replacement, Reduction and Refinement of animal experiments (Norecopa). Lab Anim 2011; 45: 219-224.

385. Morton DB and Griffiths PHM. Guidelines on the recognition of pain, distress and discomfort in experimental animals and a hypothesis for assessment. Vet Rec 1985; 116: 431-436.

386. Hawkins P. Recognizing and assessing pain, suffering and distress in laboratory animals: a survey of current practice in the UK with recommendations. Lab Anim 2002; 36: 378-395. 
387. Morton DB and Griffiths PHM. The recognition and measurement of pain and distress in laboratory animals. Lab Anim Sci 1985; 35: 540.

388. Amodio P and Fiorito G. Observational and other types of learning in Octopus. In: Menzel $\mathrm{R}$ and Benjamin $\mathrm{P}$ (eds) Invertebrate Learning and Memory. London: Academic Press, 2013, pp.293-302.

389. Wells MJ, Freeman NH and Ashburne M. Some experiments on chemotactile sense of octopuses. J Exp Biol 1965; 43: 553-563.

390. Darmaillacq AS, Dickel L, Chichery MP, Agin V and Chichery R. Rapid taste aversion learning in adult cuttlefish, Sepia officinalis. Anim Behav 2004; 68: 1291-1298.

391. Messenger JB. Learning in cuttlefish, Sepia. Anim Behav 1973; 21: 801-826.

392. Papini MR and Bitterman ME. Appetitive conditioning in Octopus cyanea. J Comp Psychol 1991; 105: 107-114.

393. Fiorito G, Biederman GB, Davey VA and Gherardi F. The role of stimulus preexposure in problem solving by Octopus vulgaris. Anim Cogn 1998; 1: 107-112.

394. Gutnick T, Byrne RA, Hochner B and Kuba M. Octopus vulgaris uses visual information to determine the location of its arm. Curr Biol 2011; 21: 460-462.

395. Agnisola C, Castaldo P and Fiorito G. Octopus vulgaris (Mollusca, Cephalopoda) as a model in behavioral pharmacology: A test of handling effects. Physiol Behav 1996; 59: 729-733.

396. Bardou I, Leprince J, Chichery R, Vaudry H and Agin V. Vasopressin/oxytocin-related peptides influence longterm memory of a passive avoidance task in the cuttlefish, Sepia officinalis. Neurobiol Learn Mem 2010; 93: 240-247.

397. Halm MP, Chichery MP and Chichery R. The role of cholinergic networks of the anterior basal and inferior frontal lobes in the predatory behaviour of Sepia officinalis. Comp Biochem Physiol Part A 2002; 132: 267-274.

398. Graindorge N, Jozet-Alves C, Chichery R, Dickel L and Bellanger C. Does kainic acid induce partial brain lesion in an invertebrate model: Sepia officinalis? Comparison with electrolytic lesion. Brain Res 2008; 1238: 44-52.

399. Johansen K and Martin AW. Circulation in cephalopod, Octopus dofleini. Comp Biochem Physiol 1962; 5: 165-176.

400. Smith LS. The role of venous peristalsis in the farm circulation of Octopus dofleini. Comp Biochem Physiol 1962; 7: 269-275.

401. Andrews PLR, Messenger JB and Tansey EM. Color changes in cephalopods after neurotransmitter injection into the cephalic aorta. Proc R Soc Lond B 1981; 213: 93-99.

402. Moreno H, Choi S, Yu E, Brusco J, Avila J, Moreira J, Sugimori M and Llinas RR. Blocking effects of human tau on squid giant synapse transmission and its prevention by T-817 MA. Frontiers in Synaptic Neuroscience $2011 ; 3$.

403. Grimaldi AM, Belcari P, Pagano E, Cacialli F and Locatello L. Immune responses of Octopus vulgaris (Mollusca: Cephalopoda) exposed to titanium dioxide nanoparticles. J Exp Mar Biol Ecol 2013; 447: 123-127.
404. Ponte G. Distribution and preliminary functional analysis of some modulators in the cephalopod mollusc Octopus vulgaris. $\mathrm{PhD}$ Thesis, Università della Calabria, Italy; Stazione Zoologica Anton Dohrn, Napoli, Italy, 2012.

405. Messenger JB. Neurotransmitters of cephalopods. Inv Neurosci 1996; 2: 95-114.

406. Mangold K and Bidder AM. Appareils respiratoie et circulatoire: respiration et circulation. In: Grassé PP and Mangold K (eds) Cephalopodes. Traite de Zoologie. Paris: Masson, 1989, pp.387-434.

407. Johansen K. Cardiac output in the large cephalopod Octopus dofleini. J Exp Biol 1965; 42: 475-480.

408. Lenfant $\mathrm{C}$ and Johansen K. Gas transport by hemocyanin-containing blood of the cephalopod Octopus dofleini. Amer J Physiol 1965; 209: 991-998.

409. Wolfensohn S and Lloyd M. Handbook of Laboratory Animal Management and Welfare. Oxford: Blackwell Science, 1998.

410. O'Dor RK and Wells MJ. Circulation time, blood reserves and extracellular space in a cephalopod. $J$ Exp Biol 1984; 113: 461-464.

411. Martin AW, Harrison FM, Huston MJ and Stewart DM. The blood volumes of some representative molluscs. J Exp Biol 1958; 35: 260-279.

412. Harms CA and Lewbart GA. Surgery in fish. Vet Clin North Am Exot Anim Pract 2000; 3: 759-774.

413. Murray MJ. Fish surgery. Seminars in Avian and Exotic Pet Medicine 2002; 11: 246-257.

414. Harms CA. Surgery in fish research: common procedures and postoperative care. Lab Anim (NY) 2005; 34: $28-34$.

415. Jennings $M$ and Berdoy M. Guiding Principles for Preparing for and Undertaking Aseptic Surgery. A Report by the LASA Education, Training and Ethics Section. Tamworth: LASA, 2010, p.16.

416. Hernndez A, Martró E, Matas L, Martín M and Ausina V. Assessment of in-vitro efficacy of $1 \%$ Virkon against bacteria, fungi, viruses and spores by means of AFNOR guidelines. J Hosp Inf 2000; 46: 203-209.

417. Pörtner HO, Webber DM, Boutilier RG and O'Dor RK. Acid-base regulation in exercising squid (Illex illecebrosus, Loligo pealei). Am J Physiol Regul Integr Comp Physiol 1991; 261: R239-R246.

418. Lewbart GA and Mosley C. Clinical anesthesia and analgesia in invertebrates. J Exot Pet Med 2012; 21: 59-70.

419. Ferguson GP, Messenger JB and Budelmann BU. Gravity and light influence the countershading reflexes of the cuttlefish Sepia officinalis. J Exp Biol 1994; 191: 247-256.

420. Shomrat T, Zarrella I, Fiorito G and Hochner B. The octopus vertical lobe modulates short-term learning rate and uses LTP to acquire long-term memory. Curr Biol 2008; 18: 337-342.

421. Chichery R and Chanelet J. Motor and behavioralresponses obtained by stimulation with chronic electrodes of optic lobe of Sepia officinalis. Brain Research 1976; 105: 525-532. 
422. Fiorito G and Chichery R. Lesions of the vertical lobe impair visual discrimination learning by observation in Octopus vulgaris. Neurosci Lett 1995; 192: 117-120.

423. Anderson ET, Davis AS, Law JM, Lewbart GA, Christian LS and Harms CA. Gross and histologic evaluation of 5 suture materials in the skin and subcutaneous tissue of the California sea hare (Aplysia californica). J Am Assoc Lab Anim Sci 2010; 49: 64-68.

424. Jackson GD, O'Dor RK and Andrade Y. First tests of hybrid acoustic/archival tags on squid and cuttlefish. Mar Freshwater Res 2005; 56: 425-430.

425. O'Dor RK and Webber DM. The constraints on cephalopods: why squid aren't fish. Can J Zool 1986; 64: 1591-1605.

426. Payne NL, Gillanders BM, Seymour RS, Webber DM, Snelling EP and Semmens JM. Accelerometry estimates field metabolic rate in giant Australian cuttlefish Sepia apama during breeding. J Anim Ecol 2011; 80: 422-430.

427. Yacob J, Lewis AC, Gosling A, St Hilaire DHJ, Tesar L, Mcrae M and Tublitz NJ. Principles underlying chromatophore addition during maturation in the European cuttlefish, Sepia officinalis. J Exp Biol 2011; 214: 3423-3432.

428. Sereni E and Young JZ. Nervous degeneration and regeneration in Cephalopods. Pubbl Staz Zool Napoli 1932; 12: 173-208.

429. Lange MM. On the regeneration and finer structure of the arms of the cephalopods. J Exp Zool 1920; 31: 1-57.

430. Feral JP. Wound-healing after arm amputation in Sepia officinalis (Cephalopoda, Sepioidea). J Invertebr Pathol J Invertebr Pathol 1988; 52: 380-388.

431. Fossati SM, Carella F, De Vico G, Benfenati F and Zullo L. Octopus arm regeneration: Role of acetylcholinesterase during morphological modification. J Exp Mar Biol Ecol 2013; 447: 93-99.

432. Florini $M$, Fiorito $G$, Hague $T$ and Andrews PLR. 'Monco': a natural model for studying arm usage and regeneration in Octopus vulgaris. J Shellfish Res 2011; 30: 1002.

433. Voight JR. Movement, injuries and growth of members of a natural population of the Pacific pygmy octopus, Octopus digueti. J Zool 1992; 228: 247-263.

434. Cummins SF, Boal JG, Buresch KC, Kuanpradit C, Sobhon P, Holm JB, Degnan BM, Nagle GT and Hanlon RT. Extreme aggression in male squid induced by a beta-MSP-like pheromone. Curr Biol 2011; 21: 322-327.

435. Hanlon RT, Ament SA and Gabr H. Behavioral aspects of sperm competition in cuttlefish, Sepia officinalis (Sepioidea: Cephalopoda). Mar Biol 1999; 134: 719-728.

436. Sinclair B, Briskey L, Aspden W and Pegg G. Genetic diversity of isolated populations of Nautilus pompilius (Mollusca, Cephalopoda) in the Great Barrier Reef and Coral Sea. Rev Fish Biol Fisher 2007; 17: 223-235.

437. Woodruff DS, Mulvey M, Saunders WB and Carpenter MP. Genetic variation in the cephalopod Nautilus belauensis. Proc Acad Nat Sci Philadelphia 1983; 135: 147-153.

438. Pagano E, Ponte G, Andrews PLR and Fiorito G. A Comparative analysis of different anaesthetics in octopus: towards true anesthesia? J Shellfish Res 2011; 30: 1016.

439. Gleadall IG. The effects of prospective anaesthetic substances on cephalopods: summary of original data and a brief review of studies over the last two decades. $J$ Exp Mar Biol Ecol 2013; 447: 23-30.

440. Goncalves RA, Aragao C, Frias PA and Sykes AV. The use of different anaesthetics as welfare promoters during short-term human manipulation of European cuttlefish (Sepia officinalis) juveniles. Aquaculture 2012; 370: 130-135.

441. Polese G, Winlow W and Di Cosmo A. Dose-dependent effects of the clinical anesthetic isoflurane on Octopus vulgaris: A contribution to cephalopod welfare. J Aquat Anim Health 2014; 26: 285-294.

442. Ellis TA, Narr BJ and Bacon DR. Developing a specialty: J.S. Lundy's three major contributions to anesthesiology. J Clin Anesth 2004; 16: 226-229.

443. Packard A. Uses of nicotine to follow denervation supersensitivity in unilaterally denervated octopus in vivo. J Physiol 1991; 438: 325.

444. Wood EM. Urethane as a carcinogen. The Progressive Fish-Culturist 1956; 18: 135-136.

445. Gunkel C and Lewbart GA. Anesthesia and analgesia of invertebrates. In: Fish RE, Brown MJ, Danneman PJaA and Karas AZ (eds) Anesthesia and Analgesia in Laboratory Animals (Second Edition). San Diego: Academic Press, 2008, pp.535-545.

446. Pantin CFA. Notes on Microscopical Technique for Zoologists. Cambridge: Cambridge University Press, 1946, p.91.

447. Messenger JB, Nixon M and Ryan KP. Magnesium chloride as an anesthetic for cephalopods. Comp Biochem Physiol C Comp Pharmacol 1985; 82: 203-205.

448. Gleadall IG. Comparison of anaesthetics for octopuses. Bull Mar Sci 1991; 49: 663.

449. Seol DW, Lee J, Im SY and Park IS. Clove oil as an anaesthetic for common octopus (Octopus minor, Sasaki). Aquac Res 2007; 38: 45-49.

450. Barord GJ and Christie BL. Benzocaine in the euthanasia of the giant Pacific octopus, Enteroctopus dofleini (Wulker, 1919). Drum and Croaker 2007; 38: 7-12.

451. O'Dor RK, Pörtner HO and Shadwick RE. Squid as elite athletes: Locomotory, respiratory and circulatory integration. In: Gilbert DL, Adelman WJ, Arnold JM, editors. Squid as Experimental Animals. New York: 1990, pp. 481-503.

452. Zahl IH, Samuelsen O and Kiessling A. Anaesthesia of farmed fish: implications for welfare. Fish Physiol Biochem 2012; 38: 201-218.

453. Melzner F, Bock C and Pörtner HO. Thermal sensitivity of the venous return system in the cephalopod Sepia officinalis. Comp Biochem Phys A 2007; 146: S165.

454. Melzner F, Bock C and Pörtner HO. Coordination between ventilatory pressure oscillations and venous return in the cephalopod Sepia officinalis under control conditions, spontaneous exercise and recovery. J Comp Physiol B 2007; 177: 1-17.

455. Melzner F, Bock C and Pörtner HO. Critical temperatures in the cephalopod Sepia officinalis investigated 
using in vivo 31P NMR spectroscopy. J Exp Biol 2006; 209: 891-906.

456. Andrews PLR, Messenger JB and Tansey EM. The chromatic and motor effects of neurotransmitter injection in intact and brain-lesioned Octopus. J Mar Biol Ass U K 1983; 63: 355-370.

457. Flachsenberger W and Kerr DIB. Lack of effect of tetrodotoxin and of an extract from the posterior salivary-gland of the blue-ringed octopus following injection into the octopus and following application to its brachial nerve. Toxicon 1985; 23: 997-999.

458. Demers G, Griffin G, De Vroey G, Haywood JR, Zurlo $\mathbf{J}$ and Bedard M. Harmonization of animal care and use guidance. Science 2006; 312: 700-701.

459. Canadian Council on Animal Care. CCAC Guidelines on: euthanasia of animal used in science. Ottawa: Canadian Council on Animal Care, 2010, p.36.

460. Hawkins P, Playle L, Golledge H, Leach M, Banzett R, Coenen A, Cooper J, Danneman P, Flecknell P and Kirkden R. Newcastle consensus meeting on carbon dioxide euthanasia of laboratory animals. London: NC3Rs, 2006, p.17.

461. Neill D. The effect of the Crustastun on nerve activity in crabs and lobsters. Glasgow, UK: University of Scotland and Scientific Report to Studham Technologies Ltd. 2010, p.21.

462. Fiedler A and Schipp R. The role of the branchial heart complex in circulation of coleoid cephalopods. Experientia 1987; 43: 544-553.

463. Smith PJS. The role of venous pressure in regulation of output from the heart of the octopus, Eledone cirrhosa (Lam.). J Exp Biol 1981; 93: 243-255.

464. Ballering RB, Jalving MA, VenTresca DA, Hallacher LE, Tomlinson JT and Wobber DR. Octopus evenomation through a plastic bag via a salivary proboscis. Toxicon 1972; 10: 245-248.

465. Cariello L and Zanetti L. Alpha-Cephalotoxin and Beta-Cephalotoxin: Two paralyzing proteins from posterior salivary glands of Octopus vulgaris. Comp Biochem Physiol, Part C: Pharmacol Toxicol Endocrinol 1977; 57: 169-173.

466. Ruder T, Sunagar K, Undheim EA, Ali SA, Wai TC, Low DH, Jackson TN, King GF, Antunes A and Fry BG. Molecular phylogeny and evolution of the proteins encoded by Coleoid (cuttlefish, octopus, and squid) posterior venom glands. J Mol Evol 2013; 76: 192-204.

467. Walker DG. Survival after severe envenomation by the blue-ringed octopus (Hapalochlaena maculosa). Medical Journal of Australia 1983; 2: 663-665.

468. Cavazzoni E, Lister B, Sargent P and Schibler A. Blueringed octopus (Hapalochlaena sp.) envenomation of a 4-year-old boy: A case report. Clin Toxicol 2008; 46: $760-761$.

469. Sheumack DD, Howden MEH, Spence I and Quinn RJ. Maculotoxin - Neurotoxin from venom glands of octopus Hapalochlaena maculosa identified as Tetrodotoxin. Science 1978; 199: 188-189.

470. Williams BL, Stark MR and Caldwell RL. Microdistribution of tetrodotoxin in two species of blue-ringed octopuses (Hapalochlaena lunulata and Hapalochlaena fasciata) detected by fluorescent immunolabeling. Toxicon 2012; 60: 1307-1313.

471. Williams BL, Lovenburg V, Huffard CL and Caldwell RL. Chemical defense in pelagic octopus paralarvae: Tetrodotoxin alone does not protect individual paralarvae of the greater blue-ringed octopus (Hapalochlaena lunulata) from common reef predators. Chemoecology 2011; 21: 131-141.

472. Hwang DF, Arakawa O, Saito T, Noguchi T, Simidu U, Tsukamoto K, Shida $\mathrm{Y}$ and Hashimoto K. Tetrodotoxin-producing bacteria from the blue-ringed octopus Octopus maculosus. Mar Biol 1989; 100: 327-332.

473. Ottuso P. Aquatic dermatology: encounters with the denizens of the deep (and not so deep) a review. Part I: the invertebrates. Int $J$ Dermatol 2013; 52: 136-152.

474. Anderson RC, Hughes PD, Mather JA and Steele CW. Determination of the diet of Octopus rubescens Berry, 1953 (Cephalopoda: Octopodidae), through examination of its beer bottle dens in Puget Sound. Malacologia 1999; 41: 455-460.

475. Key LN, Boyle PR and Jaspars M. Novel activities of saliva from the octopus Eledone cirrhosa (Mollusca; Cephalopoda). Toxicon 2002; 40: 677-683.

476. Misago N, Inoue T and Narisawa Y. Delayed reaction after an octopus bite showing a giant cell-rich granulomatous dermatitis/panniculitis. J Cutan Pathol 2008; 35: 1068-1072.

477. Derby CD. Escape by inking and secreting: marine molluscs avoid predators through a rich array of chemicals and mechanisms. Biol Bull 2007; 213: 274-289.

478. Derby $C$. The role of chemicals in interactions between inking molluscs and their predators. Integr Comp Biol 2013; 53: E51.

479. Yang K, LeJeune J, Alsdorf D, Lu B, Shum CK and Liang S. Global distribution of outbreaks of water-associated infectious diseases. PLoS Negl Trop Dis 2012; 6: e1483.

480. Aigner BA, Ollert M, Seifert F, Ring J and Plotz SG. Pseudomonas oryzihabitans cutaneous ulceration from Octopus vulgaris bite A case report and review of the literature. Archives of Dermatology 2011; 147: 963-966.

481. Campanelli A, Sanchez-Politta S and Saurat JH. [Cutaneous ulceration after an octopus bite: infection due to Vibrio alginolyticus, an emerging pathogen] original title in French. Ann Dermatol Venereol 2008; 135: 225-227.

482. Aigner BA, Ollert M, Seifert F, Plotz SG and Ring J. Cutaneous ulceration and wound healing deficit after octopus-bite. Journal der Deutschen Dermatologischen Gesellschaft 2011; 9: 158-159.

483. Shen HW, Cao MJ, Cai QF, Ruan MM, Mao HY, Su WJ and Liu GM. Purification, cloning, and immunological characterization of arginine kinase, a novel allergen of Octopus fangsiao. J Agric Food Chem 2012; 60: 2190-2199. 
484. Hawkins P, Morton DB, Burman O, Dennison $\mathrm{N}$, Honess P, Jennings M, Lane S, Middleton V, Roughan $\mathrm{JV}$, Wells $\mathrm{S}$ and Westwood K. A guide to defining and implementing protocols for the welfare assessment of laboratory animals. Eleventh report of the BVAAWF/ FRAME/RSPCA/UFAW Joint Working Group on Refinement. Lab Anim 2011; 45: 1-13.

485. Hooijmans CR, Leenaars M and Ritskes-Hoitinga M. A Gold Standard Publication Checklist to improve the quality of animal studies, to fully integrate the Three Rs, and to make systematic reviews more feasible. ATLA: Altern Lab Anim 2010; 38: 167-182.

486. Hooijmans C, de Vries R, Leenaars M and RitskesHoitinga M. The Gold Standard Publication Checklist (GSPC) for improved design, reporting and scientific quality of animal studies GSPC versus ARRIVE guidelines. Lab Anim 2011; 45: 61.

487. Ponte $\mathrm{G}$ and Fiorito G. A special issue on Cephalopod Biology: contributions of a community to the advancements of the biology of cephalopods. J Exp Mar Biol Ecol 2013; 447: 1-3.

488. Nevalainen T, Blom HJM, Guaitani A, Hardy P, Howard BR and Vergara P. FELASA recommendations for the accreditation of laboratory animal science education and training: Report of the Federation of European Laboratory Animal Science Associations Working Group on Accreditation of Laboratory Animal Science Education and Training. Lab Anim 2002; 36: 373-377.

489. FELASA. Recommendations for the Accreditation of Education and Training courses in Laboratory Animal Science. 2014, p. 14.

490. Lindl T, Gross U, Ruhdel I, von Aulock S and Volker M. Guidance on determining indispensability and balancing potential benefits of animal experiments with costs to the animals with specific consideration of EU Directive 2010/63/EU. Altex-Alternatives to Animal Experimentation 2012; 29: 219-228.

491. Bullock TH. Mollusca: Cephalopoda. In: Bullock TH and Horridge GA (eds) Structure and Function in the Nervous Systems of Invertebrates. San Francisco and London: W.H. Freeman and Company, 1965, pp.1433-1515.

492. Budelmann BU and Young JZ. Central pathways of the nerves of the arms and mantle of Octopus. Philos Trans $R$ Soc Lond B 1985; 310: 109-122.

493. Budelmann BU and Young JZ. Brain pathways of the brachial nerves of Sepia and Loligo. Philos Trans $R$ Soc Lond B 1987; 315: 345-352.

494. Edelman DB and Seth AK. Animal consciousness: a synthetic approach. Trends Neurosci 2009; 32: 476-484.

495. Edelman DB. How octopuses see the world and other roads less traveled: necessity versus sufficiency and evolutionary convergence in the study of animal consciousness. J Shellfish Res 2011; 30: 1001.

496. Zhu W and Stefano GB. Comparative aspects of endogenous morphine synthesis and signaling in animals. Ann Rev NY Acad Sci-Trends in Comparative Endocrinology and Neurobiology 2009; 1163: 330-339.
497. Sha AL, Sun HS and Wang Y. Immunohistochemical localization of met-enkephalin in mantles and feet of octopus Octopus ocellatus Grey. Fish Sci 2007; 26: 622-624.

498. Sha AL, Sun HS and Wang YY. Immunohistochemical study of leucine-enkephalin and delta opioid receptor in mantles and feet of the octopus Octopus ocellatus Gray. Int J Pept Res Ther 2012; 18: 71-76.

499. De Lisa E, Paolucci M and Di Cosmo A. Conservative nature of oestradiol signalling pathways in the brain lobes of Octopus vulgaris involved in reproduction, learning and motor coordination. J Neuroendocrinol 2012; 24: 275-284.

500. Sha A, Sun $H$ and Wang Y. Immunohistochemical observations of methionine-enkephalin and delta opioid receptor in the digestive system of Octopus ocellatus. Tissue Cell 2013; 45: 83-87.

501. Stefano GB, Hall B, Makman MH and Dvorkin B. Opioid inhibition of dopamine release from nervoustissue of Mytilus edulis and Octopus bimaculatus. Science 1981; 213: 928-930.

502. Frazier DT, Ohta M and Narahash T. Nature of morphine receptor present in squid axon. Proc Soc Exp Biol Med 1973; 142: 1209-1214.

503. Robertson JD, Bonaventura J and Kohm A. Nitric oxide synthase inhibition blocks octopus touch learning without producing sensory or motor dysfunction. Proc $R$ Soc Lond B 1995; 261: 167-172.

504. Purdy JE, Dixon D, Estrada A, Peters A, Riedlinger E and Suarez R. Prawn-in-a-tube procedure: Habituation or associative learning in cuttlefish? J Gen Psychol 2006; 133: 131-152.

505. Dickel L, Boal JG and Budelmann BU. The effect of early experience on learning and memory in cuttlefish. Dev Psychobiol 2000; 36: 101-110.

506. Agin W, Chichery R, Dickel L and Chichery MP. The 'prawn-in-the-tube' procedure in the cuttlefish: Habituation or passive avoidance learning? Learn Memory 2006; 13: 97-101.

507. Cartron L, Darmaillacq A-S and Dickel L. The 'prawnin-the-tube' procedure: What do cuttlefish learn and memorize? Behav Brain Res 2013; 240: 29-32.

508. Lee PG, Forsythe JW, DiMarco FP, Derusha RH and Hanlon RT. Initial palatability and growth trials on pelleted diets for cephalopods. Bull Mar Sci 1991; 49: 362-372.

509. Castro BG, DiMarco PF, Derusha RH and Lee PG. The effects of surimi and pelleted diets on the laboratory survival, growth, and feeding rate of the cuttlefish Sepia officinalis L. J Exp Mar Biol Ecol 1993; 170: 241-252.

510. Castro BG and Lee PG. The effects of semi-purified diets on growth and condition of Sepia officinalis L. (Mollusca: Cephalopoda). Comp Biochem Physiol Part A: Physiology 1994; 109: 1007-1016.

511. Domingues PM. Development of alternative diets for the mass culture of the European cuttlefish Sepia officinalis. Thesis, University of Algarve, Faro, Portugal, 1999. 
512. Domingues P, Ferreira A, Marquez L, Andrade JP, Lopez N and Rosas C. Growth, absorption and assimilation efficiency by mature cuttlefish (Sepia officinalis) fed with alternative and artificial diets. Aquacult Int 2008; 16: 215-229.

513. Aguila J, Cuzon G, Pascual C, Domingues PM, Gaxiola G, Sanchez A, Maldonado T and Rosas C. The effects of fish hydrolysate (CPSP) level on Octopus maya (Voss and Solis) diet: Digestive enzyme activity, blood metabolites, and energy balance. Aquaculture 2007; 273: 641-655.

514. Domingues PM, Lopez N, Munoz JA, Maldonado T, Gaxiola $G$ and Rosas C. Effects of a dry pelleted diet on growth and survival of the Yucatan octopus, Octopus maya. Aquac Nutr 2007; 13: 273-280.

515. Rosas C, Cuzon G, Pascual C, Gaxiola G, Chay D, Lopez N, Maldonado T and Domingues PM. Energy balance of Octopus maya fed crab or an artificial diet. Mar Biol 2007; 152: 371-381.

516. Garcia S, Domingues P, Navarro JC, Hachero I, Garrido D and Rosas C. Growth, partial energy balance, mantle and digestive gland lipid composition of Octopus vulgaris (Cuvier, 1797) fed with two artificial diets. Aquac Nutr 2011; 17: E174-E187.

517. Valverde JC, Hernandez MD, Aguado-Gimenez F and Garcia BG. Growth, feed efficiency and condition of common octopus (Octopus vulgaris) fed on two formulated moist diets. Aquaculture 2008; 275: 266-273.

518. Quintana D, Domingues P and Garcì S. Effect of two artificial wet diets agglutinated with gelatin on feed and growth performance of common octopus (Octopus vulgaris) sub-adults. Aquaculture 2008; 280: 161-164.

519. Garcia-Garrido S, Hachero-Cruzado I, Domingues P, Lopez $\mathrm{N}$ and Rosas C. Effects of fish hydrolysate (CPSP (R)) on growth and digestive gland lipid composition of Octopus vulgaris (Cuvier, 1797) juveniles. Aquac Nutr 2011; 17: E825-E839.

520. Morillo-Velarde PS, Valverde JC, Hernandez MD, Aguado-Gimenez F and Garcia BG. Growth and digestibility of formulated diets based on dry and freeze-dried ingredients in the common octopus (Octopus vulgaris). Aquaculture 2012; 368: 139-144.

521. Cagnetta P and Sublimi A. Productive performance of the common octopus (Octopus vulgaris $\mathrm{C}$.) when fed on a monodiet. Recent Advances in Mediterranean Aquaculture Finfish Species Diversification. Zaragoza; Paris: CIHEAM, 2000, pp. 331-336.

522. Estefanell J, Socorro J, Tuya F, Izquierdo M and Roo J. Growth, protein retention and biochemical composition in Octopus vulgaris fed on different diets based on crustaceans and aquaculture by-products. Aquaculture 2011; 322: 91-98.

523. Estefanell J, Roo J, Guirao R, Izquierdo M and Socorro J. Benthic cages versus floating cages in Octopus vulgaris: Biological performance and biochemical composition feeding on Boops boops discarded from fish farms. Aquac Eng 2012; 49: 46-52.

524. Estefanell J, Roo J, Guirao R, Afonso JM, FernandezPalacios H, Izquierdo M and Socorro J. Efficient utilization of dietary lipids in Octopus vulgaris (Cuvier 1797) fed fresh and agglutinated moist diets based on aquaculture by-products and low price trash species. Aquac Res 2012; 44: 93-105.

525. Petza D, Katsanevakis $\mathrm{S}$ and Verriopoulos G. Experimental evaluation of the energy balance in Octopus vulgaris, fed ad libitum on a high-lipid diet. Mar Biol 2006; 148: 827-832.

526. Estefanell $\mathrm{J}$, Socorro $\mathrm{J}$, Izquierdo $\mathrm{M}$ and Roo $\mathrm{J}$. Growth, food intake, protein retention and fatty acid profile in Octopus vulgaris (Cuvier, 1797) fed agglutinated moist diets containing fresh and dry raw materials based on aquaculture by-products. Aquac Res 2013; 45: 54-67.

527. Cagnetta P. Preliminary observations on the productive responses of the common octopus (Octopus vulgaris C.) reared free or in individual nets. Recent Advances in Mediterranean Aquaculture Finfish Species Diversification. Zaragoza; Paris: CIHEAM, 2000, pp. 323-329.

528. Kristinsson HG and Rasco BA. Fish protein hydrolysates: Production, biochemical, and functional properties. Critical Reviews in Food Science and Nutrition 2000; 40: 43-81.

529. Leibovitz L, Meyers TR, Elston R and Chanley P. Necrotic exfoliative dermatitis of captive squid (Loligo pealei). J Invert Pathol 1977; 30: 369-376.

530. Gore SR, Harms CA, Kukanich B, Forsythe J, Lewbart GA and Papich MG. Enrofloxacin pharmacokinetics in the European cuttlefish, Sepia officinalis, after a single i.v. injection and bath administration. $J$ Vet Pharmacol Ther 2005; 28: 433-439.

531. Reimschuessel R, Stoskopf MK and Bennett R. Myocarditis in the common cuttlefish (Sepia officinalis). J Comp Pathol 1990; 102: 291-297.

532. Rosa R, Pimentel MS, Boavida-Portugal J, Teixeira T, Trübenbach K and Diniz M. Ocean warming enhances malformations, premature hatching, metabolic suppression and oxidative stress in the early life stages of a keystone squid. PLoS ONE 2012; 7: e38282.

533. Zullo L, Sumbre G, Agnisola C, Flash T and Hochner B. Nonsomatotopic organization of the higher motor centers in octopus. Curr Biol 2009; 19: 1632-1636.

534. Lamarre SG, Ditlecadet D, McKenzie DJ, Bonnaud L and Driedzic WR. Mechanisms of protein degradation in mantle muscle and proposed gill remodeling in starved Sepia officinalis. Am J Physiol Regul Integr Comp Physiol 2012; 303: R427-R437.

535. Grigoriou P and Richardson CA. Effect of body mass, temperature and food deprivation on oxygen consumption rate of common cuttlefish Sepia officinalis. Mar Biol 2009; 156: 2473-2481.

536. Fiorito G, Agnisola C, d'Addio M, Valanzano A and Calamandrei G. Scopolamine impairs memory recall in Octopus vulgaris. Neurosci Lett 1998; 253: 87-90.

537. Wells MJ and Wells J. Fluid uptake and the maintenance of blood volume in octopus. 1993; 175: 211-218.

538. Robertson JD, Bonaventura $\mathbf{J}$ and Kohm AP. Nitric oxide is required for tactile learning in Octopus vulgaris. Proc R Soc Lond B 1994; 256: 269-273. 
539. Robertson JD, Bonaventura J, Kohm A and Hiscat M. Nitric oxide is necessary for visual learning in Octopus vulgaris. Proc $R$ Soc Lond B 1996; 263: 1739-1743.

540. Tansey EM. A histochemical study of the cephalopod brain. PhD Thesis, University of Sheffield, UK, 1978.

541. Gaston MR and Tublitz NJ. Peripheral innervation patterns and central distribution of fin chromatophore motoneurons in the cuttlefish Sepia officinalis. $J$ Exp Biol 2004; 207: 3089-3098.

542. Juorio AV. Catecholamines and 5-hydroxytryptamine in nervous tissue of cephalopods. J Physiol 1971; 216: 213-226.

543. Beuerlein K, Lohr S, Westermann B, Ruth P and Schipp $\mathrm{R}$. Components of the cellular defense and detoxification system of the common cuttlefish Sepia officinalis (Mollusca, Cephalopoda). Tissue Cell 2002; 34: 390-396.
544. Beuerlein K, Ruth P, Westermann B, Lohr S and Schipp R. Hemocyanin and the branchial heart complex of Sepia officinalis: are the hemocytes involved in hemocyanin metabolism of coleoid cephalopods? Cell Tissue Res 2002; 310: 373-381.

545. Wells MJ and Wells J. Ventilatory currents in the mantle of cephalopods. J Exp Biol 1982; 99: 315-330.

546. Ikeda Y, Sugimoto C, Yonamine H and Oshima Y. Method of ethanol anaesthesia and individual marking for oval squid (Sepioteuthis lessoniana Ferussac, 1831 in Lesson 1830-1831). Aquac Res 2009; 41: 157-160.

547. Kier WM, Smith KK and Miyan JA. Electromyography of the fin musculature of the cuttlefish Sepia officinalis. $J$ Exp Biol 1989; 143: 17-31.

548. Gutfreund Y, Matzner H, Flash $\mathrm{T}$ and Hochner B. Patterns of motor activity in the isolated nerve cord of the octopus arm. Biol Bull 2006; 211: 212-222. 
Appendix 1.

Summary of key information required in a project application under the Directive (see for further detailed information Smith et al., 2013) ${ }^{1}$

\section{Information required in applications for project authorisation}

Directive 2010/63/EU Annex VI and Article 38(2)e

1. Relevance and justification of the following:

(a) use of animals including their origin, estimated numbers, species and life stages;

(b) procedures.

2. Application of methods to replace, reduce and refine the use of animals in procedures.

3. The planned use of anaesthesia, analgesia and other pain relieving methods.

4. Reduction, avoidance and alleviation of any form of animal suffering, from birth to death where appropriate.

5. Use of humane end-points.

6. Experimental or observational strategy and statistical design to minimise animal numbers, pain, suffering, distress and environmental impact where appropriate.

7. Re-use of animals and its accumulative effect on the animals.

8. The proposed severity classification of procedures.

9. Avoidance of unjustified duplication of procedures where appropriate.

10. Housing, husbandry and care conditions for the animals.

11. Methods of killing.

12. Competence of persons involved in the project

\section{Purposes of procedures permitted}

Directive 2010/63/EU Article 5

Procedures may be carried out for the following purposes only:

(a) basic research;

(b) translational or applied research with any of the following aims:

(i) the avoidance, prevention, diagnosis or treatment of disease, ill-health or other abnormality or their effects in human beings, animals or plants.

(ii) the assessment, detection, regulation or

${ }^{1}$ For full reference to the cited document see Reference List (\#3) at the end of the paper. modification of physiological conditions in human beings, animals or plants.

(iii) the welfare of animals and the improvement of the production conditions for animals reared for agricultural purposes

(c) for any of the aims in (b), in the development, manufacture or testing of the quality, effectiveness and safety of drugs, food- and feed-stuffs and other substances or products;

(d) protection of the natural environment in the interests of the health or welfare of human beings or animals;

(e) research aimed at preservation of the species;

(f) higher education, or training for the acquisition, maintenance or improvement of vocational skills;

(g) forensic inquiries.

Questions that should be addressed in relation to the Three Rs in writing a project application, and in the project review and approval process

\section{Replacement}

(i) What on-going efforts will you make to identify 'scientifically satisfactory' alternative methods that could replace the use of some or all animals? (Article 4§ 1)

(ii) Could you avoid the use of animals by asking different type of question or making better use of existing data or literature to address the scientific objectives?

(iii) Could in vitro studies or in silico (computer)-modelling be used to replace some or all of the animals?

\section{Reduction}

(iv) How will you ensure that the number of animals used in the project, and in individual studies within the project, is 'reduced to a minimum without compromising the scientific objectives'? (Article 4\$2)

(v) Could any further reductions be made, e.g. by taking expert statistical advice to help optimise experimental and statistical design?

\section{Refinement}

(vi) How have you refined the 'breeding, accommodation and care of the animals' and the 'methods used in procedures', so as to 'reduce to the 
minimum any possible pain, suffering, distress or lasting harm to the animals' throughout their lives? (Article 4§3)

(vii) Have you considered and implemented all the possibilities for refinement described elsewhere in these guidelines? (see Smith et al. 2013 for examples of refinement in the context of specific procedures).

(viii) How will you ensure that all relevant personnel working on the project are adequately educated and trained, and are supervised until they have demonstrated their competence in the procedures? (see section 10) 
용

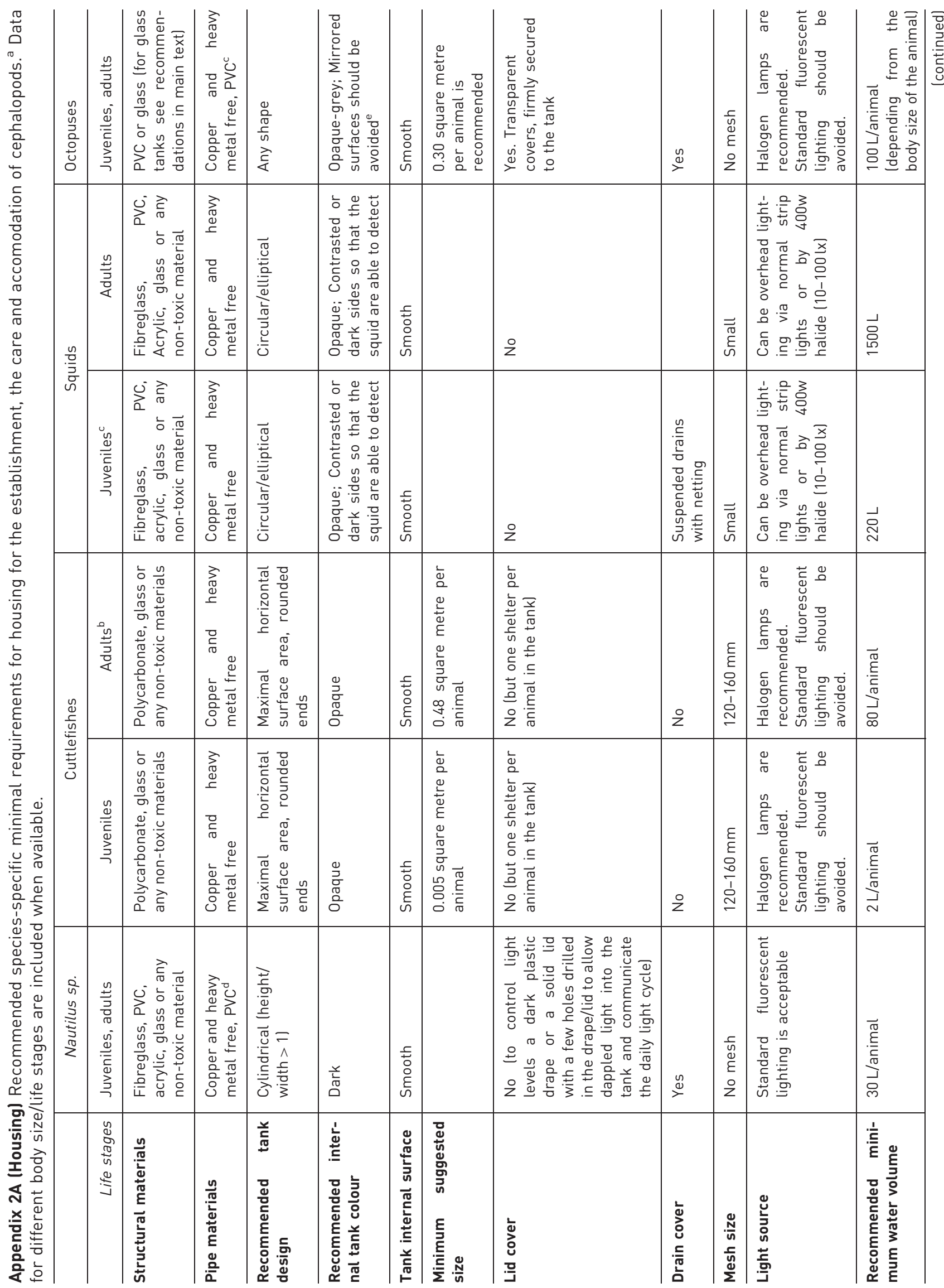




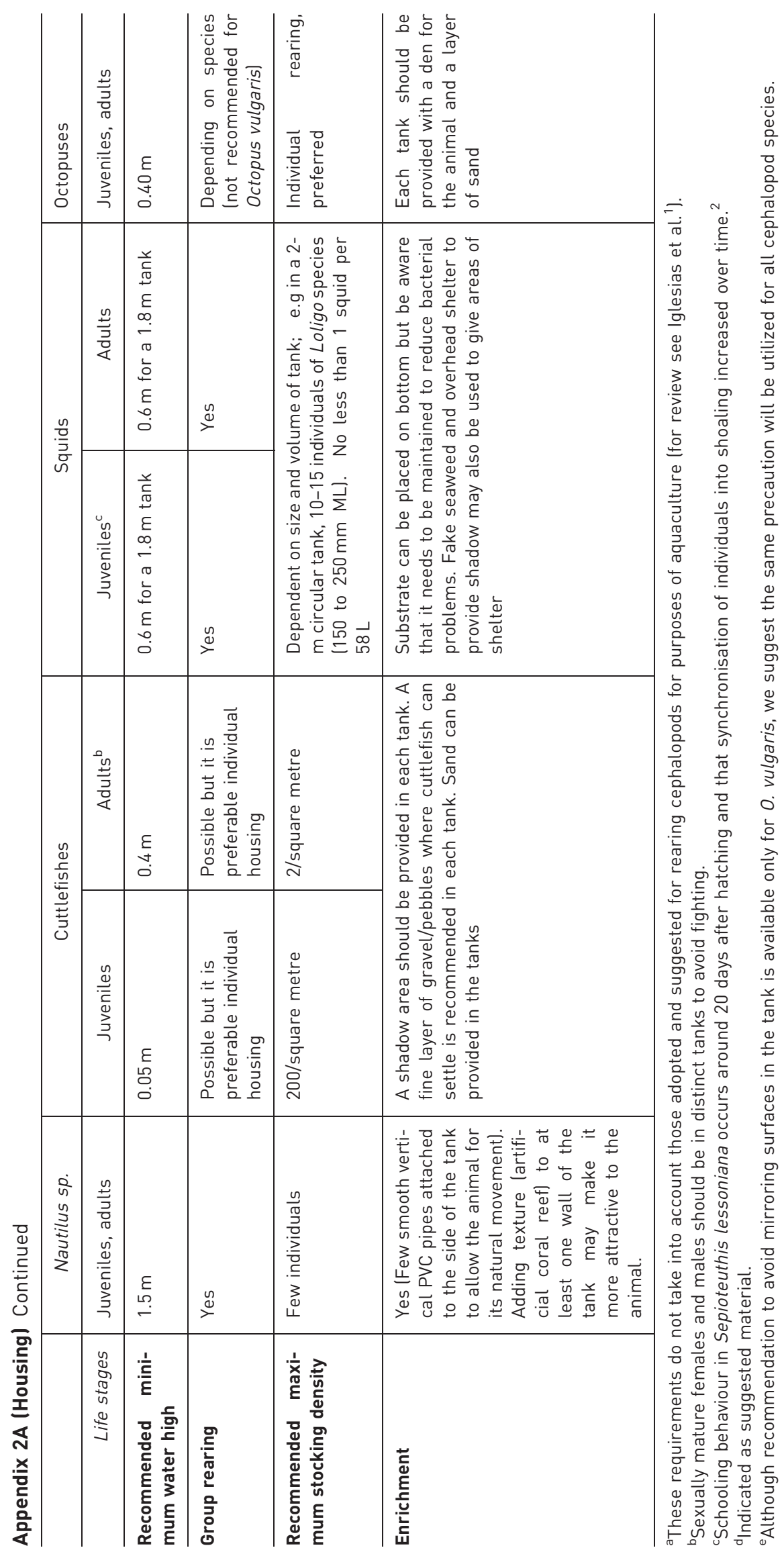




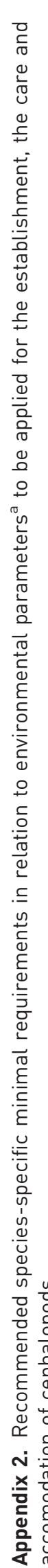

\begin{tabular}{|c|c|c|c|c|c|c|c|c|c|c|c|c|}
\hline 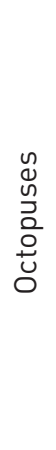 & 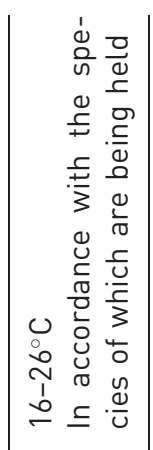 & $\begin{array}{c}+\frac{0}{2} \\
2 \\
\tilde{2} \\
1 \\
\tilde{N} \\
\tilde{1}\end{array}$ & 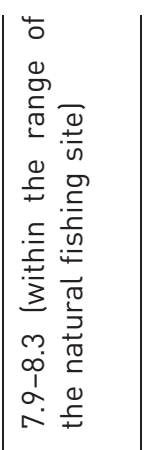 & $\left|\begin{array}{c}\perp \\
\delta \\
\varepsilon \\
\stackrel{0}{0} \\
\dot{0} \\
v\end{array}\right|$ & 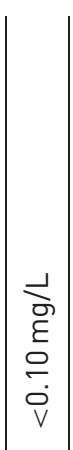 & $\begin{array}{c}\vec{J} \\
\text { Dे } \\
\text { D } \\
\text { V }\end{array}$ & 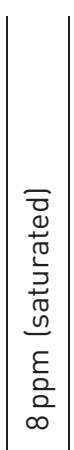 & 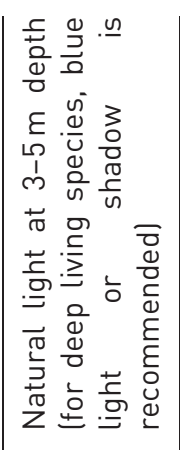 & 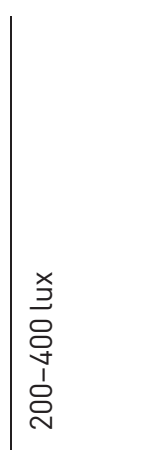 & 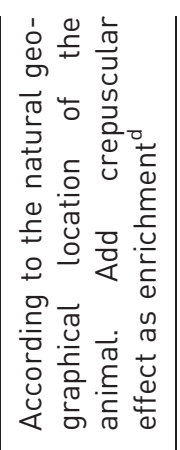 & 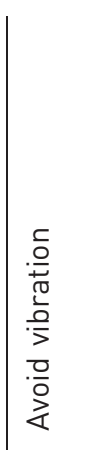 & 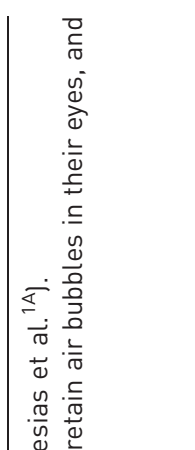 \\
\hline $\begin{array}{l}\frac{n}{0} \\
\frac{0}{3} \\
\bar{n}\end{array}$ & 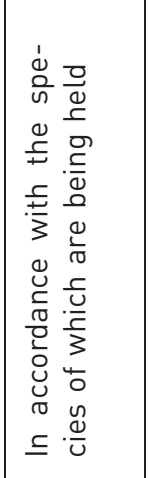 & 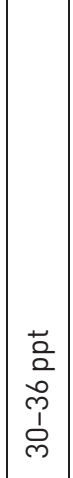 & 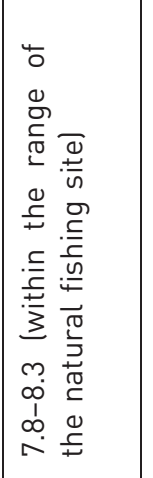 & $\begin{array}{c}\sigma \\
\bar{\sigma} \\
\varepsilon \\
\bar{\sigma} \\
\dot{0} \\
v\end{array}$ & 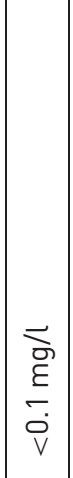 & 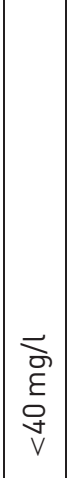 & 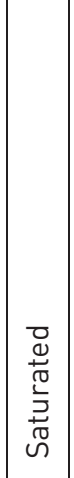 & 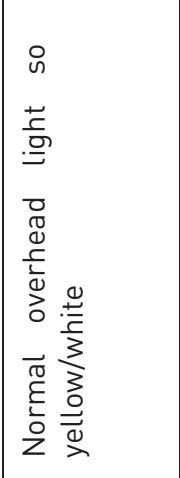 & 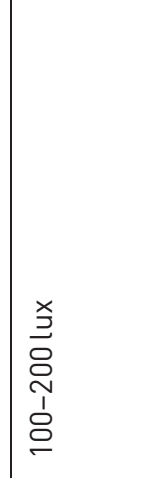 & 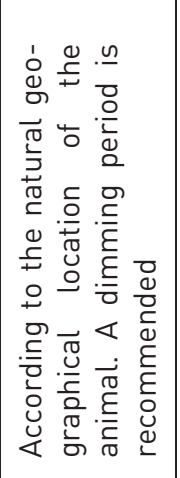 & 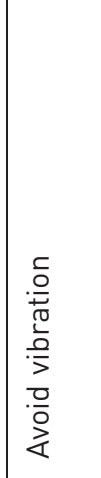 & 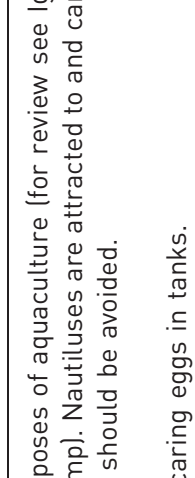 \\
\hline 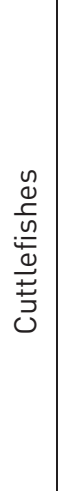 & 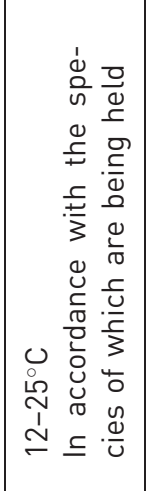 & 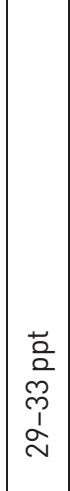 & 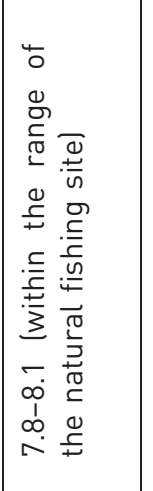 & 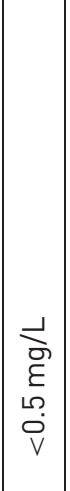 & $\left|\begin{array}{c}\vec{J} \\
\text { Dे } \\
\dot{E} \\
\dot{v} \\
v\end{array}\right|$ & $\begin{array}{c}\vec{J} \\
\text { के } \\
\varepsilon \\
D \\
\text { v }\end{array}$ & 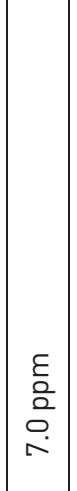 & 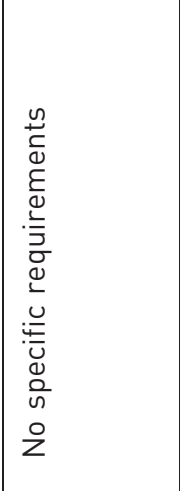 & $\begin{array}{l}\stackrel{\times}{\Xi} \\
\text { ○् } \\
\stackrel{\text { }}{V}\end{array}$ & 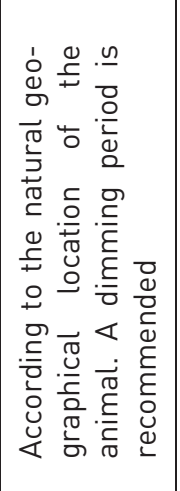 & 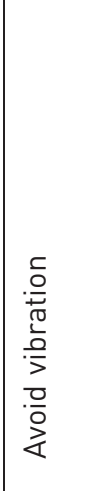 & 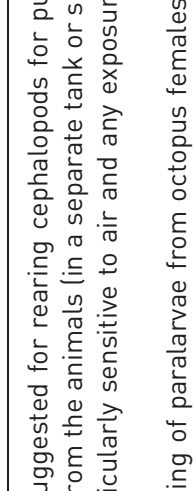 \\
\hline 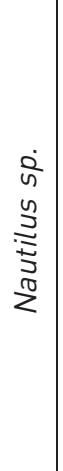 & 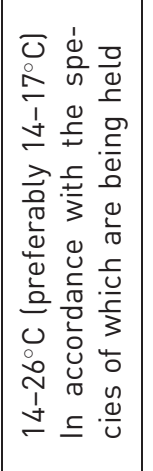 & 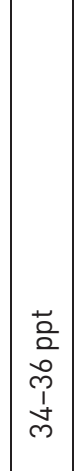 & 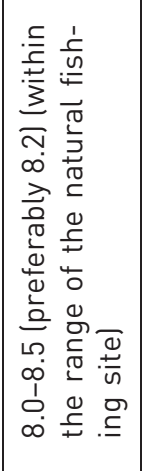 & 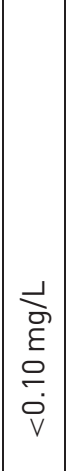 & 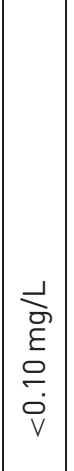 & $\begin{array}{c}\perp \\
\text { Dे } \\
\varepsilon \\
D \\
\text { V }\end{array}$ & 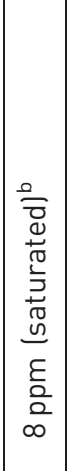 & 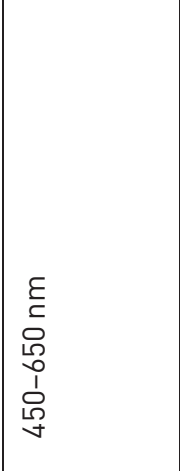 & & 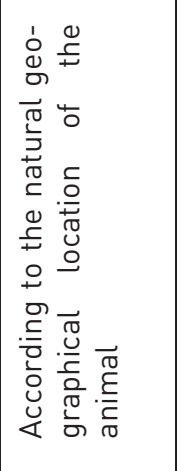 & 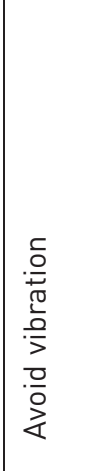 & 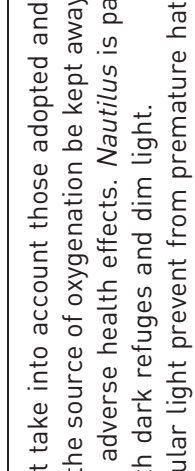 \\
\hline & 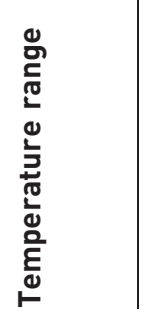 & 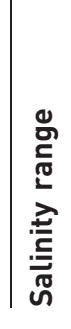 & 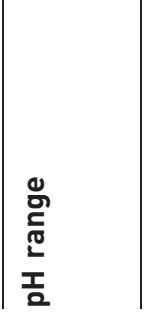 & 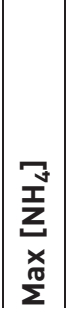 & $\left|\begin{array}{l}\bar{N} \\
0 \\
z \\
x \\
\frac{\pi}{\Sigma}\end{array}\right|$ & 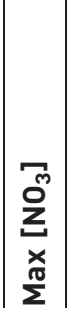 & 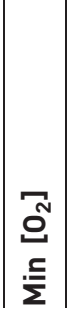 & 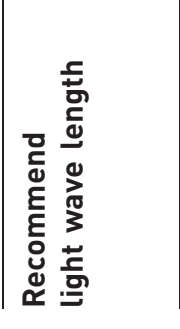 & 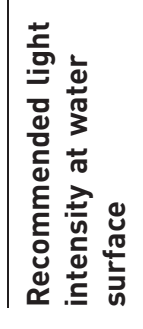 & $\begin{array}{l}\text { 은 } \\
\frac{0}{0} \\
\frac{0}{2} \\
\frac{0}{0} \\
\frac{c}{0}\end{array}$ & 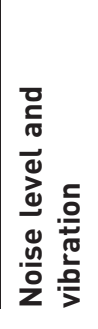 & 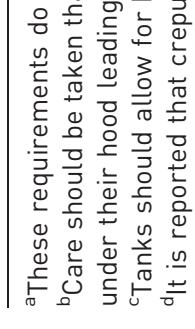 \\
\hline
\end{tabular}




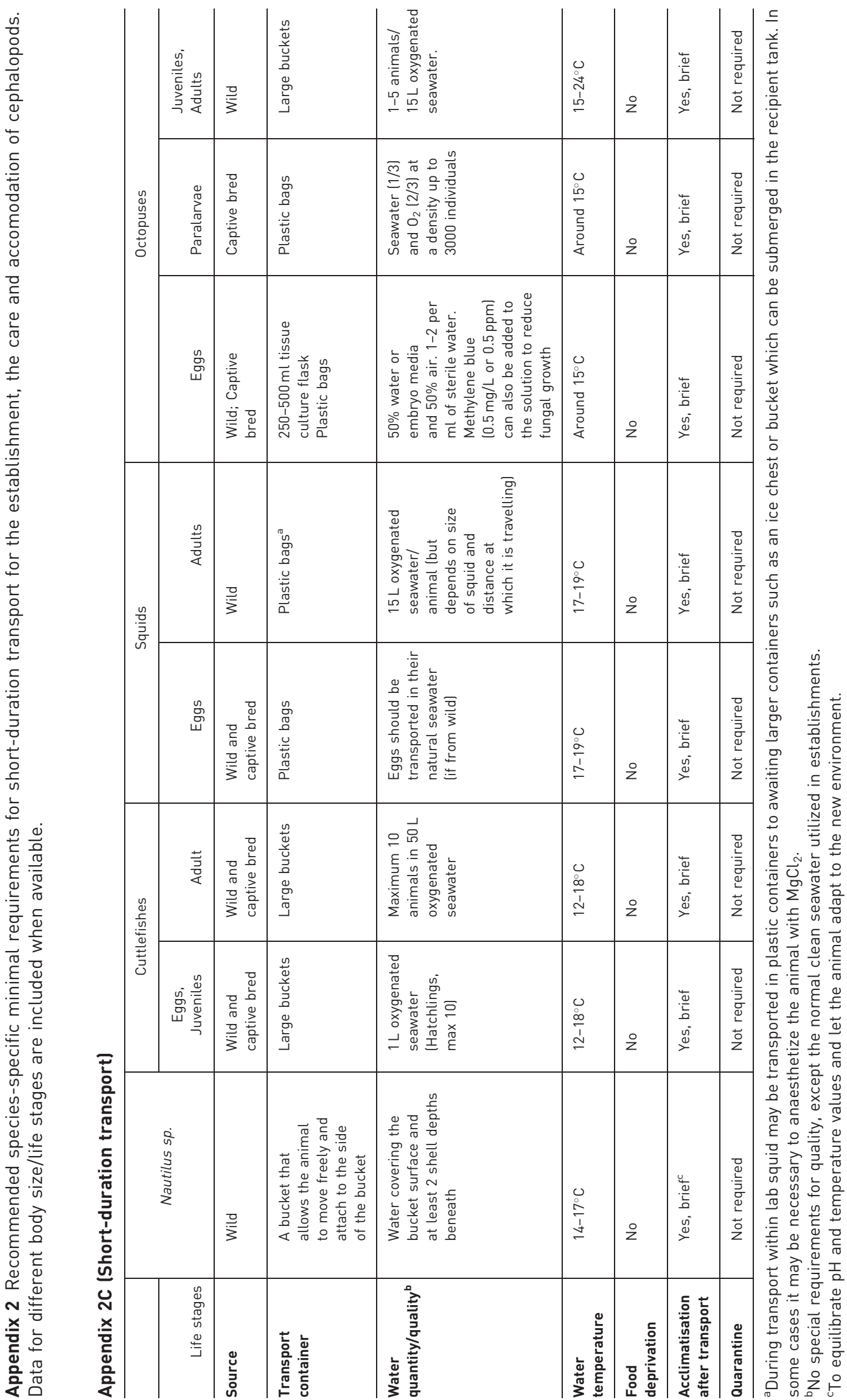



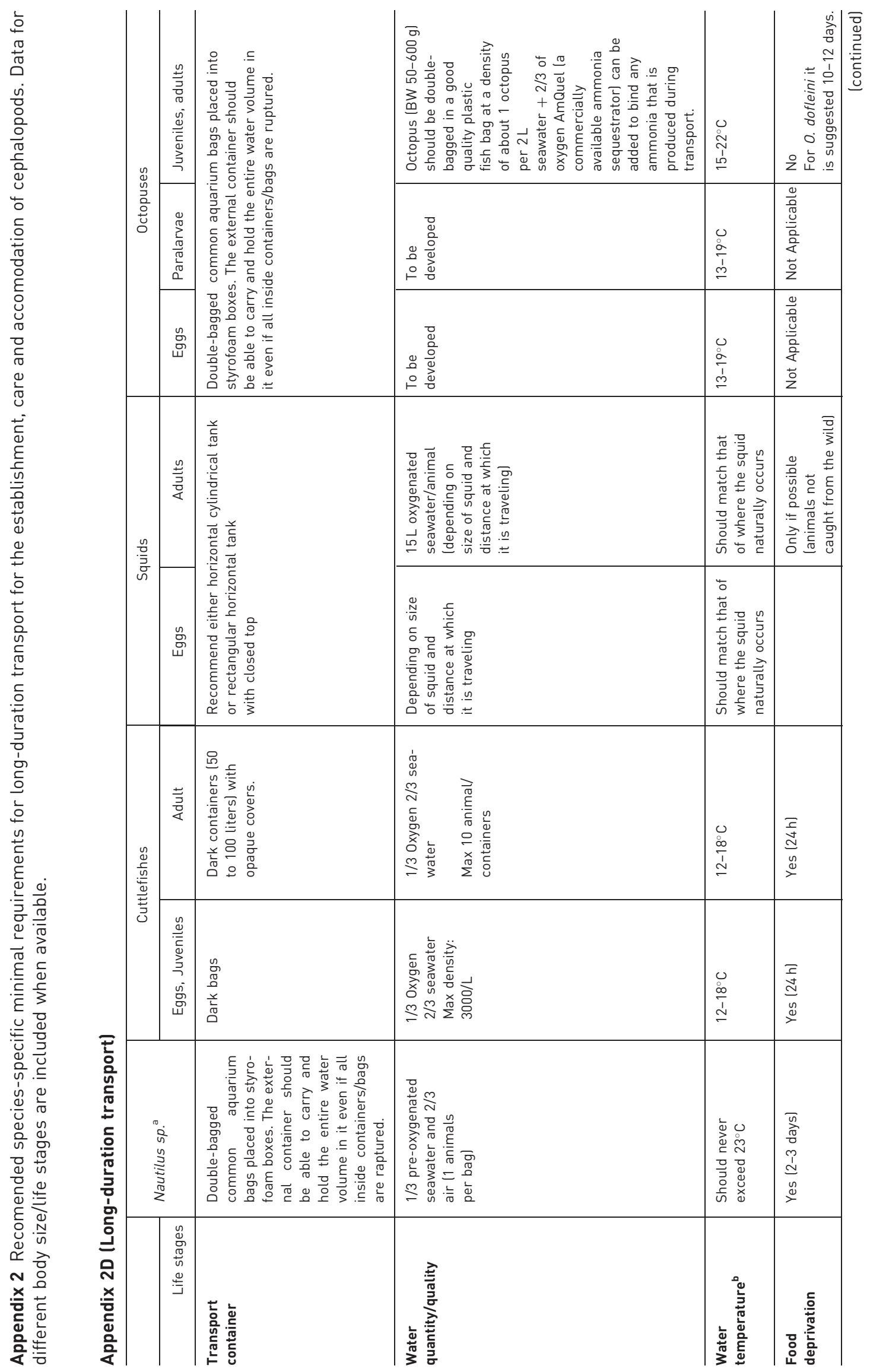


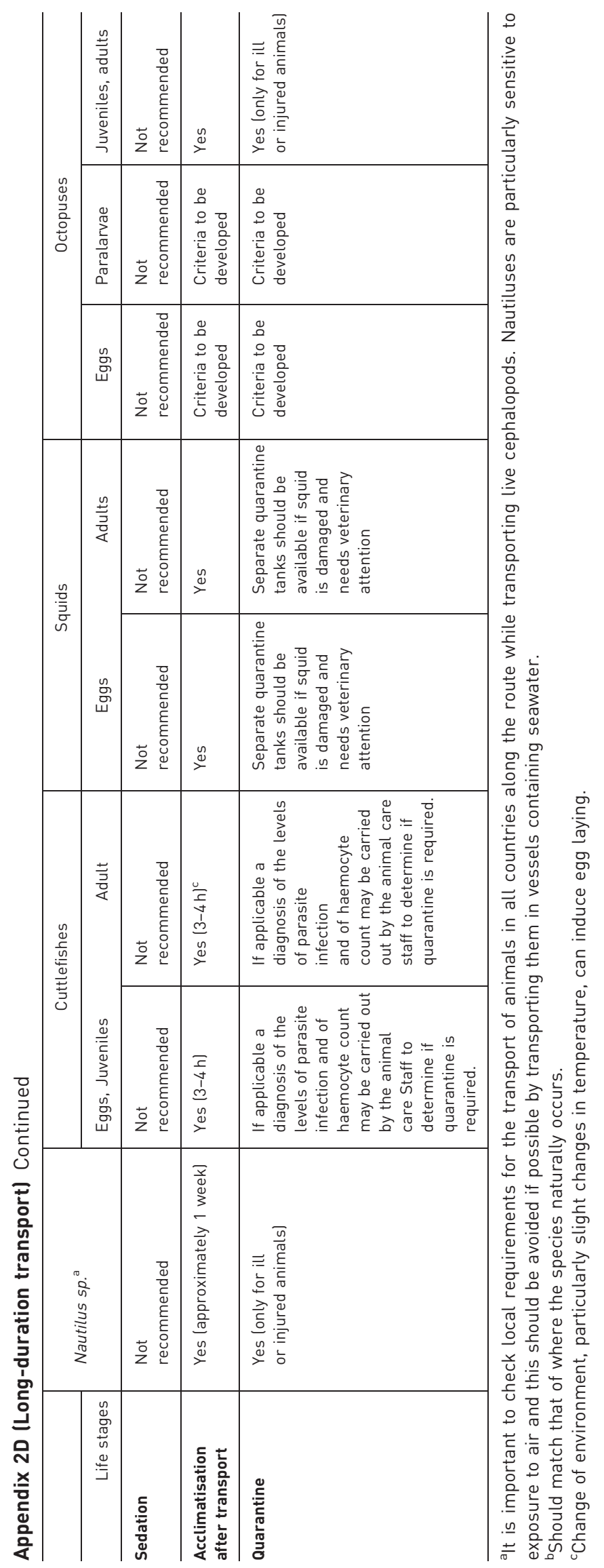




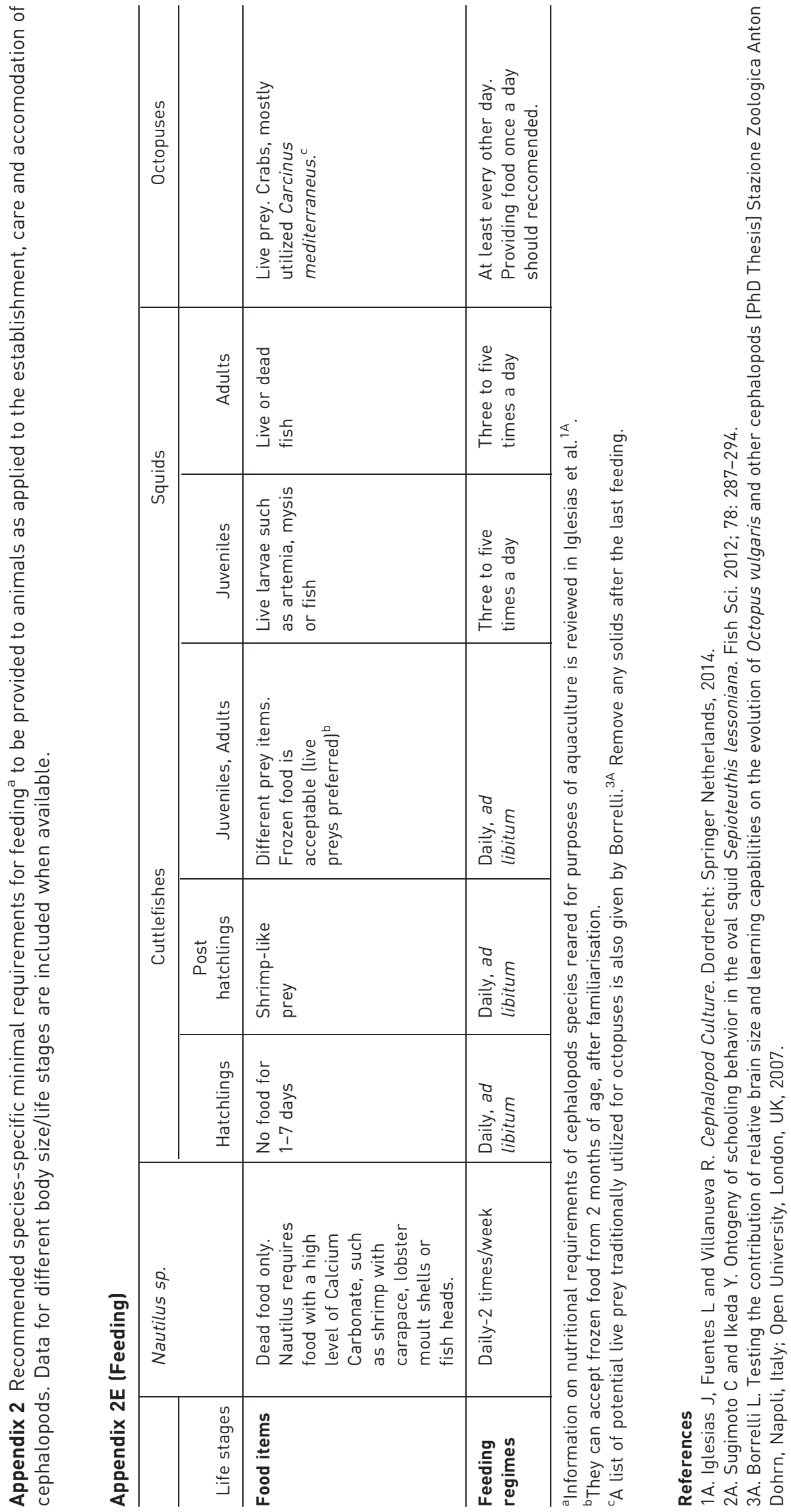


Appendix 3 - Reproductive strategies of some cephalopods species as deduced from Rocha et al. (2001) ${ }^{a}$. The list of

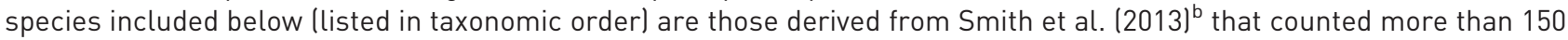
species utilised for the scientific purposes in EU over a 5-year period. The life span of cephalopods typically ranges from 6 months to 2 years; smaller tropical species tend to have shorter lives while larger, cold-water species live longer. The sole exception known to this rule is Nautilus with a life span known to be longer than 20 years.

\begin{tabular}{|c|c|c|c|c|c|}
\hline & \multicolumn{5}{|c|}{ Reproductive Strategy } \\
\hline & $\begin{array}{l}\text { Polycyclic } \\
\text { spawning }\end{array}$ & $\begin{array}{l}\text { Intermittent } \\
\text { terminal } \\
\text { spawning }^{\text {d }}\end{array}$ & $\begin{array}{l}\text { Simultaneous } \\
\text { terminal } \\
\text { spawning }^{\mathrm{e}}\end{array}$ & $\begin{array}{l}\text { Multiple } \\
\text { spawning }^{f}\end{array}$ & $\begin{array}{l}\text { Continuous } \\
\text { spawning }\end{array}$ \\
\hline Nautilus sp. & $\sqrt{ }$ & & & & \\
\hline Sepia officinalis & & V & & & \\
\hline S. elegans & & $\sqrt{ }$ & & & \\
\hline Idiosepius pygmaeus & & & & & V \\
\hline Sepiola rondeleti & & $\sqrt{ }$ & & & \\
\hline S. atlantica & & $\sqrt{ }$ & & & \\
\hline S. robusta & & $\sqrt{ }$ & & & \\
\hline Sepietta oweniana & & $\sqrt{ }$ & & & \\
\hline Rossia macrosoma & & $\sqrt{ }$ & & & \\
\hline Loligo vulgaris & & $\sqrt{ }$ & & & \\
\hline L. (Alloteuthis) subulata & & $\sqrt{ }$ & & & \\
\hline L. forbesi & & V & & & \\
\hline L. opalescens & & & V & & \\
\hline L. pealei & & V & & & \\
\hline Lolliguncula brevis & & $\sqrt{ }$ & & & \\
\hline Photololigo sp. & & $\sqrt{ }$ & & & \\
\hline Sepioteuthis sepioidea & & $\sqrt{ }$ & & & \\
\hline S. lessoniana & & $\sqrt{ }$ & & & \\
\hline Illex illecebrosus & & $\sqrt{ }$ & & & \\
\hline 1. argentinus & & $\sqrt{ }$ & & & \\
\hline I. coindetii & & $\sqrt{ }$ & & & \\
\hline Todarodes sagittatus & & V & & & \\
\hline T. angolensis & & V & & & \\
\hline T. pacificus & & & V & & \\
\hline Todaropsis eblanae & & $\sqrt{ }$ & & & \\
\hline Dosidicus gigas & & & & $V$ & \\
\hline Octopus vulgaris & & & $\sqrt{ }$ & & \\
\hline O. cyanea & & & V & & \\
\hline O. macropus & & & $\sqrt{ }$ & & \\
\hline Enteroctopus dofleini & & & $\sqrt{ }$ & & \\
\hline Eledone moschata & & & V & & \\
\hline E. cirrhosa & & & V & & \\
\hline
\end{tabular}

${ }^{a}$ For full reference to the cited document see Reference List (\#277) at the end of the paper.

${ }^{b}$ For full reference to the cited document see Reference List (\#3) at the end of the paper.

'Iteroparity legg capsules are released with a significant time interval between successive eggs spawned one by one or successive egg batches), with asynchronous ovulation and growth between egg batches.

dIteroparity, with synchronous ovulation and no growth between egg batches.

e Semelparity legg capsules are released simultaneouslyl, with synchronous ovulation and no growth between egg batches.

f Iteroparity, with group-synchronous ovulation (at least two populations of oocytes can be distinguished at some time) and growth between egg batches.

IIteroparity, with asynchronous ovulation and growth between egg batches. 